\title{
Pulmonary rehabilitation
}

Citation for published version (APA):

Augustin, I. M. L. (2022). Pulmonary rehabilitation: a learning health care model? [Doctoral Thesis, Maastricht University]. Maastricht University. https://doi.org/10.26481/dis.20220217ia

Document status and date:

Published: 01/01/2022

DOI:

10.26481/dis.20220217ia

Document Version:

Publisher's PDF, also known as Version of record

\section{Please check the document version of this publication:}

- A submitted manuscript is the version of the article upon submission and before peer-review. There can be important differences between the submitted version and the official published version of record.

People interested in the research are advised to contact the author for the final version of the publication, or visit the DOI to the publisher's website.

- The final author version and the galley proof are versions of the publication after peer review.

- The final published version features the final layout of the paper including the volume, issue and page numbers.

Link to publication

\footnotetext{
General rights rights.

- You may freely distribute the URL identifying the publication in the public portal. please follow below link for the End User Agreement:

www.umlib.nl/taverne-license

Take down policy

If you believe that this document breaches copyright please contact us at:

repository@maastrichtuniversity.nl

providing details and we will investigate your claim.
}

Copyright and moral rights for the publications made accessible in the public portal are retained by the authors and/or other copyright owners and it is a condition of accessing publications that users recognise and abide by the legal requirements associated with these

- Users may download and print one copy of any publication from the public portal for the purpose of private study or research.

- You may not further distribute the material or use it for any profit-making activity or commercial gain

If the publication is distributed under the terms of Article $25 \mathrm{fa}$ of the Dutch Copyright Act, indicated by the "Taverne" license above, 
(C) Copyright: Ingrid M.L. Augustin

Layout: Tiny Wouters

Kunstwerk omslag: Stefan Serneels (Enlightened shame)

Ontwerp omslag: Jorrit Vanderhoven

Production: ProefschriftMaken.nl

ISBN: 978-94-6423-648-4 


\title{
Pulmonary rehabilitation: a learning health care model?
}

\author{
PROEFSCHRIFT \\ ter verkrijging van de de graad van doctor aan de Universiteit Maastricht, \\ op gezag van de Rector Magnificus, Prof. dr. Pamela Habibović, \\ volgens het besluit van het College van Decanen, \\ in het openbaar te verdedigen \\ op donderdag, 17 februari 2022 om 13.00 uur
}

door

Ingrid M.L. Augustin 


\section{Promotors}

Prof. dr. E.F.M. Wouters

Prof. dr. G.G. van Merode

Prof. dr. M.A. Spruit

\section{Beoordelingscommissie}

Prof. dr. J.M.G.A. Schols (voorzitter)

Prof. dr. M.H.J. van den Beuken-van Everdingen

Prof. dr. D. Ruwaard

Prof. dr. M.J.G. Jacobs, Tilburg University

Prof. dr. K. Kenn, University of Marburg, Germany 


\section{Table of contents}

Chapter 1 General introduction - Pulmonary rehabilitation: what is in a name?

Chapter 2 Aims and outline of the thesis

Chapter 3 Understanding complexity of chronic non-communicable diseases: an integrated approach for personalized management of patients with COPD

Chapter 4 The respiratory physiome: clustering based on a comprehensive lung function assessment in patients with COPD

Chapter 5 Differential response to pulmonary rehabilitation in COPD: multidimensional profiling

Chapter 6 Comprehensive lung function assessment does not allow to infer response to pulmonary rehabilitation in patients with COPD

Chapter 7 Incorporating comprehensive assessment parameters to better characterize and plan rehabilitation for persons with COPD

Chapter 8 Multidimensional outcome assessment of pulmonary rehabilitation in traits-based clusters of COPD patients

Chapter 9 Summary and general discussion

Chapter 10 Future perspectives

Samenvatting

Impact section

List of publications

Dankwoord

Curriculum vitae 



\section{Chapter 1}

General introduction

Pulmonary rehabilitation:

what is in a name? 



\section{Definitions and goals of pulmonary rehabilitation}

The roots of pulmonary rehabilitation extend back to the time when tuberculosis was quite prevalent, before the turn of the $20^{\text {th }}$ century. ${ }^{1}$ As cited in an 1895 monograph by pulmonologist C. Denison, pulmonary invalids suffering from the residual effects of tuberculosis were offered a systematic program of exercise and breathing exercises. ${ }^{1}$ Two pioneers in the field of pulmonary rehabilitation in the mid- $20^{\text {th }}$ century were $A$. Barach and A. Haas. ${ }^{1}$ Barach was the first to apply positive pressure mechanical ventilation in the 1940s and the first to administer oxygen during exercise in the $1950 \mathrm{~s}^{2}$ A. Haas recognized that physical activity was associated with weight gain and feelings of well-being when he administered his therapy for tuberculosis. ${ }^{1}$

Since the 1970s, interest in pulmonary rehabilitation as an art and method of systematized, multidisciplinary care for patients with chronic respiratory conditions has increased progressively, and a more scientific basis for pulmonary rehabilitation has been established based on well-designed trials with valid, responsive and interpretable outcome measures. Accordingly, the formulation of definitions has changed, but all definitions have in common the improvement of the health of the patient and the attenuation of the burden of the disease.

The first authoritative statement on pulmonary rehabilitation from the American College of Chest Physicians and the American Thoracic Society in 1974 introduced pulmonary rehabilitation as "an art of medical practice wherein an individually tailored, multidisciplinary program is formulated which, through accurate diagnosis, therapy, emotional support and education, stabilizes or reverses both the physio-and psychopathology of pulmonary diseases and attempts to return the patient to the highest possible capacity allowed by his pulmonary handicap and overall life situation". ${ }^{3}$

Subsequently, several statements and guidelines have been established, and definitions have been reworded. Besides the development in the field of pulmonary rehabilitation, these consecutive definitions reflect the integration of the outcomes and goals of chronic care in general.

In 1992, the European Respiratory Society ${ }^{4}$ reformulated pulmonary rehabilitation as "an intervention that aims to restore patients to an independent, productive and satisfying life and prevent further clinical deterioration to the maximum extent compatible with the stage of the disease".

In 1994, the National Institutes of Health" defined pulmonary rehabilitation as "a multidimensional continuum of services directed to persons with pulmonary disease and the involvement of their families, usually by an interdisciplinary team of specialists, with 
the goal of achieving and maintaining the individual's maximum level of independence and functioning in the community".

In 1997, the European Respiratory Society ${ }^{6}$ introduced pulmonary rehabilitation as "a process which systematically uses scientifically based diagnostic management and evaluation options to achieve the optimal daily functioning and health-related quality of life of individual patients suffering from impairment and disability due to chronic respiratory diseases". In the same year, the British Thoracic Society ${ }^{7}$, in its guidelines for the management of COPD, defined pulmonary rehabilitation as "a restoration of the best possible physiological, psychological and social potentials for the individual", quoting the American Thoracic Society definition of 1981.

In 1999, the American Thoracic Society reformulated pulmonary rehabilitation as a multidisciplinary program of care for patients with chronic respiratory impairment that is individually tailored and designed to optimize physical and social performance and autonomy. ${ }^{8}$

According to an official American Thoracic Society/European Respiratory Society statement (2006), pulmonary rehabilitation was defined as "an evidence-based, multidisciplinary, and comprehensive intervention for patients with chronic respiratory diseases who are symptomatic and often have decreased daily life activities. Integrated into the individualized treatment of the patient, pulmonary rehabilitation is designed to reduce symptoms, optimize functional status, increase participation, and reduce health care costs through stabilizing or reversing systemic manifestations of the disease" ${ }^{\prime 9}$

The latest definition was adopted by the European Respiratory Society and American Thoracic Society in 2013 ${ }^{10}$ : "Pulmonary rehabilitation is a comprehensive intervention based on a thorough patient assessment followed by patient-tailored therapies, which include, but are not limited to, exercise training, education, and behavior change, designed to improve the physical and psychological condition of people with chronic respiratory disease and to promote the long-term adherence of health-enhancing behaviors".

The commonality among these definitions of pulmonary rehabilitation are the focus on chronic respiratory patients and their caregivers, the individualization of the program and the multidimensionality and comprehensiveness of the intervention. They all have in common the ability of the patient to adapt and manage one's own well-being. From the beginning, pulmonary rehabilitation shifted the emphasis to resilience and well-being, basic components of the current concept of positive health. ${ }^{11}$ In fact, the definitions of pulmonary rehabilitation evolved with the concepts of management and care of chronic conditions in the previous decades. ${ }^{12-14}$ Supplemental Table S1.1 summarizes the pulmonary rehabilitation goals of the different definitions, classified into the dimensions 
of physical, mental and social functioning of health as recently defined by the World Health Organization. ${ }^{11}$

Although priority may differ, from the onset, it has been recognized by clinicians that the patient's family should also be involved in the pulmonary rehabilitation program. In 2013, the goal of pulmonary rehabilitation was even extended to include behavioral change and the promotion of long-term adherence to health-enhancing behaviors. Dimensions such as social participation and options for making better use of patient's abilities to cope, adapt and self-manage were intrinsically part of the pulmonary rehabilitation approach.

While pulmonary rehabilitation includes a broad spectrum of interventions that aim to improve physical, psychological and social functioning ${ }^{10}$, pulmonary rehabilitation teams are assumed to have the skills to diagnose, manage and understand the needs of patients and caregivers. ${ }^{10}$ In contrast to the traditional point of view in which needs in different domains are addressed by specialists from those specific domains, pulmonary rehabilitation teams consist of physicians and other health care professionals such as physiotherapists, respiratory therapists, nurses, psychologists, behavioral specialists, exercise physiologists, nutritionists, occupational therapists and social workers. Despite the common goals included in statements and definitions, large differences are found in the content and organizational aspects of pulmonary rehabilitation programs worldwide $^{15}$, and simple exercise and education interventions are even considered as comprehensive pulmonary rehabilitation. ${ }^{16,17}$ Furthermore, over the years, pulmonary rehabilitation programs have narrowed their outcomes to the improvement of health status, exercise performance and the attenuation of symptoms as their main goals. ${ }^{15}$ These developments stand in sharp contrast to the goals of pulmonary rehabilitation as formulated in all definitions. As a consequence, important components of pulmonary rehabilitation in achieving improvement in the global dimension of health remain unaddressed. In the absence of process and performance metrics, it remains difficult at present to compare pulmonary rehabilitation programs and to enable benchmarking between programs and settings.

In recent decades, pulmonary rehabilitation as a field of respiratory medicine has been confronted with important contrasting developments: on the one hand, enabling the individual to achieve the best possible health, and on the other hand, applying evidencebased knowledge and methods to intervene in all these dimensions of health. These developments will be addressed in the following section. 


\section{COPD: from airflow limitation towards a complex, chronic syndrome}

All definitions of pulmonary rehabilitation are targeted to patients with chronic respiratory conditions. At present, most pulmonary rehabilitation programs address the needs of patients with chronic obstructive pulmonary disease (COPD). As recently as 2001, the Global Strategy for the Diagnosis, Management and Prevention of COPD (GOLD) defined COPD as a disease state characterized by airflow limitation that is not fully reversible. This airflow limitation is usually both progressive and associated with an abnormal inflammatory response of the lungs to noxious particles and gases. ${ }^{18}$ Two decades later, GOLD defined COPD as a common, preventable and treatable disease that is characterized by persistent respiratory symptoms and airflow limitation due to airway and/or alveolar abnormalities usually caused by significant exposure to noxious particles and gases and influenced by host factors including abnormal lung development. Significant comorbidities may have an impact on morbidity and mortality. ${ }^{19}$ Although GOLD recognizes COPD as a complex condition with many different components and mechanisms contributing to its pathophysiology and clinical presentation, daily management is still based on airflow limitation, limitations or symptoms and exacerbation risk. Additionally, GOLD recognizes the heterogeneity of COPD patients and the need for flexibility in tackling individual characteristics and meeting the needs of each patient. ${ }^{20}$

Agusti and MacNee ${ }^{21}$ suggested using a "control panel" that includes at least three dimensions of disease (severity, activity and impact) to visualize the complexity of COPD in a single patient at a given point in time in order to move COPD management towards personalized medicine. These three dimensions of biological and clinical variables can provide complementary and relevant information for the proper management of the individual patient. It can be customized to the needs of the patient and the available resources (e.g., rural versus urban health care centers and primary versus specialized care). ${ }^{22}$ The "control panel" may further serve as a clinical decision support system. ${ }^{23}$ However, exactly which treatable clinical characteristics should be assessed and which methods and cut-off points should be used has not yet been formally validated.

Over the last few decades, it has been increasingly recognized that, in addition to the heterogeneous involvement of the respiratory impairment, the presence of other chronic conditions such as cardiovascular diseases, depression, osteoporosis, anemia and diabetes substantially contributes to the severity of the disease. ${ }^{24}$ Comorbidities not only affect symptom burden, functional performance and health status in patients with COPD, but also the risk of hospitalization and mortality. ${ }^{25-27}$ In COPD patients admitted for pulmonary rehabilitation to our own center, at least five comorbidity clusters could be identified: one with less comorbidity, and cardiovascular, metabolic, cachectic and psychological clusters. These comorbidity clusters differed significantly in health status: 
in particular, the psychological cluster, characterized by anxiety and depression, reported more dyspnea and poorer health status. ${ }^{28}$ However, these comorbidities do not prevent COPD patients from improving exercise capacity and health status after pulmonary rehabilitation. ${ }^{29}$ Again, this diagnosis and the management of COPD beyond the lungs warrant an individualized approach as part of an integrated disease management plan. ${ }^{30}$

Recently, a "label-free, precision medicine approach based on the concept of 'treatable traits' has been introduced to the diagnosis and management of chronic airway diseases" 31 in order to offer a precision medicine intervention, defined as "treatments targeted to the needs of individual patients on the basis of genetic, biomarker, phenotypic, or psychosocial characteristics that distinguish a given patient from other patients with similar clinical presentations". ${ }^{32}$ A broad set of pulmonary and extrapulmonary treatable traits as well treatable behavior and lifestyle traits has been summarized, reflecting the variability in clinical manifestations of chronic respiratory diseases. Indeed, COPD has been extensively reported as a complex disease affecting patients' health beyond the lungs, with a variety of intra- and extra-pulmonary components and considerable variability between individuals. ${ }^{28,33-37}$ However, data about the outcomes of this kind of integrated treatable traits strategy are still lacking.

Given the increasing understanding of COPD as a heterogeneous and complex disease, it is important to accentuate that in this context, "complex" means that COPD has a number of intra- and extra-pulmonary components whose dynamic interactions over time are not linear, whereas "heterogeneous" indicates that not all of these components are present in all individuals at any given point in time. ${ }^{38}$

Current COPD recommendations and classifications poorly address this complexity and heterogeneity. ${ }^{39,40}$ Therefore, the characteristics of individuals with COPD referred for pulmonary rehabilitation vary greatly. As a consequence, the frequency and number of treatable traits in patients referred for pulmonary rehabilitation make this form of personalized management a real challenge.

\section{Pulmonary rehabilitation: current challenges}

\section{A thorough assessment?}

Referring to the latest definition ${ }^{10}$, pulmonary rehabilitation as a comprehensive intervention implies a thorough assessment followed by a patient-tailored program. Statements and guidelines ${ }^{4-10,41-43}$ endorse the importance of an initial patient assessment which includes a full clinical, physiological, psychological and social evaluation to determine, on an individual basis, the content and composition of the 
pulmonary rehabilitation program. As described in Supplemental Table S1.2, an initial assessment is recommended in all guidelines and statements, but none of them clearly formulates the full content of these pre-rehabilitation measurements. While in $1999^{8}$ the measurement of dyspnea, exercise ability, health status and physical activity was proposed, in $2006^{9}$ a pre-assessment on psychological and social considerations and an evaluation on common feelings was recommended. The 2013 ATS/ERS statement ${ }^{10}$ recommends evaluation of exercise performance in order to individualize exercise prescription. It was stated that a maximal cardiopulmonary exercise test is considered not only a prerequisite for defining the factors that contribute to exercise limitation and identifying a suitable exercise prescription, but also for helping to ensure the safety of the intervention. At that time, a physiological rationale supporting the effectiveness of pulmonary rehabilitation was considered very important, and various landmark studies demonstrated that even in patients with severe COPD, rigorous exercise training resulted in substantial improvements in exercise tolerance and physiological training effects. ${ }^{44,45}$ The British Thoracic Society considers an initial assessment to be an opportunity when it comes to assessment and referral for treatment of comorbidities. ${ }^{43}$ The unspecified description of the content of the assessment program in order to offer an integrated, individualized program is in sharp contrast to the description of treatable traits as an avenue to precision medicine in COPD. ${ }^{31}$

In addition, a lack of consensus on the content of pulmonary rehabilitation programs also contributes to imprecise criteria for referral. The effectiveness of a pulmonary rehabilitation program covers a wide spectrum of variables such as medical resource consumption and cost-effectiveness as well as improvements in respiratory symptoms, exercise ability, quality of life, activities of daily living, psychological well-being, cognitive functioning, nutrition, body composition and health behavior. GOLD prescribes pulmonary rehabilitation for all patients with relevant symptoms and/or a high risk for exacerbations and recommends a program that includes patient goals. Such a program has to be designed and delivered in a structured manner, taking into account the individual's COPD characteristics and comorbidities. ${ }^{19}$ GOLD still insists on the role of spirometry in the stratification of the burden of the disease, but this measurement does not determine the impact on the patient's health status and the risk of future events (exacerbations, hospital admissions or death). ${ }^{19}$ It has been well known for decades that physiological measures of disease severity such as $\mathrm{FEV}_{1}$ poorly predict the burden of disease. ${ }^{46}$ Nevertheless, despite $\mathrm{FEV}_{1}$ 's limitations, $\mathrm{FEV}_{1}<50 \%$ of predicted is still used in clinical practice as a referral criterion for pulmonary rehabilitation. ${ }^{47}$

It seems that current rehabilitation practice still largely reflects a disease-centered framework with a physiologic rationale that is focused on exercise performance and poorly addresses what matters most to these patients despite a variety of statements and guidelines. This clearly highlights the need for more patient goals-directed care. ${ }^{48}$ 


\section{Pulmonary rehabilitation and evidence-based medicine}

Since the '80s of the previous century to the most recent statements, the respiratory community has embraced pulmonary rehabilitation as a comprehensive, broadspectrum intervention. Indeed, to achieve the personal health outcomes that patients hope to obtain, pulmonary rehabilitation programs put in place a wide spectrum of interventions: different forms of exercise training programs, psychosocial and behavioral interventions, nutritional therapy, oxygen therapy, advance care planning and noninvasive mechanical ventilation, as well as education programs for patient and family. Pulmonary rehabilitation programs address the multiple needs of patients with a chronic lung disease but are only described as sets of tools and disciplines (see Supplemental Tables S1.3 and S1.4). The focus on outcomes may differ over time, but all these treatment dimensions are consecutively described and positioned in different statements. To illustrate, while the National Institutes of Health ${ }^{5}$ at that time gave an extended overview of interventions influencing psychological variables, anxiety and depression are only described as an outcome and an unexplored area in the latest American Thoracic Society/European Respiratory Society statement. ${ }^{10}$ In 2013, The American Thoracic Society/European Respiratory Society recognized the complex nature of COPD, its multisystem manifestations and frequent comorbidities with treating comorbidities as part of the pulmonary rehabilitation program. As a consequence, it has been recommended that pulmonary rehabilitation should take this disease complexity into account. In practice, guidelines are lacking to systematically address this complexity, define its dimensions and allocate the right patient to the right pulmonary rehabilitation setting. ${ }^{15,49}$ Over the years, pulmonary rehabilitation has been built on a combination of expert opinions, tacit knowledge, the experiences and skills of a wide variety of health care providers, theoretical reasoning from basic sciences and the needs and wishes of patients.

The paradigm shift of evidence-based medicine (EBM) also influenced the content of pulmonary rehabilitation. Evidence-based medicine was introduced in 1996 and was defined as the conscientious, explicit and judicious use of current best evidence in making decisions about the care of individual patients. ${ }^{50}$ EBM committed to making clinical practice more scientifically and empirically grounded, thereby achieving safer, more consistent and more effective care. ${ }^{50}$ It was argued that EBM, if practiced knowledgably and compassionately, could accommodate basic scientific principles, the subtleties of clinical judgment and the patient's clinical and personal idiosyncrasies. ${ }^{51}$ Evidence-based became a quality mark, and randomized controlled trials (RCTs) and meta-analyses are still considered the major tools of EBM. As a consequence, developments and innovation have been reduced to evidence-based principles. Evidence from clinical trials is summarized in so-called Cochrane reviews. ${ }^{52}$ In 1996, Lacasse et al carried out a first meta-analysis of RCTs of pulmonary rehabilitation in patients with COPD based on outcomes of functional or maximal exercise capacity, health-related 
quality of life (HRQL) or both. Pulmonary rehabilitation was defined as exercise training with or without education, psychological support or both. ${ }^{53}$ They strongly supported pulmonary rehabilitation with exercise training as part of the management plan for patients with COPD and concluded that pulmonary rehabilitation led to greater improvements in HRQL and functional exercise capacity compared to usual care. ${ }^{53}$ The authors highlighted the need for evidence-based overviews of the literature as a basis for implementing new rehabilitation programs. ${ }^{53}$ Almost 20 years later, McCarthy et al, in the latest Cochrane review ${ }^{54}$, included the same selection criteria and selected RCTs of pulmonary rehabilitation in patients with COPD in which HRQL and/or functional or maximal exercise capacity were measured: pulmonary rehabilitation was defined as exercise training with or without education or any form of additional intervention. ${ }^{54}$ The conclusions of this meta-analysis were in agreement with previous meta-analyses ${ }^{53,55,56}$ : pulmonary rehabilitation relieves dyspnea and fatigue, improves emotional function and enhances the sense of control that individuals have over their condition. The Cochrane Airways editorial even made the unusual decision to close the review of the evidence of pulmonary rehabilitation with this latest update to the Cochrane Review of McCarthy et al.. ${ }^{54}$ The Cochrane Airways editors have recognized the limitations of RCTs as interventions cannot be blinded. The editorial board concluded that the clinical research conducted delivered meaningful outcomes, and that those who apply the intervention, those who receive it and those who fund it can act with confidence. ${ }^{57}$ Obviously, outcome measures included in this Cochrane Review were limited to evaluating exercise performance and HRQL.

What is striking is both the narrow definition of pulmonary rehabilitation and the shift from a global dimension of health towards fragmented disease-related outcomes. Instead of a comprehensive intervention, pulmonary rehabilitation was defined in these meta-analyses as an intervention that includes exercise training, with or without education or any form of additional intervention. ${ }^{54}$ Furthermore, outcome measures chosen for establishing efficacy are largely limited to symptom reduction as originally proposed by GOLD: reduction in exercise limitation and dyspnea and improvement in $\mathrm{HRQL}^{58}$, ignoring the more fundamental goals of pulmonary rehabilitation. Furthermore, the average results of the findings of these RCTs have been generalized to decisions about real-life patients: the multidimensional and differential impairment in these highneed patients defy these evidence-based efforts in pulmonary rehabilitation. ${ }^{5}$ However, over the years, the language of EBM contributed to a creeping managerialism and politicization not only for pulmonary rehabilitation, but for clinical practice in general. ${ }^{59}$ These developments ignore the conclusions of Sackett et al that the information of any guideline or review must be integrated with individual clinical expertise in deciding whether and how it matches the patient's clinical state, predicament and preferences, and thus whether it should be applied: only in that way does EBM prove to be knowledgeable and compassionate. ${ }^{50}$ Particularly for pulmonary rehabilitation as an individualized intervention, a "real" EBM approach must be advocated-one which is 
built on real, shared decision-making that matters to the patient and on strong human and ethical care aspects. ${ }^{60}$

\section{From global dimension of health towards fragmented disease-related outcomes}

In the first authoritative statement on pulmonary rehabilitation, the final goal of intervention, defined as an art of medical practice, was to bring the patient suffering from a pulmonary disease condition to the highest possible capacity allowed by his pulmonary handicap and overall life situation. ${ }^{3}$ Weatherall argued that this art of medicine describes a holistic approach to the care of patients ${ }^{61}$ : art of medicine not only includes skills in diagnosis and treatment, it also encompasses the management of every aspect of patients' reactions to their illness and its impact on their lives. ${ }^{61}$ Consistent with this approach, in the eighties of the previous century (see Table S1.3), outcomes of pulmonary rehabilitation were directed towards the patient feeling better not only in terms of less dyspnea, but also having greater confidence, less depression, anxiety and panic and less frequent insomnia. Furthermore, greater activity, increased endurance and strength, greater range of function, self-control and self-management and more effective visits to the physician had to be achieved after pulmonary rehabilitation. ${ }^{62}$ The National Institutes of Health challenged this concept of pulmonary rehabilitation as an "art," arguing that this approach did not lend itself to meaningful discussions about opportunities for research. ${ }^{5}$ The overview in Table S1.1 clearly demonstrates that the domain of mental functioning is not always clearly described as a goal of pulmonary rehabilitation..$^{5,6,8,9,41-43}$ On the other hand, pulmonary rehabilitation goals did not always correspond with recommended outcome measures. As an example, as a result of a National Institutes of Health discussion during their workshop in $1994^{5}$ concerning all domains, they finally included in their definition "... achieving and maintaining the individual's maximum level of independence and functioning in the community."

This art of medicine was later described as the dark period of pulmonary rehabilitation and was rejected in favor of clinical judgement based on rational thinking or EBM. ${ }^{63-65}$ Indeed, driven by a growing skepticism towards the lack of established rationale concerning pulmonary rehabilitation, the original patient goals-directed care moved into a more disease-centered approach, even for patients with multiple underlying conditions. In the absence of relevant reversibility of the underlying pathology in patients with COPD, disease-centered outcomes have been reduced to attenuation of symptoms, in particular dyspnea and exercise intolerance, and to the reduction of risks of exacerbations. Furthermore, the focus on exercise conditioning as an essential component of pulmonary rehabilitation is also the result of the drive within the rehabilitative community to tackle the prevailing doubts concerning the value of rehabilitative exercise. The argument was that the exercise tolerance of patients with COPD is limited by their lungs, and it was pointed out that exercise conditioning does not improve lung function. Furthermore, it was doubted that patients with COPD could 
exercise at an intensity sufficient enough to exceed their critical training threshold above which improvements in muscle function could be expected. It was assumed that improvements in exercise tolerance after pulmonary rehabilitation could be ascribed to psychological factors including improved motivation and decreased sensitivity to dyspnea. ${ }^{66}$ In 1995, Ries et al showed clearly that rehabilitation programs that includes exercise training resulted in significantly larger improvements in exercise capacity, symptoms and quality of life than educational programs alone. ${ }^{67}$ This study provided an answer as to whether exercise training was effective. Later on, attention shifted towards skeletal muscle dysfunction in these patients. ${ }^{68}$

Building on this strong physiologic rationale to support effectiveness, guidelines and statements ${ }^{6-10,41,42}$ focus on exercise tolerance, dyspnea and health-related quality of life as outcome measures of pulmonary rehabilitation. Intriguingly, similar outcomes were generally applied to evaluate pharmacological therapy in COPD patients. It was argued that pulmonary rehabilitation improved patients to a greater degree than any other therapy.

Furthermore, upon analysis of these various Cochrane Reviews and intervention studies in COPD in general, it appears that quality of life, HRQL, functional status and the evaluation of symptoms are often used interchangeably ${ }^{69,70}$, and as a consequence, this leads to confusion and inappropriate use of these terms. ${ }^{71}$ In Figure 1.1, the relation between health status, functional status, quality of life and health-related quality of life is illustrated. ${ }^{69,72}$ The quality of a person's life is a holistic, self-determined evaluation of satisfaction, with issues important to the person. ${ }^{69}$ Quality of life is influenced by numerous factors, including financial status, housing, employment, spirituality, social support network and health. ${ }^{69}$ A more restrictive measurement of quality of life is HRQL, or the impact of a disease on quality of life. ${ }^{69}$ The term "functional status" is used to describe a person's ability to function within the physical, social and emotional domains. ${ }^{69}$ As shown in Figure 1.1, health status encompasses HRQL as well as functional status. In clinical practice and research, it seems that quality of life is indiscriminately used as an umbrella term, overemphasizing the impact of disease-related health status. $^{71}$ 


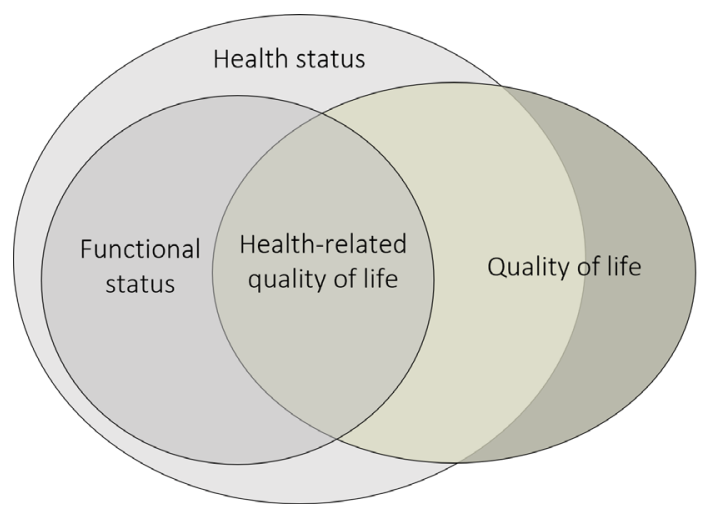

Figure 1.1 Relation between health status, functional status, quality of life and health-related quality of life. This Figure is adapted with permission of the American Thoracic Society, copyright (C) 2021 American Thoracic Society. ${ }^{69}$

Patient-related outcomes (PROs) which reveal the trajectory of symptoms or perceived health and the effect of interventions in clinical practice as well as research, have gained increasing importance. ${ }^{72}$ PRO is "any report of the status of a patient's health condition that comes directly from the patient, without interpretation of the patient's response by a clinician or anyone else". ${ }^{73}$ Given the limitations of measuring HRQL or health status as outlined above, the use of PROs in order to evaluate and compare interventions continues to be a challenge. Furthermore, these PROs must be personalized: selfmanagement abilities and health status still depend on emotional intelligence (i.e., the capacity to understand and manage personal thoughts and feelings) and are positively influenced by interpersonal communication and social well-being. ${ }^{74}$ A qualitative study revealed that patients struggle to accept their disease, which then even precludes patients from making progress during their pulmonary rehabilitation program. ${ }^{75}$ Moving towards patient goals-directed care also infers that the patient will need to be involved in the choice of care options within the context of the patient's desired outcomes and care preferences. Such an approach not only implies a definition of goals but also the acceptance of workloads by the patient. ${ }^{48}$ These developments frequently assume fundamental behavior change on that part of the patient as well as an acceptance of the diagnosis and their limitations, a realistic disease understanding and a rational response to the disease in order to enable control and self-management. ${ }^{76}$ Behavior change is incorporated in the latest definition of pulmonary rehabilitation ${ }^{10}$, but it will be important to analyze and focus on key determinants of health behaviors in order to develop more effective interventions to change those behaviors and link those changes to the overall goals of patient value-based care. ${ }^{77}$ 


\section{From individualized to "one size fits all"}

Exercise training and education are considered the cornerstone of every pulmonary rehabilitation program. Developments in current care delivery over the last few decades are described in this section.

\section{Exercise training}

Exercise therapy is a challenge in patients with limited reserves. For these patients, individualized programs which take into account the patient's capacities in order to achieve physiologic benefits ${ }^{17,45}$ are required. In general, an exercise program that consists of high-intensity endurance and resistance training is advised for patients with COPD. ${ }^{78}$ However, depending on the patient's limitations and the symptoms experienced, alternative exercise modalities are available to ascertain an optimal, individualized training format: interval training, neuromuscular electrical stimulation, whole body vibration, single-leg exercise training, eccentric training, training with oxygen supplements, training with helium-oxygen breathing, training with non-invasive ventilation support and inspiratory muscle training. ${ }^{10,17}$ All these interventions have proven effective in RCTs. The implementation of these interventions in patients with severe as well as moderate COPD is based on expert judgment by the pulmonary rehabilitation team and requires supervision as well as a strong relationship with the patient to achieve and maintain training effects. ${ }^{45,78,79}$ Clearly, even the type and intensity of exercise prescription needs to be individualized. ${ }^{78}$

Merely defining exercise training as a component of pulmonary rehabilitation leaves room for a scattering of different interpretations as well as modalities to deliver exercise programs. Even unsupervised home-based training interventions currently qualify as pulmonary rehabilitation programs. Furthermore, by reducing outcomes to a 6-minute walk distance, RCTs claim that this form of training is equivalent to center-based pulmonary rehabilitation, again with supervised group training as a stand-alone intervention. ${ }^{80}$ This blind drive towards the best evidence neglects completely the complexity of underlying problems and is misleading in its choice of simple outcome measures to establish efficacy. ${ }^{60}$ The uncritical over-rating of interventions such as pulmonary rehabilitation has contributed to tremendous confusion concerning the content and organization of pulmonary rehabilitation programs. Wuytack et al acknowledged this incorrect use of the definition of pulmonary rehabilitation in their systematic review and meta-analysis of exercise training programs, regardless of the inclusion of additional pulmonary rehabilitation components. ${ }^{81}$ In this review and metaanalysis that includes 10 studies, there was low to moderate evidence that outpatient and home-based exercise training programs are equally effective. ${ }^{81}$ Although they indicated that it is likely that the beneficial effects of exercise training programs as identified in the McCarthy et al. review ${ }^{54}$ can be obtained across settings and that different settings probably result in little to no difference in HRQL and exercise 
capacity $^{81}$, they emphasized the need for health services that could tailor the setting of programs to best suit the local context, health services resources and, importantly, patients' needs while taking into consideration patient safety, particularly in unsupervised settings. ${ }^{81}$

New initiatives to improve access to pulmonary rehabilitation programs continue to arise, from comprehensive interventions performed by teams consisting of multiple specialists to mono-dimensional interventions such as exercise training or a behavioral and community-based exercise intervention called "Urban Training". Although these studies are testing the effectiveness of alternative models in order to improve access to pulmonary rehabilitation, it remains debatable whether those alternative pulmonary rehabilitation programs may be considered a substitute for traditional programs. ${ }^{82} \mathrm{~A}$ greater emphasis should be made on allocating the right patient to the right type of exercise-related care at the right moment ${ }^{83}$ instead of the current "one size fits all" approach that only includes RCT outcomes.

Recently, a patient profiling model of exercise-based care for patients with COPD was described (see Figure 1.2) which includes delineation of those patients who are candidates for secondary or tertiary pulmonary rehabilitation programs based on systematic quantification of the disease burden experienced by the individual patient. ${ }^{83}$ Such models can be very helpful not only in revisiting the values of pulmonary rehabilitation, but also in genuinely addressing the varying impact of the disease on the adaptability and well-being of the individual patient. 


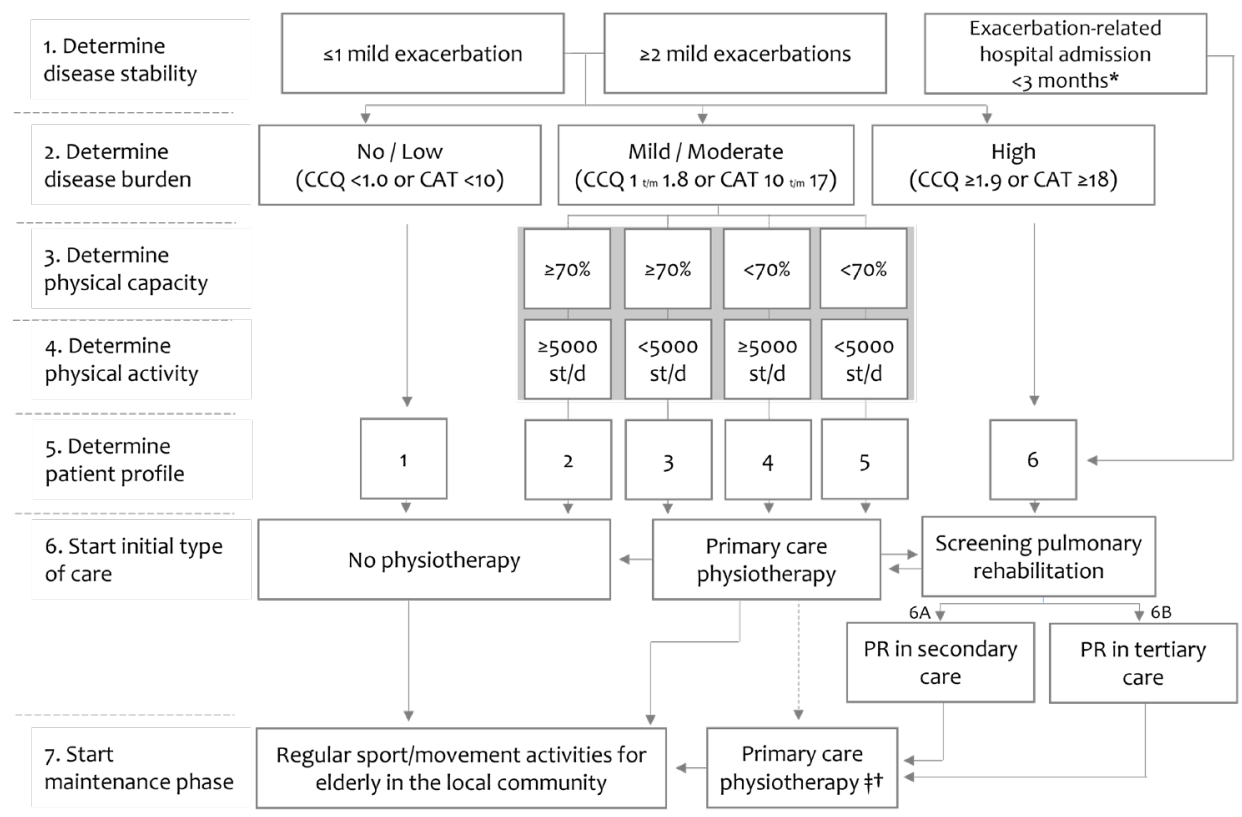

Figure 1.2 Flowchart for exercise-based care for patients with COPD. This Figure was published in Sports Medicine, Volume 50, Martijn A. Spruit et al. Profiling of Patients with COPD for Adequate Referral to Exercise-Based Care: The Dutch Model. Pages 1421-1429 (2020). ${ }^{83}$

\section{Patient education}

Patient education is the process of teaching of and learning by patients in all clinical settings in a planned, systematic, sequential and logical manner to manage the health needs they experience and increase the patient's competence in managing his or her own health requirements. ${ }^{84}$ As illustrated in Table S1.3, patient education, that includes the enhancement of knowledge and skills of family members is generally considered an important component of a pulmonary rehabilitation program: exercise programs combined with patient education are frequently defined as a comprehensive, individualized and evidence-based pulmonary rehabilitation program. ${ }^{15,40}$ As with exercise training, education interventions are clearly specified in guidelines and statements (see Table S1.3), yet these remain rather generic, leading again to divergent approaches in clinical practice. Stoilkova et al systematically evaluated 67 interventions which incorporated education for patients with COPD and found a heterogeneous and wide variation in the content and method of delivery of these educational interventions. ${ }^{85}$ Figure 1.3 provides an overview of the educational topics adopted from practice guidelines and pulmonary rehabilitation statements, with the percentage of studies included in each education topic. ${ }^{85}$ The authors emphasized the need for alignment between the educational topics incorporated into the existing programs and 
those recommended by the COPD guidelines, and the involvement of various professionals and the combined use of methods. ${ }^{85}$ Indeed, printed material and/or brochures as well as demonstrations and practice were the predominant tools and methods. ${ }^{85}$

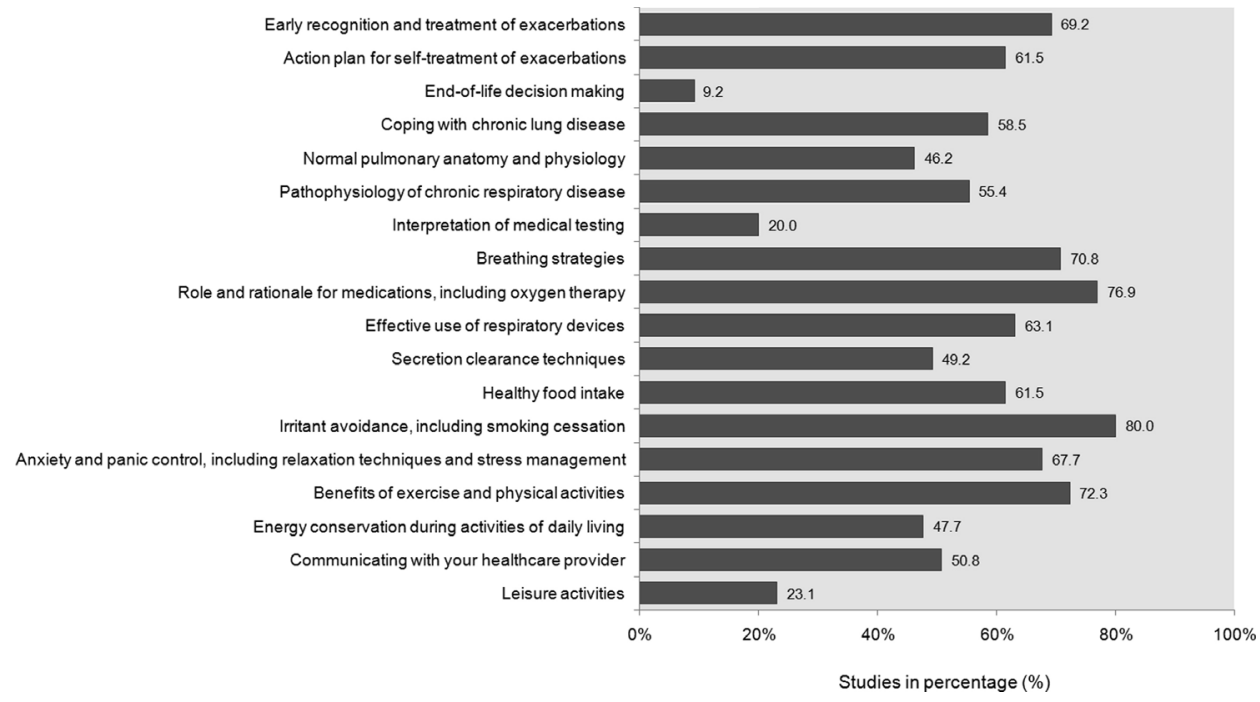

Figure 1.3 Percentage of studies that included each education topic. This Figure was published in Respiratory Medicine, Volume 107, Stoilkova, A., D.J. Janssen, and E.F. Wouters, Educational programmes in COPD management interventions: a systematic review. Pages 1637-1650, Copyright (C) 2013 Elsevier. ${ }^{85}$

While the goals of patient education are to enhance the patient's independence and the continuity of care in his or her own environment ${ }^{84}$, current practices of COPD education poorly address these issues. Furthermore, patients frequently experience a high level of anxiety, depression and impaired coping. ${ }^{86-88}$ In order to overcome these needs, changes in behavior, and particularly the acquisition of self-care skills, are required. ${ }^{89}$ This in turn requires the evolvement of the educational component from a traditional, teaching approach to the promotion of adaptive behavioral change, with a particular focus on collaborative self-management. ${ }^{10}$ Indeed, health behavior involves personal attributes such as beliefs, expectations, motives, values, perceptions and other cognitive elements; personality characteristics, including affective and emotional states and traits; and overt behavioral patterns, actions and habits that relate to health maintenance, restoration and improvement. ${ }^{90}$ Coping, defined as selecting and acting on the information derived from the individual's symptom recognitions and interpretation, is a challenge for patients with COPD. ${ }^{91}$ Coping profiles vary among COPD patients entering pulmonary rehabilitation with and without clinically relevant symptoms of anxiety and/or 
depression. ${ }^{86}$ Since coping styles are related to exercise tolerance, it is important to pay attention to coping styles in these patients. ${ }^{86}$ Personalized, tailored educational programs which take into account the individual patient's clinical, psychological, social and spiritual factors must become part of the comprehensive pulmonary rehabilitation program. ${ }^{92}$ Pulmonary rehabilitation needs to offer a dynamic, motivational, personcentered approach that helps the individual patient to understand his or her own personal risk factors, own barriers and facilitators to optimal self-management and the potential benefits of any given behavior change..$^{93}$

Both descriptions-exercise training and patient education-clearly illustrate ongoing problems in pulmonary rehabilitation when it comes to integrating ongoing developments in all the domains, thereby contributing to better health for the patient. In the absence of clear and uniform performance and process metrics and quality control to ensure appropriate standards for pulmonary rehabilitation, all programs, including those with minimal intervention, can be labeled as pulmonary rehabilitation, even when ignoring the fundamentals of this positive health intervention. 


\section{References}

1. Petty TL. Pulmonary Rehabilitation: A Personal Historical Perspective. Principles and Practice of Pulmonary Rehabilitation, ed. R. Casaburi and T.L. Petty. 1993, Philadelphia: Saunders.

2. Barach A, Bickerman $\mathrm{H}$, Beck $\mathrm{G}$. Advances in the treatment of non-tuberculous pulmonary disease. Bull NY Acad Med. 1952;28:353-84.

3. Hodgkin JE, et al. American Thoracic Society. Medical Section of the American Lung Association. Pulmonary rehabilitation. Am Rev Respir Dis. 1981;124(5):663-6.

4. Donner CF, Howard P. Pulmonary rehabilitation in chronic obstructive pulmonary disease (COPD) with recommendations for its use. Report of the European Respiratory Society Rehabilitation and Chronic Care Scientific Group (S.E.P.C.R. Rehabilitation Working Group). Eur Respir J. 1992;5(2):266-75.

5. Fishman AP. Pulmonary rehabilitation research. Am J Respir Crit Care Med. 1994;149(3 Pt 1):825-33.

6. Donner CF, Muir JF. Selection criteria and programmes for pulmonary rehabilitation in COPD patients. Rehabilitation and Chronic Care Scientific Group of the European Respiratory Society. Eur Respir J. 1997;10(3):744-57.

7. Britisch Thoracic Society (BTS), British Thoracic Society guidelines for the management of chronic obstructive pulmonary disease. The COPD Guidelines Group of the Standards of Care Committee of the BTS. Thorax. 1997;52 Suppl 5:S1-28.

8. American Thoracic Society (ATS), Official Statement of the American Thoracic Society. Pulmonary Rehabilitation - 1999. Am J Respir Crit Care Med. 1999;159:1666-82.

9. Nici $L$, et al. American Thoracic Society/European Respiratory Society statement on pulmonary rehabilitation. Am J Respir Crit Care Med. 2006;173(12):1390-413.

10. Spruit MA, et al. An official American Thoracic Society/European Respiratory Society statement: key concepts and advances in pulmonary rehabilitation. Am J Respir Crit Care Med. 2013;188(8):e13-64.

11. World Health Organization (WHO). https://www.who.int/publications/m/item/constitution-of-the-worldhealth-organization. 2006.

12. Wagner EH, Austin BT, Von Korff M. Organizing care for patients with chronic illness. Milbank Q. 1996; 74(4):511-44.

13. Leutz WN. Five laws for integrating medical and social services: lessons from the United States and the United Kingdom. Milbank Q. 1999;77(1):77-110, iv-v.

14. Grone O, Garcia-Barbero M, W.H.O. European Office for Integrated Health Care Services, Integrated care: a position paper of the WHO European Office for Integrated Health Care Services. Int J Integr Care. 2001;1:e21.

15. Spruit MA, et al. Differences in content and organisational aspects of pulmonary rehabilitation programmes. Eur Respir J. 2014;43(5):1326-37.

16. Singh SJ, et al. Learn from the past and create the future: the 2013 ATS/ERS statement on pulmonary rehabilitation. Eur Respir J. 2013;42(5):1169-74.

17. Troosters T, et al. The past, present and future of pulmonary rehabilitation. Respirology. 2019;24(9): 830-837.

18. Pauwels RA, et al. Global strategy for the diagnosis, management, and prevention of chronic obstructive pulmonary disease: National Heart, Lung, and Blood Institute and World Health Organization Global Initiative for Chronic Obstructive Lung Disease (GOLD): executive summary. Respir Care. 2001;46(8):798825.

19. Global Initiative for Chronic Obstructive Lung Disease (GOLD). Global Strategy for Prevention, Diagnosis and management of COPD. 2021.

20. Singh D, et al. Global Strategy for the Diagnosis, Management, and Prevention of Chronic Obstructive Lung Disease: the GOLD science committee report 2019. Eur Respir J. 2019;53(5).

21. Agusti A, MacNee W. The COPD control panel: towards personalised medicine in COPD. Thorax 2013; 68(7):687-90.

22. Agusti A, Gea J, Faner R. Biomarkers, the control panel and personalized COPD medicine. Respirology, 2016;21(1):24-33.

23. Agusti $A$, et al. Personalized respiratory medicine: exploring the horizon, addressing the issues. Summary of a BRN-AJRCCM workshop held in Barcelona on June 12, 2014. Am J Respir Crit Care Med. 2015;191(4):391-401. 
24. Vestbo J, et al. Global strategy for the diagnosis, management, and prevention of chronic obstructive pulmonary disease: GOLD executive summary. Am J Respir Crit Care Med. 2013;187(4):347-65.

25. von Leupoldt A, et al. The impact of anxiety and depression on outcomes of pulmonary rehabilitation in patients with COPD. Chest. 2011;140(3):730-6.

26. Mannino DM, et al. Prevalence and outcomes of diabetes, hypertension and cardiovascular disease in COPD. Eur Respir J. 2008;32(4):962-9.

27. Divo $\mathrm{M}$, et al. Comorbidities and risk of mortality in patients with chronic obstructive pulmonary disease. Am J Respir Crit Care Med. 2012;186(2):155-61.

28. Vanfleteren LE, et al. Clusters of comorbidities based on validated objective measurements and systemic inflammation in patients with chronic obstructive pulmonary disease. Am J Respir Crit Care Med. 2013;187(7):728-35.

29. Mesquita $\mathrm{R}$, et al. Objectively identified comorbidities in COPD: impact on pulmonary rehabilitation outcomes. Eur Respir J. 2015;46(2):545-8.

30. Vanfleteren L, et al. Management of chronic obstructive pulmonary disease beyond the lungs. Lancet Respir Med. 2016;4(11):911-24.

31. Agusti A, et al. Treatable traits: toward precision medicine of chronic airway diseases. Eur Respir J. 2016;47(2):410-9.

32. Jameson JL, Longo DL. Precision medicine--personalized, problematic, and promising. N Engl J Med. 2015;372(23):2229-34

33. Agusti A, et al. Characterisation of COPD heterogeneity in the ECLIPSE cohort. Respir Res. 2010;11:122

34. Duffy S, Weir M, Criner GJ. The complex challenge of chronic obstructive pulmonary disease. Lancet Respir Med. 2015;3(12):917-9.

35. Spruit MA, et al. Differential response to pulmonary rehabilitation in COPD: multidimensional profiling. Eur Respir J. 2015;46(6):1625-35.

36. Divo MJ, et al. COPD comorbidities network. Eur Respir J. 2015;46(3):640-50.

37. Agusti A. Phenotypes and disease characterization in chronic obstructive pulmonary disease. Toward the extinction of phenotypes? Ann Am Thorac Soc. 2013;10 Suppl:S125-30.

38. Agusti A. The path to personalised medicine in COPD. Thorax. 2014;69(9):857-64.

39. Wouters EF, et al. Personalized medicine and chronic obstructive pulmonary disease. Curr Opin Pulm Med. 2017;23(3):241-6.

40. Frey U, Suki B. Complexity of chronic asthma and chronic obstructive pulmonary disease: implications for risk assessment, and disease progression and control. Lancet. 2008;372(9643):1088-99.

41. Pulmonary rehabilitation: joint ACCP/AACVPR evidence-based guidelines. ACCP/AACVPR Pulmonary Rehabilitation Guidelines Panel. American College of Chest Physicians. American Association of Cardiovascular and Pulmonary Rehabilitation. Chest. 1997;112(5):1363-96.

42. Ries AL, ACCP/AACVPR evidence-based guidelines for pulmonary rehabilitation. Round 3: another step forward. J Cardiopulm Rehabil Prev. 2007;27(4):233-6.

43. Bolton CE, et al. British Thoracic Society guideline on pulmonary rehabilitation in adults. Thorax. 2013; 68 Suppl 2:ii1-30.

44. Casaburi $R$, et al. Reductions in exercise lactic acidosis and ventilation as a result of exercise training in patients with obstructive lung disease. Am Rev Respir Dis. 1991;143(1):9-18.

45. Casaburi R, et al. Physiologic benefits of exercise training in rehabilitation of patients with severe chronic obstructive pulmonary disease. Am J Respir Crit Care Med. 1997;155(5):1541-51.

46. Curtis JR, Deyo RA, Hudson LD. Pulmonary rehabilitation in chronic respiratory insufficiency. 7. Healthrelated quality of life among patients with chronic obstructive pulmonary disease. Thorax. 1994;49(2): 162-70.

47. Qaseem A, et al. Diagnosis and management of stable chronic obstructive pulmonary disease: a clinical practice guideline update from the American College of Physicians, American College of Chest Physicians, American Thoracic Society, and European Respiratory Society. Ann Intern Med. 2011; 155(3):179-91.

48. Tinetti ME, Naik AD, Dodson JA. Moving From Disease-Centered to Patient Goals-Directed Care for Patients With Multiple Chronic Conditions: Patient Value-Based Care. JAMA Cardiol. 2016;1(1):9-10.

49. Spruit MA, Wouters EFM. Organizational aspects of pulmonary rehabilitation in chronic respiratory diseases. Respirology. 2019;24(9):838-43.

50. Sackett DL, et al. Evidence based medicine: what it is and what it isn't. BMJ. 1996;312(7023):71-2. 
51. Evidence-Based Medicine Working G. Evidence-based medicine. A new approach to teaching the practice of medicine. JAMA. 1992;268(17):2420-5.

52. What is Cochrane? https://www.cochrane.org/about-us. [cited 2021].

53. Lacasse $Y$, et al. Meta-analysis of respiratory rehabilitation in chronic obstructive pulmonary disease. Lancet. 1996;348(9035):1115-9.

54. McCarthy B, et al. Pulmonary rehabilitation for chronic obstructive pulmonary disease. Cochrane Database Syst Rev. 2015;(2):CD003793.

55. Lacasse $Y$, et al. Pulmonary rehabilitation for chronic obstructive pulmonary disease. Cochrane Database Syst Rev/ 2001.

56. Lacasse $Y$, et al. Pulmonary rehabilitation for chronic obstructive pulmonary disease. Cochrane Database Syst Rev/ 2006;(4):CD003793.

57. Lacasse $Y$, et al. This Cochrane Review is closed: deciding what constitutes enough research and where next for pulmonary rehabilitation in COPD. Cochrane Database Syst Rev. 2015;(11):ED000107.

58. Pauwels RA. National and international guidelines for COPD: the need for evidence. Chest. 2000;117(2 Suppl):20S-2S.

59. Timmermans S, Berg M. The Gold Standard: The Challenge Of Evidence-Based Medicine. 2003: Temple University Press.

60. Greenhalgh T, et al. Evidence based medicine: a movement in crisis? BMJ. 2014;348:g3725.

61. Weatherall DJ. Science and the Quiet Art. Medical Research and Patient Care. 1995: Oxford University Press.

62. Tiep BL. Pulmonary Rehabilitation Program Organization. Principles and Practice of Pulmonary Rehabilitation, ed. R. Casaburi and T.L. Petty. 1993, Philadelphia: Saunders.

63. Francis G. Medicine: art or science? Lancet. 2020;395(10217):24-5.

64. Van der Weyden MB. The essence of the art of medicine. Med J Aust. 2006;185(4).

65. Philpot CR. The essence of the art of medicine. Med J Aust. 2006;185(11-12):672.

66. Haas F, Salazar-Schicchi J, Axen K. Desensitization to Dyspnea in Chronic Obstructive Pulmonary Disease. Principles and Practice of Pulmonary Rehabilitation, ed. R. Casaburi and T.L. Petty. 1993, Philadelphia: Saunders.

67. Ries AL, et al. Effects of pulmonary rehabilitation on physiologic and psychosocial outcomes in patients with chronic obstructive pulmonary disease. Ann Intern Med. 1995;122(11):823-32.

68. Skeletal muscle dysfunction in chronic obstructive pulmonary disease. A statement of the American Thoracic Society and European Respiratory Society. Am J Respir Crit Care Med. 1999;159(4 Pt 2):S1-40.

69. Curtis JR, Martin DP, Martin TR. Patient-assessed health outcomes in chronic lung disease: what are they, how do they help us, and where do we go from here? Am J Respir Crit Care Med. 1997;156(4 Pt 1):1032-9.

70. De Vries J, Drent M. Quality of life and health-related quality of life measures. Respir Med 2001;95(2): 159-60.

71. Karimi M, Brazier J. Health, Health-Related Quality of Life, and Quality of Life: What is the Difference? Pharmacoeconomics. 2016;34(7):645-9.

72. Ekstrom M, Sundh J, Larsson K. Patient reported outcome measures in chronic obstructive pulmonary disease: Which to use? Expert Rev Respir Med. 2016;10(3):351-62.

73. Health U.S.D.o, et al. Guidance for industry: patient-reported outcome measures: use in medical product development to support labeling claims: draft guidance. Health Qual Life Outcomes. 2006';4: 79.

74. Benzo RP, et al. Emotional Intelligence: A Novel Outcome Associated with Wellbeing and SelfManagement in Chronic Obstructive Pulmonary Disease. Ann Am Thorac Soc. 2016;13(1):10-6.

75. Meis JJ, et al. A qualitative assessment of COPD patients' experiences of pulmonary rehabilitation and guidance by healthcare professionals. Respir Med. 2014;108(3):500-10.

76. Brien SB, Lewith GT, Thomas M. Patient coping strategies in COPD across disease severity and quality of life: a qualitative study. NPJ Prim Care Respir Med. 2016;26:16051.

77. Conner M, Norman P. Health behaviour: Current issues and challenges. Psychol Health. 2017;32(8): 895906.

78. Garvey C, et al. Pulmonary Rehabilitation Exercise Prescription in Chronic Obstructive Pulmonary Disease: Review of Selected Guidelines: An official statement from the American Association of Cardiovascular and Pulmonary Rehabilitation. J Cardiopulm Rehabil Prev. 2016;36(2):75-83. 
79. Gosselink R, Troosters T, Decramer M. Exercise training in COPD patients: the basic questions. Eur Respir J. 1997;10(12):2884-91.

80. Holland $A E$, et al. Home-based rehabilitation for COPD using minimal resources: a randomised, controlled equivalence trial. Thorax. 2017;72(1):57-65.

81. Wuytack F, et al. Comparison of outpatient and home-based exercise training programmes for COPD: A systematic review and meta-analysis. Respirology 2018;23(3):272-83.

82. Casaburi R. Whither pulmonary rehabilitation? Will alternative modes help or hurt? Eur Respir J. 2018; 52(4).

83. Spruit MA, et al. Profiling of Patients with COPD for Adequate Referral to Exercise-Based Care: The Dutch Model. Sports Med. 2020;50(8):1421-9.

84. Dreeben O. Patient education in rehabilitation 2010: Jones and Bartlett Publisher, LLC.

85. Stoilkova A, Janssen DJ, Wouters EF. Educational programmes in COPD management interventions: a systematic review. Respir Med. 2013;107(11):1637-50.

86. Stoilkova A, et al. The relationship between coping styles and clinical outcomes in patients with COPD entering pulmonary rehabilitation. COPD. 2013;10(3):316-23.

87. Stoilkova A, et al. Coping styles in patients with COPD before and after pulmonary rehabilitation. Respir Med. 2013;107(6):825-33.

88. Stoilkova-Hartmann A, et al. Differences in change in coping styles between good responders, moderate responders and non-responders to pulmonary rehabilitation. Respir Med. 2015;109(12): 1540-5.

89. Rice K, et al. Collaborative self-management and behavioral change. Clin Chest Med. 2014;35(2): 337-51.

90. Gochman DS. Handbook of Health Behavior Research I. 1997, New York, NY, United States: Springer Science+Business Media. 506 pages.

91. O'Neill ES. Illness representations and coping of women with chronic obstructive pulmonary disease: a pilot study. Heart Lung 2002;31(4):295-302.

92. Almagro $P$, Castro A. Helping COPD patients change health behavior in order to improve their quality of life. Int J Chron Obstruct Pulmon Dis. 2013;8:335-45.

93. Stoilkova-Hartmann A, et al. COPD patient education and support - Achieving patient-centredness. Patient Educ Couns. 2018;101(11):2031-6. 


\section{Supplemental material}

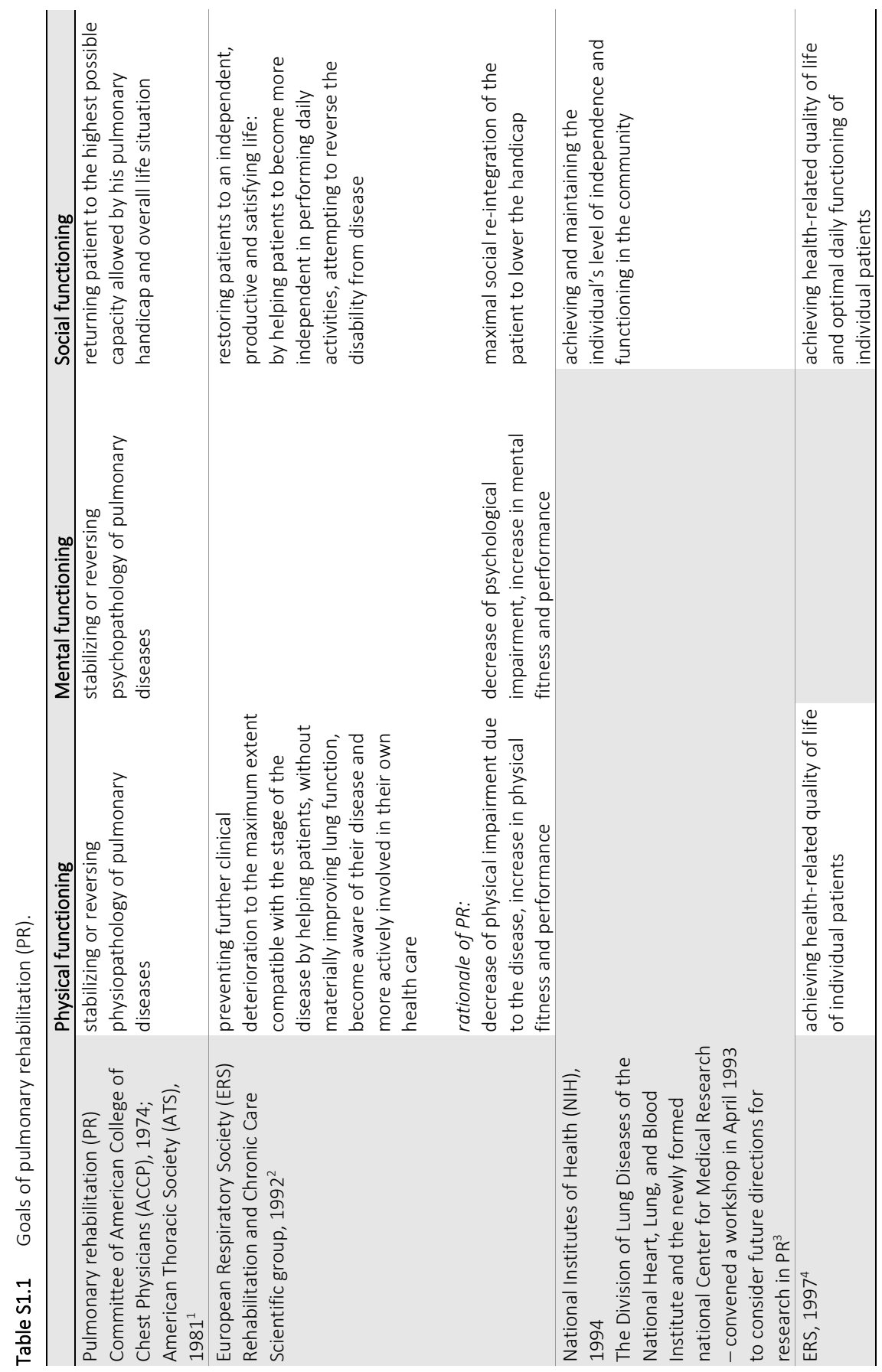




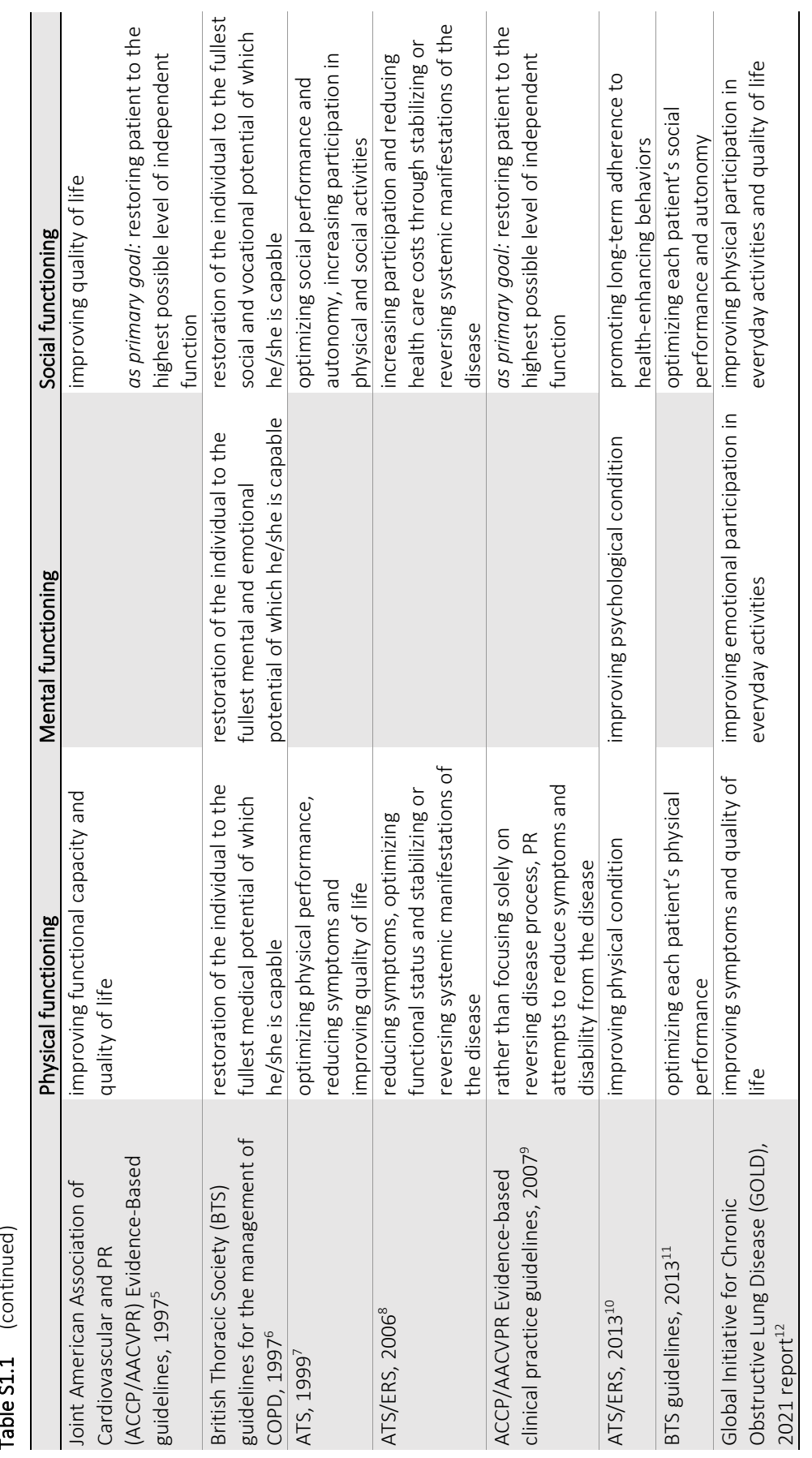




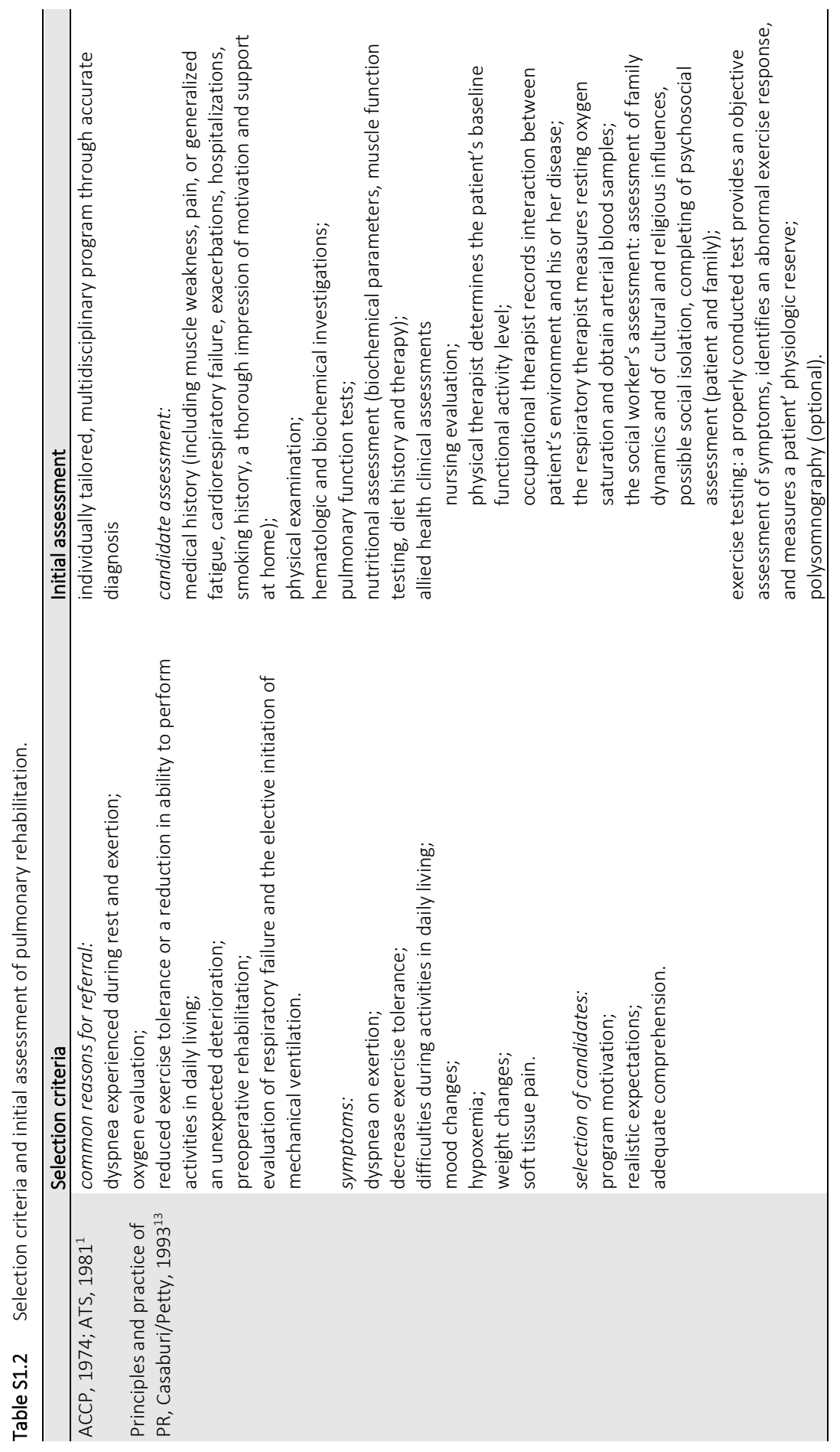




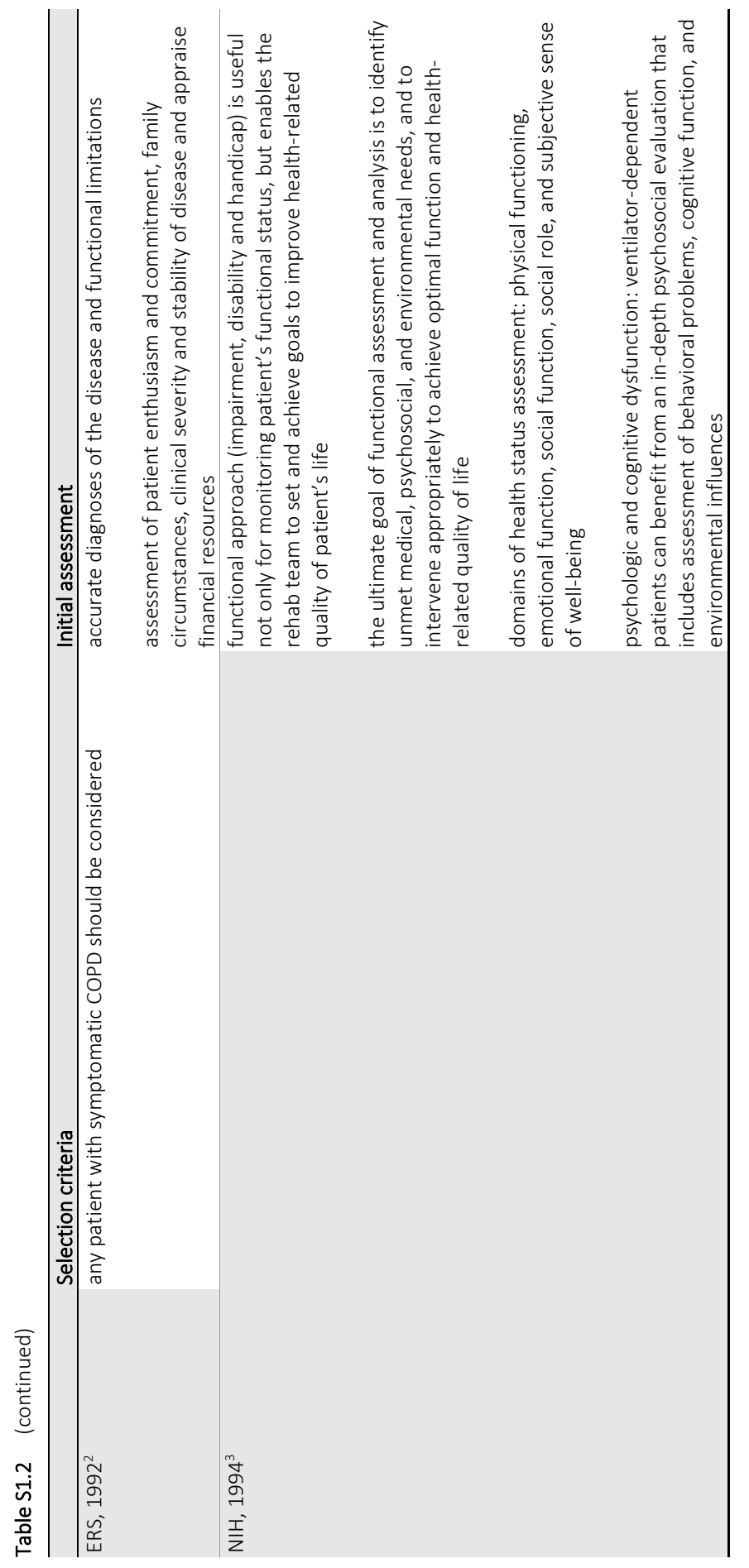




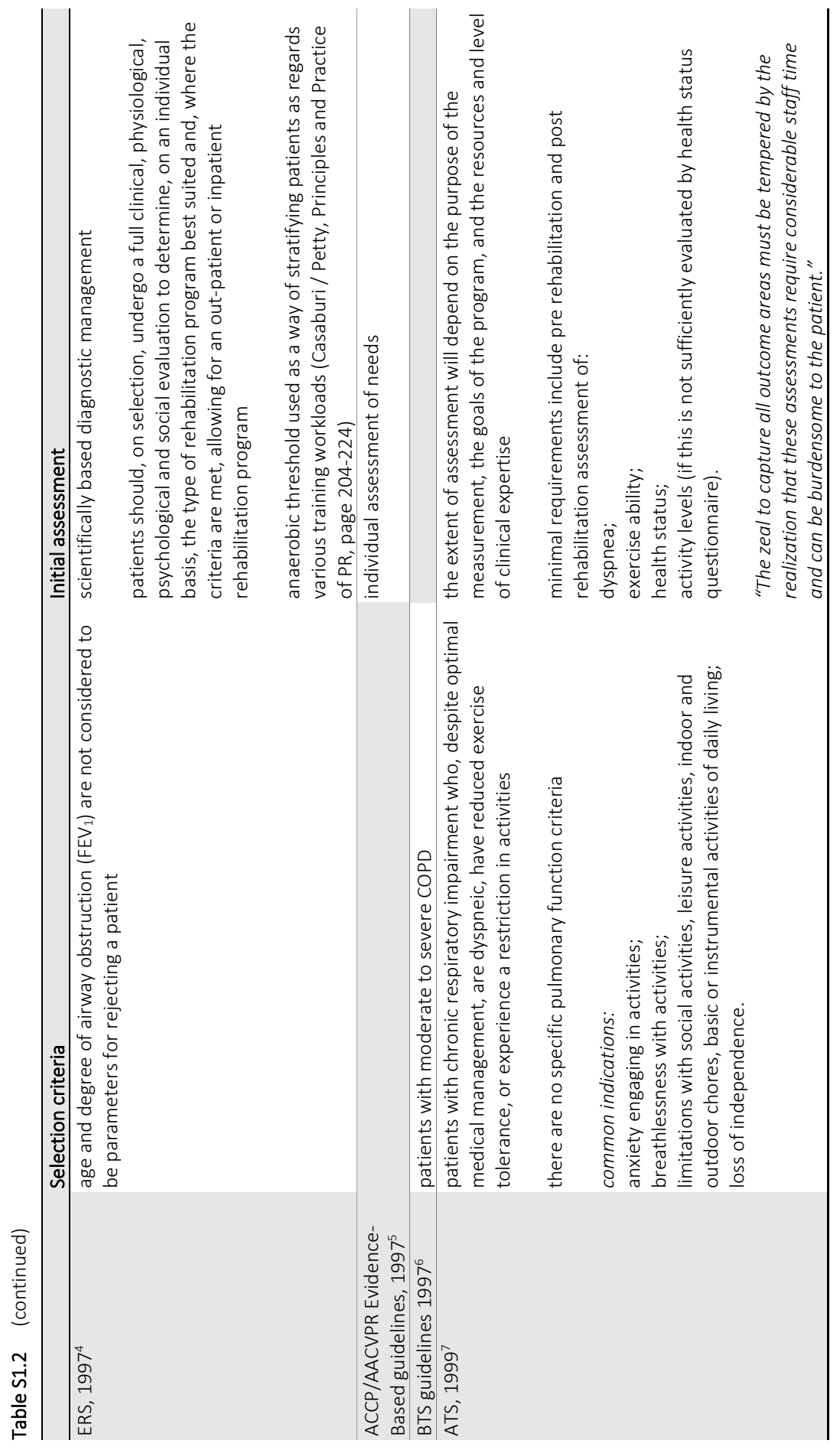




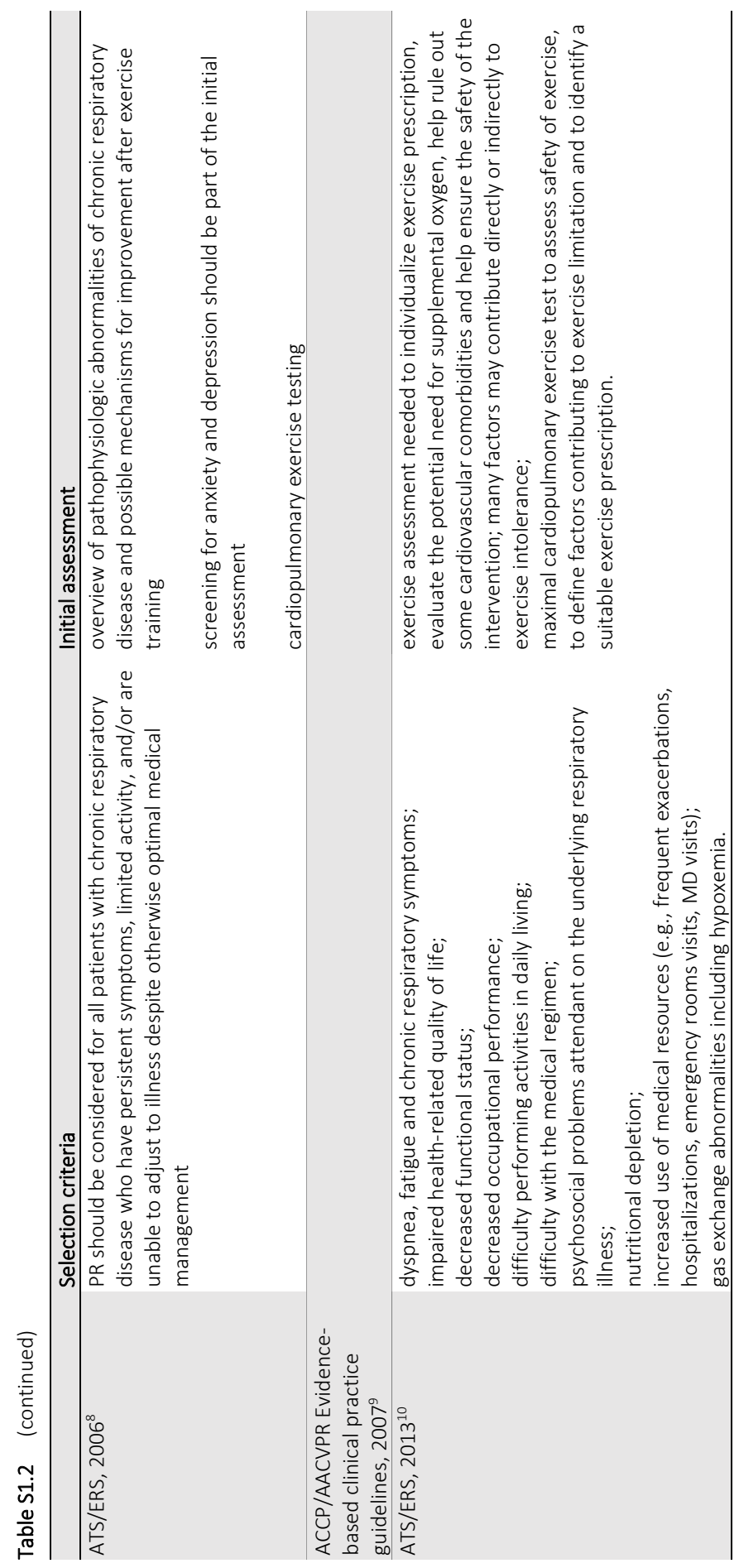




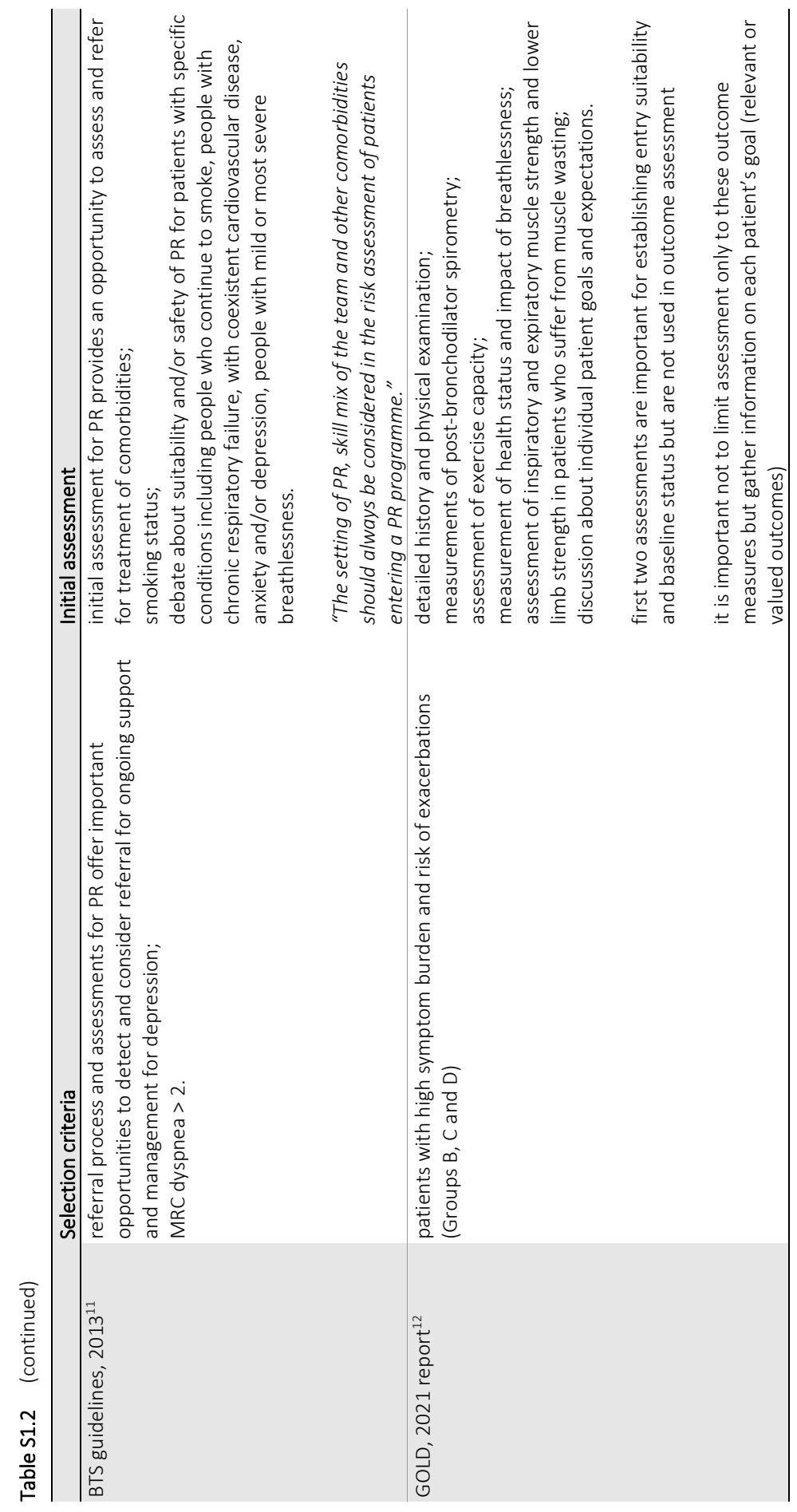




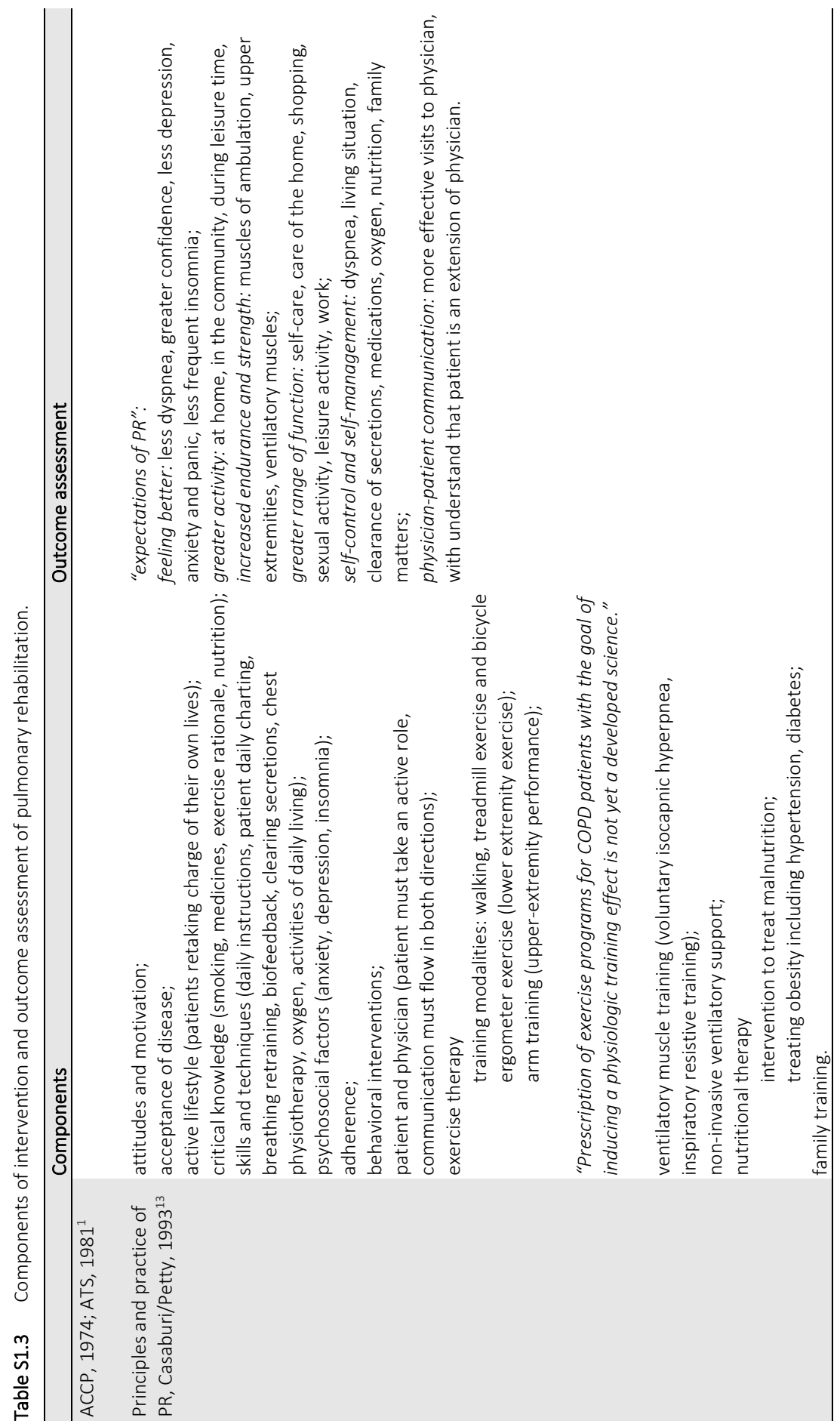




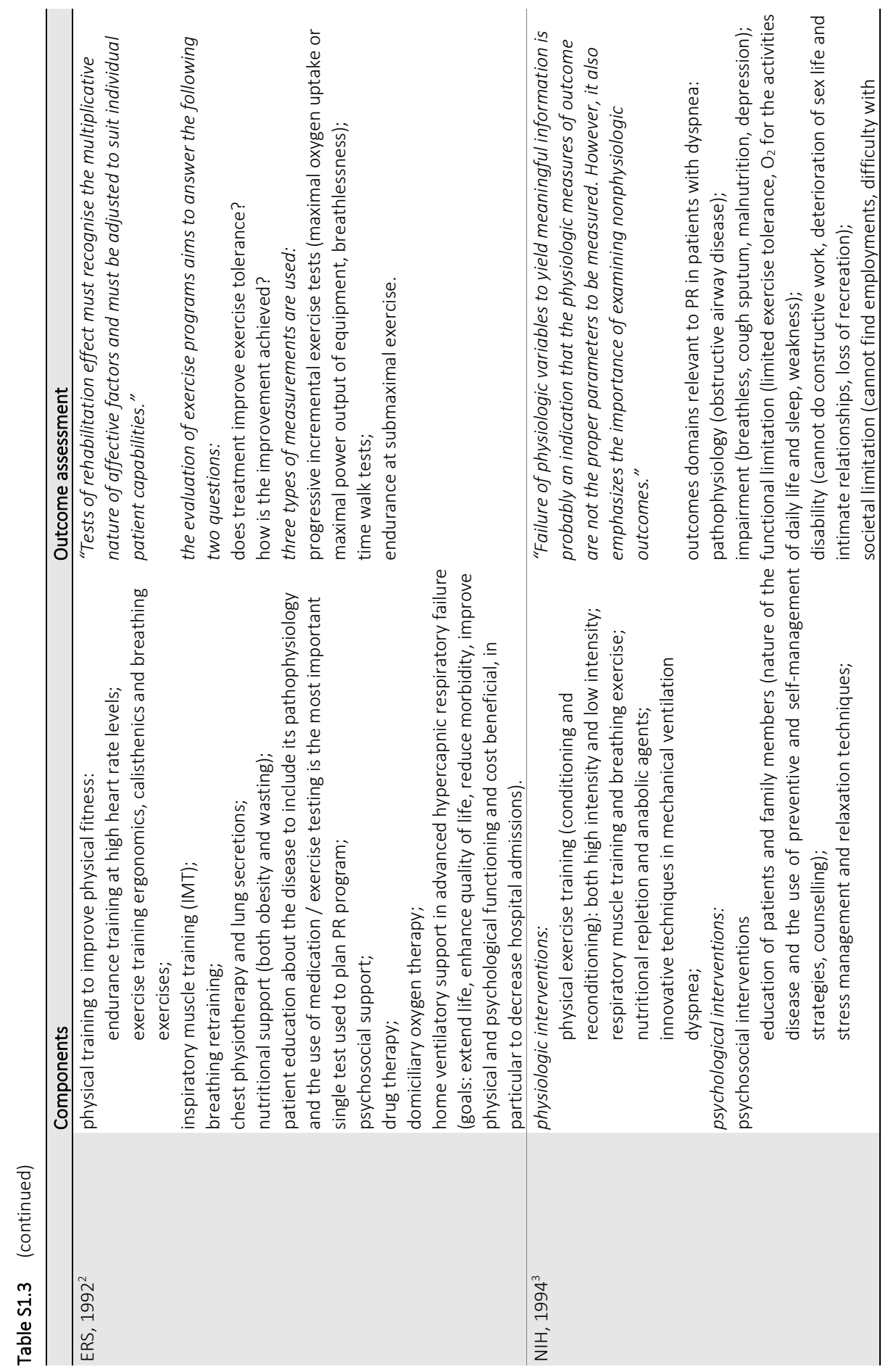




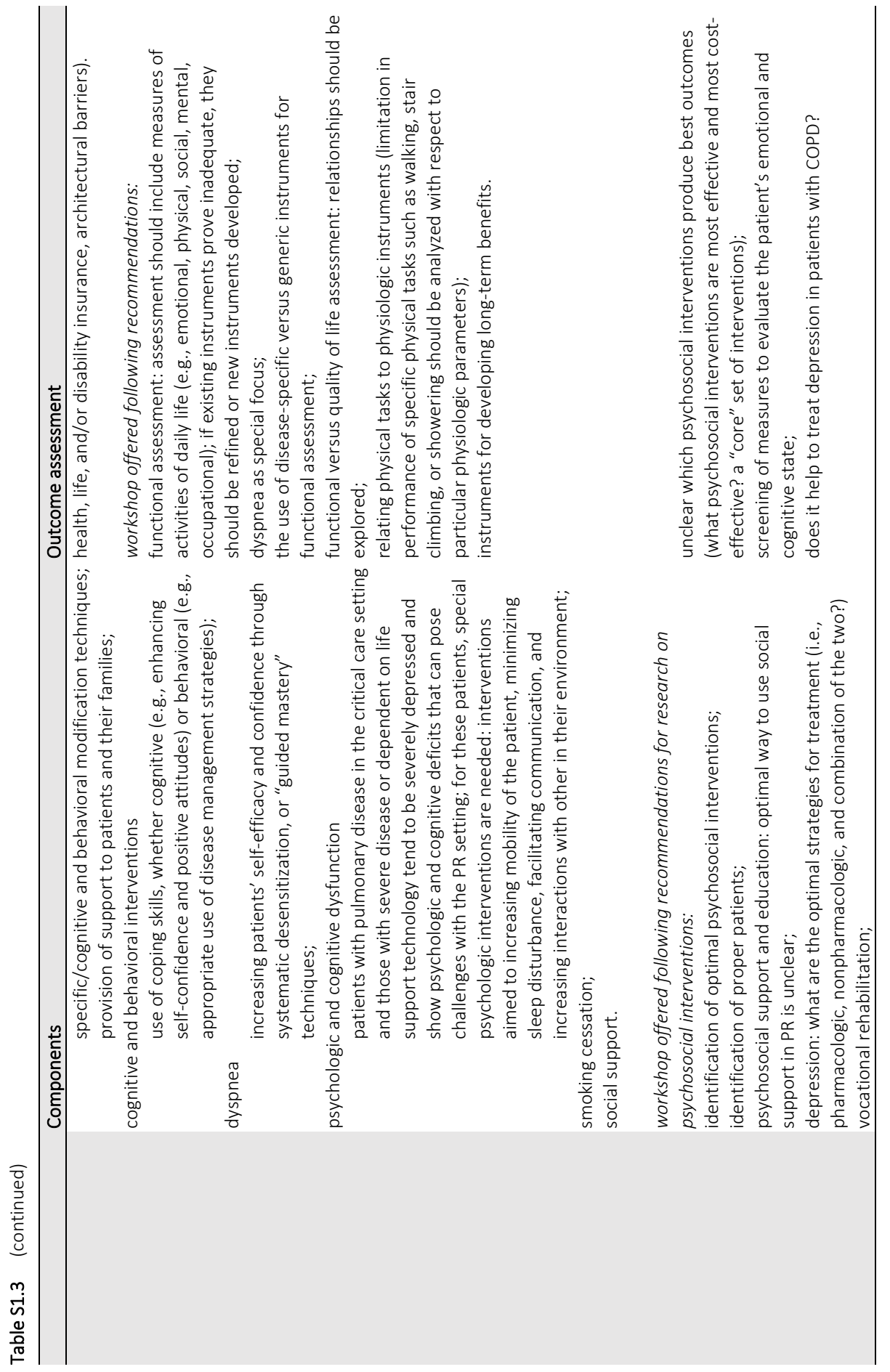




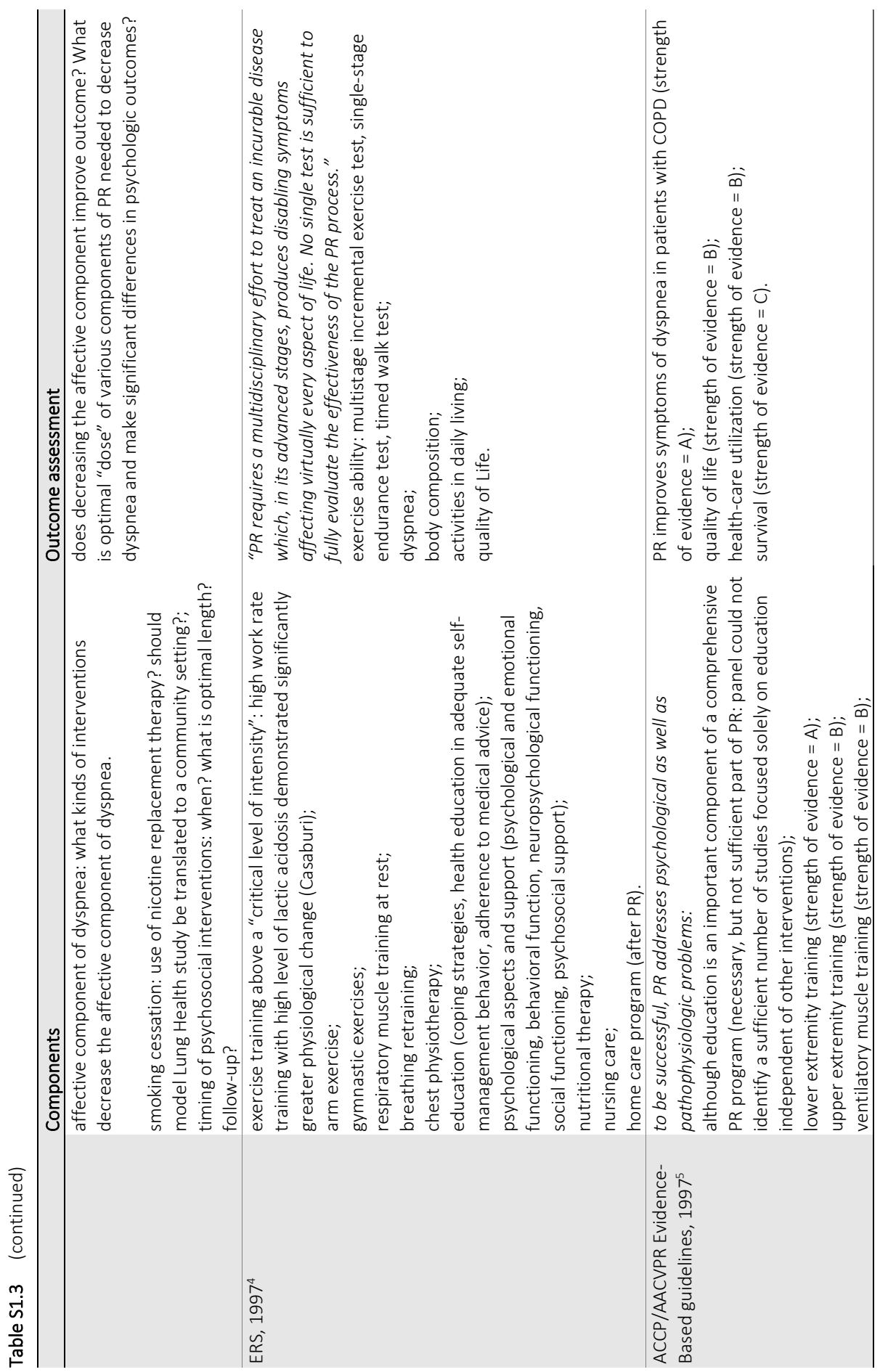




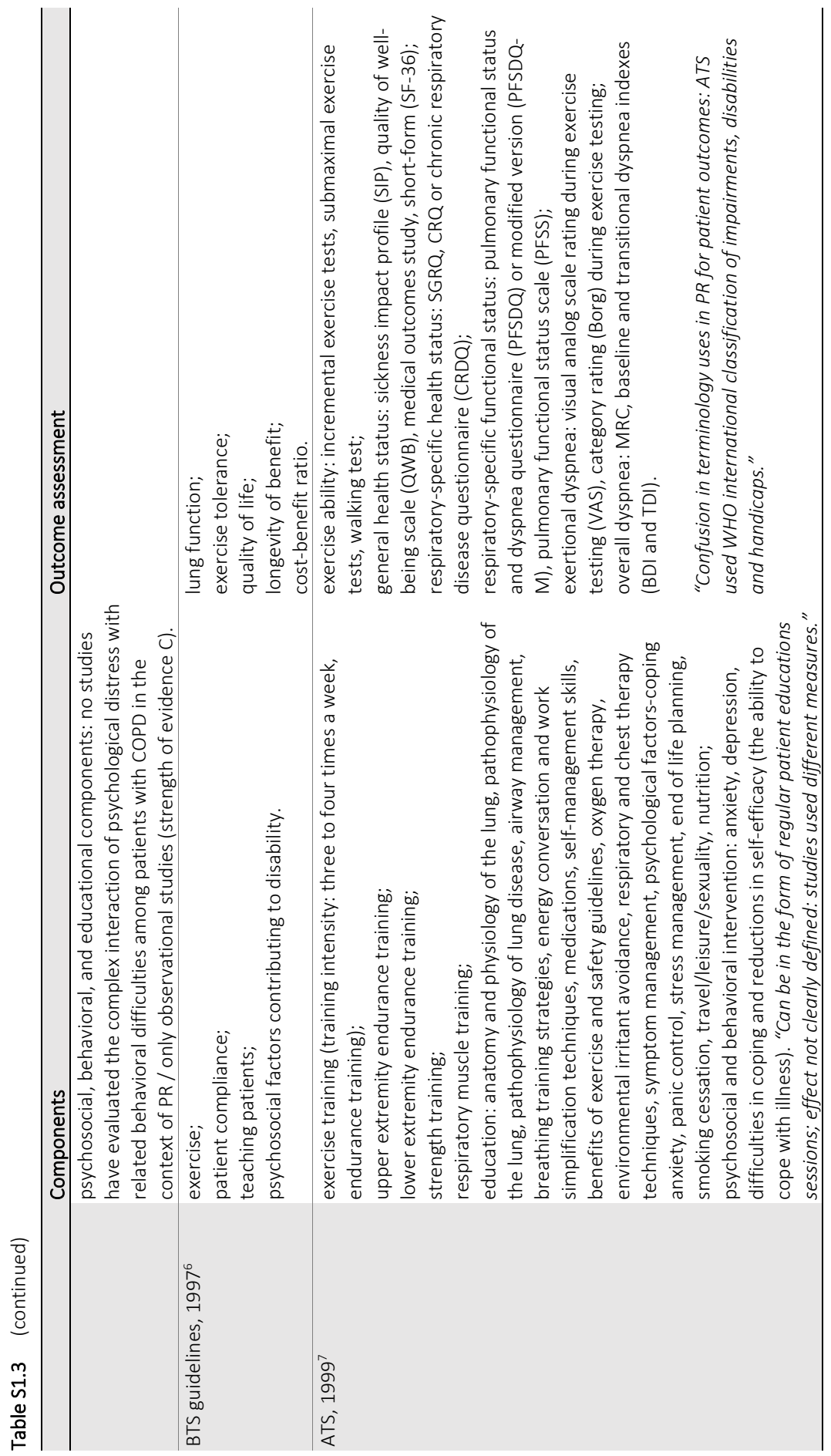




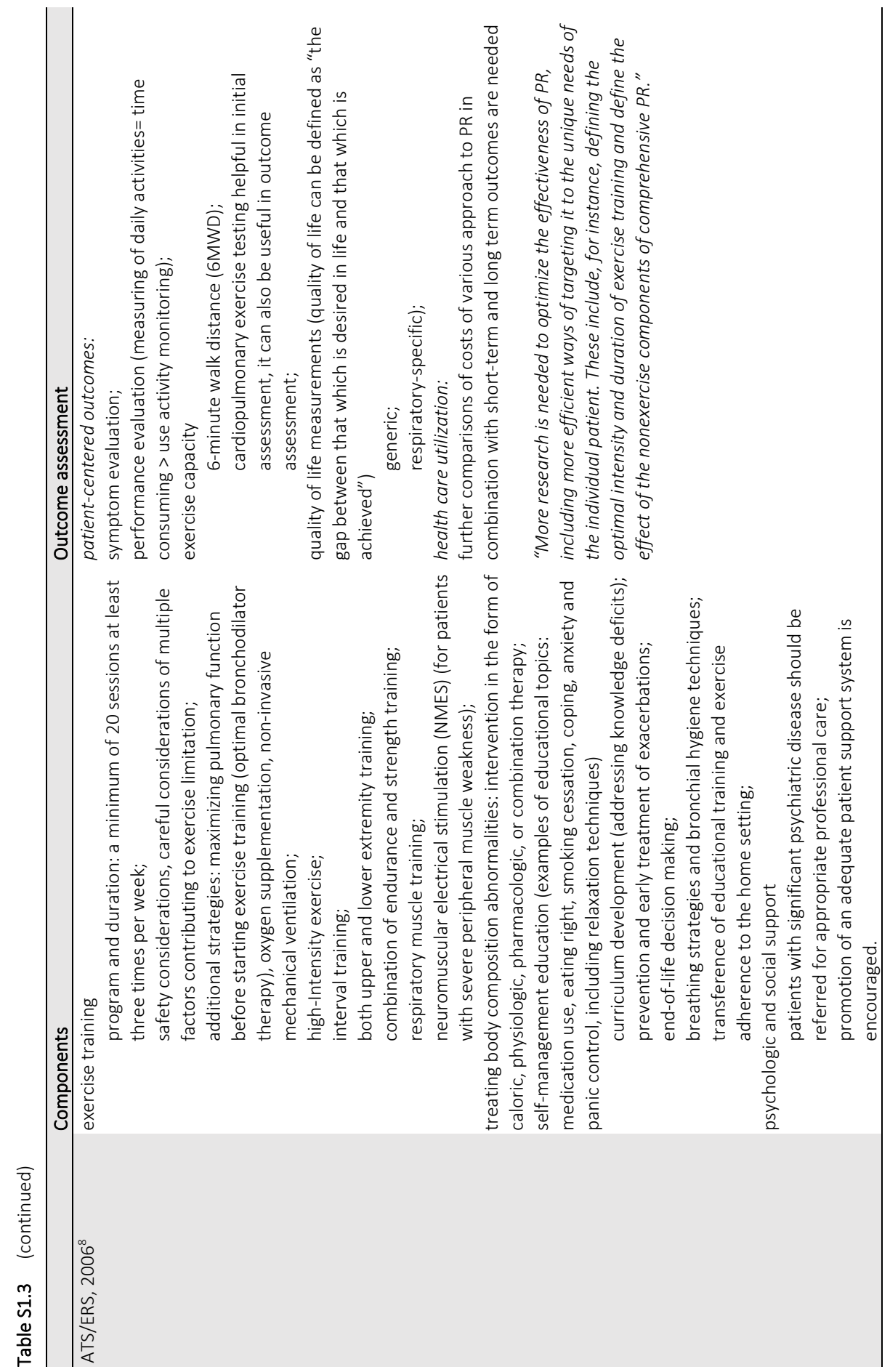




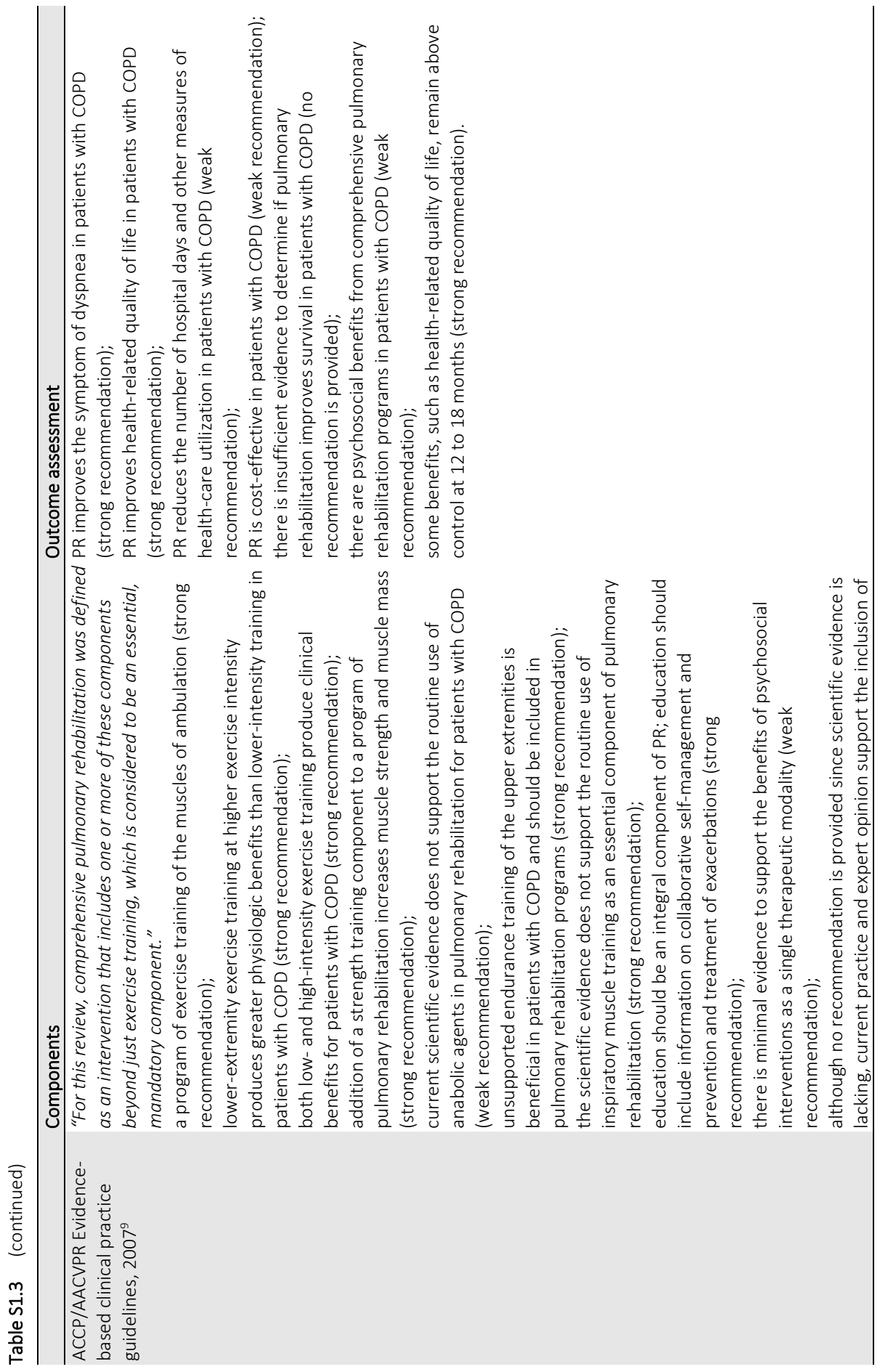




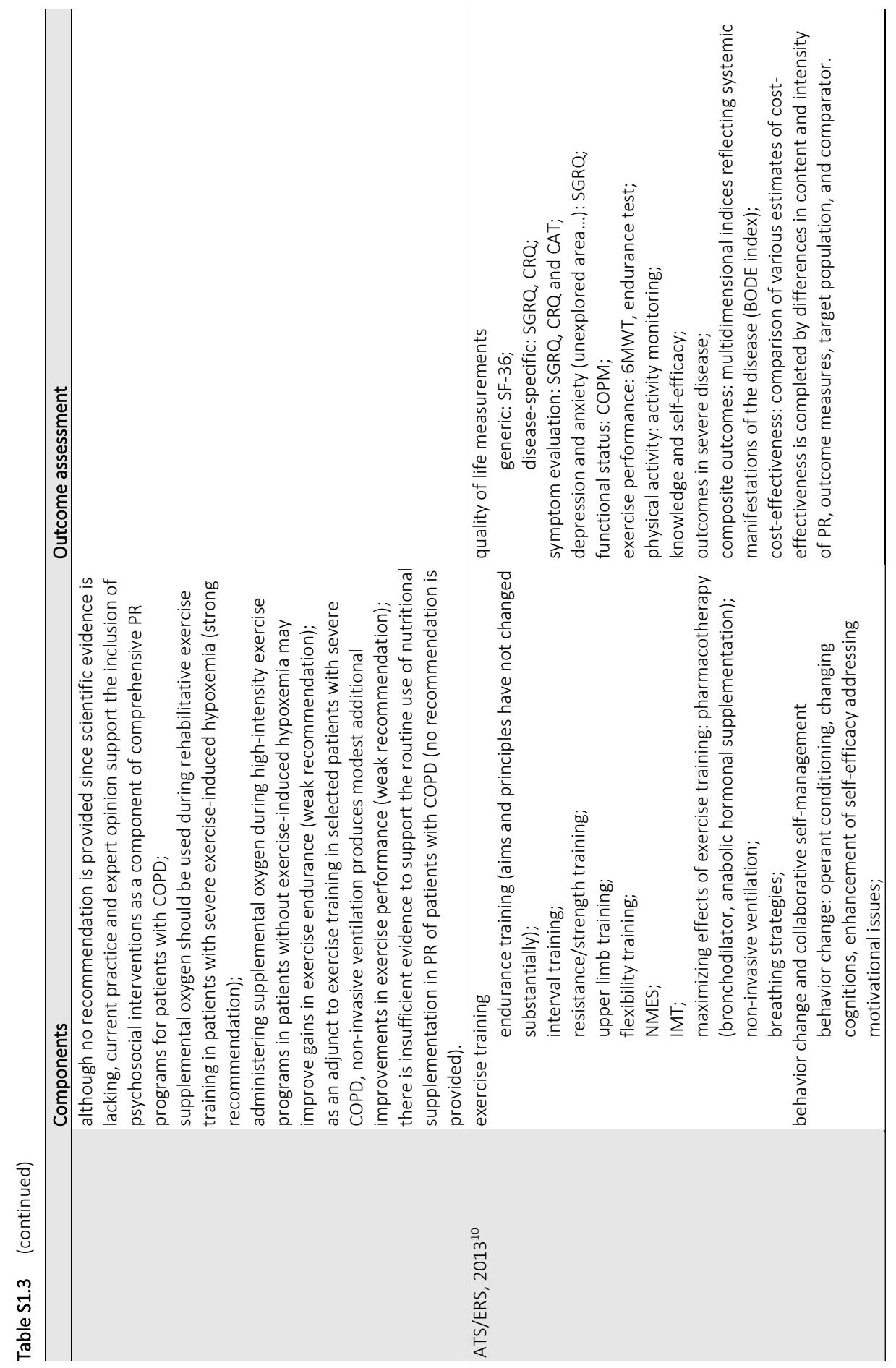




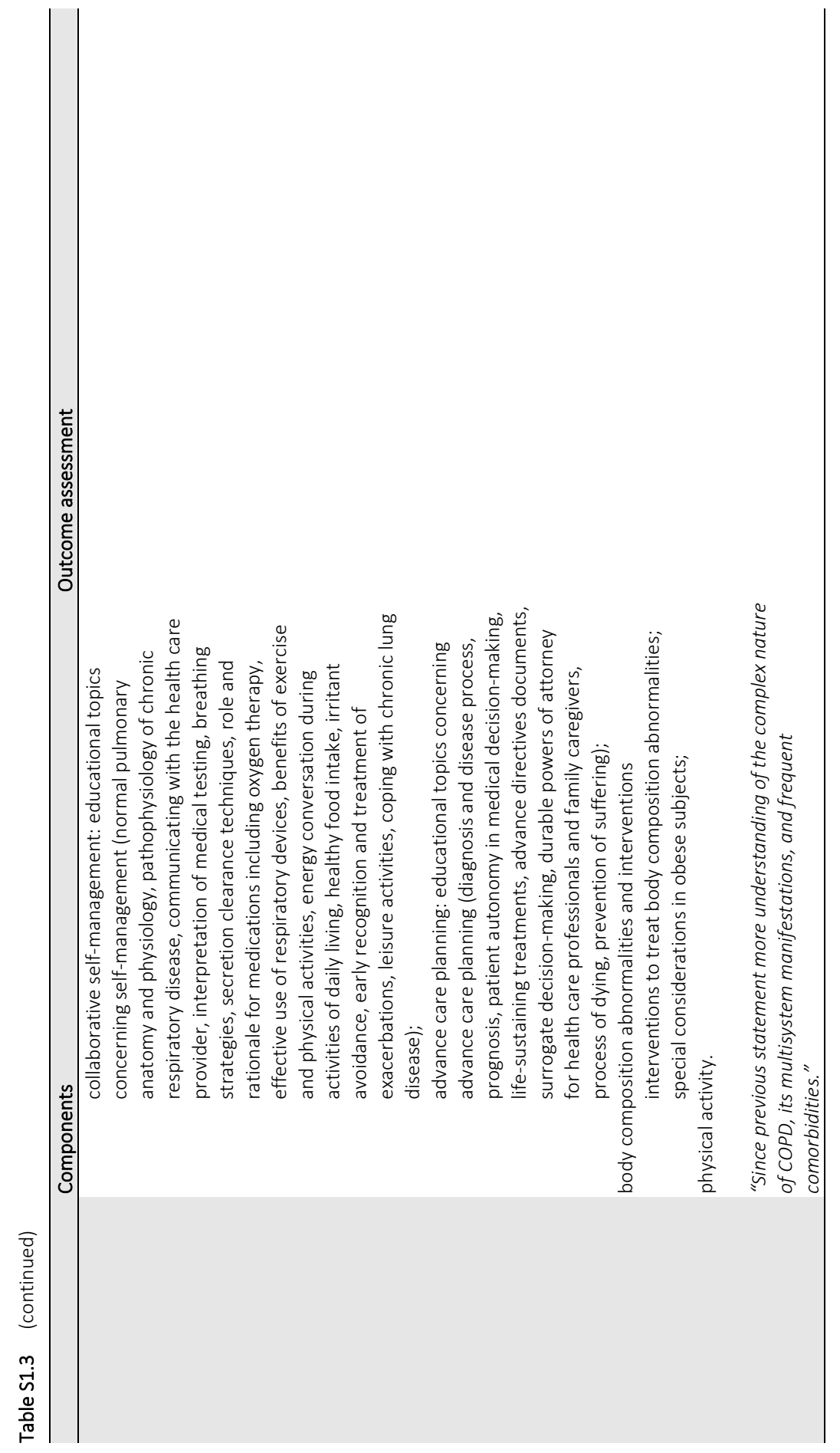




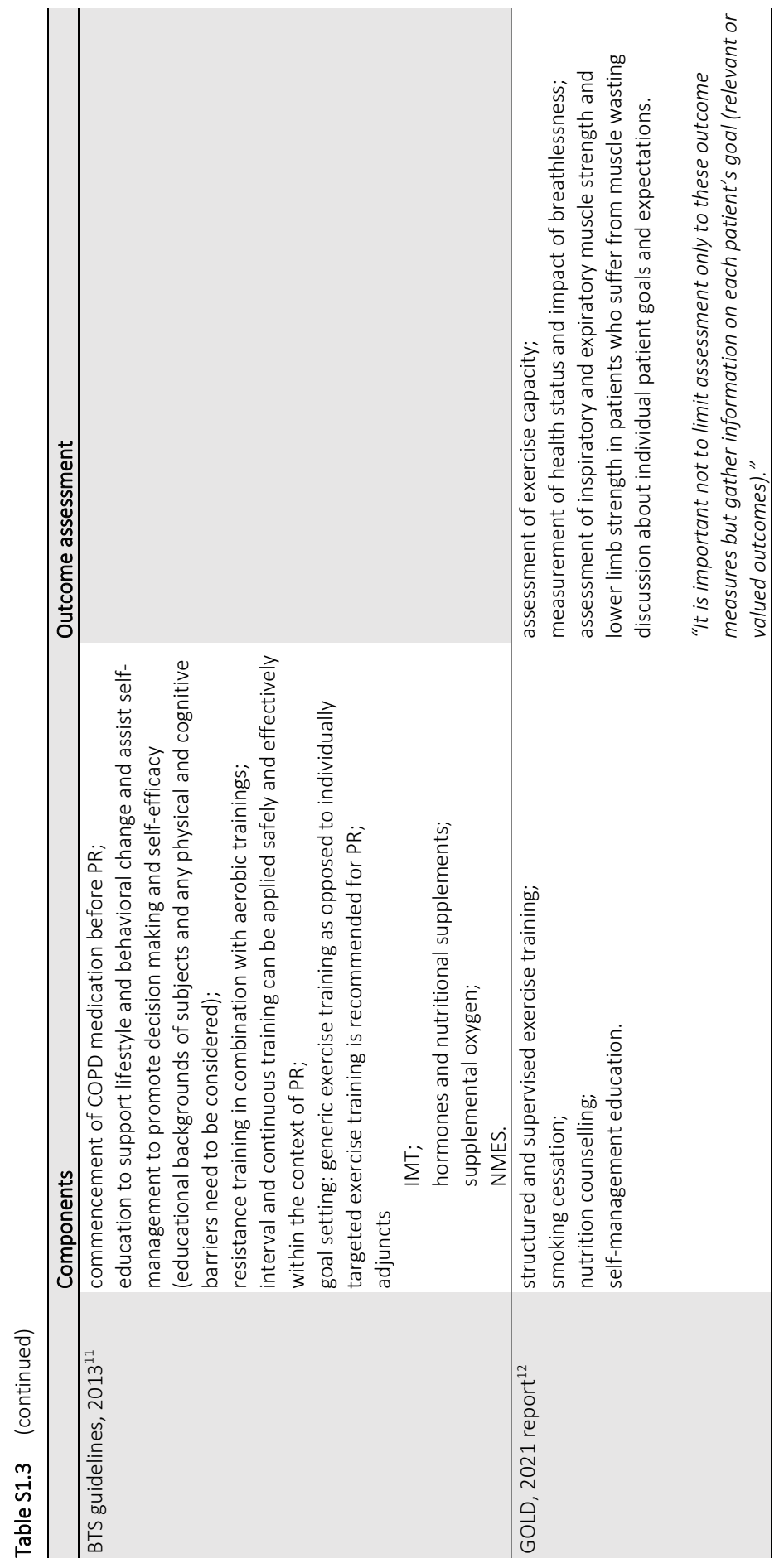




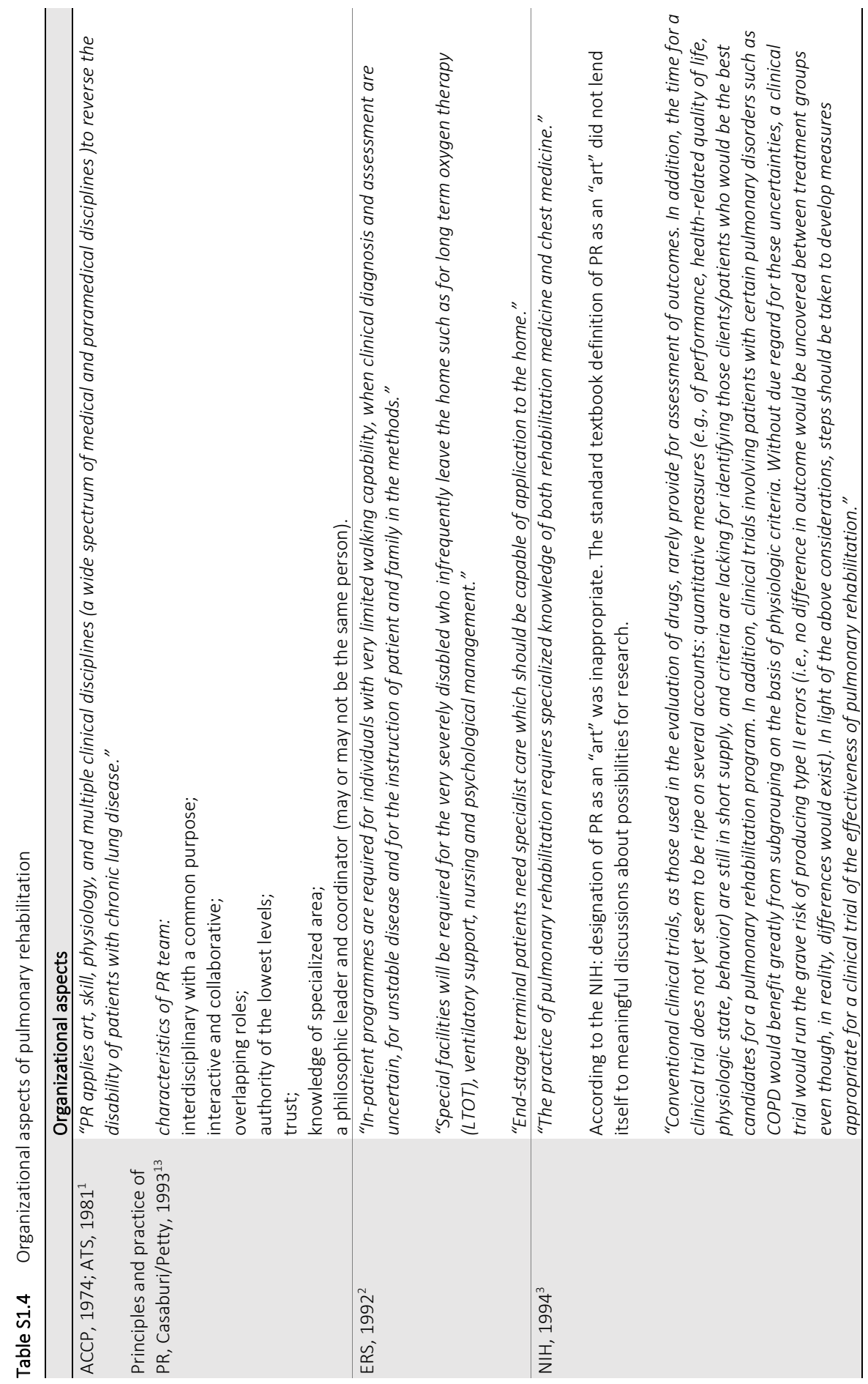




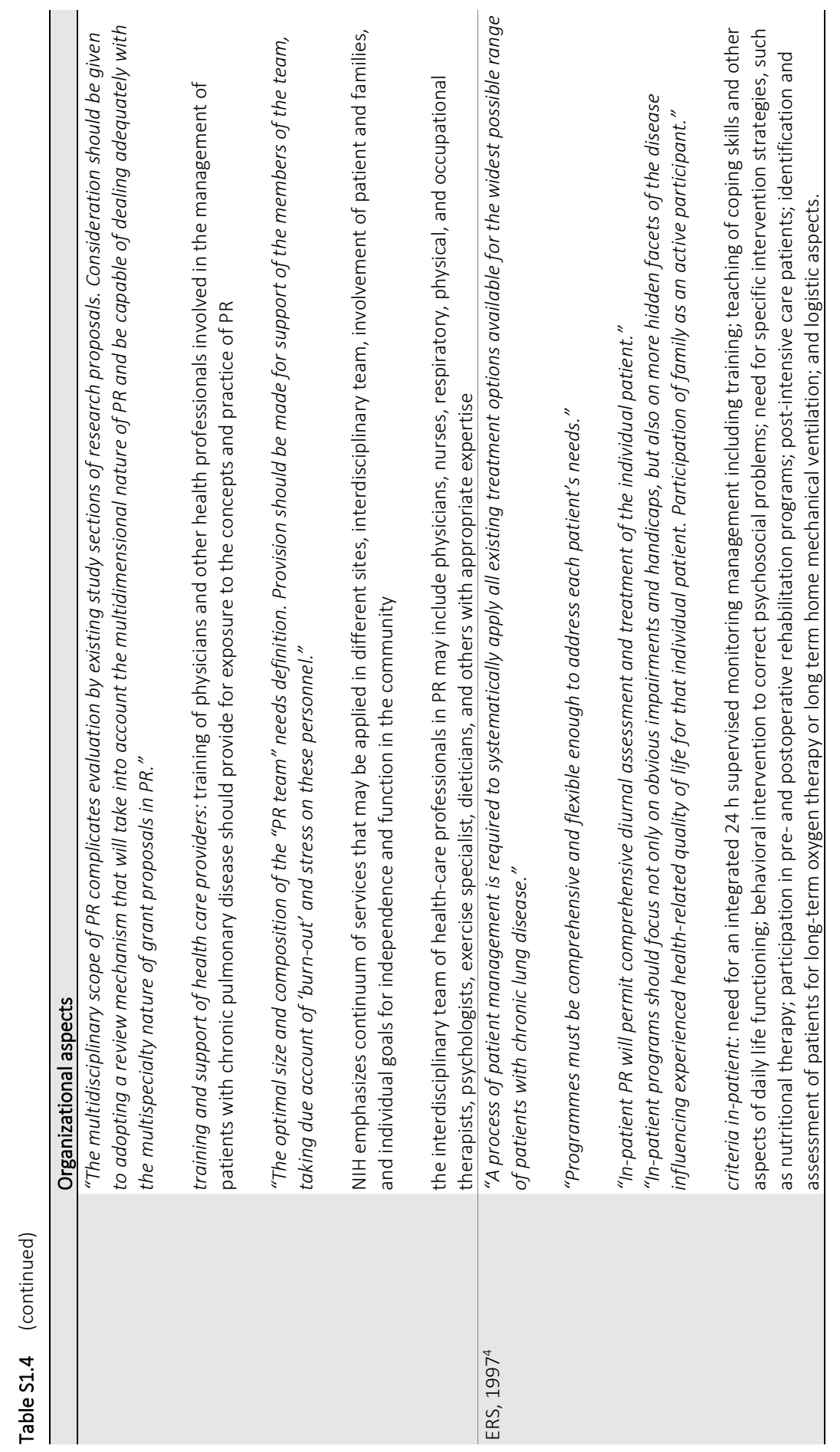




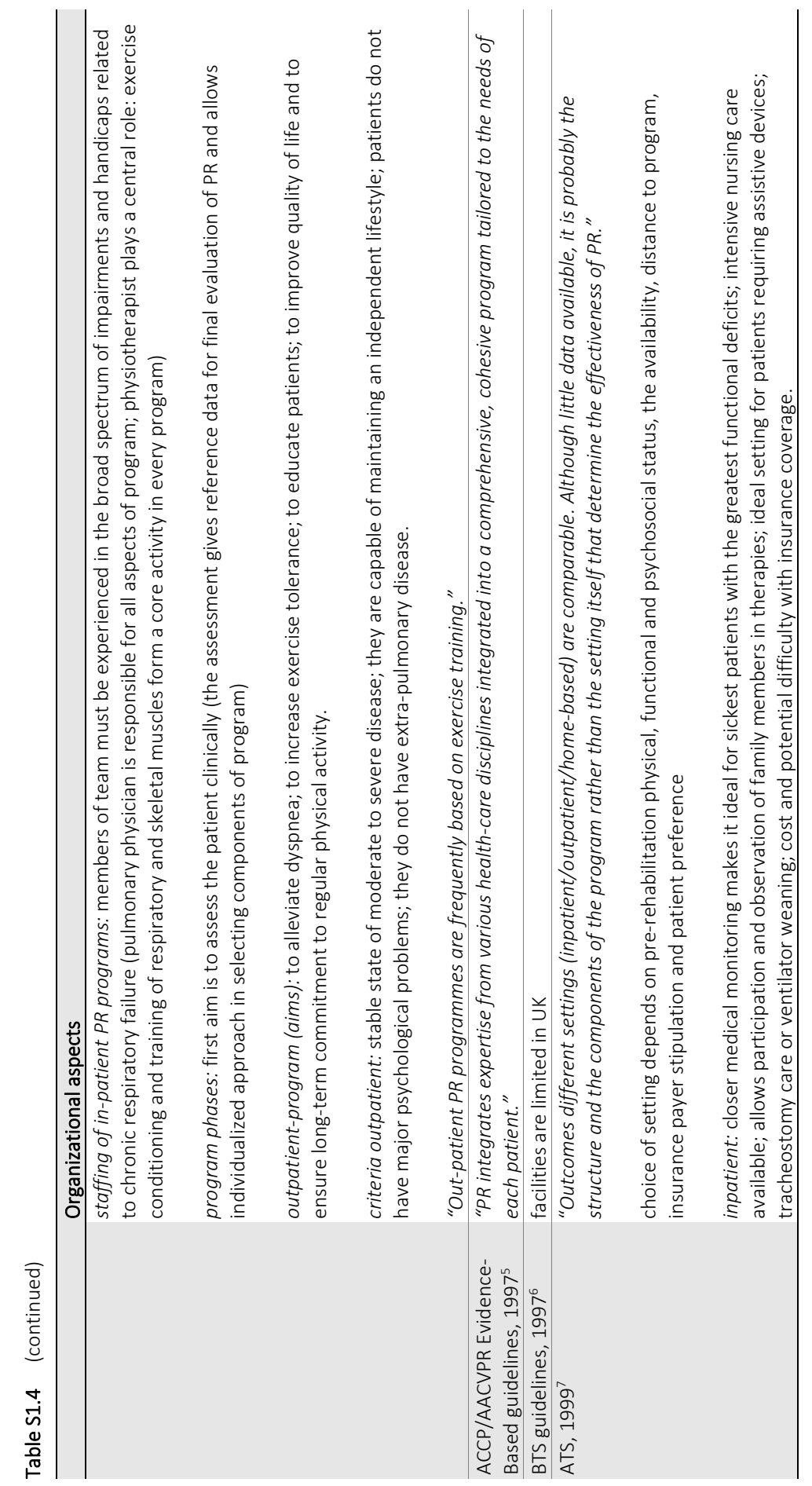




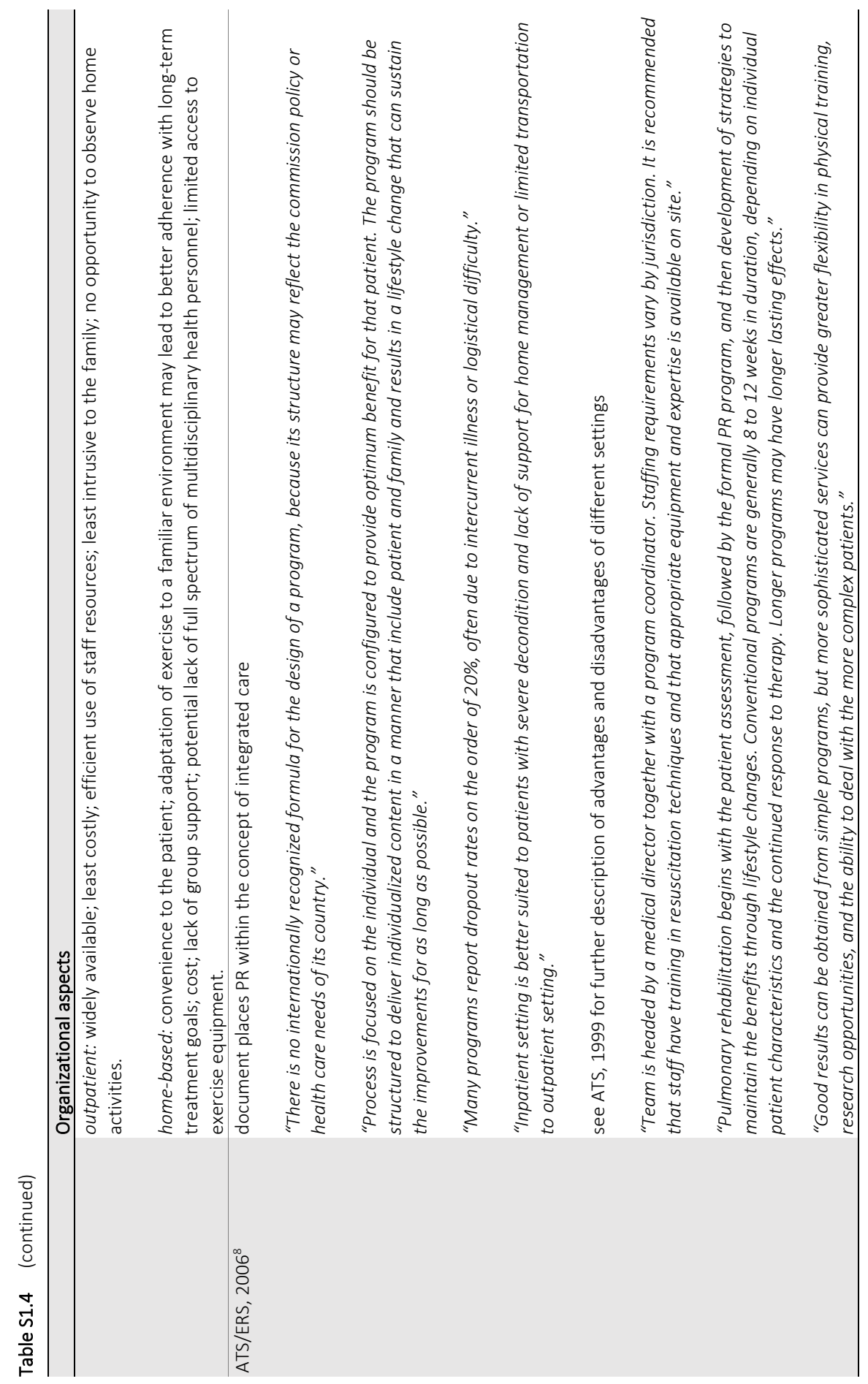




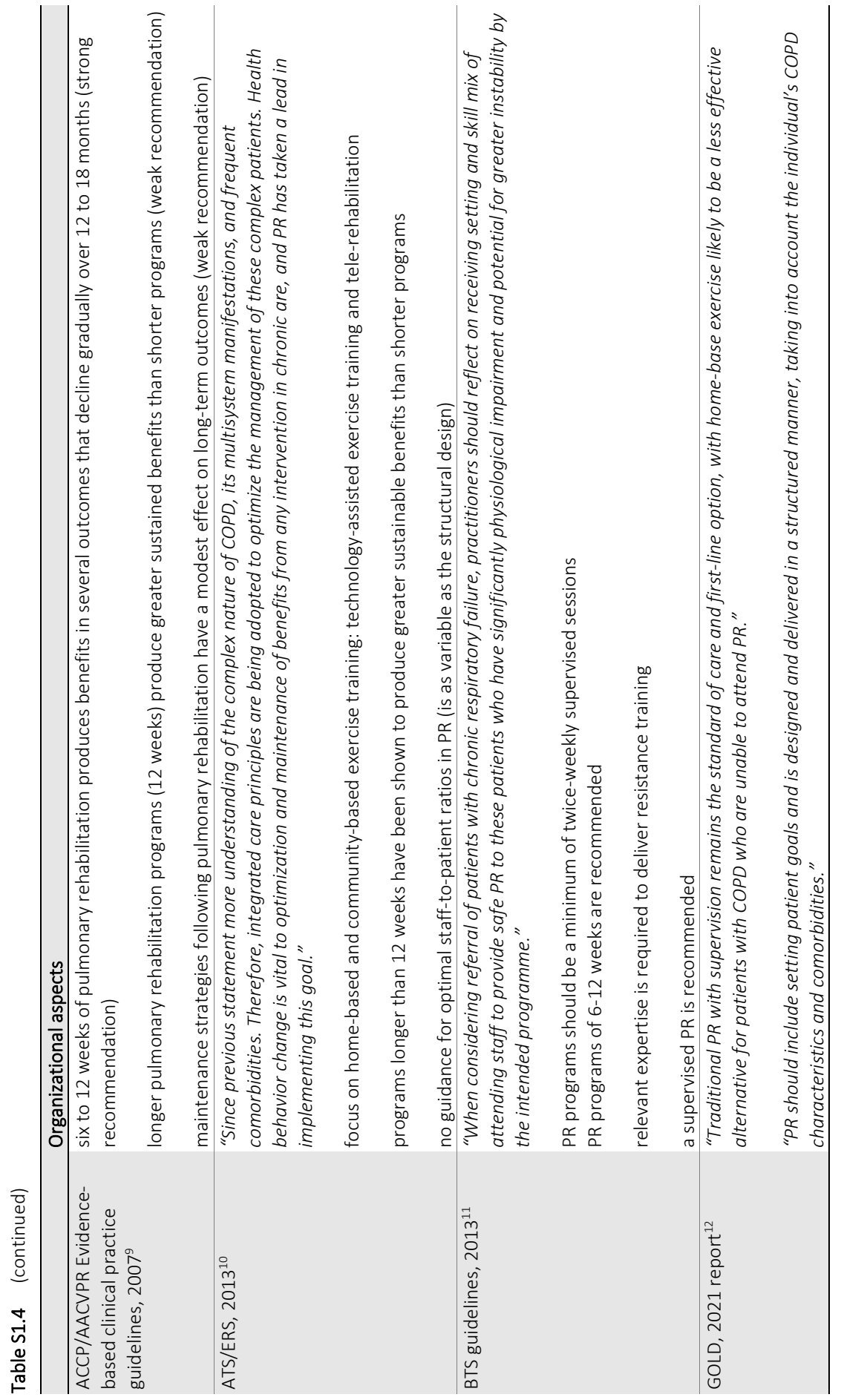




\section{Supplemental references}

1. Hodgkin JE, et al. American Thoracic Society. Medical Section of the American Lung Association. Pulmonary rehabilitation. Am Rev Respir Dis. 1981;124(5):663-6.

2. Donner CF, Howard P. Pulmonary rehabilitation in chronic obstructive pulmonary disease (COPD) with recommendations for its use. Report of the European Respiratory Society Rehabilitation and Chronic Care Scientific Group (S.E.P.C.R. Rehabilitation Working Group). Eur Respir J. 1992;5(2):266-75.

3. Fishman AP. Pulmonary rehabilitation research. Am J Respir Crit Care Med. 1994;149(3 Pt 1):825-33.

4. Donner CF, Muir JF. Selection criteria and programmes for pulmonary rehabilitation in COPD patients. Rehabilitation and Chronic Care Scientific Group of the European Respiratory Society. Eur Respir J. 1997;10(3):744-57.

5. Pulmonary rehabilitation: joint ACCP/AACVPR evidence-based guidelines. ACCP/AACVPR Pulmonary Rehabilitation Guidelines Panel. American College of Chest Physicians. American Association of Cardiovascular and Pulmonary Rehabilitation. Chest. 1997;112(5):1363-96.

6. Britisch Thoracic Society (BTS), British Thoracic Society guidelines for the management of chronic obstructive pulmonary disease. The COPD Guidelines Group of the Standards of Care Committee of the BTS. Thorax. 1997;52 Suppl 5:S1-28.

7. American Thoracic Society (ATS), Official Statement of the American Thoracic Society. Pulmonary Rehabilitation - 1999. Am J Respir Crit Care Med. 1999;159:1666-82.

8. Nici L, et al. American Thoracic Society/European Respiratory Society statement on pulmonary rehabilitation. Am J Respir Crit Care Med. 2006;173(12):1390-413.

9. Ries AL. ACCP/AACVPR evidence-based guidelines for pulmonary rehabilitation. Round 3: another step forward. J Cardiopulm Rehabil Prev. 2007;27(4):233-6.

10. Spruit MA, et al. An official American Thoracic Society/European Respiratory Society statement: key concepts and advances in pulmonary rehabilitation. Am J Respir Crit Care Med. 2013;188(8):e13-64.

11. Bolton CE, et al. British Thoracic Society guideline on pulmonary rehabilitation in adults. Thorax. 2013; 68 Suppl 2:ii1-30.

12. Global Initiative for Chronic Obstructive Lung Disease (GOLD). Global Strategy for Prevention, Diagnosis and management of COPD. 2021.

13. Tiep BL. Pulmonary Rehabilitation Program Organization. Principles and Practice of Pulmonary Rehabilitation, ed. R. Casaburi and T.L. Petty. 1993, Philadelphia: Saunders. 



\section{Chapter 2}

Aim and outline of the thesis 



\section{Aim and outline of the thesis}

Chapter 1 describes the fast evolving field of chronic respiratory diseases in general and COPD in particular. This chapter outlines the concepts on pulmonary rehabilitation as formulated in appealing definitions during the previous decades. Chapter 1 focuses attention on the growing reliance on evidence based medicine concepts, making it a daily challenge to argue value-based health care particularly for these patients referred for their high needs and high burden of disease. As discussed in Chapter 1, organizational and process characteristics of pulmonary rehabilitation are poorly defined making bench marking between programs and settings very difficult or even impossible. Although definitions of pulmonary rehabilitation claim to address all limitations and needs experienced by the patient, there seems a huge chasm between these theoretical concepts and daily practice. As such, pulmonary rehabilitation is an important intervention that is often not delivered, and pulmonary rehabilitation programs that are delivered are often not addressing real needs.

This thesis aims to develop an understanding of the possible interactions between the number of pulmonary and extra-pulmonary components in COPD patients admitted for pulmonary rehabilitation and to assess the spectrum of heterogeneity of these components based on real life data integration. Furthermore, performance of an integrated, individualized intervention program will be evaluated in a sample of patients with COPD admitted to the pulmonary rehabilitation center Ciro as well in traits-based subgroups.

Starting point of this PhD-thesis was the development of an organizational model of a patient tailored program based on a standardized assessment and systematic outcome evaluation at the end of the intervention. Based on the initial assessment a modular patient tailored program is offered to overcome these needs. This patient tailored program formed the basis for standardization of pulmonary rehabilitation between the Dutch Lung Centers. This model is described in Chapter 3. By transfer of all these real life data anonymously to an integrated knowledge system (see Figure 2.1), we aimed to better identify the right patient, for the right treatment with the right outcomes. Therefore, we have chosen to perform all statistical analyses in chapters 4, 5, 6, 7 and 8 using self-organizing maps by Viscovery SOMine 7.3 build 7427 by Viscovery Software $\mathrm{GmbH}$ (www.viscovery.net; Vienna, Austria). Self-organizing maps (SOMs, also referred to as Kohonen maps) are used to create an ordered representation of selected attributes. The SOM method can be viewed as a non-parametric regression technique that simplifies complexity by converting multidimensional data spaces into lower dimensional abstractions. A SOM generates a non-linear representation of the data distribution and allows the user to identify homogeneous data groups visually to reveal meaningful relationships. 


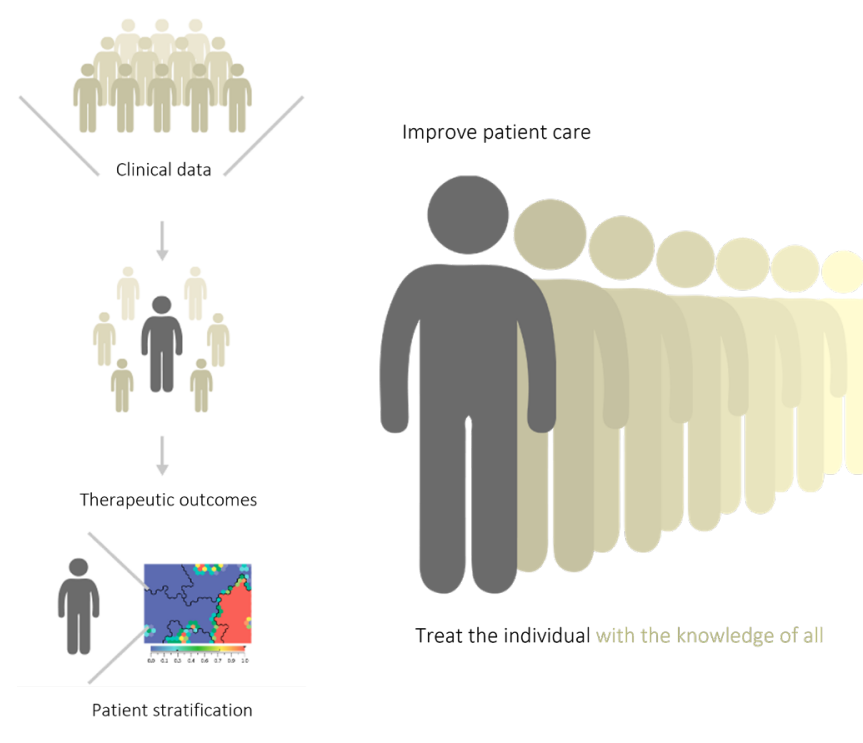

Figure 2.1 Improvement of patient care.

The integrated approach described in this thesis could be considered as a model, applicable to offer personalized, individualized care to patients with high needs related to other chronic non-communicable diseases. Following research questions are addressed in this thesis:

- Is it possible to identify clusters in COPD based on a comprehensive lung function assessment only? These data are described in Chapter 4.

- Is it possible to profile a multidimensional response to pulmonary rehabilitation? These data are described in Chapter 5.

- To what extent a physiomic clustering predicts outcomes of pulmonary rehabilitation? (Chapter 6)

- Is clustering based on pulmonary and extra-pulmonary traits more accurate to identify patient subgroups? (Chapter 7)

- Is an integrated assessment an indicator of multidimensional outcomes after PR? (Chapter 8). 
The results of these and other studies and lessons learned are discussed in Chapter 9. Chapter 10 offers future perspectives for management of the growing burden of high costs, high needs patients in our society. 



\title{
Chapter 3
}

\begin{abstract}
Understanding complexity of chronic noncommunicable diseases: an integrated approach for personalized management of patients with COPD
\end{abstract}

Ingrid ML Augustin, Martijn A Spruit, Frits ME Franssen and Emiel FM Wouters Clin Res Pulmonol 2015;3(2):1034. 


\section{Abstract}

Non-communicable diseases (NCDs) will increase over the next decades. Understanding the complexity of chronic NCDs and the adaptation of the health care system to implement new management strategies addressing the patients' needs, are still major challenges. Despite all the efforts aligning health care delivery with their needs, patients with chronic NCDs are still confronted with fragmented, complex health care systems. Health care management of NCDs needs a better understanding of the complexity of the disease in order to offer and organize more effective therapies to reduce the huge societal and economic burden of these diseases. In this paper, a patient-centered, personalized health care organizational structure for COPD patients with a high disease burden is presented. This could serve as a model for the management of chronic and complex NCDs in general. 


\section{Introduction}

Cardiovascular diseases, cancers, chronic respiratory diseases and diabetes are the four major chronic non-communicable diseases (NCDs). Together, they represent a major global health burden. Indeed, these NCDs are responsible for 36 million annual deaths or $63 \%$ of the total number of deaths. ${ }^{1}$ The total number of annual NCD-related deaths is projected to increase up to 55 million by 2030 due to the growth of population and the increased longevity. ${ }^{2}$ To a large extent, NCDs develop as a result of an unhealthy lifestyle, such as tobacco use, physical inactivity, unhealthy diet and/or the excessive use of alcohol. ${ }^{1}$ These lifestyle conditions, at least in part, seem related to a lower socioeconomic status. ${ }^{1}$ The risk factors for the major NCDs are still increasing worldwide, and even a general pattern of health style improvement will only result in positive effects decades from now. ${ }^{3}$ Therefore, the World Health Organization (WHO) global strategy not only focuses on surveillance and prevention, but considers health care management as the third key component of an integrated approach to tackle NCDs. ${ }^{4}$

One of the biggest challenges in health care management is to understand the growing complexity of these chronic NCDs. Besides understanding the complexity of geneenvironment interactions, NCDs manifest in different phenotypic appearances during the disease history as a consequence of irreversibility of pathophysiological changes and the absence of disease-modifying interventions. ${ }^{5}$ Current health care ignores this heterogeneity in the burden of NCDs and largely fails to offer a personalized, patientcentered approach. Furthermore, personalized interventions are generally evaluated on direct medical costs, thereby following/adhering to the usual approach in acute medical interventions, but ignoring the lifespan impact of patient-centered and demand-driven disease management. In this opinion paper, future management strategies for patients with Chronic Obstructive Pulmonary Disease (COPD) are discussed as a possible model of management of complex chronic NCDs in general.

\section{The burden of COPD}

COPD, a common preventable and treatable disease, is characterized by persistent airflow limitation that is usually progressive and associated with an enhanced chronic inflammatory response in the airways and lungs to noxious particles or gases. ${ }^{6}$ Intriguingly, the Burden of Obstructive Lung Disease (BOLD) program shows a substantial prevalence of COPD among never-smokers (3-11\%). ${ }^{7}$ This may be due to occupational and environmental exposures, lifestyle and/or genetic factors. ${ }^{8}$

In European cities, 5-10\% of adults aged over 40 years has COPD, with a higher prevalence in men than in women. ${ }^{8}$ In people aged $>70$ years, the prevalence of COPD is about $20 \%$ in men and $15 \%$ in women. ${ }^{8}$ Overall, COPD mortality rate for men and 
women in Europe is about 18 per 100000 inhabitants per year. ${ }^{8}$ COPD is associated with a significant economic burden. In the European Union, the total direct costs for respiratory diseases are estimated to be about $6 \%$ of the total health care budget, with COPD accounting for $56 \%$ ( $€ 38.6$ billion) of these costs. ${ }^{8}$

In the United States, COPD is the third leading cause of death behind cancer and heart disease, with an age-adjusted death rate of 41.2 per 100000 population in $2009 .^{9}$ The American Lung Association shows an aged-adjusted prevalence for adults of $5.2 \%$ for men and $7.2 \%$ for women. ${ }^{9}$ Data taken from the BOLD project demonstrate for the United States a prevalence of about $12.7 \%$ for men and $15.6 \%$ for women aged over 40 years. ${ }^{7,10}$ In people aged $>70$ years, $19.2 \%$ of men and $29.6 \%$ of women have COPD. ${ }^{7}$ In the United States, the annual costs for COPD in 2010 were $\$ 49.9$ billion. This includes $\$$ 29.5 billion in direct health care expenditures, $\$ 8.0$ billion in indirect morbidity costs and $\$ 12.4$ billion in indirect mortality costs. ${ }^{9}$

In 1990, COPD was the twelfth leading cause of Disability-Adjusted Life Years (DALYs) lost in the world, responsible for $2,1 \%$ of the total. According to the projections, COPD will be the seventh leading cause of DALYs loss worldwide in 2030. ${ }^{11}$ Indeed, COPD will become the seventh largest disease burden and the fourth greatest cause of death by $2030 .{ }^{12}$

\section{Improving the management of COPD: towards disease phenotyping}

It is widely recognized that COPD is a complex syndrome with numerous pulmonary and extra-pulmonary components. ${ }^{13}$ COPD, diagnosed by assessment of the degree of airflow limitation, is nowadays considered as identification of the COPD syndrome without offering any information about disease burden or complexity. ${ }^{13}$ Significant heterogeneity exists with respect to clinical presentation, physiology, imaging, response to therapy, decline in lung function, and survival amongst patients with COPD, irrespective of the degree of airflow limitation. ${ }^{13}$ Exacerbations and comorbidities contribute to the overall disease severity in individual patients. ${ }^{6}$

The global Strategy for the Diagnosis, Management and Prevention of COPD (GOLD) has proposed a three-domain assessment of COPD (Figure 3.1), which, besides the severity of airflow limitation, also includes the level of symptoms experienced by the patient and the previous history of exacerbations and hospital admissions. ${ }^{6}$ 


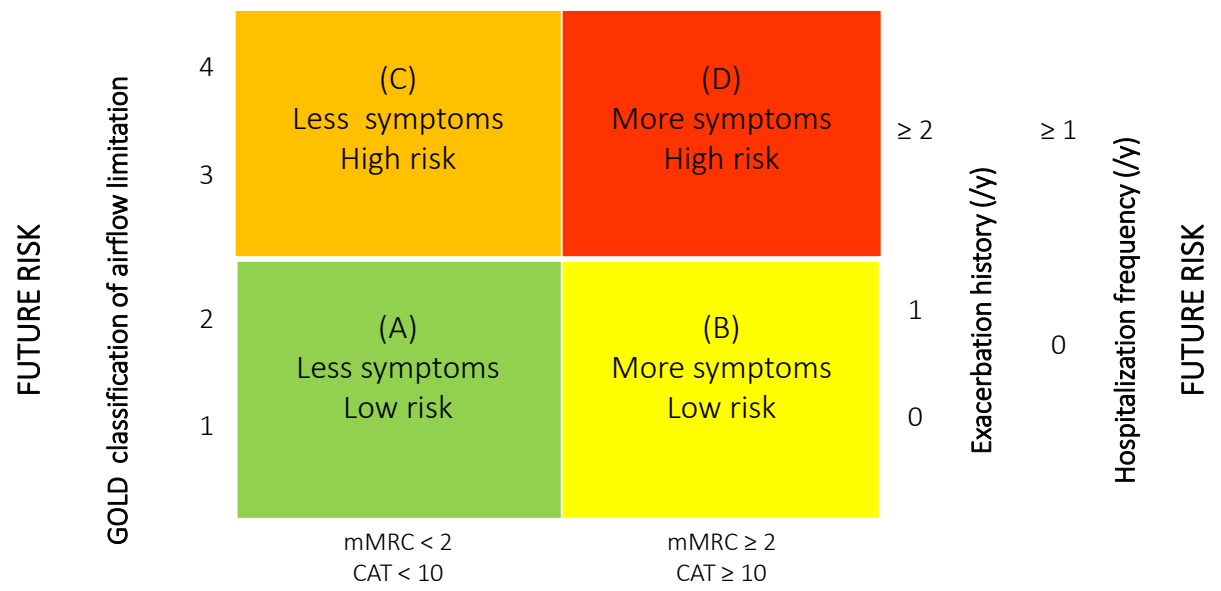

CURRENT SYMPTOMS

Figure 3.1 Combined GOLD assessment of COPD.

Patients are stratified in 4 groups (A, B, C or D) based on these parameters. This approach reflects a pragmatic, expert-based patient stratification, which requires prospective validation in a wide variety of patients with COPD. ${ }^{6}$ The GOLD assessment scheme largely aims to support formulation of pharmacological therapies in the different groups of patients with COPD, but offers no tools to assess the individual disease burden in order to set-up appropriate management strategies, including non-pharmacological interventions and diagnosis/treatment of comorbidities. Indeed, exercise capacity, lower-limb muscle function, health status, problematic activities of daily life, and objectified comorbidities varied to a great extent in a sample of patients with COPD consisting only of GOLD group D. ${ }^{14}$

To date, it is widely recognized that identification and subsequent grouping of key attributes of COPD into clinically meaningful and useful subgroups or phenotypes is needed in order to guide more effective therapies and management strategies. A COPD phenotype should be able to classify patients into distinct subgroups that provide prognostic information and allow more appropriate therapy that alters clinically meaningful outcomes. ${ }^{13}$ This concept of clinical COPD phenotypes is based on the description of differences between individuals with COPD by a single or a combination of disease attributes. ${ }^{13}$ Ideally, assessment of the complexity of COPD needs to include, not only the degree of impairment in the diseased organ, but also the extra-pulmonary components, comorbidities, and environmental factors and their impact on the individual patient. For example, five clusters of comorbidities were identified in patients with COPD entering pulmonary rehabilitation: a cluster with less comorbidity, a 
cardiovascular cluster, a cachectic cluster, a metabolic cluster, and a psychological cluster. ${ }^{15}$ These data emphasize that comorbidities co-occur in patients with COPD. Moreover, the psychological cluster stresses the importance to include the psychological and emotional impact of COPD. The assessment and management of patients with COPD will have to consider this disease heterogeneity in order to provide the best possible care to individual patients. Phenotyping of COPD patients linked with clinically relevant outcome parameters and medical consumption criteria offers perspectives for better and more efficient health care management.

\section{Towards individualized COPD management}

Despite its limitations ${ }^{16}$, applying GOLD's three-domain assessment system illustrates in part the heterogeneity in disease burden in different samples of patients with COPD. Depending on the sample studied, the prevalence of the different GOLD categories varies. In a sample from the general population, group $A$ is the most prevalent (77\%). ${ }^{17}$ On the one hand, in patients with COPD treated in primary, secondary and/or tertiary care settings, the proportion of GOLD group A seems clearly lower (about one-third). ${ }^{18-22}$ On the other hand, about one-third of the patients with COPD in primary/secondary/ tertiary care settings is identified by severe airflow limitation, high symptom scores and high-risk profile. ${ }^{18-22}$ Although to date limited data are available on the stability of the GOLD classification over time, these GOLD categories seem relatively stable over time. ${ }^{19}$ These studies not only reflect the heterogeneity of COPD itself, but also the enormous spread in disease burden irrespective of the levels in care organization. As the current GOLD classification largely focuses on pharmacological therapy of COPD, the possibilities of this classification for the organization of a demand-driven integrated health care management still remain unexplored. Future studies are needed to explore this option and to validate the currently applied markers as reflective for the experienced individual burden of COPD.

\section{The current health care organization for chronic conditions}

Current GOLD disease management strategies clearly reflect the persistent emphasis on diagnosis, thereby ruling out other serious diseases and symptom-relieving treatments. They rely on patient-initiated visits, relief of symptoms, normalization of lung function, assurance that there is no urgent medical crisis, and on prevention and treatment of acute or chronic emergencies as exacerbations. In this medically oriented approach, clinical judgment is directed to achieve diagnostic and therapeutic certainty, based on reductionist thinking to break down the clinical problem in circumscribed domains or parameters. ${ }^{23,24}$ Such an approach only partly fits with patient-centered goals of chronic 
care: enhancement of functional status, minimization of distressing symptoms, enhancement of quality of life, and prolongation of life. ${ }^{25}$ Nevertheless, many factors influencing the quality of health care are largely overlooked: psychosocial distress often remains undetected or inadequately managed; whereas insufficient attention for education, relevant skills, motivation, and feedback will lead to failures in selfmanagement of the disease or risk factors as a result of patient non-engagement and/or ignorance. ${ }^{26}$ Particularly in the management of patients suffering from chronic diseases, it is very important to view a human being as composed of and operating within multiple interacting and self-adjusting systems, including biochemical, cellular, physiological, psychological and social systems. Illness arises from the dynamic interaction within and between these systems, and not from a failure of a single component as chronic airflow limitation in COPD. ${ }^{23}$

Current guidelines focus on traditional characteristics of the disease condition itself. They ignore the need to apply a more holistic approach for the individual patient and the need to create an approach of the individual as a complex adaptive system (CAS), thus implying diversity consisting of a wide variety of elements (complex) and implying the capacity to change or the ability to learn from experience (adaptive). ${ }^{27}$ To overcome diagnostic and therapeutic uncertainty, the premise of most guidelines is to focus on evidence-based medicine, defined as the conscientious, explicit and judicious use of current best evidence in making decisions about individual patient care. ${ }^{28,29}$ More specifically, evidence-based medicine is defined as the use of mathematical estimates of the risk of benefit and harm, derived from high-quality research on population samples, to inform clinical decision-making in the diagnosis, investigation or management of individual patients. $^{30}$ Evidence-based medicine relies therefore on predictable, quantitative research, especially from randomized clinical trials (RCTs). Many studies have criticized the extrapolation of these outcomes to real-life conditions, based on the huge selection of patients to become part of these trials. Particularly since primary care COPD patients stand out from patients enrolled in large RCTs in terms of gender, lung function, quality of life and exacerbations. . $^{31,32}$

However, clinical judgment involves an irreducible element of factual uncertainty and relies to a greater or lesser extent on the interpretation of the illness' wider history. ${ }^{30}$ Maintenance or re-establishment of health can be achieved through a holistic approach of the illness. ${ }^{23}$ Effective clinical decision-making in such a complex system must accept unpredictability and is built on subtle emergent forces within the overall system: a small change in one part of the network of interacting systems may lead to a much larger change in another part through amplification effects. ${ }^{23}$

The effectiveness of such interventions is highly dependent on the context in which health care is delivered. ${ }^{24}$ Besides the complexity of the illness, the complexity of health care is largely neglected in daily practice. Many factors interact in health care, including 
patient factors (e.g., personal, cultural, socioeconomic), factors related to the health care professionals (e.g., training, expertise, interests), task-related factors (e.g., the particular health care task, workflow, available time and technology), team-related factors (e.g., communication, roles, leadership), environmental factors (e.g., physical, social and pollution), and organizational factors (e.g., organizational structure, culture, policies and procedures). ${ }^{33}$

Although the interest in organizational contributions to the delivery of care has risen significantly in recent years, coordination of medical resources for patients across the entire delivery system is still a tremendous challenge. ${ }^{34}$ Despite recognition of the importance of health care organizations and growing research on the relationship between organizational aspects and quality of health care, no clear conclusions have emerged from the literature. ${ }^{35}$ In current health services research, theory plays a minor role and methodological approaches are mainly focused on cross-sectional, quantitative designs. Another methodological shortcoming of research is the restricted attention to a single organizational level, thereby failing to take into consideration the nested structure of health care organizations and the consequences of such nesting for quality of care. ${ }^{35}$ Theories, methodologies and data are needed to link all the three components of structure, process and outcome together, instead of looking for structure-outcome, structure-process and/or process-outcome relationships. ${ }^{35}$

In 2001, the Institute of Medicine (IOM) already identified that patients and their families must try to navigate in a fragmented, complex health care system with insufficient information and an unclear understanding of how to find the best-quality care for their specific needs and wishes. Similarly, health care professionals face pressures to improve quality and measurable outcomes without having systems in place that can help them to identify best practices or means of arranging follow-up for a patient's need across the entire continuum of care. Purchasers largely lack adequate outcomes and/or process characteristics for benchmarking health care delivery systems. $^{36}$

In conclusion, current management of chronic conditions such as COPD is still largely based on a reductionist thinking and Newton's 'clockwork universe' metaphor for solving clinical and organizational problems. ${ }^{24}$ To cope more adequately with the escalating burden of chronic disease conditions, health care must respond flexibly to emerging patterns and opportunities. ${ }^{24}$

\section{Heterogeneity of COPD and organization of care}

At least in certain subgroups, COPD is a complex medical problem, with dynamic, nonlinear interactions between different disease components along time. Heterogeneity 
indicates that different disease components are present in these patients at different time points of their medical history. This disease heterogeneity is now largely ignored, describing all the efforts pursued in many ways and in different health systems to realize integration and/or coordination of care. ${ }^{37}$ The outcome is an academic quagmire of definitions and concept analyses surrounding the notion of integration. ${ }^{38}$ The wide GOLD stage distribution in patients with COPD managed in primary and secondary care reflects the ignorance of individual burden of needs by the patient in allocation of health care services. Many COPD disease management programs estimating and organizing patients with COPD in echelons and services are still based on a traditional, pathophysiological disease perspective, focused mainly on the degree of airflow limitation. ${ }^{39-41}$

Already in 1999, Leutz proposed an integration framework for chronic conditions, enabling a comprehensive approach, which responds to the varied needs of persons with chronic and/or disabling conditions. In this approach, dimensions of need are defined in terms of stability and severity of the patient's conditions, duration of illness, urgency of the intervention, scope of services required, and the user's capacity for selfdirection. ${ }^{42}$ Following this line of reasoning, Leutz divided service users into three groups: those with mild-to-moderate but stable conditions, those with moderate levels of need, and those with long-term, severe, unstable conditions who frequently require urgent interventions and who have limited capacity for self-direction. ${ }^{42}$ Particularly, the latter group will benefit from a high level of integration of the different service domains operating as multidisciplinary teams. ${ }^{42}$ Bodenheimer and colleagues applied these concepts in a population management model and divided patients with chronic conditions into three distinct groups based on their degree of need. ${ }^{43}$ Patients at level 1 have a relatively low level of health care needs: their chronic condition is reasonably under control, with support for self-management of their chronic condition provided through a primary care team. Level 2 patients are considered at increased risk because their condition is unstable or because they can deteriorate, unless they have structured support through specialist management. Finally, level 3 persons include individuals with highly complex needs and/or high intensity of unplanned secondary care: these persons require active management through case managers. ${ }^{43}$ Therefore, the application of a stratified, population-based care model can create a much more efficient, patientdirected care management approach and offer an appropriate response to the exponentially increasing economical and societal burden of chronic diseases as COPD. ${ }^{3,8,11,44}$ Stratifications of diseased populations as proposed by Leutz $^{42}$ and Bodenheimer and colleagues ${ }^{43}$ have never been done in patients with COPD. GOLD's COPD classification system is a first step towards that direction, but clearly needs further sophistication, validation, and implementation in order to improve quality of COPD care. Notwithstanding, it remains a great step forward compared to earlier GOLD classifications, which were solely based on the degree of airflow limitation. 


\section{Pulmonary rehabilitation: targeting complex needs}

A patient-centered approach of patients with chronic respiratory diseases such as COPD is not new in pulmonary medicine. Indeed, the first authoritative statement of pulmonary rehabilitation from the American College of Chest Physicians, published in 1974, introduced pulmonary rehabilitation already as an art of medical practice, wherein an individually tailored, multidisciplinary program was formulated. Through accurate diagnosis, therapy, emotional support and education, this program stabilizes or reverses both physiopathological and psychopathological manifestations of pulmonary diseases. Also, it attempts to return the patient to the highest possible functional capacity allowed by the handicap and overall life situation. ${ }^{45}$ In 1994, the National Institutes of Health defined pulmonary rehabilitation as a multidimensional continuum of services for the patient and the family supplied by an integrated team of specialists in complementary disciplines, with the goal of the patient living and functioning independent within society. ${ }^{46}$

The 2013 ATS/ERS statement defined pulmonary rehabilitation as a comprehensive intervention based on thorough assessment followed by patient-tailored therapies designed to improve the physical and psychological condition of people with chronic respiratory disease and to promote the long-term adherence to health-enhancing behaviors. ${ }^{47}$ The latter part of the definition fits with the definition provided in the addendum in the American Association for Respiratory Care stipulating that pulmonary rehabilitation should be both restorative and preventive. ${ }^{48}$

The main, common points among the various definitions of pulmonary rehabilitation include (1) a focus on chronic respiratory patients and their caregivers; (2) an individualization of the intervention; (3) an ongoing multidisciplinary intervention; (4) outcomes based on physiological, psychological and social measures considering a global dimension to the individual's health; and (5) the stimulation of long-term adherence to health-enhancing behavior(s) in order to promote autonomy and social participation of the patient. The diagnosis of physiopathological and psychopathological problems in the individual patient forms the start of every pulmonary rehabilitation program as illustrated in Figure 3.2. 


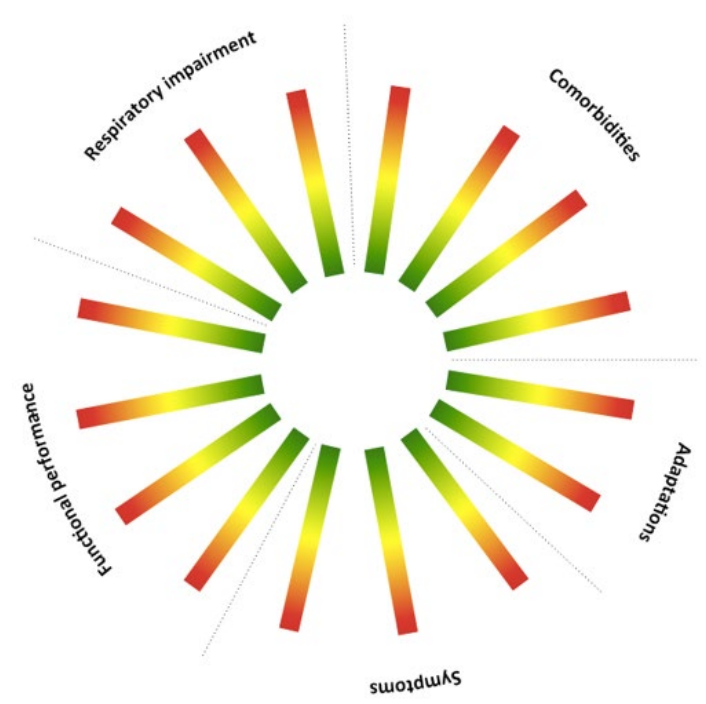

Figure 3.2 The different domains of an integrated assessment: determining the complexity of the disease is not the result of one measurement within one domain, but the combination of the measurements at risk (yellow) or impaired (red) of all domains. It means that one measurement in one of the domains showing no problem (green) is not illustrative for the degree of complexity of the disease.

More recently, control panels for personalized medicine of chronic diseases as COPD were suggested: in fact, these so-called control panels are not new and already applied for more than half of a century in dedicated pulmonary rehabilitation settings. ${ }^{49}$ Pulmonary rehabilitation is therefore more than a path to personalized medicine in COPD. It really offers personalized medicine in clinical practice. ${ }^{50}$ Assessment of available pulmonary rehabilitation services stresses the need for evaluation of the psychopathological impact of the disease condition. ${ }^{47}$

Although definitions of pulmonary rehabilitation are widely accepted, huge variability exists in content and organizational aspects among pulmonary rehabilitation programs, largely the result of local conditions and financial resources. ${ }^{51} \mathrm{~A}$ recent international survey clearly illustrates the large differences among pulmonary rehabilitation program across continents, including the composition of the rehabilitation teams. ${ }^{51}$ The survey also illustrates that most programs are small-scale interventions (median 40 to 75 enrolled individuals per program per year), and that most teams consisted of a median of 5 health care professionals: chest physicians, dieticians, nurses and physiotherapists were the most prevalent team members. ${ }^{51}$ The individualization of the goals of pulmonary rehabilitation is not reflected in the selection of the three most important outcomes as identified by health care professionals: health status, dyspnea, and exercise capacity. ${ }^{51}$ Even mono-disciplinary and strictly educational programs are still described 
as pulmonary rehabilitation programs, completely neglecting the individualized assessment and multidisciplinary intervention. ${ }^{41,52,53}$ Furthermore, the absence of real performance and process metrics hampers meaningful comparisons and benchmarking among programs in different jurisdictions, and does not allow quality control to ensure appropriate standards for pulmonary rehabilitation.

\section{The process of pulmonary rehabilitation: COPD management beyond the control panel}

Pulmonary rehabilitation advocates a personalized approach and aims for patients with health care professionals, more pro-active in the daily management of their disease. This process of health care organization around the patient, i.e. to adopt a patient-centered approach, is generally underestimated or neglected in the management of patients with chronic conditions as COPD. ${ }^{54}$

Ideally, COPD management must offer a flexible, holistic, and integrated intervention, based on partnering of different skills to achieve shared, individualized, patient-related objectives, and to achieve improvement in clinically relevant outcomes and added value to the patient and the community. This requires a process-based organization to manage business around these core processes (e.g., intake and assessment, rehabilitative therapies, and outcome evaluation). ${ }^{55}$

The sociotechnical systems theory has been developed to design and change organizations in relation to the environmental conditions and strategic choices, and to address the increasing complexity of organizations as a result of increasing external uncertainty and variation within the internal division of labor, as required to offer a tailor-made, individualized program. ${ }^{56}$ The sociotechnical theory offers a framework to improve efficiency, quality, flexibility and innovation. ${ }^{56}$ Indeed, a key feature of sociotechnical design involves bringing together people from different roles and disciplinary backgrounds who have different skills, experience and expertise. Pluralism is the norm, and this implies that they share their views and expertise. They need to educate one another in the opportunities that may exist for the design of a new system, and what they have to offer the design process. ${ }^{57}$ Actually, the sociotechnical theory offers a framework for health care organizations to create value by improving outcomes that matter most to patients relative to the costs of achieving those outcomes. ${ }^{58}$ The holistic approach of the process, like pulmonary rehabilitation, means organizing around the customer and the need: it has the features of an integrated practice unit (IPU) that treats not only a disease but also the related conditions, complications, and circumstances that commonly occur along with it. In an IPU, personnel regularly work together as a team towards the common goal of maximizing the patient's overall outcomes as efficiently as possible. Actually, organizing pulmonary rehabilitation 
according to the sociotechnical principles meets the features of a high-value health care organization. ${ }^{59}$

Figure 3.3 illustrates an example of a patient-tailored program, composed by different modules. Each module exists of different therapies in order to achieve the goal of the specific module, which consequently contributes to the medical outcome and the patients' overall goal of treatment. The basic modules can be supplemented by specific burden-assessment-driven modules. This modular approach makes it possible to individualize the treatment. Figure 3.4 depicts the integrated baseline assessment, by which the degree of the complexity of COPD is determined, and in turn it depicts the treatment program with its specific modules. Depending on the individual needs and wishes of the COPD patient, each program will differ.

Pulmonary rehabilitation can offer a holistic approach by considering patients as complex adaptive systems: a modular program structure does not mean that illness and patient behavior is modeled as a simple cause and effect system. ${ }^{60} \mathrm{~A}$ patient-centered, demand-driven rehabilitation program aims to seek concordance with the patient. ${ }^{61}$ Most patients referred for pulmonary rehabilitation experience a large tension to change: in such circumstances, a small influence can have a large effect in behavior and outcomes. ${ }^{23,62,63}$ Furthermore, the effectiveness of interventions such as pulmonary rehabilitation will be highly dependent on the context in which the program is delivered. ${ }^{23,24}$ Therefore, all health care professionals need to partner with the patient and work closely with other providers to improve the outcomes. ${ }^{54}$

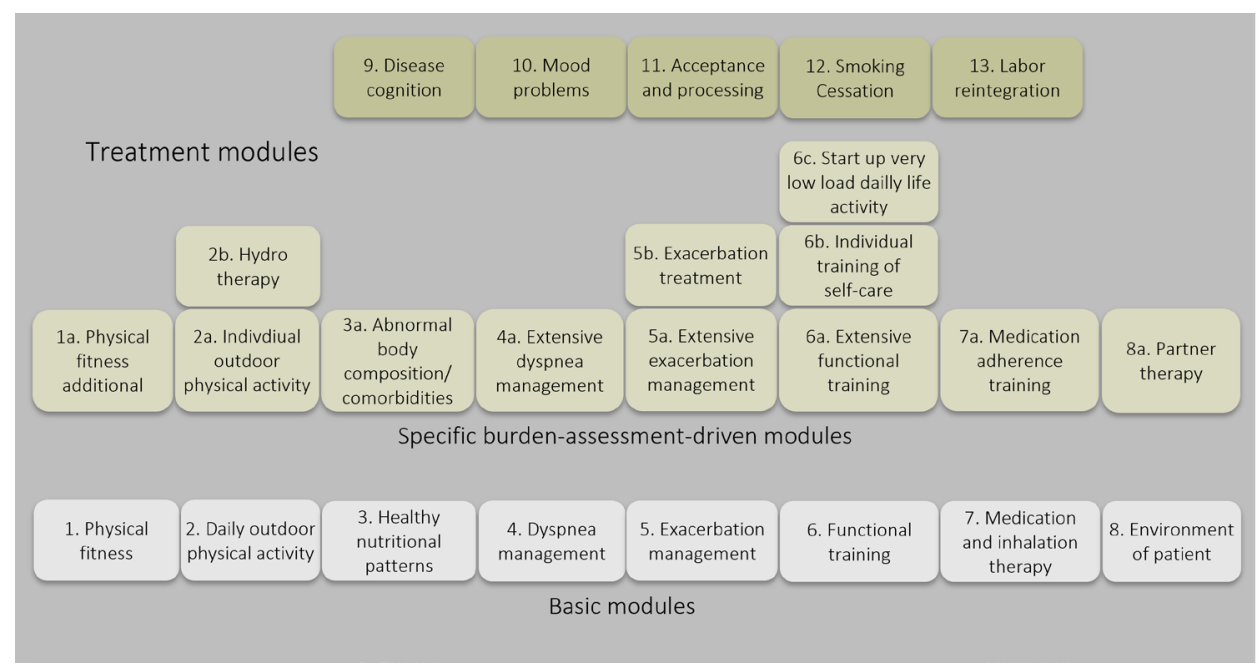

Figure 3.3 Basic and specific burden driven treatment modules for composing a patient tailored program. 


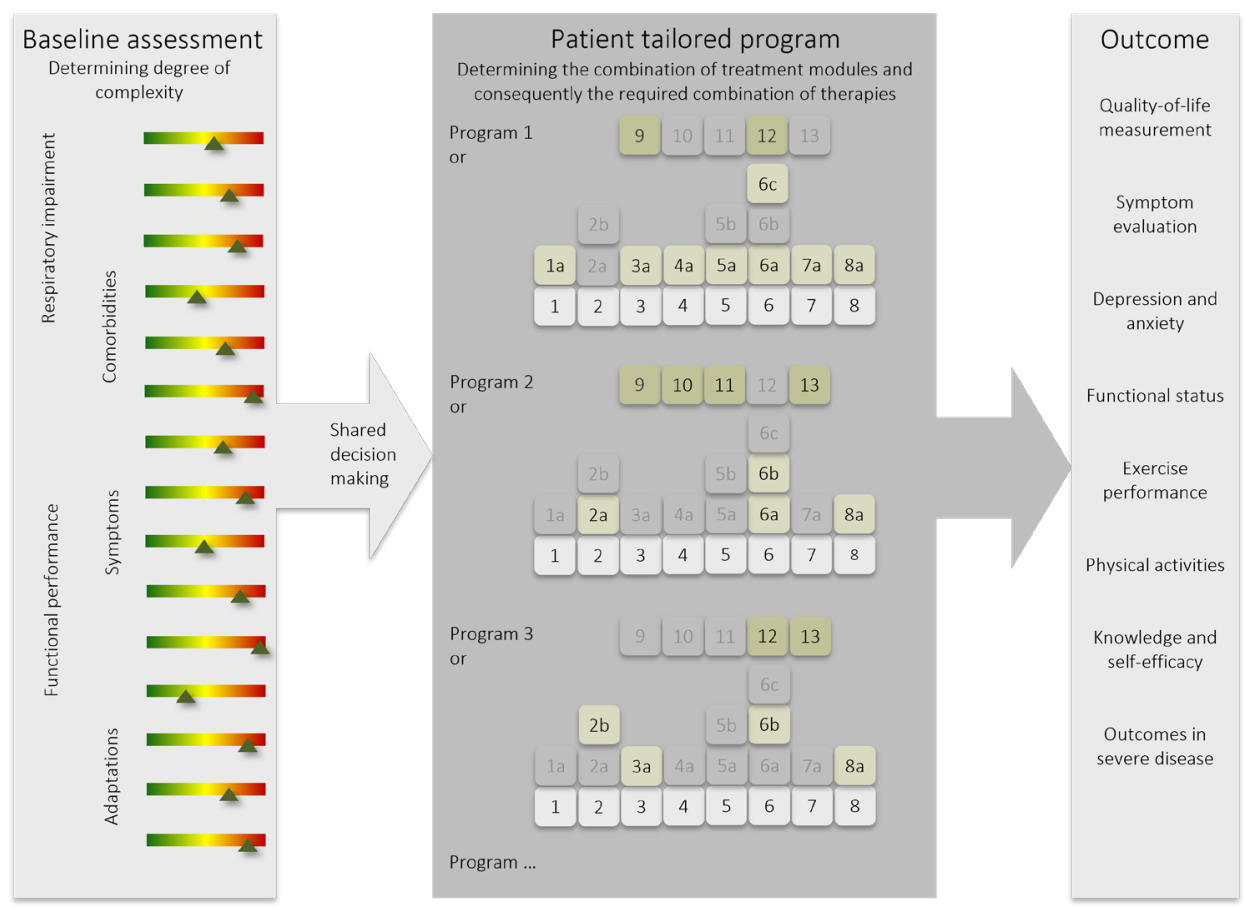

Figure 3.4 The process of a patient tailored program: after an integrated baseline assessment, by which the degree of the complexity of COPD is determined, a treatment program is composed of at least all the basic modules. Depending on the individual needs and wishes of the COPD patient, specific burden driven modules can be added. Each individualized program is followed by an outcome measurement of the different domains as described.

\section{Conclusions}

Besides the quantitative burden of chronic NCDs worldwide, the complexity of medicine and health care has increased tremendously. The traditional "clockwork universe", in which big problems can be broken down into smaller ones, analyzed and solved by rational deduction, still strongly influences the practice of medicine. However, human beings are composed of and operating within multiple interacting and self-adjusting systems, and illness arises from the dynamic interaction within and between these systems. The science of complex adaptive systems will provide important concepts and tools for responding to the current challenges in health care. ${ }^{24}$ Concepts of complex adaptive systems are described for COPD as a model for chronic illness conditions. Pulmonary rehabilitation offers a model for such a holistic approach. 


\section{References}

1. World Health Organization (WHO), Global status report on noncommunicable diseases 2010. WHO, Geneva, Switzerland, 2011.

2. World Health Organization (WHO), World Health Statistics 2012. WHO, Geneva, Switzerland, 2012.

3. World Economic Forum, The Global Economic Burden of Non-Communicable Diseases. The World Economic Forum and Harvard School of Public Health, 2011.

4. World Health Organization (WHO), Framework for action to implement the United Nations Political, Declaration on Noncommunicable Diseases. WHO, Geneva, Switzerland, 2011.

5. Bousquet J, et al. Systems medicine and integrated care to combat chronic noncommunicable diseases. Genome Med. 2011;3(7):43.

6. Global Initiative for Chronic Obstructive Lung Disease (GOLD), I., Global Strategy for the Diagnosis, Management and Prevention of Chronic Obstructive Pulmonary Disease.. http://www.goldcopd.org, 2014.

7. Buist AS, et al. International variation in the prevalence of COPD (the BOLD Study): a population-based prevalence study. Lancet 2007;370(9589):741-50.

8. European Respiratory Society (ERS), European Lung White Book: Huddersfield. European Respiratory Society Journals, 2013.

9. http://www.lung.org/finding-cures/our-research/trend-reports/copd-trend-report.pdf.

10. Mannino DM, Buist AS. Global burden of COPD: risk factors, prevalence, and future trends. Lancet. 2007;370(9589):765-73.

11. Mathers CD, Loncar D., Projections of global mortality and burden of disease from 2002 to 2030. PLoS Med. 2006;3(11):e442.

12. World Health Organization (WHO), Global surveillance, prevention and control of chronic respiratory diseases : a comprehensive approach. WHO, Geneva, Switzerland, 2007.

13. Han MK, et al. Chronic obstructive pulmonary disease phenotypes: the future of COPD. Am J Respir Crit Care Med. 2010;182(5):598-604.

14. Sillen MJ, et al. Heterogeneity in clinical characteristics and co-morbidities in dyspneic individuals with COPD GOLD D: findings of the DICES trial. Respir Med. 2013;107(8):1186-94.

15. Vanfleteren LE, et al. Clusters of comorbidities based on validated objective measurements and systemic inflammation in patients with chronic obstructive pulmonary disease. Am J Respir Crit Care Med. 2013;187(7):728-35.

16. Jones R, et al. GOLD COPD categories are not fit for purpose in primary care. Lancet Respir Med. 2013; 1(1): e17.

17. Lange $P$, et al. Prediction of the clinical course of chronic obstructive pulmonary disease, using the new GOLD classification: a study of the general population. Am J Respir Crit Care Med. 2012;186(10):975-81.

18. Agusti A, et al. Characteristics, stability and outcomes of the 2011 GOLD COPD groups in the ECLIPSE cohort. Eur Respir J. 2013;42(3):636-46.

19. Agusti A, et al. FAQs about the GOLD 2011 assessment proposal of COPD: a comparative analysis of four different cohorts. Eur Respir J. 2013;42(5):1391-401.

20. Han MK, et al. GOLD 2011 disease severity classification in COPDGene: a prospective cohort study. Lancet Respir Med 2013;1(1):43-50.

21. Soriano JB, et al. Distribution and prognostic validity of the new Global Initiative for Chronic Obstructive Lung Disease grading classification. Chest. 2013;143(3):694-702.

22. Haughney J, et al. The distribution of COPD in UK general practice using the new GOLD classification. Eur Respir J. 2014;43(4):993-1002.

23. Wilson T, Holt T, Greenhalgh T. Complexity science: complexity and clinical care. BMJ. 2001;323(7314): 685-8.

24. Plsek PE, Greenhalgh T. Complexity science: The challenge of complexity in health care. BMJ. 2001; 323(7313):625-8.

25. Grumbach K. Chronic illness, comorbidities, and the need for medical generalism. Ann Fam Med. 2003; 1(1):4-7.

26. Wagner EH, Austin BT, Von Korff M. Organizing care for patients with chronic illness. Milbank Q. 1996; 74(4):511-44. 
27. Begun JW, Z.B., Dooley KJ. Health care organizations as complex adaptive systems. In: Mick SS, Wyttenback ME, eds. Advances in health care organization theory., 2003. 253(San Francisco: JosseyBass,): p. 2003.

28. Sackett DL, et al. Evidence based medicine: what it is and what it isn't. BMJ. 1996;312(7023):71-2.

29. Timmermans S, Mauck A. The promises and pitfalls of evidence-based medicine. Health Aff (Millwood). 2005;24(1):18-28.

30. Greenhalgh T. Narrative based medicine: narrative based medicine in an evidence based world. BMJ. 1999;318(7179):323-5.

31. Herland K, et al. How representative are clinical study patients with asthma or COPD for a larger "real life" population of patients with obstructive lung disease? Respir Med. 2005;99(1):11-9.

32. Kruis AL, et al. Primary care COPD patients compared with large pharmaceutically-sponsored COPD studies: an UNLOCK validation study. PLoS One. 2014;9(3):e90145.

33. ClinEdQ, Complexity and health care: health practitioner workforce services, roles, skills and training, to respond to patients with complex needs. Clinical Education and Training Queensland, Research and Publication Initiative 2011 CRICOS 00233E, 2011.

34. Ellrodt G, et al. Evidence-based disease management. JAMA. 1997;278(20):1687-92.

35. Hearld LR, et al. Review: how do hospital organizational structure and processes affect quality of care?: a critical review of research methods. Med Care Res Rev. 2008;65(3):259-99.

36. Institute of Medicine, Crossing The Qulity Chasm. National Academy Press, Washington D.C., 2001.

37. Nolte E, McKee M. Caring for people with chronic conditions, A health system perspective. World Health Organization 2008 on behalf of the European Observatory on Health Systems and Policies. 2008.

38. Louise Howarth M, Haigh C. The myth of patient centrality in integrated care: the case of back pain services. Int J Integr Care. 2007; 7:e27.

39. Adams SG, et al. Systematic review of the chronic care model in chronic obstructive pulmonary disease prevention and management. Arch Intern Med. 2007;167(6):551-61.

40. Kruis $\mathrm{AL}$, et al. Integrated disease management interventions for patients with chronic obstructive pulmonary disease. Cochrane Database Syst Rev. 2013;10:CD009437.

41. Kruis AL, et al. Effectiveness of integrated disease management for primary care chronic obstructive pulmonary disease patients: results of cluster randomised trial. BMJ. 2014;349:g5392.

42. Leutz WN. Five laws for integrating medical and social services: lessons from the United States and the United Kingdom. Milbank Q. 1999;77(1):77-110, iv-v.

43. Bodenheimer T, Wagner EH, Grumbach K. Improving primary care for patients with chronic illness. JAMA. 2002;288(14):1775-9.

44. Rijksinstituut voor Volksgezondheid en Milieu (RIVM), Maatschappelijke kosten voor astma, COPD en respiratoire allergie. 2012

45. Lusuardi M, Ambrosini N, Donner CF. Definition and rationale for pulmonary rehabilitation. Pulmonary Rehabilitation. ISBN-10: 0340810173; ISBN-13: 9780340810170, 2005. Part 1: p. 3-8.

46. National Institutes of Health $(\mathrm{NIH})$, Workshop Summary, Pulmonary rehabilitation research. Am J Respir Crit Care Med. 1994;149:825-33.

47. Spruit MA, et al. An official American Thoracic Society/European Respiratory Society statement: key concepts and advances in pulmonary rehabilitation. Am J Respir Crit Care Med. 2013;188(8):e13-64.

48. American Assocation for Respiratory Care (AARC), Clinical practice guideline on pulmonary rehabilitation. Respir Care. 2002;47:(617-25).

49. Agusti A, MacNee W, The COPD control panel: towards personalised medicine in COPD. Thorax. 2013; 68(7):687-90.

50. Agusti A. The path to personalised medicine in COPD. Thorax. 2014;69(9):857-64.

51. Spruit MA, et al. Differences in content and organisational aspects of pulmonary rehabilitation programmes. Eur Respir J. 2014;43(5):1326-37.

52. Baumann HJ, et al. Low intensity, long-term outpatient rehabilitation in COPD: a randomised controlled trial. Respir Res. 2012;13:86.

53. Casey $D$, et al. The effectiveness of a structured education pulmonary rehabilitation programme for improving the health status of people with moderate and severe chronic obstructive pulmonary disease in primary care: the PRINCE cluster randomised trial. Thorax. 2013;68(10):922-8.

54. World Health Organization (WHO), Preparing a health care workforce for the 21st century - the challenge of chronic conditions. WHO, Geneva, Switzerland, 2005. 
55. Spruit MA, et al. Integration of pulmonary rehabilitation in COPD. Lancet. 2008;371(9606):12-3.

56. Amelsvoort P van. The design of work and organisation. Vlijmen, ST-GROEP, 2000.

57. Clegg CW. Sociotechnical principles for system design. Appl Ergon. 2000;31(5):463-77.

58. Porter ME, Lee TH. The Strattegy That Will Fix Health Care. Harvard Business Review, 2013. October.

59. Bohmer RM. The four habits of high-value health care organizations. N Engl J Med. 2011;365(22): 2045-7.

60. Cox K. Clinical practice is not applied scientific method. Aust NZ J Surg. 1995;65:553-7.

61. Mullen PD. Compliance becomes concordance. BMJ. 1997;314(7082):691-2.

62. DiClemente CC, Prochaska JO. Self-change and therapy change of smoking behavior: a comparison of processes of change in cessation and maintenance. Addict Behav. 1982;7(2):133-42.

63. Gustafson D, Cats-Baril W, Alemi F. Systems to support health policy analysis: theory models and uses. Chicago: Health Administration Press, 1992. 



\section{Chapter 4}

\section{The respiratory physiome: clustering based on a comprehensive lung function assessment in patients with COPD}

Ingrid ML Augustin, Martijn A Spruit, Sarah Houben-Wilke, Frits ME Franssen, Lowie EGW Vanfleteren, Swetlana Gaffron, Daisy J A Janssen, Emiel FM Wouters

PLOS ONE 2018;13(9):e0201593. 


\section{Abstract}

\section{Background}

While spirometry and particularly airflow limitation is still considered as an important tool in therapeutic decision making, it poorly reflects the heterogeneity of respiratory impairment in chronic obstructive pulmonary disease (COPD). The aims of this study were to identify pathophysiological clusters in COPD based on an integrated set of standard lung function attributes and to investigate whether these clusters can predict patient-related outcomes and differ in clinical characteristics.

\section{Methods}

Clinically stable COPD patients referred for pulmonary rehabilitation underwent an integrated assessment including clinical characteristics, dyspnea score, exercise performance, mood and health status, and lung function measurements (postbronchodilator spirometry, body plethysmography, diffusing capacity, mouth pressures and arterial blood gases). Self-organizing maps were used to generate lung function based clusters.

\section{Results}

Clustering of lung function attributes of 518 patients with mild to very severe COPD identified seven different lung function clusters. Cluster 1 includes patients with better lung function attributes compared to the other clusters. Airflow limitation is attenuated in clusters 1 to 4 but more pronounced in clusters 5 to 7 . Static hyperinflation is more dominant in clusters 5 to 7. A different pattern occurs for carbon monoxide diffusing capacity, mouth pressures and for arterial blood gases. Related to the different lung function profiles, clusters 1 and 4 demonstrate the best functional performance and health status while this is worst for clusters 6 and 7. All clusters show differences in dyspnea score, proportion of men/women, age, number of exacerbations and hospitalizations, proportion of patients using long-term oxygen and number of comorbidities.

\section{Conclusion}

Based on an integrated assessment of lung function variables, seven pathophysiological clusters can be identified in COPD patients. These clusters poorly predict functional performance and health status. 


\section{Introduction}

Chronic obstructive pulmonary disease (COPD) is a common, preventable and treatable disease that is characterized by persistent respiratory symptoms and airflow limitation that is due to airway and/or alveolar abnormalities usually caused by significant exposure to noxious particles or gases. ${ }^{1}$ While it is widely recognized that COPD is a complex, heterogeneous disease with pulmonary and extra-pulmonary manifestations ${ }^{2}$, post-bronchodilator spirometry remains the diagnostic test to diagnose the disease, classify the degree of airflow limitation ${ }^{1}$, monitor disease progression ${ }^{3}$ and response to pharmacotherapies. ${ }^{4}$ Nevertheless, the degree of airflow limitation correlates only moderately to exercise performance, symptom burden, mood and health status in patients with COPD. ${ }^{5-7}$

Pathophysiology of COPD is far more complex than just airflow limitation. Indeed, lung hyperinflation is one of the hallmarks of patients with COPD. ${ }^{8}$ Lungs can be hyperinflated at rest (static hyperinflation) and/or during exercise (dynamic hyperinflation). ${ }^{9}$ Lung hyperinflation can affect respiratory muscle function in patients with COPD. ${ }^{10}$ Impaired diffusing capacity of the lung is another characteristic in a subgroup of patients with $\mathrm{COPD}^{8}$; when there is a loss of pulmonary capillary bed, as in emphysema, the diffusing capacity falls. Therefore, the single-breath transfer factor of the lung for carbon monoxide (TLCO) is considered as the single best lung function measurement to assess severity of emphysema. ${ }^{8}$ Furthermore, impaired TLCO is one of the strongest predictor of exercise capacity, points out oxygen desaturation during exercise, is highly related to hypoxaemia and poses a high risk for poor survival. ${ }^{11-13}$

Respiratory muscle function has received considerable attention in patients with COPD as many studies have consistently shown that maximal static inspiratory pressures as well as oesophageal pressure are reduced. ${ }^{10}$ These inspiratory muscles are faced to an increased elastic and resistive load in COPD, and the mismatch between the demand for respiratory muscle work and the capacity to meet that demand may partly explain common symptoms in COPD patients as dyspnea, hypercapnia and reduced tolerance to physical exercise. ${ }^{10,14}$ Arterial blood gas measurement is recommended in COPD patients to rule out significant hypoxemia or hypercapnia, particularly in patients with more severe disease. ${ }^{1}$

These lung function measurements offer complementary information but cannot be used individually to accurately predict exercise performance, dyspnea, mood and health status in individual patients with COPD. ${ }^{5}$ Taking into account the heterogeneity of the disease and in an attempt to improve the organization of care for patients with COPD, identifying patient profiles or COPD subtypes by means of clustering analysis has received growing attention. ${ }^{15-17}$ Whether and to what extent a combination of the abovementioned lung function attributes correlates better with patient-related outcomes and clinical traits such as comorbidities was part of our hypothesis. Therefore, 
we aimed to cluster patients with COPD based on solely lung function attributes, derived from post-bronchodilator spirometry, TLCO, whole-body plethysmography, mouth pressures and resting arterial blood gases. A priori, we hypothesized that distinct clusters will be identified showing a large heterogeneity in the combination of lung function attributes in patients with COPD. Moreover, it is hypothesized that significant differences in exercise performance, health status and clinical traits as dyspnea and exacerbations will be found between these pathophysiological clusters, with still a substantial degree of heterogeneity within each of these clusters.

\section{Materials and methods}

\section{Study design}

The current analysis used the data from the Chance Study: an observational, prospective, single-center study about COPD, health status and cardiovascular comorbidities. ${ }^{18}$ This study was approved by the Medical Ethical Committee of the Maastricht University Medical Centre+ (METC 11-3-070) and is registered at http://www.trialregister.nl (NTR 3416) (E-mail: secretariaat.metc@mumc.nl).

\section{Study sample}

Patients with clinically stable COPD ${ }^{1}$ who were referred by a chest physician for a comprehensive pulmonary rehabilitation program at Ciro (Horn, the Netherlands) were eligible to participate. All patients gave written informed consent.

\section{Measurements}

During a 3-day assessment, attributes related to COPD (including lung function), exercise performance, dyspnea, mood and health status were assessed.

\section{Lung function}

Post-bronchodilator spirometry was performed to assess forced expiratory volume in 1 second $\left(F E V_{1}\right)$ and forced vital capacity (FVC). Spirometry was measured with Masterlab ${ }^{\circledR}$ (Jaeger, Würzburg, Germany) following ATS/ERS guidelines. ${ }^{19}$ Values are expressed as percentage of predicted according the Global Lung Function Initiative. ${ }^{20}$ Total lung capacity (TLC), residual volume (RV) and intra thoracic gas volume (ITGV) were determined through body-plethysmography (Masterlab ${ }^{\circledR}$ Jaeger, Würzburg, Germany) following the quality control guidelines. ${ }^{21}$ Values are expressed as a percentage of the European Coal and Steel Community predicted values. ${ }^{22}$ TLCO was measured following the standard of the single-breath determination of carbon monoxide ${ }^{23}$ and expressed in the reference values of Cotes and colleagues. ${ }^{24}$ Additionally, TLCO per unit alveolar 
volume (KCO) was calculated. Maximal static inspiratory (MIP) and expiratory mouth pressures (MEP) were assessed according to ATS/ERS guidelines ${ }^{25}$ and expressed in the reference values according to Black and Hyatt. ${ }^{26}$ Resting arterial partial pressure of oxygen $\left(\mathrm{PaO}_{2}\right)$, carbon dioxide $\left(\mathrm{PaCO}_{2}\right)$ and oxygen saturation were measured (GEM4000, Instrumentation Laboratory, Peachtree City, USA). Patients with long-term oxygen therapy (LTOT) continued oxygen supply during the procedure. All lung function measurements were performed by certified and experienced respiratory technicians.

\section{Clinical, functional and health status characteristics}

As described earlier ${ }^{18}$, smoking history, number of exacerbations and hospitalizations for COPD in the previous twelve months, LTOT, self-reported comorbidities using the Charlson Comorbidity Index $(\mathrm{CCl})^{27}$, the degree of dyspnea using the modified Medical Research Council (mMRC) scale ${ }^{28}$ and disease-specific health status using the COPD Assessment Test (CAT) ${ }^{29}$, the Clinical COPD Questionnaire (CCQ) ${ }^{30}$, and the COPD-specific version of the St George's Respiratory Questionnaire (SGRQ-C) ${ }^{31}$ were assessed. Anxiety and depression were measured by the Hospital Anxiety and Depression Scale (HADS). ${ }^{32}$ Fat-free mass (FFM) was assessed using dual-energy X-ray absorptiometry (Lunar Prodigy system, GE Healthcare, Madison, WI, USA) and FFM was divided by squared height to obtain the FFM-index (FFMI). Low FFMI is defined as an FFMI below $16 \mathrm{~kg} / \mathrm{m}^{2}$ for men and $15 \mathrm{~kg} / \mathrm{m}^{2}$ for women. ${ }^{33}$ Exercise performance was assessed by a 6 -minute walk test (6MWT) and by a symptom limited cardiopulmonary exercise test (CPET) using an electrically, braked cycle ergometer (Carefusion, Houten, the Netherlands) including the measurement of maximal oxygen uptake $\left(\right.$ Peak $\mathrm{VO}_{2} \mathrm{ml} / \mathrm{min}$ ) and maximal work rate in Watts (Peak work rate). Furthermore, a submaximal exercise test at $75 \%$ of the peak work rate (CWRT) was performed. Isokinetic quadriceps muscle strength and endurance were measured using a Biodex (Biodex Medical Systems, Inc., New York, USA).

\section{GOLD classification}

Patients with COPD were classified as GOLD I to IV, and GOLD A to D, according the latest GOLD guideline. ${ }^{1}$

\section{Statistics}

All statistical analyses were performed using Viscovery Profiler 7.1 by Viscovery Software $\mathrm{GmbH}$ (www.viscovery.net; Vienna, Austria). Self-organizing maps (SOMs, also referred to as Kohonen maps) were used to create an ordered representation of the selected attributes. The SOM method can be viewed as a non-parametric regression technique that converts multidimensional data spaces into lower dimensional abstractions. A SOM generates a non-linear representation of the data distribution and allows the user to identify homogeneous data groups visually. Patients have been ordered by their overall similarity concerning the lung function variables $\mathrm{FEV}_{1}$, \% predicted; $F E V_{1} / F V C, \%$; FVC, \% 
predicted; PEF, \% predicted; ITGV, \% predicted; RV, \% predicted; TLC, \% predicted; TLCO, $\%$ predicted; KCO, \% predicted; MIP, \% predicted; MEP, \% predicted and arterial blood gases $\left(\mathrm{PaO}_{2}, \mathrm{PaCO}_{2}\right)$ as well as $\mathrm{SaO}_{2}, \%$ and to a small extent the absolute measures of $\mathrm{FEV}_{1}$; FVC; PEF; ITGV; RV; TLC; TLCO; KCO; TLCHe; VIN; TA; MIP; and MEP measured during pre-rehabilitation assessment. Based on the created SOM model, clusters have been generated using the SOM-Ward Cluster algorithm of Viscovery, a hybrid algorithm that applies the classical hierarchical method of Ward on top of the SOM topology. Summary variables on clinical characteristics for the study sample and for each cluster are presented as mean \pm standard deviation for quantitative variables, and percentage for discrete variables. Viscovery automatically identified for each cluster all patient characteristics that differ significantly from the average of the whole study sample of 518 patients using the integrated two-sided t test with a confidence of $95 \%$.

\section{Results}

\section{Characteristics of the whole sample}

Table 4.1 summarizes the characteristics of the whole sample of 518 patients. As a group, patients demonstrated marked airflow limitation and static hyperinflation. For the total group, TLCO was reduced with normal mean arterial blood gas values. Furthermore, patients generally had a normal body composition, MIP and MEP within normal ranges, an impaired exercise performance, deconditioned quadriceps muscles, and a poor health status. $24 \%$ of the patients used LTOT. The mean number of exacerbations as well as hospitalizations in the last year was on average 2.2 and 0.9 . The majority of these patients was classified as GOLD D. Female COPD patients were younger, more hyperinflated and had worse gas exchange parameters than the male patients. Furthermore, higher symptoms of anxiety were seen in women compared to men. 
Table 4.1 Lung function, clinical, functional and health status characteristics of the whole sample.

\begin{tabular}{|c|c|c|c|c|c|}
\hline & $\begin{array}{c}\text { Whole } \\
\text { sample } \\
n=518\end{array}$ & $\begin{array}{l}\text { Female } \\
n=230\end{array}$ & $\begin{array}{l}\text { Male } \\
n=288\end{array}$ & $\begin{array}{c}\text { p-value } \\
\text { parametric }\end{array}$ & $\begin{array}{c}\text { p-value } \\
\text { non- } \\
\text { parametric }\end{array}$ \\
\hline Women, \% & 44 & & & & \\
\hline Age, years & $64.1(9.1)$ & 62.5 (8.9) & $65.4(9.1)$ & $<0.001$ & $<0.001$ \\
\hline $\mathrm{FEV}_{1}, \%$ predicted & $48.6(20)$ & $49.1(19)$ & $48.2(20)$ & 0.628 & 0.448 \\
\hline $\mathrm{FEV}_{1} / \mathrm{FVC}, \%$ & $37.5(12.2)$ & $38.3(11.8)$ & $36.9(12.6)$ & 0.182 & 0.114 \\
\hline ITGV, \% predicted & 148.6 (35.9) & 152.6 (33.9) & $145.6(37.0)$ & 0.033 & 0.029 \\
\hline RV, \% predicted & $161(50.7)$ & $168.4(48.2)$ & 155.4 (51.9) & 0.005 & 0.002 \\
\hline TLC, \% predicted & $117.1(17.5)$ & $122.4(16.5)$ & $113.1(17.2)$ & $<0.001$ & $<0.001$ \\
\hline TLCO, \% predicted & $49(17)$ & $47.8(15.5)$ & $50.5(18.3)$ & 0.082 & 0.094 \\
\hline $\mathrm{KCO}, \%$ predicted & 64 (21.9) & $59.9(19.3)$ & $67.1(23.2)$ & $<0.001$ & $<0.001$ \\
\hline MIP, \% predicted & $78.5(23.3)$ & $87.0(25.0)$ & $71.7(19.3)$ & $<0.001$ & $<0.001$ \\
\hline MEP, \% predicted & $63.2(20.4)$ & $68.1(22.2)$ & $59.0(17.7)$ & $<0.001$ & $<0.001$ \\
\hline $\mathrm{PaCO}_{2}, \mathrm{kPa}$ & $5.3(0.9)$ & $5.4(0.9)$ & $5.2(0.9)$ & 0.021 & 0.014 \\
\hline $\mathrm{PaO}_{2}, \mathrm{kPa}$ & $9.5(1.5)$ & $9.5(1.4)$ & $9.6(1.5)$ & 0.583 & 0.569 \\
\hline $\mathrm{SaO}_{2}, \%$ & $93.9(3.2)$ & $93.8(3.2)$ & $93.9(3.2)$ & 0.530 & 0.237 \\
\hline Exacerbations $<1$ year, $\mathrm{n}$ & $2.2(1.8)$ & $2.4(1.8)$ & $2.1(1.8)$ & 0.054 & 0.041 \\
\hline Hospitalizations $<1$ year, $\mathrm{n}$ & $0.9(1.3)$ & $0.9(1.3)$ & $0.9(1.3)$ & 0.811 & 0.894 \\
\hline mMRC dyspnea grade & $2.4(1.0)$ & $2.4(1.0)$ & $2.4(1.1)$ & 0.782 & 0.890 \\
\hline LTOT use, \% & 24.1 & 25.7 & 22.9 & & 0.470 \\
\hline Pack years & $42.4(23.6)$ & $41.0(22.7)$ & $43.5(24.2)$ & 0.233 & 0.413 \\
\hline $\mathrm{CCl}$, points & $1.6(1.0)$ & $1.5(0.8)$ & $1.8(1.1)$ & 0.001 & 0.002 \\
\hline Patients with GOLD I / II /III / IV , \% & $7 / 36 / 37 / 20$ & $6 / 38 / 39 / 17$ & $8 / 34 / 35 / 22$ & & 0.354 \\
\hline Patients with GOLD A / B / C / D , \% & $3 / 20 / 5 / 72$ & $2 / 17 / 3 / 79$ & $4 / 23 / 7 / 67$ & & 0.010 \\
\hline 6MWD, m & $424(124.4)$ & 412.9 (118.9) & $432.9(128.1)$ & 0.071 & 0.068 \\
\hline 6MWD, \% predicted & $67.1(18)$ & $70.1(17.3)$ & $64.7(18.1)$ & 0.001 & $<0.001$ \\
\hline Peak $\mathrm{VO}_{2}, \%$ predicted & $66.2(30.4)$ & $85.3(32.8)$ & $51.5(17.7)$ & $<0.001$ & $<0.001$ \\
\hline Peak work rate, \% predicted & $55.5(27.4)$ & $70.1(29.7)$ & $44.1(18.7)$ & $<0.001$ & $<0.001$ \\
\hline CWRT, s & 295.5 (218.7) & $264.5(177.4)$ & $319.3(243.4)$ & 0.006 & 0.022 \\
\hline Quadriceps peak torque, \% predicted & $66.2(18.9)$ & $65.8(18.6)$ & $66.6(19.1)$ & 0.667 & 0.438 \\
\hline $\mathrm{BMI}, \mathrm{kg} / \mathrm{m}^{2}$ & $26.2(5.8)$ & $25.9(5.8)$ & $26.5(5.8)$ & 0.196 & 0.147 \\
\hline FFMI, $\mathrm{kg} / \mathrm{m}^{2}$ & $17.2(2.6)$ & $15.6(2.1)$ & $18.4(2.3)$ & $<0.001$ & $<0.001$ \\
\hline HADS-A, points & $7.8(4.5)$ & $8.7(4.8)$ & $7.1(4.1)$ & $<0.001$ & $<0.001$ \\
\hline HADS-D, points & $7.5(4.3)$ & $7.9(4.7)$ & $7.3(4.0)$ & 0.106 & 0.202 \\
\hline SGRQ, total score, points & $61.1(17.4)$ & $62.5(16.8)$ & $60.0(17.8)$ & 0.105 & 0.085 \\
\hline CAT, total score, points & $21.5(6.6)$ & $22.7(6.1)$ & 20.6 (6.9) & $<0.001$ & 0.001 \\
\hline $\mathrm{CCQ}$, total score, points & $2.6(1.0)$ & $2.7(1.0)$ & $2.6(1.0)$ & 0.102 & 0.080 \\
\hline
\end{tabular}

Data are presented as mean (SD). FEV 1 , forced expiratory volume in $1 \mathrm{~s}$; FVC, forced vital capacity; ITGV, intra thoracic gas volume; RV, residual volume; TLC, total lung capacity; KCO, the single-breath transfer factor of the lung for carbon monoxide (TLCO) per unit alveolar volume; MIP, maximal static inspiratory mouth pressure and $\mathrm{MEP}$, maximal static expiratory mouth pressure; $\mathrm{PaO}_{2}$, arterial partial pressure of oxygen and $\mathrm{PaCO}_{2}$, arterial partial pressure of carbon dioxide; $\mathrm{SaO}_{2}$, arterial oxygen saturation; mMRC, modified Medical Research Council; LTOT, long-term oxygen therapy; CCl, Charlson Comorbidity Index; 6MWD, 6-minute walk distance; $\mathrm{VO}_{2}$ : oxygen uptake; CWRT, constant work-rate test; BMI, body mass index; FFMI, fat-free mass index; HADS-A, Hospital Anxiety and Depression scale, anxiety scores; HADS-D, Hospital Anxiety and Depression scale, depression scores; SGRQ, St. George's Respiratory Questionnaire; CAT, COPD Assessment Test; CCQ, Clinical COPD Questionnaire. 


\section{The lung function clusters}

SOMs resulted in seven clusters with significantly different lung function profiles (Figure 4.1). As shown in Table 4.2 and Figure 4.2, a clear dichotomy is depicted for the spirometric (higher in clusters 1 to 4 ; lower in clusters 5 to 7) and static lung volumes (higher in clusters 5 to 7 ; lower in clusters 1, 2, and 4).

Cluster 1 had a significantly lower degree of airflow limitation, absence of static hyperinflation, and a higher diffusing capacity compared to the other clusters. Clusters 2 to 4 had similar degree of airflow limitation, but showed significant differences in static lung volumes (cluster $3>$ cluster $4>$ cluster 2). Cluster 5 had significantly higher spirometric lung volumes compared to clusters 6 and 7. Static lung volumes were significantly different between clusters 5 to 7 (cluster $7>$ cluster $6>$ cluster 5 ). A differential pattern occurred for TLCO (higher in clusters 1, 4 and 5; lower in clusters 3, 6, and 7) and mouth pressures (higher in clusters 1, 3, 4, and 6; lower in clusters 2, 5, and 7 ). Arterial blood gas values were within normal ranges in all clusters except of cluster 7.

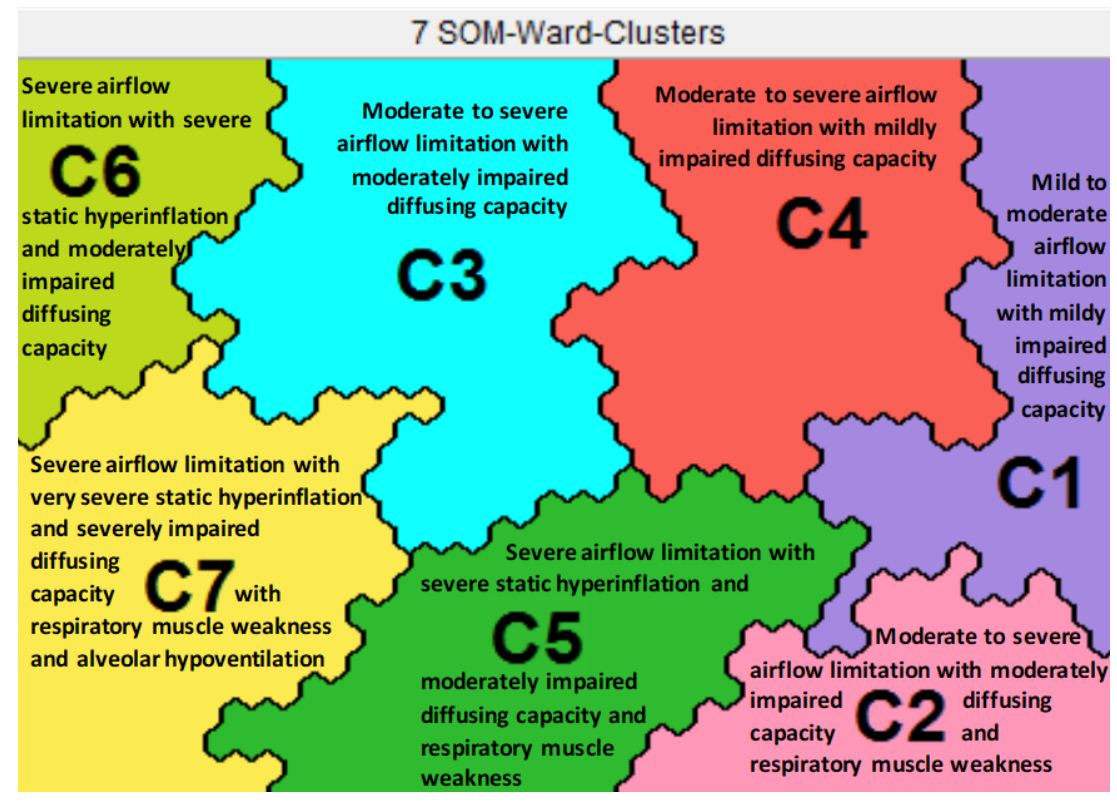

Figure 4.1 Heterogeneity of lung function impairment in COPD. 


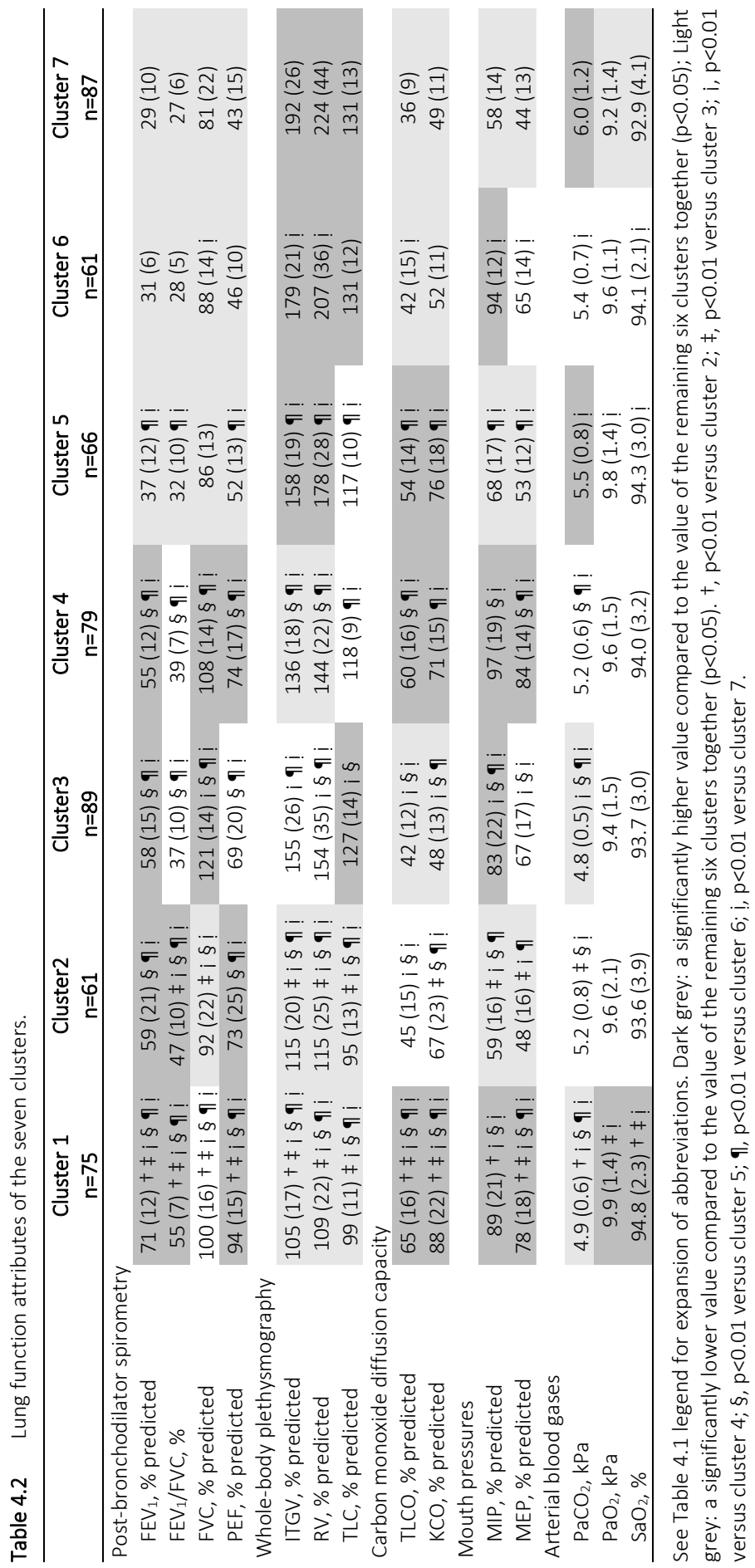



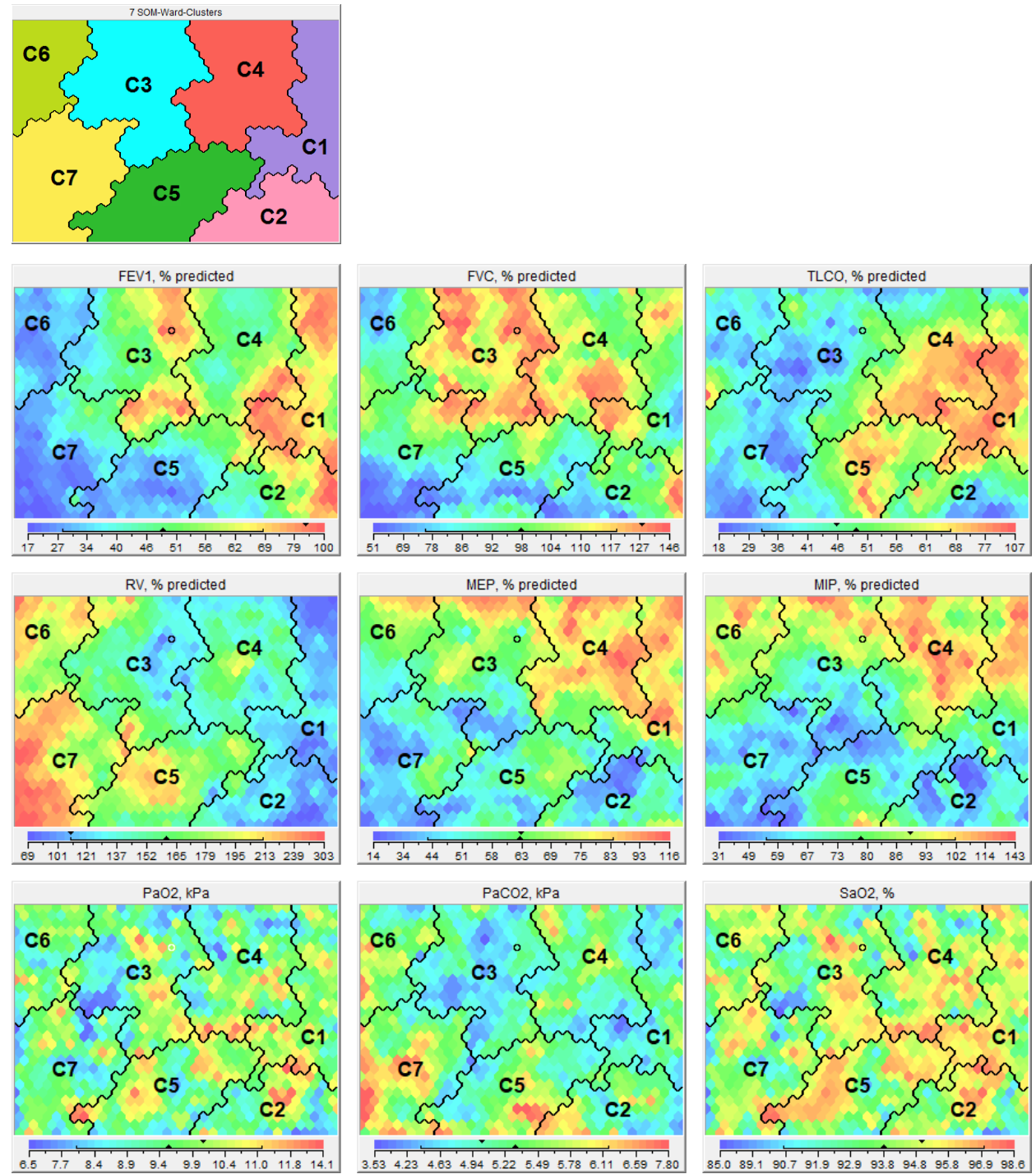

Figure 4.2 The seven lung function clusters in chronic obstructive pulmonary disease (COPD) and the related functional and health status characteristics. When looking at the different lung function, functional characteristics and health status, subjects "raise a red flag" if the attribute is relatively high within this sample, present "a green flag" if the clinical attribute is moderate, and present "a blue flag" when the clinical attribute is relatively low within this sample. In this way the maps can be interpreted. The Viscovery program placed all subjects on a specific position on the map 

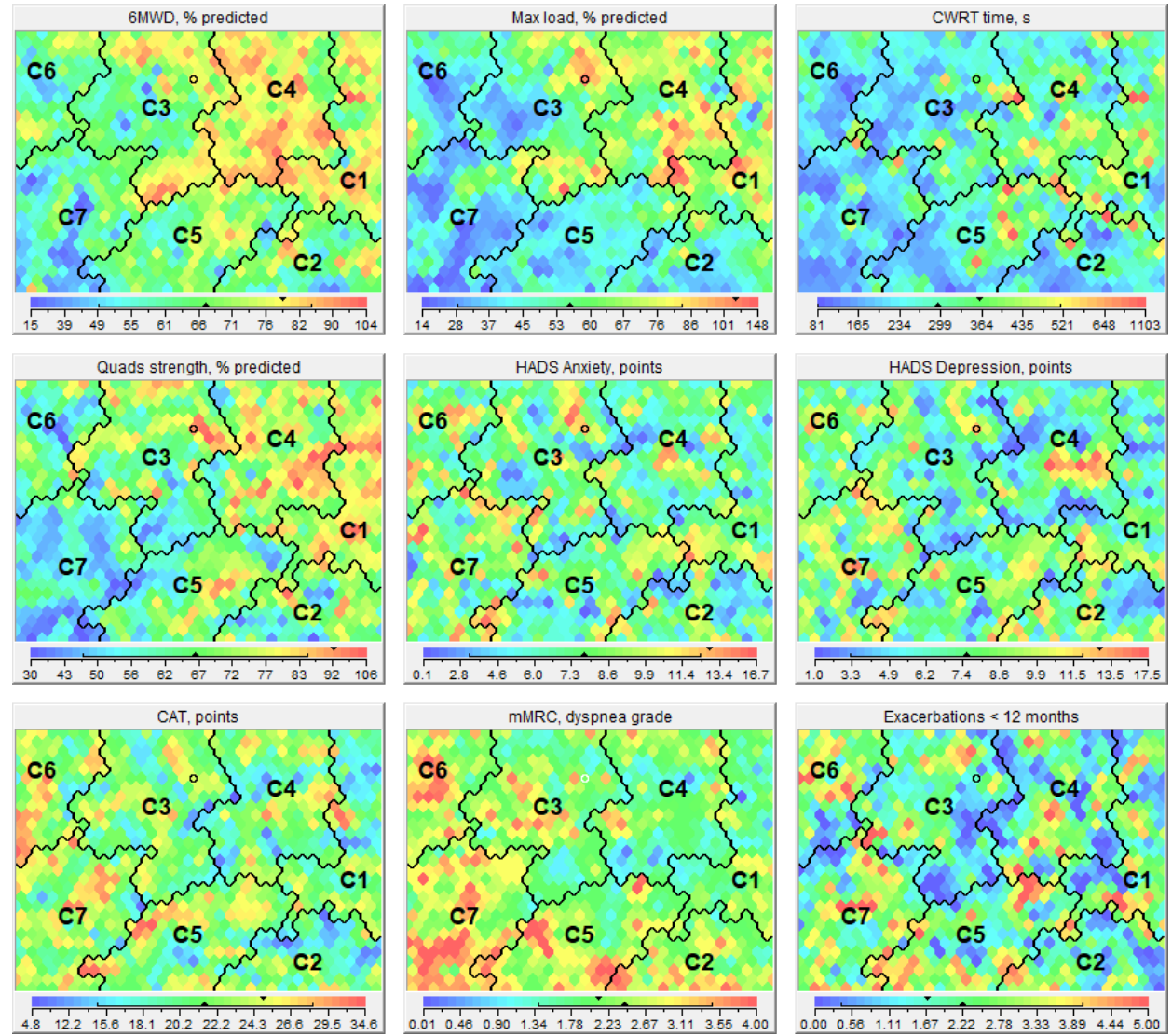

based on their profile of a comprehensive lung function assessment. The more subjects resemble in terms of their lung function the closer they are on the map. Contrarily, the more they differ the further they are away from each other. By drawing lines on the map, the Viscovery program could identify seven different clusters of patients with COPD with a significant different respiratory physiome (95\% confidence interval). 


\section{Functional and health status characteristics of clusters}

Table 4.3 and Figure 4.2 show the functional characteristics and health status related to the seven different lung function profiles. Clusters 1 and 4 generally had the best scores for attributes related to physical fitness (i.e., $6 \mathrm{MWD}$, peak $\mathrm{VO}_{2}$, peak work rate, and quadriceps muscle function) and health status questionnaires (SGRQ, CAT, and CCQ), while this was worst for Clusters 6 and 7.

\section{Clinical characteristics and GOLD classification of clusters}

The clinical characteristics of the seven clusters are summarized in Table 4.4. Clusters 2 and 5 were older and had a higher proportion of men while cluster 6 had a higher proportion of women, as did cluster 3, with a younger mean age. Exacerbations in the last 12 months were higher in clusters 5 and 7, while this was lower in cluster 3. A similar pattern was observed for hospitalizations (higher in cluster 7; lower in clusters 3 and 4). The proportion of patients using long-term oxygen was higher in cluster 7 , and lower in clusters 1 and 4 . Clusters 1 and 2 had higher scores on the Charlson comorbidity index, which was lower in cluster 4. Clusters 6 and 7 had a higher mean dyspnea score. Remarkably, about one quarter of the patients in clusters 1, 3 and 4 were classified as GOLD B and about half of the patients in cluster 1 to 4 were COPD GOLD D patients. Otherwise, practically all patients of clusters 5 to 7 were classified as GOLD D.

Mean scores for anxiety and depression were not significantly different between clusters. As expected from the lung function attributes, clear differences were observed in the GOLD classification per cluster. 


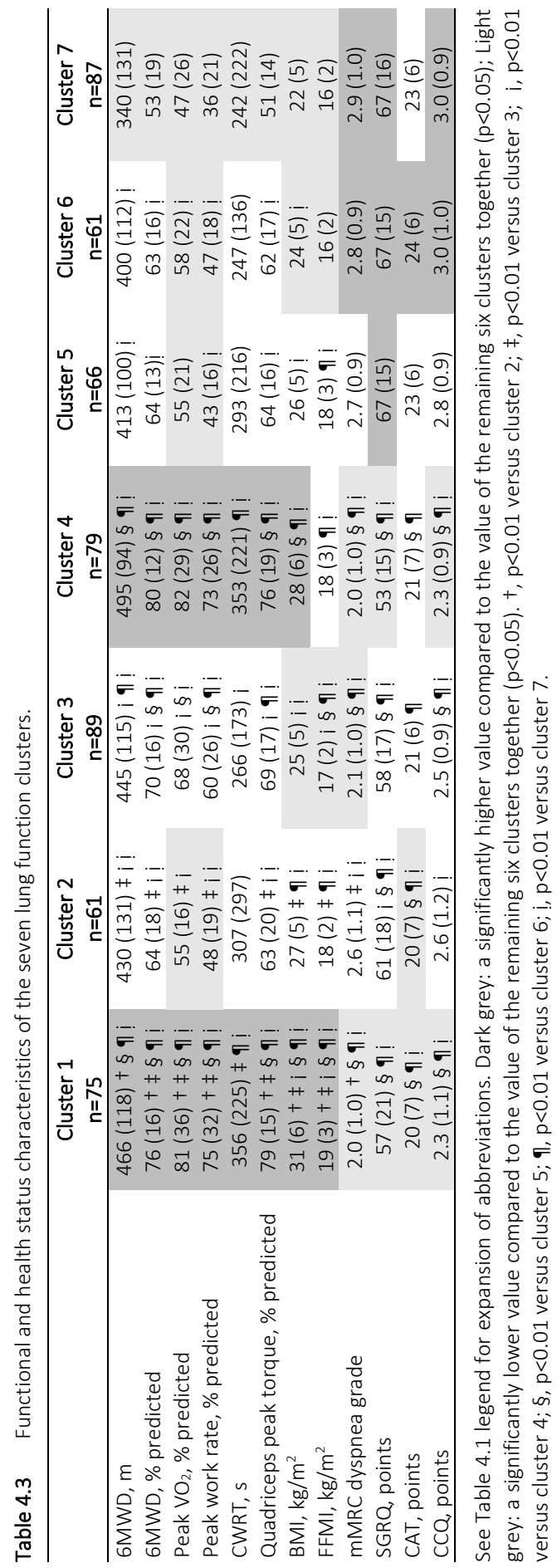




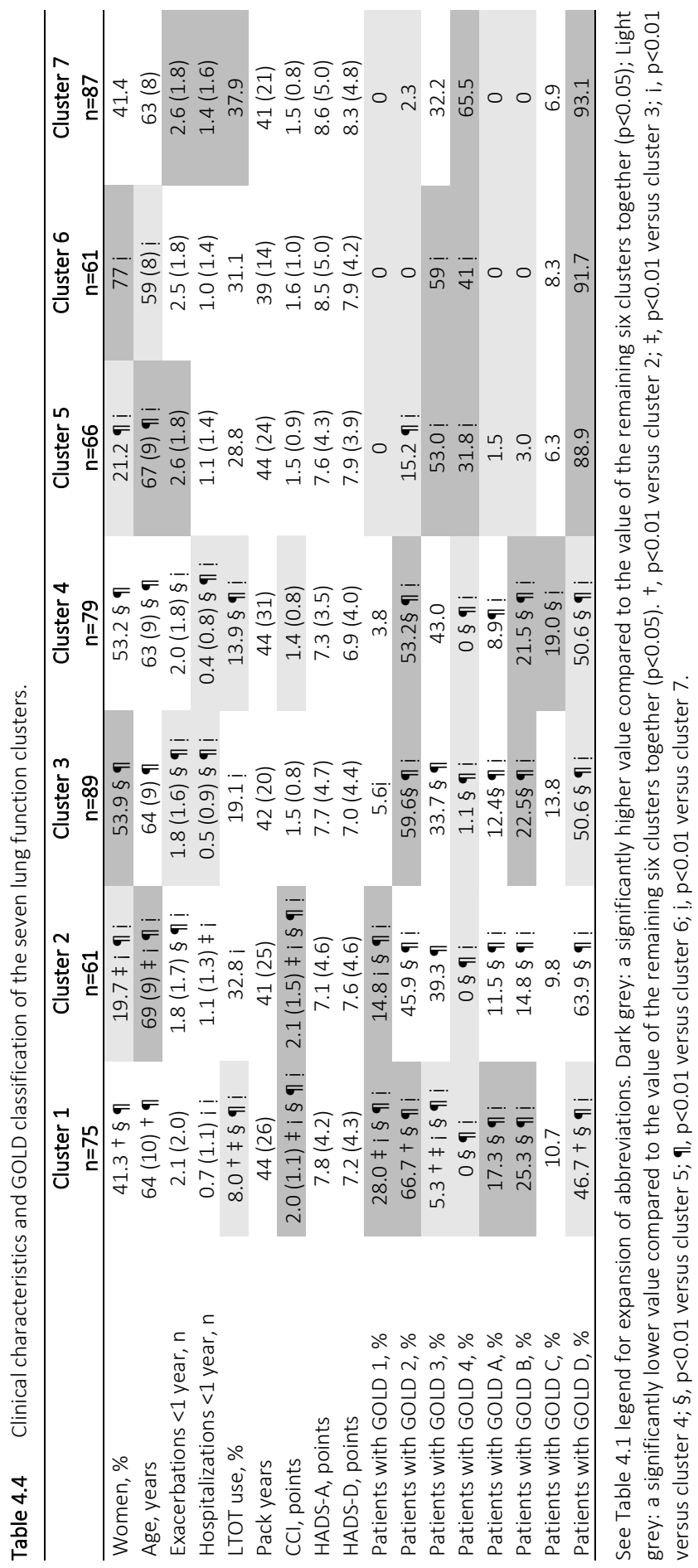




\section{Discussion}

This is the first study clustering patients with mild to very severe COPD based on a comprehensive lung function assessment, including post-bronchodilator spirometry, TLCO, whole-body plethysmography, mouth pressures, and arterial blood gases. Seven clusters were identified, with distinct patterns of lung function impairment demonstrating the complexity and heterogeneity of pathophysiological changes in the respiratory system of COPD patients referred for pulmonary rehabilitation. Our data indicate that simple classification of COPD patients based on spirometry and health status or breathlessness underestimates this heterogeneity in respiratory impairment as well as the identifiable treatable traits in an integrated and individualized management plan for COPD. Significant differences were found in gender distribution, age, exacerbations/hospitalizations, comorbidities, physical fitness, and health status between clusters, only partially related to the degree of lung function impairment. Symptoms of anxiety and depression were comparable between the seven clusters. Large heterogeneity for the abovementioned functional and clinical characteristics still existed within each cluster. Therefore, clustering of lung function attributes does still not allow to accurately determine functional characteristics and health status in individual patients with COPD. These findings emphasize the need of a comprehensive assessment of patients with COPD to gain insight in the different respiratory and systemic treatable traits of the disease in the individual patient in order to understand the true burden of the disease.

Clusters with the best functional performance and health status (clusters 1 and 4) had the lowest extent of airflow limitation, alveolo-capillary membrane damage, the best respiratory muscle function and absent or mild static hyperinflation.

Although GOLD guidelines mention that gas exchange abnormalities result in hypoxemia and hypercapnia, no further recommendation is provided about TLCO measurement to assess the severity, complexity and heterogeneity of COPD. ${ }^{1}$ The current study suggests that quantitative assessment of gas transfer in the lungs offers additional information of respiratory involvement in COPD as part of a standard lung function test. Our study confirms previous findings that reduced TLCO along with airflow limitation identifies those patients with significant more symptoms. ${ }^{11}$ Intriguingly, both clusters with female predominance (clusters 3 and 6) had manifested impaired TLCO.

Lung hyperinflation, the ultimate consequence of expiratory airflow limitation, importantly contributes to the degree of dyspnea, exercise limitation, impaired left ventricular filling and increased cardiovascular mortality associated with the disease. ${ }^{9}$ Our study confirms that clusters with the highest level of static hyperinflation had the worst health and functional status and the highest exacerbation and hospitalization rates, indicating the impact of respiratory mechanics on COPD related disease burden. 
Respiratory muscle function in COPD has received considerable attention in the last decades. Generally, a reduction of MIP is reported in COPD patients. ${ }^{10}$ Intriguingly, our analysis demonstrated a normal respiratory muscle function despite presence of static hyperinflation in cluster 6 while cluster 2 manifested a reduced MIP and MEP despite absence of hyperinflation and absence of nutritional depletion. The same cluster also had manifested lower quadriceps muscle dysfunction and reduced peak exercise performance suggesting underlying intrinsic muscular abnormalities. Stratifying COPD patients based on this heterogeneity of respiratory muscle dysfunction and underlying factors may offer new perspectives for respiratory muscle training as part of an integrated management strategy in these patients.

Interesting are the gender differences between the different clusters with a high prevalence of females in clusters 3 and 6 and a relatively low number of females in clusters 2 and 5 . Clusters 3 and 6 had the most impaired diffusing capacity with normal respiratory muscle strength, opposite to the lung function changes in both male predominant clusters. Furthermore, marked age differences exist between cluster 3 and 6. These data are confirming previous findings of a female predominance in severe, early onset COPD. ${ }^{34}$ Our data also support the findings of Pinto et al, based on a systematic review of clinical phenotypes in COPD. ${ }^{35}$ They describe one phenotype of younger COPD patients with very severe respiratory disease, a low probability of cardiovascular comorbidities, a high prevalence of poor nutritional status and poor health status with poor longitudinal outcomes. ${ }^{35}$ Severely impaired diffusing capacity as illustrated in our analysis seems to be an important pathophysiological characteristic in these patients and offers new therapeutic avenues to treat the disease more aggressively at younger age. Although symptoms for anxiety and depression were comparable between the seven clusters, the presence of higher levels of anxiety and depression in women with COPD may also impact the burden of the disease in these patients. ${ }^{35}$ Also cluster 1 in our study clearly illustrates the limitations of this pathophysiological approach: despite mild impairment of lung function, this cluster of COPD patients had a high disease burden as reflected by worse health status, experienced dyspnea and high rate of even severe exacerbations. This cluster emphasizes the fact that the daily burden of COPD is influenced by factors beyond the lungs and that the presence of comorbidities may explain the impact on health status and functional status. ${ }^{36,37}$

Combined with reported gender differences in clinical presentation, different patterns of comorbidities as well as in response to therapeutic modalities, gender-specific treatment and management strategies must be considered in current medical practice.

Our study clearly illustrates that a variety of pathophysiological respiratory impairments can result in comparable levels of functional impairment, advocating the need for thorough assessment of the individual patient to understand the burden of disease and to select more individualized and targeted intervention strategies. ${ }^{38}$ Recently, a labelfree precision medicine approach for management of chronic airway diseases has been 
proposed based on identification of treatable respiratory, extra-pulmonary and behavior/life style traits. ${ }^{39}$

Considering the outcomes of summative outcome measurements as exercise performance tests as well as health status measurements, our study clearly illustrates that a variety of pathophysiological respiratory impairments can result in comparable levels of functional impairment, advocating the need for thorough assessment of every patient to understand the level of physical functioning and to select more individualized and targeted intervention strategies. ${ }^{39}$ Our data properly emphasizes that selection or restriction of pulmonary rehabilitation cannot be based on one single lung function characteristic as formulated in international recommendations for management of stable COPD. ${ }^{1}$ Such guidelines completely ignore that patients greatly differ in terms of how this complex disease can affect their lives.

\section{Methodological considerations}

The current study has several strengths: 1) a total of 518 well-characterized patients with COPD were analyzed, including patients with GOLD stages 1 to 4 , and A to D; 2 ) the SOMs allowed us to visualize the ratio between the various lung function attributes and attributes related to clinical and functional characteristics and health status, which extends our current insights. However, some limitations need to be considered. First, the current sample contained COPD patients who were referred by chest physicians to a comprehensive pulmonary rehabilitation program. Moreover, the majority of these COPD patients were classified in group D. Therefore, the current findings need to be corroborated in different COPD samples. Second, follow-up studies will also be needed to validate our identified clusters in other cohorts as well as transition of clusters over time. ${ }^{17}$ Indeed, four different clusters of lung function trajectories were recently identified in smokers with and without COPD. ${ }^{40}$ Third, given the cross-sectional nature of the clusters, the relevance in terms of long-term outcomes needs also validation in prospective studies. Fourth, only resting hyperinflation was used in the current approach. Dynamic hyperinflation as part of the pathophysiological attributes used for clustering needs to be evaluated. Then again, it is known that the extent of dynamic hyperinflation inversely varies with the level of resting hyperinflation in patients with COPD $^{41}$ assuming that current findings will not be importantly modified. Fifth, diffusing capacity measurements are used as a surrogate marker of alveolar tissue loss related to emphysema. ${ }^{42}$ Future studies need to consider quantification of the degree and distribution of emphysema using advanced imaging procedures as computed tomography. Finally, pulmonary hemodynamics will complement the COPD related changes in the respiratory system. 


\section{Conclusion}

To conclude, patients with COPD can be clustered based on a comprehensive lung function assessment. The current findings clearly show that the $\mathrm{FEV}_{1}$ is not a pars pro toto for the respiratory impairment in patients with COPD. Moreover, $\mathrm{FEV}_{1}$ or any other single lung function parameter cannot be used to predict the functional characteristics and health status. Our study emphasizes the contributing role of different pulmonary function tests and that different pathophysiological mechanisms lead to a comparable level of functional deterioration. So, a comprehensive assessment, including detection of altered pathophysiological mechanisms, should become essential to understand the personal burden in patients with COPD, to identify treatable traits and to understand the heterogeneity of structure-function relationships in COPD patients. 


\section{References}

1. Vogelmeier CF, et al. Global Strategy for the Diagnosis, Management, and Prevention of Chronic Obstructive Lung Disease 2017 Report. GOLD Executive Summary. Am J Respir Crit Care Med. 2017; 195(5):557-82.

2. Han MK, et al. Chronic obstructive pulmonary disease phenotypes: the future of COPD. Am J Respir Crit Care Med. 2010;182(5):598-604.

3. Decramer $\mathrm{M}$, et al. Effect of tiotropium on outcomes in patients with moderate chronic obstructive pulmonary disease (UPLIFT): a prespecified subgroup analysis of a randomised controlled trial. Lancet. 2009;374(9696):1171-8.

4. Wedzicha JA, et al. Analysis of chronic obstructive pulmonary disease exacerbations with the dual bronchodilator QVA149 compared with glycopyrronium and tiotropium (SPARK): a randomised, doubleblind, parallel-group study. Lancet Respir Med. 2013;1(3):199-209.

5. Spruit MA, et al. Determinants of poor 6-min walking distance in patients with COPD: the ECLIPSE cohort. Respir Med. 2010;104(6):849-57.

6. Agusti A, et al. Characterisation of COPD heterogeneity in the ECLIPSE cohort. Respir Res. 2010;11:122.

7. Janssen DJ, et al. Symptoms of anxiety and depression in COPD patients entering pulmonary rehabilitation. Chron Respir Dis. 2010;7(3):147-57.

8. Corbridge T, Irvin C. Pathophysiology of Chronic Obstructive Pulmonary Disease with Emphasis on Physiologic and Pathologic Correlations. In: Casaburi, R. and Petty, T., Principles and practice of pulmonary rehabilitation. Philadelphia, PA: Saunders, 1993:18-32.

9. O'Donnell DE, Webb KA, Neder JA. Lung hyperinflation in COPD: applying physilology to clinical practice. COPD Research \& Practice, 2015. 1:4:1-12.

10. Marchand E, Decramer M. Respiratory muscle function and drive in chronic obstructive pulmonary disease. Clin Chest Med. 2000;21(4):679-92.

11. Farkhooy $A$, et al. Impaired carbon monoxide diffusing capacity is the strongest predictor of exercise intolerance in COPD. COPD. 2013;10(2):180-5.

12. Saure EW, et al. Diffusion capacity and CT measures of emphysema and airway wall thickness - relation to arterial oxygen tension in COPD patients. Eur Clin Respir J. 2016;3:29141.

13. Boutou AK, et al. Lung function indices for predicting mortality in COPD. Eur Respir J. 2013;42(3): 616-25.

14. Crisafulli E, et al. Respiratory muscles training in COPD patients. Int J Chron Obstruct Pulmon Dis. 2007; 2(1):19-25.

15. Garcia-Aymerich J, et al. Identification and prospective validation of clinically relevant chronic obstructive pulmonary disease (COPD) subtypes. Thorax. 2011;66(5):430-7.

16. Burgel PR, Paillasseur JL, Roche N. Identification of clinical phenotypes using cluster analyses in COPD patients with multiple comorbidities. Biomed Res Int. 2014;2014:420134.

17. Esteban C, et al. Chronic Obstructive Pulmonary Disease Subtypes. Transitions over Time. PLoS One. 2016;11(9):e0161710.

18. Smid DE, et al. Impact of cardiovascular comorbidities on COPD Assessment Test (CAT) and its responsiveness to pulmonary rehabilitation in patients with moderate to very severe COPD: protocol of the Chance study. BMJ Open. 2015;5(7):e007536

19. Miller MR, et al. Standardisation of spirometry. Eur Respir J. 2005;26(2):319-38.

20. Quanjer PH, et al. Multi-ethnic reference values for spirometry for the 3-95-yr age range: the global lung function 2012 equations. Eur Respir J. 2012;40(6):1324-43.

21. Coates AL, et al. Measurement of lung volumes by plethysmography. Eur Respir J. 1997;10(6):1415-27.

22. Quanjer PH, et al. Lung volumes and forced ventilatory flows. Eur Respir J. 1993;6 Suppl 16:5-40.

23. Macintyre N, et al. Standardisation of the single-breath determination of carbon monoxide uptake in the lung. Eur Respir J. 2005;26(4):720-35.

24. Cotes JE, et al. Standardization of the measurement of transfer factor (diffusing capacity). Report Working Party Standardization of Lung Function Tests, European Community for Steel and Coal. Official Statement of the European Respiratory Society. Eur Respir J Suppl. 1993;16:41-52.

25. American Thoracic Society/European Respiratory, S., ATS/ERS Statement on respiratory muscle testing. Am J Respir Crit Care Med. 2002;166(4):518-624. 
26. Black LF, Hyatt RE. Maximal respiratory pressures: normal values and relationship to age and sex. Am Rev Respir Dis. 1969;99(5):696-702.

27. Charlson ME, et al. A new method of classifying prognostic comorbidity in longitudinal studies: development and validation. J Chronic Dis. 1987;40(5):373-83.

28. Mahler DA, Wells CK. Evaluation of clinical methods for rating dyspnea. Chest. 1988;93(3):580-6.

29. Jones PW, et al. Development and first validation of the COPD Assessment Test. Eur Respir J. 2009; 34(3):648-54.

30. van der Molen $\mathrm{T}$, et al. Development, validity and responsiveness of the Clinical COPD Questionnaire. Health Qual Life Outcomes. 2003;1:13.

31. Jones PW, Quirk FH, Baveystock CM. The St George's Respiratory Questionnaire. Respir Med. 1991;85 Suppl B:25-31; discussion 33-7.

32. Zigmond AS, Snaith RP. The hospital anxiety and depression scale. Acta Psychiatr Scand. 1983;67(6): 36170.

33. Schols AM, et al. Prevalence and characteristics of nutritional depletion in patients with stable COPD eligible for pulmonary rehabilitation. Am Rev Respir Dis. 1993;147(5):1151-6.

34. Foreman MG, et al. Early-onset chronic obstructive pulmonary disease is associated with female sex, maternal factors, and African American race in the COPDGene Study. Am J Respir Crit Care Med. 2011; 184(4):414-20.

35. Pinto LM, et al. Derivation and validation of clinical phenotypes for COPD: a systematic review. Respir Res. 2015;16:50.

36. Vanfleteren LE, et al. Clusters of comorbidities based on validated objective measurements and systemic inflammation in patients with chronic obstructive pulmonary disease. Am J Respir Crit Care Med. 2013;187(7):728-35.

37. Ziegelstein RC. Personomics. JAMA Intern Med. 2015;175(6):888-9.

38. Spruit MA, et al. An official American Thoracic Society/European Respiratory Society statement: key concepts and advances in pulmonary rehabilitation. Am J Respir Crit Care Med. 2013;188(8):e13-64.

39. Agusti A, et al. Treatable traits: toward precision medicine of chronic airway diseases. Eur Respir J. 2016;47(2):410-9.

40. Ross JC, et al. Longitudinal Modeling of Lung Function Trajectories in Smokers with and without COPD. Am J Respir Crit Care Med. 2018. doi: : p. 10.1164/rccm.201707-14050C [Epub ahead of print].

41. O'Donnell DE, Revill SM, Webb KA. Dynamic hyperinflation and exercise intolerance in chronic obstructive pulmonary disease. Am J Respir Crit Care Med. 2001;164(5):770-7.

42. Shimizu K, et al. Transfer coefficients (Kco) better reflect emphysematous changes than carbon monoxide diffusing capacity (DLCo) in obstructive lung diseases. J Appl Physiol (1985). 2018. 




\section{Chapter 5}

\section{Differential response to pulmonary rehabilitation in COPD: multidimensional profiling}

Martijn A Spruit, Ingrid ML Augustin, Lowie E Vanfleteren, Daisy JA Janssen, Swetlana Gaffron, Herman-Jan Pennings, Frank Smeenk, Willem Pieters, Jan JAM van den Bergh, Arent-Jan Michels, Miriam TJ Groenen, Erica PA Rutten, Emiel FM Wouters, Frits ME Franssen on behalf of the CIRO+ Rehabilitation Network 


\section{Abstract}

The aim of the present study was to profile a multidimensional response to pulmonary rehabilitation in patients with chronic obstructive pulmonary disease (COPD).

Dyspnea, exercise performance, health status, mood status and problematic activities of daily life were assessed before and after a 40-session pulmonary rehabilitation program in 2068 patients with COPD (mean forced expiratory volume in $1 \mathrm{~s}$ of $49 \%$ predicted). Patients were ordered by their overall similarity concerning their multidimensional response profile, which comprises the overall response on MRC dyspnea grade, 6MWD, cycle endurance time, Canadian Occupational Performance Measure performance and satisfaction scores, Hospital Anxiety and Depression Scale anxiety and depression, and St George's Respiratory Questionnaire total score, using a novel non-parametric regression technique.

Patients were clustered into four groups with distinct multidimensional response profiles: $n=378$ (18.3\%; "very good responder"), $n=742$ (35.9\%; "good responder"), n=731 (35.4\%; "moderate responder"), and n=217 (10.5\%; "poor responder"). Patients in the "very good responder" cluster had higher symptoms of dyspnea, number of hospitalizations <12 months, worse exercise performance, worse performance and satisfaction scores for problematic activities of daily life, more symptoms of anxiety and depression, worse health status, and a higher proportion of patients following an inpatient PR program compared to the other three clusters.

A multidimensional response outcome needs to be considered to study the efficacy of pulmonary rehabilitation services in patients with COPD, as responses to regular outcomes are differential within patients with COPD. 


\section{Introduction}

Pulmonary rehabilitation (PR) is a comprehensive intervention designed to improve the physical and psychological condition of people with chronic respiratory disease. ${ }^{1}$ Daily symptoms, exercise performance and health status generally improve following PR. ${ }^{2}$ Therefore, PR is recognized as a fundamental part of the integrated care of people with chronic obstructive pulmonary disease (COPD). ${ }^{3}$

Since financial resources for PR are often limited or even non-existing ${ }^{4}$, identification of (clusters of) patients that do (or do not) respond to PR will become necessary in the near future to improve its cost-effectiveness. This requires consensus about the key performance measures of PR services. To date, changes in exercise performance and health status are often used to qualify individuals with COPD as responders or nonresponders to PR. ${ }^{5-10}$ Nevertheless, changes in the abovementioned outcomes following $P R$ are mostly differential. Indeed, patients may improve health status without an improvement in exercise capacity or vice versa; and patients may improve walk distance without an improvement in cycle endurance or vice versa..$^{811-13}$ So, the choice for exercise performance and/or health status as key performance measures seems too simple, and the use of non-linear statistics seems inevitable. Moreover, multiple other outcomes (i.e., symptoms of dyspnea, cycle endurance time, performance of problematic activities of daily life, and symptoms of anxiety and depression ${ }^{2,12,14,15}$ have been identified by health care professionals as essential to evaluate the efficacy of PR services. ${ }^{4}$ So, a comprehensive evaluation of the efficacy of PR in individuals with COPD is complex, and requires a thorough initial and outcome assessment. ${ }^{1,16}$ Therefore, we sought to profile a multidimensional response to PR in patients with COPD, including symptoms of dyspnea, exercise performance, health status, mood status, and problematic activities of daily life, using a non-parametric regression technique.

\section{Methods}

\section{Patients}

We extracted data from the Integrated Knowledge System based on BioXM ${ }^{\mathrm{TM}}$ (Biomax Informatics AG, Munich, Germany) of 3349 patients with the diagnosis of COPD who were evaluated during the initial assessment of a comprehensive PR program at Ciro, center of expertise for chronic organ failure in Horn (The Netherlands) between January 2006 to December 2012. ${ }^{16}$ Of these records, 706 patients dropped out during the PR, while 575 patients had 5 or more missing values for the response indicators (please see below for more details) at baseline and/or outcome assessment. Finally, 2068 patients (42.9\% women) met the following inclusion criteria: a primary diagnosis of COPD, a postbronchodilator forced expiratory volume in $1 \mathrm{~s} /$ forced vital capacity ratio of $\leq 0.70$, and completion of PR. This analysis also included patients with exacerbations prior to and/or 
during the study. Ethical approval was not indicated because all of the tests were done as part of the routine initial assessment ${ }^{16}$, and analyzed retrospectively. The Board of Directors of Ciro approved the use of de-identified patients' records.

\section{Testing}

As part of routine 3 -day initial assessment ${ }^{16}$, patients underwent, amongst other tests and questionnaires, a maximal incremental cycle test during which peak work rate was determined. Subsequently, on a different day, patients performed a constant work rate test at $75 \%$ of the determined peak work rate. ${ }^{17}$ Patients also performed two 6 -min walk tests $^{18}$ and the test with the longest 6-min walk distance (6MWD) was used for further analysis. ${ }^{19,20}$ Spirometry, physical examination, medical history and Medical Research Council (MRC) dyspnea scale data were obtained. Patients underwent an intake by an occupational therapist, including the Canadian Occupational Performance Measure (COPM) to identify and discuss specific problematic activities of daily life. ${ }^{21}$ In addition, patients identified their perception of how well they were performing the problematic activities of daily life (performance score; COPM-P) and how satisfied they were with this level of performance (satisfaction score; COPM-S). These scores were ascertained by using the cue cards to identify a score between 1 ("not able to do it" or "not at all satisfied", respectively) to 10 points ("able to do it extremely well" or "extremely satisfied"). The COPM is reliable in COPD ${ }^{22}$ and responsive to PR. ${ }^{15}$ Mood status has been assessed using the Hospital Anxiety and Depression Scale (HADS). ${ }^{23}$ HADS is divided in an anxiety subscale (HADS-A) and a depression subscale (HADS-D). Total scores for each subscale can range from 0 (optimal) to 21 (worst) points. A score from 8 to 10 indicates a mild mood disturbance, a score from 11 to 14 a moderate mood disturbance and a score from 12 to 21 a severe mood disturbance. ${ }^{23}$ For assessment of disease-specific health status, the St. George Respiratory Questionnaire (SGRQ) has been used. The SGRQ consists of 50 items, divided in three domains (symptoms, activities and impact), providing three domain scores. A total score is also provided (SGRQ-T). Scores can range from 0 (optimal) to 100 points (worst). ${ }^{24}$

\section{Intervention}

All patients underwent PR, as described previously. ${ }^{25}$ In brief, Ciro provides a state-of-theart interdisciplinary PR program for patients with COPD consisting of 40 sessions, in line with the 2013 American Thoracic Society/European Respiratory Society Statement on PR. ${ }^{1}$ PR can be inpatient ( 8 weeks, 5 days/week) or outpatient ( 8 weeks, 3 half days/week, followed by 8 weeks 2 half days/week). The outpatient PR programs took place in the Ciro rehabilitation network. During baseline assessment, a careful characterization of the extra-pulmonary features of patients with COPD was performed, which determined the application of various treatments: physical exercise training, occupational therapy, nutritional counselling, psychosocial counselling, education and exacerbation management. Physical exercise training was the cornerstone of the program, consisting of 
strengthening exercises, treadmill walking and stationary cycling. All exercises were performed at moderate-to-high intensity to obtain an overload stimulus. Moreover, the training intensity increased during the rehabilitation period, based on dyspnea and fatigue symptom scores. All patients underwent flexibility exercises, general physical exercise for lower and upper extremities, and daily supervised 30-min outdoor walks. Patients, who were too dyspneic to perform endurance/interval/resistance training, received lower-limb high-frequency neuromuscular electrical stimulation. ${ }^{26}$

\section{Statistics}

Data were expressed as mean (standard deviation) or as frequency, as appropriate. Moreover, the patient data were ordered based on the overall similarity concerning selected attributes. The attributes that drove the ordering process of the patients in the map are the overall response and the differences in the response indicators. The overall response is based on the weighted standardized differences between initial and outcome assessment of all eight response indicators: MRC, 6MWD, cycle endurance time, COPM-P, COPM-S, HADS-A, HADS-D, and SGRQ-T. Thus, patients with a similar response profile are placed closed to each other in the map. Based on the ordering of the patients in the map, the hierarchical ward cluster algorithm has been applied, to cluster the patients into 4 response clusters. The values of all attributes included in the analyses could then be recalled cluster by cluster to be exported for the statistical tables. For the clustering of patients the Viscovery ${ }^{\circledR}$ Data Mining Suite, version 6.1 by Viscovery Software GmbH (www.viscovery.net) was used, which is based on the technology of selforganizing maps (SOMs, also referred to as Kohonen maps). SOMs represent an ordered representation of multidimensional data which simplifies complexity and reveals meaningful relationships, and have been used before in COPD. ${ }^{27}$

Four clusters of patients with substantially different response profiles have been generated. The efficacy of the pulmonary rehabilitation program has been evaluated based on the Minimal Clinically Important Difference (MCID). The following MCIDs were used: -1 grade on MRC dyspnea scale $;+30$ m on $6 \mathrm{MWD}^{19,20} ;+100$ s on cycle endurance time $^{13} ;+2$ points on COPM-P ${ }^{15} ;+2$ points on COPM-S ${ }^{15} ;-1.5$ points on HADS-A ${ }^{28} ;-1.5$ points on HADS-D ${ }^{28}$; and -4 points on SGRQ-T. ${ }^{29}$

\section{Results}

\section{Baseline characteristics}

On average, patients had moderate-to-very severe COPD, an impaired exercise capacity, a poor health status, and experienced problems during the performance of activities of daily life. Moreover, patients were on multiple pulmonary and non-pulmonary drug treatments (Supplemental Table S5.1). Patients with long-term oxygen therapy generally had more 
symptoms of dyspnea, and worse exercise performance, mood status and health status compared with patients without long-term oxygen therapy (Table 5.1).

Table 5.1 Baseline characteristics.

\begin{tabular}{|c|c|c|c|c|c|}
\hline Baseline & All patients & $\begin{array}{c}\text { Men } \\
\text { without LTOT }\end{array}$ & $\begin{array}{c}\text { Women } \\
\text { without LTOT }\end{array}$ & $\begin{array}{c}\text { Men } \\
\text { with LTOT }\end{array}$ & $\begin{array}{l}\text { Women } \\
\text { with LTOT }\end{array}$ \\
\hline Patients n (\%) & $2068(100)$ & $1012(48.9)$ & $740(35.8)$ & $168(8.1)$ & $148(7.2)$ \\
\hline Age, years & $64(9)$ & $66(9)$ & $61(9)^{*}$ & $66(8)+$ & $65(7)+$ \\
\hline $\mathrm{FEV}_{1}, \mathrm{I}$ & $1.3(0.6)$ & $1.5(0.6)$ & $1.2(0.5)^{*}$ & $1.0(0.4) *+$ & $0.7(0.3) *+9$ \\
\hline $\mathrm{FEV}_{1}, \%$ predicted & 49 (19) & $50(18)$ & $53(18) *$ & $33(9) *+$ & $35(14) *+$ \\
\hline $\mathrm{FEV}_{1} / \mathrm{FVC}, \%$ & $40(12)$ & $41(12)$ & $43(12)^{*}$ & $32(9)^{*+}$ & $34(9)^{*+}$ \\
\hline KCO, \% predicted & $66(23)$ & $71(24)$ & $63(21) *$ & $61(23) *$ & $53(17) *+9$ \\
\hline $\mathrm{PaO}_{2}, \mathrm{kPa}$ & $9.6(1.4)$ & $9.6(1.3)$ & $9.7(1.4)$ & $9.7(1.7)$ & $9.4(1.6)$ \\
\hline $\mathrm{PaCO}_{2}, \mathrm{kPa}$ & $5.3(0.8)$ & $5.2(0.6)$ & $5.2(0.6)$ & $6.0(1.2) *+$ & $6.3(1.1) *+9$ \\
\hline $\mathrm{SaO}_{2}, \%$ & $95.0(2.3)$ & $95.0(2.1)$ & $95.1(2.4)$ & $94.8(2.4)$ & $94.4(2.8)^{*+}$ \\
\hline MRC, grade & $3.3(1.1)$ & $3.1(1.1)$ & $3.2(1.1)$ & $4.2(1.0) *+$ & $4.2(1.1)^{*+}$ \\
\hline Exacerbation. $<12 \mathrm{~m}, \mathrm{n}$ & $2.1(2.4)$ & $1.9(2.4)$ & $2.1(2.2)$ & $3.1(2.7) *+$ & $3.0(2.8) *+$ \\
\hline Admission $<12 \mathrm{~m}, \mathrm{n}$ & $0.7(1.4)$ & $0.5(1.1)$ & $0.6(1.2)$ & $2.1(2.2) *+$ & $1.6(1.5)^{*+}$ \\
\hline CC index, points & $1.4(1.2)$ & $1.5(1.2)$ & $1.2(0.9)^{*}$ & $1.6(1.4)+$ & $1.3(1.0)$ \\
\hline $\mathrm{BMI}, \mathrm{kg} / \mathrm{m}^{2}$ & $25.6(5.3)$ & $25.8(4.95)$ & $25.0(5.5)^{*}$ & $26.2(5.4)$ & $26.0(6.0)$ \\
\hline FFMI, $\mathrm{kg} / \mathrm{m}^{2}$ & $16.7(2.4)$ & $17.7(2.2)$ & $15.3(1.9)^{*}$ & $17.7(2.4)+$ & $15.7(2.4) *$ ๆ \\
\hline $6 \mathrm{MWD}, \mathrm{m}$ & $447(115)$ & $474(111)$ & $452(102) *$ & $357(107) *+$ & $340(105) *+$ \\
\hline 6MWD, \% predicted & $70.3(16.4)$ & $70.8(15.4)$ & $74.5(15.3)^{*}$ & $54.9(15.9) *+$ & $61.5(16.7) *+9$ \\
\hline PWR, watts & $72(31)$ & $83(34)$ & $65(23)^{*}$ & $57(18)^{*+}$ & $46(15) *+9$ \\
\hline PWR, \% predicted & $56.9(25.4)$ & $51.8(21.3)$ & $68.4(28.0) *$ & $36.9(14.1) *+$ & $55.6(21.8)+9$ \\
\hline $\mathrm{VO}_{2}, \%$ predicted & $68.6(31.1)$ & $55.0(15.8)$ & $88.0(36.5)^{*}$ & N.A. & N.A. \\
\hline Ventilation, \%MVV & $84.3(21.4)$ & $84.3(20.8)$ & $84.4(21.5)$ & N.A. & N.A. \\
\hline CWRT, s & $315(234)$ & $354(256)$ & $298(221) *$ & $239(165) *+$ & $211(109) *+$ \\
\hline COPM-P, points & $4.3(1.3)$ & $4.5(1.3)$ & $4.2(1.3) *$ & $3.7(1.4) *+$ & $3.7(1.4) *+$ \\
\hline COPM-S, points & $3.7(1.7)$ & $4.0(1.7)$ & $3.5(1.7)^{*}$ & $3.4(1.6) *$ & $3.2(1.5)^{*}$ \\
\hline HADS-A, points & $7.2(4.3)$ & $6.3(4.0)$ & $8.0(4.3) *$ & $7.7(4.8)^{*}$ & $8.9(4.7) *$ \\
\hline$\geq 8$ points, $\%$ & 44.0 & 34.0 & $52.0 *$ & $54.0^{*}$ & $63.0 * 9$ \\
\hline HADS-D, points & $6.8(4.1)$ & $6.3(3.8)$ & $6.9(4.2)^{*}$ & $7.9(4.3) *+$ & $7.9(4.5)^{*}$ \\
\hline$\geq 8$ points, $\%$ & 41.0 & 36.0 & $42.0^{*}$ & $54.0 *+$ & $54.0 *$ \\
\hline SGRQ-T, points & $53.5(17.0)$ & $51.8(16.9)$ & $52.0(16.9)$ & $63.7(15.0) *+$ & $62.4(13.8)^{*+}$ \\
\hline BODE index, points & $3.5(1.2)$ & $3.1(1.9)$ & $3.1(2.0)$ & $5.5(1.9) *+$ & $5.5(2.1) *+$ \\
\hline ADO index, points & $4.4(1.7)$ & $4.3(1.6)$ & $3.9(1.7) *$ & $6.0(1.3) *+$ & $5.7(1.4) *+$ \\
\hline
\end{tabular}

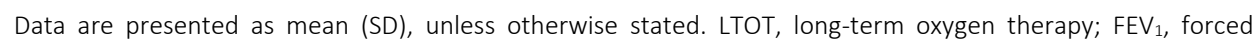
expiratory volume in the first second; FVC, forced vital capacity; KCO, transfer factor of the lung for carbon monoxide; $\mathrm{PaO}_{2}$, arterial oxygen tension; $\mathrm{PaCO}_{2}$, arterial carbon dioxide tension; $\mathrm{SaO}_{2}$, arterial oxygen saturation; MRC, Medical Research Council dyspnea grade; CC index, Charlson Comorbidity Index; BMI, body mass index; FFMI, fat-free Mass Index; 6MWD, 6-min walk distance; PWR, peak work rate; $\mathrm{VO}_{2}$, oxygen uptake; MVV, maximal voluntary ventilation; CWRT, constant work-rate test; COPM-P, Canadian Occupational Performance Measure, performance score; COPM-S, Canadian Occupational Performance Measure, satisfaction score; HADS-A, Hospital Anxiety and Depression Scale, anxiety scores; HADS-D, Hospital Anxiety and Depression Scale, depression scores; SGRQ-T, St. George's Respiratory Questionnaire, total score; BODE, body mass index, airflow obstruction, dyspnoea, exercise capacity; ADO, age, dyspnoea, airflow obstruction, N.A., Not assessed. Missing data in men without long-term oxygen therapy were $M R C, n=33 ; 6 M W D, n=43$; $V_{2}, \mathrm{n}=47$; ventilation, $\mathrm{n}=47$; CWRT, $\mathrm{n}=110$; COPM-P, $\mathrm{n}=106$; COPM-S, $\mathrm{n}=106$; HADS-A, $\mathrm{n}=34$; HADS-D, $n=34$; SGRQ-T, $n=71$. Missing data in women without long-term oxygen therapy: MRC, $n=15 ; 6 M W D, n=33 ; V O_{2}$, $n=49$; ventilation, $n=49$; CWRT, $n=75$; COPM-P, $n=58$; COPM-S, $n=58$; HADS-A, $n=31$; HADS-D, $n=31$; SGRQ-T, $n=53$. Missing data in men with long-term oxygen therapy: MRC, $n=2 ; 6 M W D, n=7 ; C W R T, n=19 ; C O P M-P$, $\mathrm{n}=12$; COPM-S, $\mathrm{n}=12$; HADS-A, $\mathrm{n}=11$; HADS-D, $\mathrm{n}=11 ; \mathrm{SGRQ}-\mathrm{T}, \mathrm{n}=21$. Missing data in women with long-term oxygen therapy: MRC, $n=1 ; 6 M W D, n=7$; CWRT, $n=34$; COPM-P, $n=8$; COPM-S, $n=8$; HADS-A, $n=12$; HADS-D, $\mathrm{n}=12 ; \mathrm{SGRQ}-\mathrm{T}, \mathrm{n}=24 .{ }^{*}, \mathrm{p}<0.01$ versus men without long-term oxygen therapy; ${ }^{+}, \mathrm{p}<0.01$ versus women without long-term oxygen therapy; $\mathfrak{n}, \mathrm{p}<0.01$ versus men with long-term oxygen therapy. 


\section{Response to pulmonary rehabilitation: whole group}

Significant improvements were found for symptoms of dyspnea (MRC: $-0.4 \pm 1.1$ ), 6MWD $(27 \pm 57 \mathrm{~m})$, cycle endurance time $(208 \pm 328 \mathrm{~s})$, performance of problematic activities of daily life (COPM-P: $2.0 \pm 1.7$ points), the satisfaction with the performance of the problematic activities of daily life (COPM-S: $2.6 \pm 2.1$ points), symptoms of anxiety (HADSA: $-1.4 \pm 3.5$ points), symptoms of depression (HADS-D: $-1.4 \pm 3.5$ points), and health status (SGRQ total score: $-5.3 \pm 12.6$ points) (all $p<0.01$ ).

\section{Multidimensional response profiling: whole sample}

The 2068 patients with COPD were clustered into four groups with distinct multidimensional response profiles: $n=378(18.3 \%)$ in the cluster "very good responder", $\mathrm{n}=742(35.9 \%)$ in the cluster "good responder", $\mathrm{n}=731$ (35.4\%) in the cluster "moderate responder", and $n=217$ (10.5\%) in the cluster "poor responder" (Table 5.2). The response to PR was best in the very good responder cluster on all outcome measures compared with the other clusters (Table 5.2, Figure 5.1). Indeed, a clinically relevant improvement was achieved in $85 \%$ of the outcomes of the patients in cluster "very good responder", while this was only achieved in $11 \%$ of the outcome of the patients in poor responder cluster (Table 5.2, Figure 5.2).

\section{Cluster characteristics: whole sample}

Table 5.3 summarizes the baseline characteristics of the patients after stratification for the multidimensional response clusters. Age, the proportion of women, the degree of airflow limitation and the transfer factor for carbon monoxide were comparable between clusters. Patients in the very good responder cluster had significantly higher symptoms of dyspnea, number of hospital admissions in the last 12 months, a worse exercise performance, worse performance and satisfaction scores for problematic activities of daily life, more symptoms of anxiety and depression, a worse health status, and a higher proportion of patients following an inpatient PR program compared with the other three clusters. Moreover, patients from the very good responder cluster had a higher proportion of long-term oxygen therapy users, a higher body mass index and a higher fat-free mass index at baseline compared with the patients of the moderate responder and poor responder clusters. 

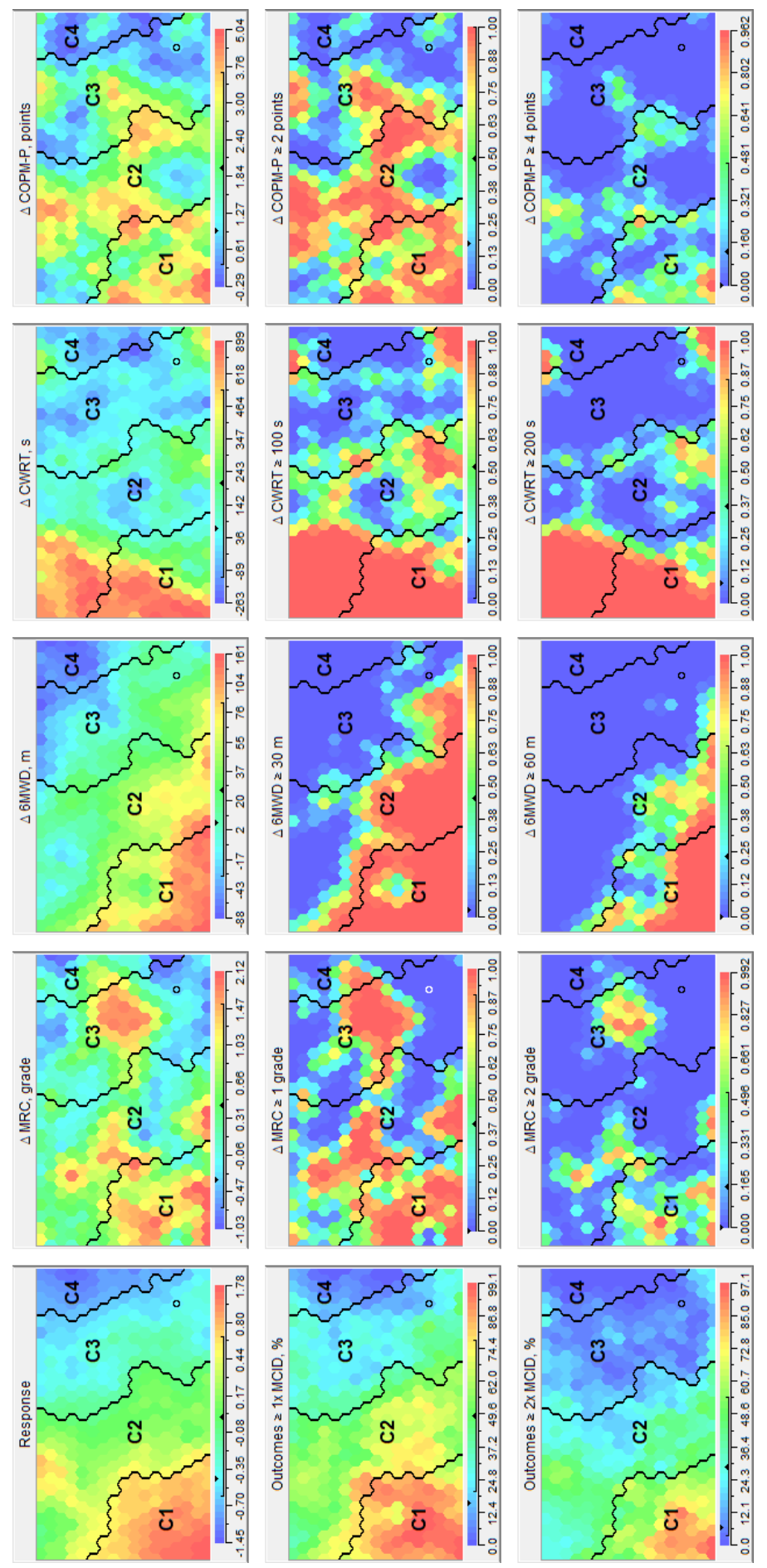

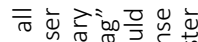
등은 등 언 유은

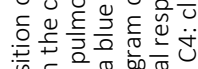
กั 든

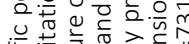

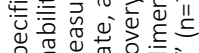
กㄴㄷㄴ ᄃㄴำ ठ

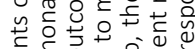

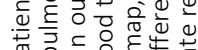
苋至穴

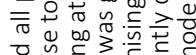

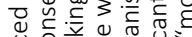

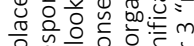

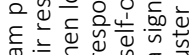
厄. क力م)

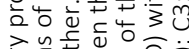

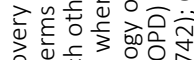
원동엉 出.

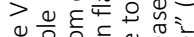

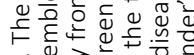
त्र 语施心

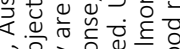

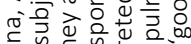

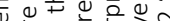
> 히웜웡

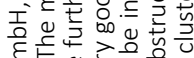
نั 눈 인

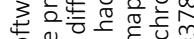
ค

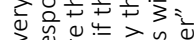

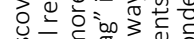

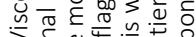
$\sum \frac{ᄃ}{2}$

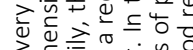

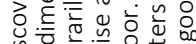

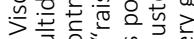
مอ

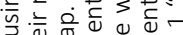

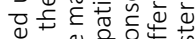

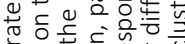

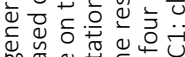

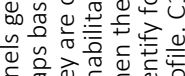

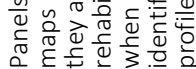

군 

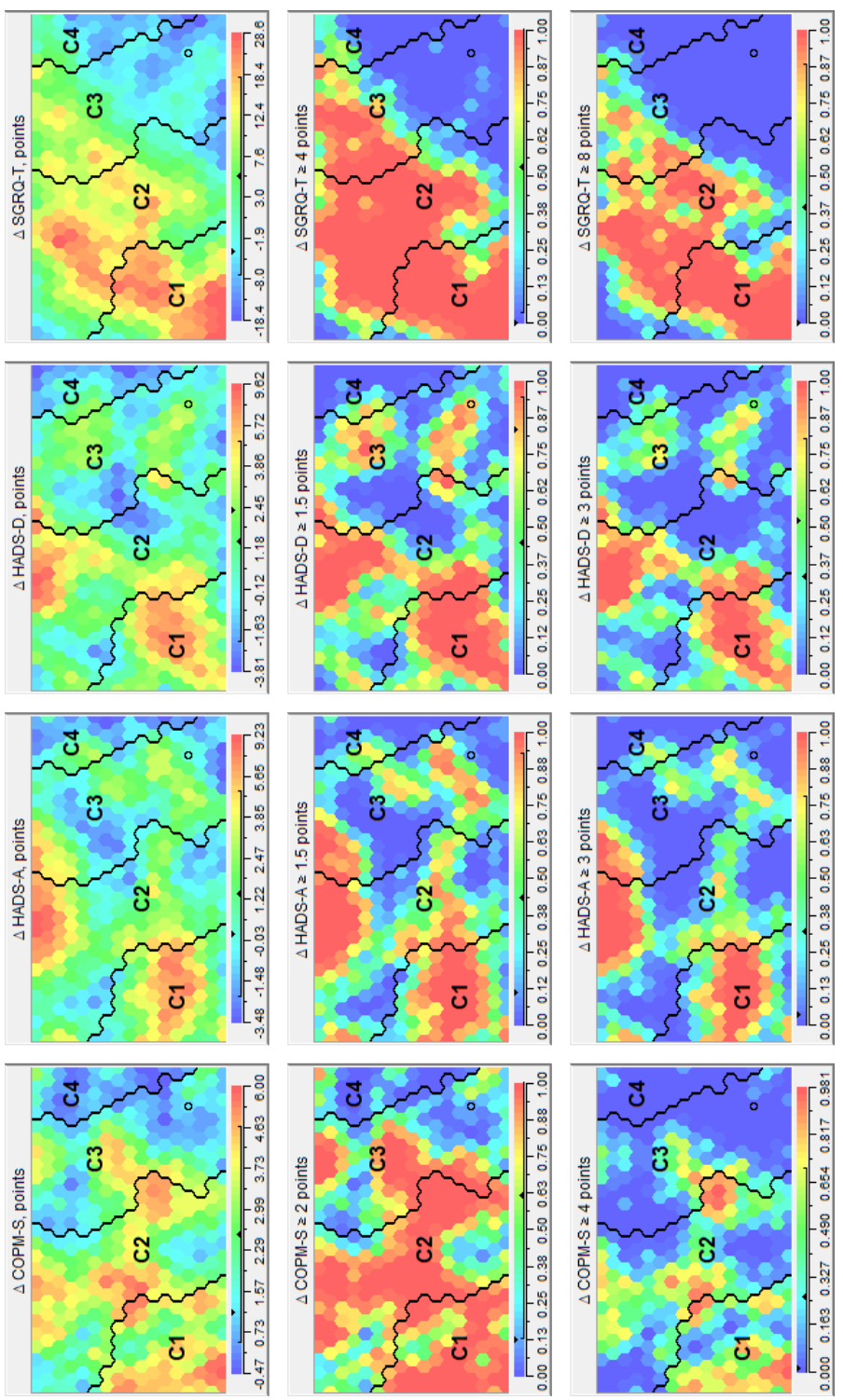

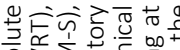

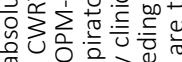

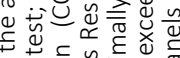

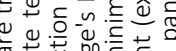

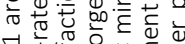

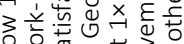

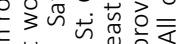

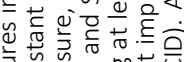

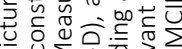

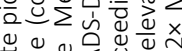

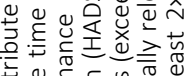
의

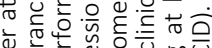

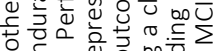

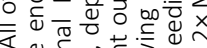
은

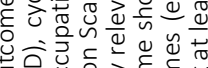

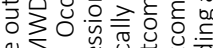

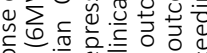

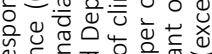

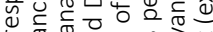
政元猔

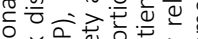

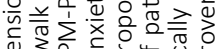

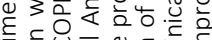

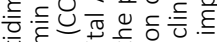

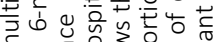

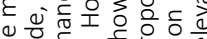

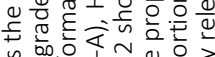

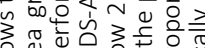

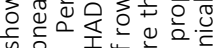

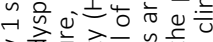
उ

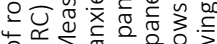

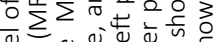

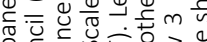

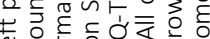

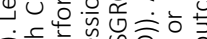

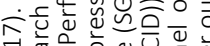

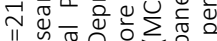

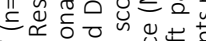

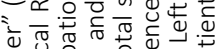

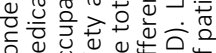

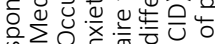

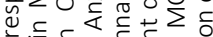

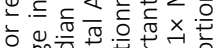

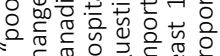

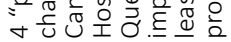


Table 5.2 Outcomes of pulmonary rehabilitation.

\begin{tabular}{|c|c|c|c|c|c|}
\hline Outcome & All patients & $\begin{array}{l}\text { Very good } \\
\text { responder }\end{array}$ & $\begin{array}{c}\text { Good } \\
\text { responder }\end{array}$ & $\begin{array}{l}\text { Moderate } \\
\text { responder }\end{array}$ & $\begin{array}{c}\text { Poor } \\
\text { responder }\end{array}$ \\
\hline Patients n (\%) & $2068(100)$ & $378(18.3)$ & 742 (35.9) & $731(35.4)$ & $217(10.5)$ \\
\hline$\triangle \mathrm{MRC}$, dyspnoea grade & $-0.4(1.1)$ & $-1.3(1.2)$ & $-0.5(1.0)^{*}$ & $-0.2(1.0) *+$ & $0.2(1.0) *+9$ \\
\hline-1 grade, $\%$ patients & 40.9 & 73.4 & $46.1 *$ & $27.9 *+$ & $17.8 *+$ \\
\hline-2 grades, $\%$ patients & 16.0 & 39.7 & $13.7^{*}$ & $11.3^{*}$ & $2.5 *+\emptyset$ \\
\hline$\triangle 6 \mathrm{MWD}, \mathrm{m}$ & $27(57)$ & $96(52)$ & $36.1(34.1)^{*}$ & $3(36) *+$ & $-48(45) *+9$ \\
\hline$\geq 30 \mathrm{~m}$ or more, $\%$ patients & 45.4 & 95.5 & $55.5 *$ & $22.3 *+$ & $1.4^{*}+\emptyset$ \\
\hline$\geq 60 \mathrm{~m}$ or more, $\%$ patients & 23.2 & 74.7 & $23.0 *$ & $3.5 *+$ & $0.0 *+\emptyset$ \\
\hline$\triangle C W R T, \mathrm{~s}$ & $208(328)$ & $525(326)$ & $290(313) *$ & $39(193) *+$ & $-17(222) *+9$ \\
\hline$\geq 100$ s or more, $\%$ patients & 51.9 & 87.7 & $68.5 *$ & $27.9 *+$ & $17.9 *+9$ \\
\hline$\geq 200$ s or more, $\%$ patients & 37.1 & 79.1 & $48.9 *$ & $12.9 *+$ & $10.5 *+$ \\
\hline$\triangle \mathrm{COPM}-\mathrm{P}$, points & $2.0(1.7)$ & $3.3(1.5)$ & $2.3(1.4)^{*}$ & $1.3(1.4) *+$ & $0.4(1.2) *+9$ \\
\hline$\geq 2$ points or more, $\%$ patients & 49.8 & 81.8 & $61.3 *$ & $32.7 *+$ & $10.5 *+9$ \\
\hline$\geq 4$ points or more, $\%$ patients & 12.8 & 36.2 & $13.7^{*}$ & $3.3 *+$ & $0.5 *+$ \\
\hline$\triangle \mathrm{COPM}-\mathrm{S}$, points & $2.6(2.1)$ & $4.1(1.9)$ & $3.1(1.8)^{*}$ & $1.8(1.7) *+$ & $0.5(1.6) *+9$ \\
\hline$\geq 2$ points or more, $\%$ patients & 61.6 & 88.8 & $74.2 *$ & $47.7 *+$ & $16.8 *+9$ \\
\hline$\geq 4$ points or more, $\%$ patients & 26.2 & 53.4 & $33.0 *$ & $11.8 *+$ & $2.1 *+\emptyset$ \\
\hline$\triangle$ HADS-A, points & $-1.4(3.5)$ & $-3.2(3.6)$ & $-1.9(3.4) *$ & $-0.7(3.1) *+$ & $1.3(2.8) *+9$ \\
\hline$\geq-1.5$ points or more, $\%$ patients & 43.5 & 65.0 & $48.8 *$ & $35.6 *+$ & $13.4 *+9$ \\
\hline$\geq-3.0$ points or more, $\%$ patients & 31.8 & 53.0 & $35.5 *$ & $24.1 *+$ & $7.0 *+9$ \\
\hline$\triangle H A D S-D$, points & $-1.4(3.5)$ & $-3.4(3.5)$ & $-2.1(3.4) *$ & $-0.5(2.9) *+$ & $1.6(2.8) *+9$ \\
\hline$\geq-1.5$ points or more, $\%$ patients & 44.8 & 69.9 & $52.1 *$ & $34.6 *+$ & $9.1 *+\emptyset$ \\
\hline$\geq-3.0$ points or more, $\%$ patients & 33.3 & 58.2 & $39.8 *$ & $21.3 *+$ & $6.4^{*}+\emptyset$ \\
\hline$\triangle S G R Q-T$, points & $-5.3(12.6)$ & $-16.0(12.7)$ & $-7.9(10.2) *$ & $-0.4(10.7) *+$ & $5.3(9.0) *+9$ \\
\hline$\geq-4$ points or more, $\%$ patients & 53.6 & 84.1 & $66.5 *$ & $36.1 *+$ & $14.9 *+9$ \\
\hline$\geq-8$ points or more, $\%$ patients & 39.7 & 74.1 & $49.5 *$ & $22.3 *+$ & $4.8 *+\emptyset$ \\
\hline
\end{tabular}

Data are presented as means (SD), unless otherwise stated. $\Delta$, change; MRC, Medical Research Council dyspnea grade; 6MWD, 6-min walk distance; CWRT, constant work-rate test; COPM-P, Canadian Occupational Performance Measure, performance score; COPM-S, Canadian Occupational Performance Measure, satisfaction score; HADS-A, Hospital Anxiety and Depression Scale, anxiety scores; HADS-D- Hospital Anxiety and Depression Scale, depression scores; SGRQ-T, St. George's Respiratory Questionnaire, total score. Missing data: MRC, $n=988 ; 6 M W D, n=107$; CWRT, $n=279$; COPM-P, $n=228$; COPM-S, $n=228$; HADS-A, $n=240 ;$ HADS-D, $\mathrm{n}=240$; SGRQ-T, $\mathrm{n}=449$. *, $\mathrm{p}<0.01$ versus cluster 'Very good responder';,$+ \mathrm{p}<0.01$ versus cluster 'Good responder'; १, $\mathrm{p}<0.01$ versus cluster 'Moderate responder'.

\section{Multidimensional response profiling, after stratification for sex and use of long-term oxygen therapy}

The current sample consisted of 1012 male and 740 female COPD patients without longterm oxygen therapy, and 168 male and 148 female COPD patients with long-term oxygen therapy. These four subgroups were also divided up into four clusters with distinct multidimensional response profiles (see Supplemental Tables S5.2 to S5.5 and Figures S5.1 to S5.4 for all details). The response to PR was best in the very good responder cluster on all outcome measures compared with the other clusters, irrespective of sex and the use of long-term oxygen therapy. 
a.

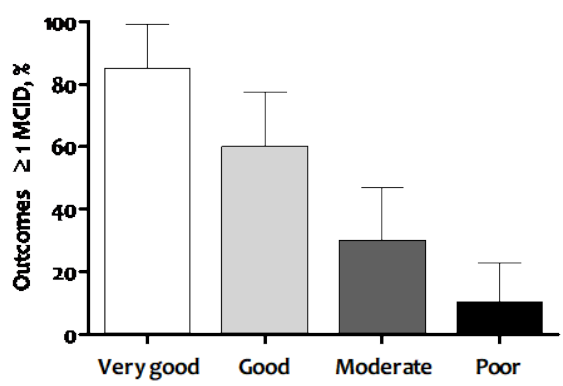

b.

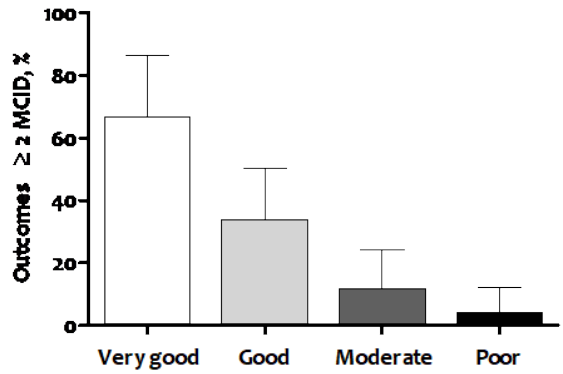

C.

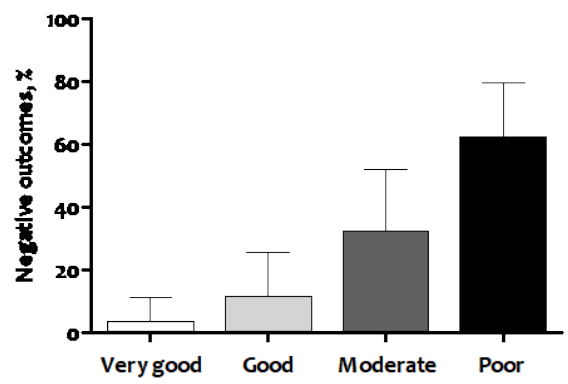

Figure 5.2 Proportion of outcomes which exceed the pre-defined minimal clinically important difference (MCID) at least a) once or b) twice, or c) have negative outcomes, in the very good, good, moderate and poor responders. 
Table 5.3 Baseline characteristics after stratification for multidimensional response clusters.

\begin{tabular}{|c|c|c|c|c|}
\hline Baseline & $\begin{array}{l}\text { Very good } \\
\text { responder }\end{array}$ & $\begin{array}{c}\text { Good } \\
\text { responder }\end{array}$ & $\begin{array}{l}\text { Moderate } \\
\text { responder }\end{array}$ & $\begin{array}{c}\text { Poor } \\
\text { responder }\end{array}$ \\
\hline Patients n (\%) & $378(18.3)$ & $742(35.9)$ & $731(35.4)$ & $217(10.5)$ \\
\hline Age, years & $62.9(8.8)$ & $63.7(9.0)$ & $64.2(8.7)$ & $64.4(9.1)$ \\
\hline Sex, \% women & 41.8 & 43.9 & 42.7 & 42.4 \\
\hline $\mathrm{FEV}_{1}, \mathrm{I}$ & $1.31(0.64)$ & $1.31(0.54)$ & $1.31(0.57)$ & $1.27(0.56)$ \\
\hline $\mathrm{FEV}_{1}, \%$ predicted & $47.4(20.2)$ & $48.9(17.8)$ & $48.8(18.3)$ & $47.9(18.8)$ \\
\hline $\mathrm{KCO}, \%$ predicted & $67.7(22.7)$ & $67.0(23.8)$ & $64.9(21.9)$ & $64.1(22.2)$ \\
\hline LTOT use, \% pts & 21.7 & 15.9 & $12.2 *$ & $12.4^{*}$ \\
\hline $\mathrm{PaO}_{2}, \mathrm{kPa}$ & $9.6(1.4)$ & $9.7(1.4)$ & $9.6(1.3)$ & $9.7(1.3)$ \\
\hline $\mathrm{PaCO}_{2}, \mathrm{kPa}$ & $5.2(0.7)$ & $5.2(0.6)$ & $5.2(0.6)$ & $5.3(0.8)$ \\
\hline $\mathrm{SaO}_{2}, \%$ & $94.9(2.6)$ & $95.0(2.4)$ & $95.1(2.1)$ & $95.0(2.1)$ \\
\hline MRC, grade & $3.7(1.1)$ & $3.3(1.1)^{*}$ & $3.2(1.1)^{*}$ & $3.2(1.1) *$ \\
\hline Exacerbation $<12 \mathrm{~m}, \mathrm{n}$ & $2.5(2.6)$ & $2.1(2.5)$ & $2.0(2.4) *$ & $2.0(1.9)$ \\
\hline Admission < $12 \mathrm{~m}, \mathrm{n}$ & $1.1(1.8)$ & $0.7(1.2) *$ & $0.6(1.3) *$ & $0.7(1.3) *$ \\
\hline CC index, points & $1.4(1.2)$ & $1.4(1.2)$ & $1.4(1.1)$ & $1.4(1.1)$ \\
\hline $\mathrm{BMI}, \mathrm{kg} / \mathrm{m}^{2}$ & $26.3(5.6)$ & $25.9(5.5)$ & $25.1(5.0)^{*+}$ & $24.8(4.6)^{*+}$ \\
\hline FFMI, $\mathrm{kg} / \mathrm{m}^{2}$ & $17.1(2.7)$ & $16.8(2.4)$ & $16.6(2.3)^{*}$ & $16.5(2.2)^{*}$ \\
\hline 6MWD, m & $405(123)$ & $452(113)^{*}$ & $461(112) *$ & $457(104) *$ \\
\hline $6 \mathrm{MWD}, \%$ predicted & $63.3(17.4)$ & $71.4(15.6)^{*}$ & $72.3(16.0) *$ & $71.7(15.7)^{*}$ \\
\hline PWR, watts & $68.2(32.3)$ & $73.5(31.4)$ & $72.9(30.5)$ & $70.4(28.3)$ \\
\hline PWR, \% predicted & $50.5(22.7)$ & $59.1(27.0)^{*}$ & $57.7(24.3) *$ & $57.3(26.3)$ * \\
\hline $\mathrm{VO}_{2}, \%$ predicted & $64.2(24.6)$ & $70.5(32.7)$ & $68.3(31.1)$ & $69.8(34.1)$ \\
\hline Ventilation, \%MVV & $84.3(22.3)$ & $84.0(21.2)$ & $83.9(20.8)$ & $87.2(22.6)$ \\
\hline CWRT, s & $295(173)$ & $320(225)$ & $326(265)$ & $296(238)$ \\
\hline COPM-P, points & $3.8(1.3)$ & $4.2(1.3) *$ & $4.5(1.3)^{*+}$ & $4.5(1.4) *+$ \\
\hline COPM-S, points & $3.2(1.6)$ & $3.6(1.7) *$ & $4.0(1.7)^{*+}$ & $4.1(1.8) *+$ \\
\hline HADS-A, points & $8.4(4.3)$ & $7.2(4.2) *$ & $6.8(4.3) *$ & $6.3(4.3) *+$ \\
\hline$\geq 8$ points, $\%$ patients & 57.0 & $45.0 *$ & $38.0 *+$ & $36.0 *$ \\
\hline HADS-D, points & $8.0(4.1)$ & $6.7(4.0) *$ & $6.4(4.0) *$ & $5.9(3.9) *+$ \\
\hline$\geq 8$ points, $\%$ patients & 55.0 & $40.0 *$ & $36.0 *$ & $32.0 *$ \\
\hline SGRQ, points & $61.5(15.2)$ & $53.6(16.5)^{*}$ & $50.2(17.1) *+$ & $50.4(17.0) *$ \\
\hline BODE index, points & $4.0(2.3)$ & $3.4(2.1)^{*}$ & $3.3(2.1) *$ & $3.4(2.0) *$ \\
\hline ADO index, points & $4.7(1.8)$ & $4.3(1.8) *$ & $4.3(1.6) *$ & $4.4(1.7)$ \\
\hline Inpatient/outpatient, \% & $64 / 36$ & $41 / 59 *$ & $31 / 69 *+$ & $25 / 75 *+$ \\
\hline
\end{tabular}

Data are presented as mean (SD), unless otherwise stated. $\mathrm{FEV}_{1}$, forced expiratory volume in $1 \mathrm{~s}$; $\mathrm{KCO}$, transfer factor of the lung for carbon monoxide; $\mathrm{PaO}_{2}$, arterial oxygen tension; $\mathrm{PaCO}_{2}$, arterial carbon dioxide tension; $\mathrm{SaO}_{2}$, arterial oxygen saturation; MRC, Medical Research Council dyspnea grade; CC index, Charlson Comorbidity Index; BMI, body mass index; FFMI, fat-free Mass Index; 6MWD, 6-min walk distance; PWR, peak work rate; $\mathrm{VO}_{2}$, oxygen uptake; $\mathrm{MVV}$, maximal voluntary ventilation; CWRT, constant work-rate test; COPM-P, Canadian Occupational Performance Measure, performance score; COPM-S, Canadian Occupational Performance Measure, satisfaction score; HADS-A, Hospital Anxiety and Depression Scale, anxiety scores; HADS-D Hospital Anxiety and Depression Scale, depression scores; SGRQ-T, St. George's Respiratory Questionnaire, total score; BODE, body mass index, airflow obstruction, dyspnoea, exercise capacity; ADO, age, dyspnoea, airflow obstruction. ${ }^{*}, p<0.01$ versus cluster very good responder; ${ }^{+}, p<0.01$ versus cluster good responder. No statistically significant differences in baseline characteristics between cluster moderate responder and cluster poor responder.

\section{Cluster characteristics after stratification for sex and use of long-term oxygen therapy}

Tables S5.6 to S5.9 in the Supplemental material summarize the baseline characteristics of the four clusters of each subgroup. In brief, in the male patients without long-term 
oxygen therapy (Table S5.6), baseline scores for problematic activities of daily life, symptoms of anxiety and depression, and health status were significantly worse in the very good responder cluster compared to the other clusters. The degree of dyspnea and the 6MWD was significantly worse in the patients of the very good responder cluster compared with the good responder or moderate responder clusters, but were comparable to values of the patients of the poor responder cluster.

In the female patients without long-term oxygen therapy (Table S5.7), the patients in the very good response cluster had a significantly higher baseline degree of dyspnea, and worse health status compared with the other three clusters. Moreover, baseline 6MWD was lower compared with moderate response and poor response clusters.

In the male patients with long-term oxygen therapy (Table S5.8), the patients in the poor response cluster had a significantly lower body mass index and fat-free mass index compared with the other three clusters. Moreover, the patients in the very good response cluster had a significantly worse health status compared to the patients of the moderate response and poor response clusters. In the female COPD patients with longterm oxygen therapy (Table S5.9), baseline 6-min walk distance was significantly lower in the patients of the very good response cluster compared with the other three clusters. Moreover, the patients in the poor response cluster had a significantly better satisfaction scores for the problematic activities of daily life compared to the other three clusters.

\section{Discussion}

The current findings corroborate that responses to regular PR outcomes are differential in a large sample of patients with COPD. Moreover, this is the first study to show that patients with COPD can be clustered based on their multidimensional response to a comprehensive PR program, identifying groups of patients with a very good, good, moderate or poor response.

\section{Differential response to PR}

Generally, PR is beneficial for adults with chronic respiratory disease, including COPD. ${ }^{1}$ Nevertheless, the response to PR may vary considerably between patients with COPD. 5,8,9,14,30,31 Moreover, individual patients respond differential on various types of outcome measures.8,11 The current results in a convenience sample of 2068 wellcharacterized patients with COPD corroborate these findings (Figure 5.1). These findings emphasize that key performance measures to evaluate the efficacy of PR in patients with COPD have to be chosen very carefully and should focus on multiple domains. The choice for exercise performance and/or health status as key performance measure is too 
simple. Indeed, to better understand the response to PR in patients with COPD, nonlinear statistics were needed. The unbiased approach to cluster patients based on their multidimensional response in a large sample of well-characterized patients with COPD is a major strength of the current analyses. Indeed, the use of Viscovery SOMs allows detailed insight in the differential responses to PR (Figure 5.1). This is a true novelty of the current analyses.

\section{Clinically relevant improvements}

In the very good responder cluster, the minimal clinically relevant improvement was achieved in $85 \%$ of the outcomes at least once and, in $67 \%$ of the outcomes, at least twice (Table 5.2; Figure 5.1). These findings suggest that the patients with COPD in the very good responder cluster are truly benefiting from PR, on (almost) all domains. Moreover, patients in the other clusters also still achieved a clinically relevant improvement in 60\% (good responder cluster), 30\% (moderate responder cluster) and $11 \%$ of the outcomes (poor responder cluster) (Table 5.2). These findings prove again the clinical value of PR in patients with COPD who are still symptomatic even though they did receive the optimal medical care before enrolment. The fact that patients in the poor response cluster still have some clinically relevant improvements in individual outcomes also confirms, that we have to be very careful in defining key outcome measures of PR.

\section{Poor response to pulmonary rehabilitation}

The poor response in a subgroup of patients with COPD (Figure 5.2) may be surprising at first sight, as PR provides a comprehensive approach. Then again, the heterogeneity in pulmonary and extra-pulmonary features ${ }^{32-34}$ is a clinical challenge to personalize PR programs for patients with COPD. Consequently, not all patients with COPD are expected to benefit from PR. $5,8,9,14,30,31$ These are clinically relevant observations, as patients, members from their social circle, health care professionals, policy makers and payers have a clear interest in the cost-effectiveness of interventions related to the integrated care of patients with COPD. So, to provide true transparency to its main stakeholders, PR services need to give detailed insights in the efficacy of pulmonary rehabilitation on the individual outcome measures, as well as in a multidimensional outcome measure.

The poor response to rehabilitation in a subgroup of patients does not seem to be COPD-specific and/or rehabilitation-specific. Indeed, also subgroups of patients with chronic neurological diseases ${ }^{35}$, chronic cardiac diseases ${ }^{36}$, or chronic musculoskeletal diseases ${ }^{37}$ respond poorly to specialized rehabilitative interventions. Moreover, response to pharmacological therapy ${ }^{38,39}$, ambulatory oxygen therapy ${ }^{40}$, bronchoscopic interventions ${ }^{41}$ and lung volume reduction surgery ${ }^{42}$ is also poor in subgroups of patients with COPD. These findings emphasize the need for a personalized approach of patients 
with chronic conditions, and the awareness that a "one size fits all" approach will not result in optimal chronic disease management. ${ }^{43}$

\section{Response prediction}

It was beyond the aim of the current study to predict response based on the baseline characteristics. Nevertheless, it seems difficult to predict at the start of the program who will end up in which cluster, as only $22.2 \%, 6.5 \%$ or $2.8 \%$ of the baseline values differed significantly $(p<0.01)$ between the poor responder cluster and the very good responder, good responder and moderate responder clusters, respectively (Tables S5.6 to S5.9). The analyses do emphasize that sex, age and the degree of airflow limitation cannot be used to identify possible responders (or non-responders) to PR as these were comparable between response clusters. Moreover, the baseline mean Charlson comorbidity score was comparable between the response clusters (Table 5.3). These findings suggest that self-reported comorbidities generally do not influence the multidimensional response to PR. Recently, Mesquita et al. ${ }^{44}$ also showed that changes in exercise performance and health status were not affected by comorbidities that were based on objective measurements.

\section{Methodological considerations}

The PR program at Ciro is executed according to the 2013 ATS/ERS Statement on Pulmonary Rehabilitation ${ }^{1}$, and provided by a skilled and dedicated team. Nevertheless, individual programs most probably varied between patients based on the results of the initial assessment. ${ }^{16}$ Indeed, the key to success may, at least in part, be hidden in the actual content of the PR program. Detailed information on the exact content of the individual program is lacking in the current study. Therefore, the present results are hypothesis-generating rather than definitive. Interestingly, the proportion of outpatients increased while the multidimensional response to PR worsened, in particular in the patients without long-term oxygen therapy (Tables S5.6 and S5.7). Whether and to what extent these differences are due to the PR setting (inpatient versus outpatient) and/or the frequency of the program ( 5 days per week for 8 weeks versus 3 days per week for 8 weeks followed by 2 days per week for 8 weeks) remains to be determined in a randomized controlled trial.

The current multidimensional response profiling was based on eight outcome measures, including two types of functional exercise performance, health status, mood status, a situational measure of dyspnea, and problematic activities of daily life, which were identified by health care professionals as essential. ${ }^{4}$ Obviously, other PR outcome measures, such as physical activity, self-efficacy and disease-specific knowledge, may also be of interest for patients with COPD. ${ }^{1}$ The current statistical approach allows the addition of other outcome measures to the multidimensional response profiling. Future, 
prospective studies need to apply their own multidimensional response profiling and, in turn, should try to corroborate the current cluster findings. Obviously, to enable concise statistical analyses at any point in time, the entire PR process (e.g., referral, baseline assessment, the rehabilitative interventions, short-term outcome assessment and follow up) must be managed and monitored by appropriate information and computer technology infrastructure. Indeed, it may even be a critical success factor for chronic disease management in general, and PR in particular.

To conclude, the current study is the first to profile the multidimensional response to PR using a non-parametric regression technique. The current approach allows us to cluster patients with COPD into groups, and, in turn, identify who benefits most or least from PR after completion of the program. For the poor responders, we may need to redesign ongoing PR programs. The current results are the next step in providing detailed insights in the performance metrics of PR in patients with COPD and the future optimization of the impact of PR. Health care professionals and payers need to start realizing that patients with COPD will respond differentially on the PR outcome measures that are regularly used. ${ }^{1}$ Choosing only one or two outcomes as key performance indicators (e.g., exercise performance and health status) seems to ignore the clinical complexity of rehabilitating patients with COPD. The time has come to start using multidimensional outcome profiling to identify the right COPD patients for the right PR program. 


\section{References}

1. Spruit MA, Singh SJ, Garvey C, et al. An official American Thoracic Society/European Respiratory Society statement: key concepts and advances in pulmonary rehabilitation. Am J Respir Crit Care Med. 2013; 188:e13-e64.

2. Lacasse $\mathrm{Y}$, Goldstein R, Lasserson TJ, et al. Pulmonary rehabilitation for chronic obstructive pulmonary disease. Cochrane Database Syst Rev. 2006;4:CD003793.

3. Nici L, ZuWallack R. An official American Thoracic Society workshop report: the Integrated Care of The COPD Patient. Proc Am Thorac Soc. 2012;9:9-18.

4. Spruit MA, Pitta F, Garvey C, et al. Differences in content and organisational aspects of pulmonary rehabilitation programmes. Eur Respir J. 2014;43:1326-37.

5. Troosters T, Gosselink R, Decramer M. Exercise training in COPD: how to distinguish responders from nonresponders. J Cardiopulm Rehabil. 2001;21:10-7.

6. Walsh JR, McKeough ZJ, Morris NR, et al. Metabolic disease and participant age are independent predictors of response to pulmonary rehabilitation. J Cardiopulm Rehabil Prev. 2013;33:249-56.

7. Scott AS, Baltzan MA, Fox J, et al. Success in pulmonary rehabilitation in patients with chronic obstructive pulmonary disease. Can Respir J. 2010;17:219-23.

8. de Torres JP, Pinto-Plata V, Ingenito E, et al. Power of outcome measurements to detect clinically significant changes in pulmonary rehabilitation of patients with COPD. Chest. 2002;121:1092-8.

9. Garrod R, Marshall J, Barley E, et al. Predictors of success and failure in pulmonary rehabilitation. Eur Respir J. 2006;27:788-94.

10. Garrod R, Ford K, Daly C, et al. Pulmonary rehabilitation: analysis of a clinical service. Physiother Res Int. 2004;9:111-20.

11. Crisafulli E, Costi S, Luppi F, et al. Role of comorbidities in a cohort of patients with COPD undergoing pulmonary rehabilitation. Thorax. 2008;63:487-92.

12. Harrison SL, Greening NJ, Williams JE, et al. Have we underestimated the efficacy of pulmonary rehabilitation in improving mood? Respir Med. 2012;106:838-44.

13. Laviolette L, Bourbeau J, Bernard S, et al. Assessing the impact of pulmonary rehabilitation on functional status in COPD. Thorax. 2008;63:115-21.

14. Spruit MA, Gosselink R, Troosters T, et al. Low-grade systemic inflammation and the response to exercise training in patients with advanced COPD. Chest. 2005;128:3183-90.

15. Sewell L, Singh SJ, Williams JE, et al. Can individualized rehabilitation improve functional independence in elderly patients with COPD? Chest. 2005;128:1194-200.

16. Spruit MA, Vanderhoven-Augustin I, Janssen PP, et al. Integration of pulmonary rehabilitation in COPD. Lancet. 2008;371:12-3.

17. van't Hul A, Gosselink R, Kwakkel G. Constant-load cycle endurance performance: test-retest reliability and validity in patients with COPD. J Cardiopulm Rehabil. 2003;23:143-50.

18. Hernandes NA, Wouters EF, Meijer K, et al. Reproducibility of 6-minute walking test in patients with COPD. Eur Respir J. 2011;38:261-7.

19. Holland AE, Spruit MA, Troosters T, et al. An official European Respiratory Society/American Thoracic Society technical standard: field walking tests in chronic respiratory disease. Eur Respir J. 2014;44: 1428-46.

20. Singh SJ, Puhan MA, Andrianopoulos V, et al. An official systematic review of the European Respiratory Society/ American Thoracic Society: measurement properties of field walking tests in chronic respiratory disease. Eur Respir J. 2014;44:1447-78.

21. Annegarn J, Meijer K, Passos VL, et al. Problematic activities of daily life are weakly associated with clinical characteristics in COPD. J Am Med Dir Assoc. 2012;13:284-90.

22. Sewell L, Singh SJ. The Canadian Occupational Performance Measure: is it a reliable measure in clients with chronic obstructive pulmonary disease? Br J Occup Ther. 2001;64:305-10.

23. Zigmond AS, Snaith RP. The hospital anxiety and depression scale. Acta Psychiatr Scand. 1983;67: 361-70.

24. Jones PW, Quirk FH, Baveystock CM, et al. A self-complete measure of health status for chronic airflow limitation. The St. George's Respiratory Questionnaire. Am Rev Respir Dis. 1992;145:1321-7. 
25. Vanfleteren LE, Spruit MA, Groenen MT, et al. Arterial stiffness in patients with COPD: the role of systemic inflammation and the effects of pulmonary rehabilitation. Eur Respir J. 2014;43:1306-15.

26. Sillen MJ, Franssen FM, Delbressine JM, et al. Efficacy of lower-limb muscle training modalities in severely dyspnoeic individuals with COPD and quadriceps muscle weakness: results from the DICES trial. Thorax. 2014;69:525-31.

27. Vanfleteren LE, Spruit MA, Groenen M, et al. Clusters of comorbidities based on validated objective measurements and systemic inflammation in patients with chronic obstructive pulmonary disease. Am J Respir Crit Care Med. 2013;187:728-35.

28. Puhan MA, Frey M, Buchi S, et al. The minimal important difference of the hospital anxiety and depression scale in patients with chronic obstructive pulmonary disease. Health Qual Life Outcomes. 2008;6:46.

29. Jones PW. Interpreting thresholds for a clinically significant change in health status in asthma and COPD. Eur Respir J. 2002;19:398-404.

30. Ries AL, Make BJ, Lee SM, et al. The effects of pulmonary rehabilitation in the national emphysema treatment trial. Chest. 2005;128:3799-809.

31. Spruit MA, Gosselink R, Troosters T, et al. Resistance versus endurance training in patients with COPD and peripheral muscle weakness. Eur Respir J. 2002;19:1072-8.

32. Sillen MJ, Franssen FM, Delbressine JM, et al. Heterogeneity in clinical characteristics and co-morbidities in dyspneic individuals with COPD GOLD D: findings of the DICES trial. Respir Med. 2013; 107:1186-94.

33. Spruit MA, Pennings HJ, Janssen PP, et al. Extra-pulmonary features in COPD patients entering rehabilitation after stratification for MRC dyspnea grade. Respir Med. 2007;101:2454-63.

34. Agusti A, Calverley PM, Celli B, et al. Characterisation of COPD heterogeneity in the ECLIPSE cohort. Respir Res. 2010;11:122.

35. Bowden MG, Behrman AL, Neptune RR, et al. Locomotor rehabilitation of individuals with chronic stroke: difference between responders and nonresponders. Arch Phys Med Rehabil. 2013;94:856-62.

36. Schmid JP, Zurek M, Saner H. Chronotropic incompetence predicts impaired response to exercise training in heart failure patients with sinus rhythm. Eur J Prevent Cardiol. 2013;20:585-92.

37. Cecchi F, Pasquini G, Paperini A, et al. Predictors of response to exercise therapy for chronic low back pain: result of a prospective study with one year follow-up. Eur J Phys Rehabil Med. 2014;50:143-51.

38. Jones PW, Donohue JF, Nedelman J, et al. Correlating changes in lung function with patient outcomes in chronic obstructive pulmonary disease: a pooled analysis. Respir Res. 2011;12:161.

39. Burge PS, Calverley PM, Jones PW, et al. Prednisolone response in patients with chronic obstructive pulmonary disease: results from the ISOLDE study. Thorax. 2003;58:654-8.

40. Nonoyama ML, Brooks D, Guyatt GH, et al. Effect of oxygen on health quality of life in patients with chronic obstructive pulmonary disease with transient exertional hypoxemia. Am J Respir Crit Care Med. 2007;176:343-9.

41. Deslee G, Klooster K, Hetzel M, et al. Lung volume reduction coil treatment for patients with severe emphysema: a European multicentre trial. Thorax. 2014;69:980-6.

42. Fishman A, Martinez F, Naunheim K, et al. A randomized trial comparing lung-volume-reduction surgery with medical therapy for severe emphysema. N Engl J Med. 2003;348:2059-73.

43. Flores M, Glusman G, Brogaard K, et al. P4 medicine: how systems medicine will transform the healthcare sector and society. Per Med. 2013;10:565-76.

44. Mesquita R, Vanfleteren LE, Franssen FME, et al. Objectively identified comorbidities in COPD: impact on pulmonary rehabilitation outcomes. Eur Respir J. 2015;46:545-8. 


\section{Supplemental material}

Table S5.1 Overview of pulmonary and non-pulmonary drug treatments.

\begin{tabular}{|c|c|c|c|c|c|}
\hline & $\begin{array}{c}\text { All } \\
\text { patients }\end{array}$ & $\begin{array}{c}\text { Men } \\
\text { without } \\
\text { LTOT }\end{array}$ & $\begin{array}{c}\text { Women } \\
\text { without } \\
\text { LTOT }\end{array}$ & $\begin{array}{l}\text { Men } \\
\text { with } \\
\text { LTOT }\end{array}$ & $\begin{array}{c}\text { Women } \\
\text { with } \\
\text { LTOT }\end{array}$ \\
\hline Patients n (\%) & $2068(100)$ & 1012 (48.9) & $740(35.8)$ & $168(8.1)$ & $148(7.2)$ \\
\hline Short acting $\beta 2$-agonists (SABA), \% patients & 36.2 & 31.4 & 40.5 & 41.8 & 40.9 \\
\hline Short-acting anticholinergics (SAMA), \% patients & 11.1 & 10.6 & 10.8 & 11.5 & 15.5 \\
\hline SABA/SAMA combination, $\%$ patients & 23.1 & 20.4 & 17.6 & 45.5 & 43.6 \\
\hline Long-acting $\beta 2$-agonists (LABA) , \% patients & 31.2 & 31.6 & 33.0 & 26.1 & 25.4 \\
\hline Long-acting anticholinergics (LAMA), \% patients & 75.1 & 74.6 & 76.8 & 73.3 & 71.8 \\
\hline Inhaled corticosteroids (ICS), \% patients & 17.4 & 15.7 & 18.0 & 23.6 & 19.0 \\
\hline ICS/LABA combination, $\%$ patients & 61.9 & 61.9 & 60.6 & 63.6 & 66.2 \\
\hline Theolair, \% patients & 14.5 & 13.6 & 10.0 & 27.3 & 29.6 \\
\hline Oral corticosteroids, \% patients & 16.7 & 12.6 & 11.6 & 49.1 & 33.8 \\
\hline ACE/ARB, $\%$ patients & 26.1 & 29.7 & 23.2 & 21.8 & 21.1 \\
\hline Beta-blockers, \% patients & 16.7 & 19.3 & 13.9 & 18.2 & 10.6 \\
\hline Calcium blockers, $\%$ patients & 15.0 & 15.6 & 12.9 & 21.8 & 12.7 \\
\hline Anti-arrythmica, \% patients & 5.2 & 6.6 & 2.3 & 10.3 & 4.2 \\
\hline Nitrates, $\%$ patients & 9.9 & 11.9 & 6.3 & 14.6 & 9.2 \\
\hline Diuretics, \% patients & 25.2 & 23.7 & 20.3 & 39.4 & 44.4 \\
\hline Anti-limpaemica, \% patients & 26.8 & 32.0 & 20.2 & 29.1 & 21.8 \\
\hline Ant-aggregates, \% patients & 26.6 & 33.7 & 17.0 & 31.5 & 20.4 \\
\hline Coumarines, \% patients & 7.9 & 8.7 & 6.1 & 10.3 & 8.5 \\
\hline Oral anti-diabetica/insulin, \% patients & 8.1 & 9.6 & 3.7 & 17.6 & 9.9 \\
\hline Calcium and/or vitamin D supplements, \% patients & 13.6 & 9.4 & 14.2 & 23.0 & 28.9 \\
\hline Bisphosphonates, \% patients & 13.9 & 9.8 & 12.5 & 26.7 & 35.2 \\
\hline Anti-depressives, \% patients & 12.2 & 8.5 & 15.8 & 16.4 & 14.8 \\
\hline Anxiolytics, \% patients & 13.9 & 7.5 & 16.9 & 20.6 & 35.9 \\
\hline Sleep medication, \% patients & 9.7 & 7.1 & 12.1 & 9.1 & 16.2 \\
\hline Paracetamol, \% patients & 6.1 & 4.5 & 6.4 & 9.1 & 12.0 \\
\hline NSAIDs, \% patients & 5.3 & 4.9 & 6.0 & 4.9 & 4.9 \\
\hline Proton-pump inhibitor, $\%$ patients & 34.8 & 31.2 & 31.1 & 55.2 & 55.6 \\
\hline Antibiotics, $\%$ patients & 7.7 & 5.3 & 5.0 & 29.7 & 12.7 \\
\hline Acetylcystein, \% patients & 28.1 & 25.2 & 21.5 & 56.4 & 50.0 \\
\hline
\end{tabular}


Table S5.2 Outcomes of pulmonary rehabilitation in 1012 male COPD patients without long-term oxygen therapy.

\begin{tabular}{|c|c|c|c|c|c|}
\hline Outcome & $\begin{array}{l}\text { Men without } \\
\text { LTOT }\end{array}$ & $\begin{array}{l}\text { Very good } \\
\text { responder }\end{array}$ & $\begin{array}{l}\text { Good } \\
\text { responder }\end{array}$ & $\begin{array}{l}\text { Moderate } \\
\text { responder }\end{array}$ & $\begin{array}{l}\text { Poor } \\
\text { responder }\end{array}$ \\
\hline Patients n (\%) & $1012(100)$ & $276(27.3)$ & $299(29.6)$ & $185(18.3)$ & $252(24.9)$ \\
\hline$\Delta \mathrm{MRC}$, grade & $-0.4(1.1)$ & $-1.2(1.1)$ & $-0.5(0.9)^{*}$ & $-0.1(0.3)^{*+}$ & $0.1(0.9)^{*+}$ \\
\hline-1 grade, $\%$ pts & 39.2 & 70.9 & $42.0^{*}$ & $23.5^{*+}$ & $17.1^{*+}$ \\
\hline-2 grades, $\%$ pts & 16.5 & 37.0 & $12.3^{*}$ & $11.8^{*}$ & $5.4^{*}$ \\
\hline$\Delta 6 \mathrm{MWD}, \mathrm{m}$ & $27(58)$ & $81(57)$ & $34(33)^{*}$ & $4(28)^{*+}$ & $-24(45)^{*+1}$ \\
\hline$\geq 30 \mathrm{~m}$ or more, $\%$ pts & 44.4 & 82.9 & $56.0 *$ & $16.9 *+$ & $9.9 *+$ \\
\hline$\geq 60 \mathrm{~m}$ or more, $\%$ pts & 22.1 & 58.4 & $19.4^{*}$ & $1.7^{*+}$ & $1.7^{*+}$ \\
\hline$\Delta \mathrm{CWRT}, \mathrm{s}$ & $229(351)$ & $537(358)$ & $250(281)^{*}$ & $10(128)^{*+}$ & $17(256)^{*+}$ \\
\hline$\geq 100$ s or more, $\%$ pts & 53.2 & 84.4 & $65.7^{*}$ & $23.3^{*+}$ & $24.5^{*+}$ \\
\hline$\geq 200$ s or more, $\%$ pts & 40.7 & 78.6 & $48.1^{*}$ & $5.7^{*+}$ & $14.8 *+9$ \\
\hline$\Delta$ COPM-P, points & $1.8(1.6)$ & $2.9(1.5)$ & $2.0(1.3)^{*}$ & $1.4(1.3)^{*+}$ & $0.6(1.1)^{*+\emptyset}$ \\
\hline$\geq 2$ points or more, $\%$ pts & 45.3 & 75.1 & $48.6^{*}$ & $37.8^{*}$ & $12.6 *+9$ \\
\hline$\geq 4$ points or more, $\%$ pts & 11.0 & 28.5 & $7.4^{*}$ & $3.0^{*}$ & $0.9 *+$ \\
\hline$\Delta$ COPM-S, points & $2.3(2.0)$ & $3.6(1.9)$ & $2.7(1.8)^{*}$ & $1.9(1.7)^{*+}$ & $0.8(1.5)^{*+9}$ \\
\hline$\geq 2$ points or more, $\%$ pts & 57.6 & 81.5 & $67.7^{*}$ & $53.7^{*+}$ & $20.9 *+\emptyset$ \\
\hline$\geq 4$ points or more, $\%$ pts & 22.2 & 41.1 & $27.2^{*}$ & $11.0 *+$ & $2.8^{*+1}$ \\
\hline$\Delta$ HADS-A, points & $-1.0(3.2)$ & $-2.6(3.3)$ & $-1.1(2.7)^{*}$ & $-1.1(3.1)^{*}$ & $0.8(2.8)^{*+1}$ \\
\hline-1.5 points or more, \% pts & 40.2 & 59.7 & $39.0 *$ & $40.4^{*}$ & $20.9 *+9$ \\
\hline-3.0 points or more, $\%$ pts & 27.9 & 44.0 & $26.5^{*}$ & $28.1^{*}$ & $2.8 *+\emptyset$ \\
\hline$\Delta$ HADS-D, points & $-1.1(3.3)$ & $-2.9(3.2)$ & $-1.2(2.9)^{*}$ & $-1.2(2.8)^{*}$ & $0.8(2.9) *+9$ \\
\hline$\geq-1.5$ points or more, $\%$ pts & 41.8 & 63.5 & $40.5^{*}$ & $41.8^{*}$ & $19.3 *+\emptyset$ \\
\hline$\geq-3.0$ points or more, $\%$ pts & 31.1 & 53.8 & $28.4^{*}$ & $31.8^{*}$ & $8.5^{*+1}$ \\
\hline$\Delta \mathrm{SGRQ}-\mathrm{T}$, points & $-5.5(12.5)$ & $-14.5(12.5)$ & $-6.0(10.3)^{*}$ & $-4.1(9.1)^{*}$ & $4.0(9.7)^{*+9}$ \\
\hline$\geq-4$ points or more, $\%$ pts & 55.2 & 80.0 & $62.7 *$ & $52.8^{*}$ & $19.8 *+\emptyset$ \\
\hline$\geq-8$ points or more, $\%$ pts & 39.7 & 66.4 & $45.5^{*}$ & $33.8^{*}$ & $7.1^{*+9}$ \\
\hline $\begin{array}{l}\text { Outcome measures with } \geq 1 x \\
\text { MCID, \% outcomes }\end{array}$ & $47.7(28.5)$ & $78.0(17.0)$ & $55.7(16.6)^{*}$ & $31.0(14.4)^{*+}$ & $17.2(16.1)^{*+9}$ \\
\hline $\begin{array}{l}\text { Outcome measures with } \geq 2 x \\
\text { MCID, } \% \text { outcomes }\end{array}$ & $28.5(25.5)$ & $58.0(21.3)$ & $29.6(16.0)^{*}$ & $12.5(11.5)^{*+}$ & $6.6(10.0)^{*+1}$ \\
\hline $\begin{array}{l}\text { Negative outcomes, \% } \\
\text { outcomes }\end{array}$ & $24.0(23.5)$ & $5.4(8.7)$ & $14.4(14.9)^{*}$ & $31.0(19.1)^{*+}$ & $50.7(19.3)^{*+1}$ \\
\hline
\end{tabular}

Data are presented as means (SD), unless otherwise stated. $\Delta$, change; MRC, Medical Research Council dyspnea grade; 6MWD, 6-min walk distance; CWRT, constant work-rate test; COPM-P, Canadian Occupational Performance Measure, performance score; COPM-S, Canadian Occupational Performance Measure, satisfaction score; HADS-A, Hospital Anxiety and Depression Scale, anxiety scores; HADS-D, Hospital Anxiety and Depression Scale, depression scores; SGRQ-T, St. George's Respiratory Questionnaire, total score; MCID, Minimal Clinically Important Difference. *, $p<0.01$ versus cluster 'Very good responder'; $\dagger$, $p<0.01$ versus cluster 'Good responder'; ๆ, $\mathrm{p}<0.01$ versus cluster 'Moderate responder'. 
Table S5.3 Outcomes of pulmonary rehabilitation in 740 female COPD patients without long-term oxygen therapy.

\begin{tabular}{|c|c|c|c|c|c|}
\hline Outcome & $\begin{array}{c}\text { Women } \\
\text { without LTOT }\end{array}$ & $\begin{array}{l}\text { Very good } \\
\text { responder }\end{array}$ & $\begin{array}{l}\text { Good } \\
\text { responder }\end{array}$ & $\begin{array}{l}\text { Moderate } \\
\text { responder }\end{array}$ & $\begin{array}{l}\text { Poor } \\
\text { responder }\end{array}$ \\
\hline Patients n (\%) & $740(100)$ & $169(22.8)$ & $260(35.1)$ & $182(24.6)$ & $129(17.4)$ \\
\hline$\Delta \mathrm{MRC}$, grade & $-0.4(1.1)$ & $-1.1(1.1)$ & $-0.4(1.0)^{*}$ & $-0.2(1.0)^{*}$ & $0.1(0.9) *+$ \\
\hline-1 grade, $\%$ pts & 41.2 & 69.9 & $40.0^{*}$ & $33.7^{*}$ & $23.9 *$ \\
\hline-2 grades, $\%$ pts & 13.9 & 31.5 & $13.3^{*}$ & $10.5^{*}$ & $1.4^{*+}$ \\
\hline$\Delta 6 \mathrm{MWD}, \mathrm{m}$ & $22(51)$ & $73(46)$ & $33(31)^{*}$ & $0(25)^{*+}$ & $-36(42) *+9$ \\
\hline$\geq 30 \mathrm{~m}$ or more, $\%$ pts & 40.7 & 83.3 & $53.5^{*}$ & $8.7^{*+}$ & $4.1^{*}+$ \\
\hline$\geq 60 \mathrm{~m}$ or more, $\%$ pts & 19.8 & 56.4 & $19.3^{*}$ & $1.2^{*+}$ & $0 *+$ \\
\hline$\Delta \mathrm{CWRT}, \mathrm{s}$ & $197(311)$ & 532 (319) & $193(236)^{*}$ & $77(171)^{*+}$ & $-44(218) *+9$ \\
\hline$\geq 100$ s or more, $\%$ pts & 53.8 & 90.3 & $61.0^{*}$ & $38.9 *+$ & $14.8 *+9$ \\
\hline$\geq 200$ s or more, $\%$ pts & 35.4 & 77.8 & $35.5^{*}$ & $18.5^{*+}$ & $6.1^{*+1}$ \\
\hline$\Delta$ COPM-P, points & $1.9(1.6)$ & $2.8(1.6)$ & $2.3(1.4)^{*}$ & $1.3(1.3)^{*+}$ & $0.6(1.3)^{*+1}$ \\
\hline$\geq 2$ points or more, $\%$ pts & 48.8 & 71.1 & 62.8 & $35.4^{*+}$ & $9.6 *+\emptyset$ \\
\hline$\geq 4$ points or more, $\%$ pts & 10.6 & 25.5 & $11.2^{*}$ & $2.4^{*}+$ & $1.7^{*+}$ \\
\hline$\Delta$ COPM-S, points & $2.6(2.1)$ & $3.8(1.9)$ & $3.1(1.9)^{*}$ & $1.9(1.6)^{*+}$ & $0.9(1.8)^{*+1}$ \\
\hline$\geq 2$ points or more, $\%$ pts & 61.6 & 81.2 & 73.1 & $50.6 *+$ & $27.8 *+9$ \\
\hline$\geq 4$ points or more, $\%$ pts & 27.2 & 51.7 & $33.5^{*}$ & $11.0^{*+}$ & $5.2 *+$ \\
\hline$\Delta$ HADS-A, points & $-1.6(3.7)$ & $-3.2(3.1)$ & $-1.8(3.9)^{*}$ & $-1.2(3.8)^{*}$ & $0.4(3.2)^{*+1}$ \\
\hline$\geq-1.5$ points or more, $\%$ pts & 47.4 & 69.6 & $48.6^{*}$ & $43.6^{*}$ & $21.4 *+\emptyset$ \\
\hline$\geq-3.0$ points or more, $\%$ pts & 35.0 & 57.4 & $32.9 *$ & $32.5^{*}$ & $13.4 *+9$ \\
\hline$\Delta$ HADS-D, points & $-1.4(3.7)$ & $-3.1(3.4)$ & $-2.0(3.6)^{*}$ & $-0.6(3.5)^{*+}$ & $0.7(3.1)^{*+9}$ \\
\hline$\geq-1.5$ points or more, $\%$ pts & 45.0 & 66.7 & $52.0 *$ & $36.4^{*+}$ & $15.7 *+\emptyset$ \\
\hline$\geq-3.0$ points or more, $\%$ pts & 32.3 & 51.7 & $38.1^{*}$ & $21.6^{*+}$ & $11.3^{*+}$ \\
\hline$\Delta \mathrm{SGRQ}-\mathrm{T}$, points & $-4.6(12.4)$ & $-14.2(11.0)$ & $-5.4(10.6)^{*}$ & $-2.2(10.8)^{*+}$ & $5.8(10.0)^{*+1}$ \\
\hline$\geq-4$ points or more, $\%$ pts & 51.2 & 81.0 & $57.3^{*}$ & $40.4^{*+}$ & $15.2 *+9$ \\
\hline$\geq-8$ points or more, $\%$ pts & 38.3 & 70.8 & $40.7^{*}$ & $28.4^{*}$ & $4.8 *+9$ \\
\hline $\begin{array}{l}\text { Outcome measures with } \geq 1 x \\
\text { MCID, \% outcomes }\end{array}$ & $47.9(28.5)$ & $80.7(16.9)$ & $56.4(17.7)^{*}$ & $30.2(14.4)^{*+}$ & $13.0(11.8)^{*+1}$ \\
\hline $\begin{array}{l}\text { Outcome measures with } \geq 2 x \\
\text { MCID, \% outcomes }\end{array}$ & $27.6(24.4)$ & $59.7(18.8)$ & $27.9(16.2)^{*}$ & $13.9(12.4)^{* \dagger}$ & $4.4(7.6)^{*+1}$ \\
\hline $\begin{array}{l}\text { Negative outcomes, \% } \\
\text { outcomes }\end{array}$ & $23.3(24.1)$ & $3.9(8.2)$ & $14.0(15.8)^{*}$ & $30.9(19.5)^{*+}$ & $56.6(18.4)^{*+9}$ \\
\hline
\end{tabular}

Data are presented as means (SD), unless otherwise stated. $\Delta$, change; MRC, Medical Research Council dyspnea grade; 6MWD, 6-min walk distance; CWRT, constant work-rate test; COPM-P, Canadian Occupational Performance Measure, performance score; COPM-S, Canadian Occupational Performance Measure, satisfaction score; HADS-A, Hospital Anxiety and Depression Scale, anxiety scores; HADS-D, Hospital Anxiety and Depression Scale, depression scores; SGRQ-T, St. George's Respiratory Questionnaire, total Score; MCID, Minimal Clinically Important Difference. *, $p<0.01$ versus cluster 'Very good responder'; $\dagger, p<0.01$ versus cluster 'Good responder'; $\mathbf{\emptyset}, \mathrm{p}<0.01$ versus cluster 'Moderate responder'. 
Table S5.4 Outcomes of pulmonary rehabilitation in 168 male COPD patients with long-term oxygen therapy.

\begin{tabular}{|c|c|c|c|c|c|}
\hline Outcome & $\begin{array}{l}\text { Men with } \\
\text { LTOT }\end{array}$ & $\begin{array}{l}\text { Very good } \\
\text { responder }\end{array}$ & $\begin{array}{l}\text { Good } \\
\text { responder }\end{array}$ & $\begin{array}{l}\text { Moderate } \\
\text { responder }\end{array}$ & $\begin{array}{c}\text { Poor } \\
\text { responder }\end{array}$ \\
\hline Patients n (\%) & $168(100)$ & $52(31.0)$ & $58(34.5)$ & $34(20.2)$ & $24(14.3)$ \\
\hline$\Delta \mathrm{MRC}$, grade & $-0.5(1.3)$ & $-1.0(1.4)$ & $-0.4(1.2)$ & $-0.3(1.1)$ & $0.2(1.0)^{*}$ \\
\hline 1 grade, $\%$ pts & 43.4 & 70.0 & $36.4^{*}$ & 36.8 & $17.6^{*}$ \\
\hline 2 grades, $\%$ pts & 19.2 & 30.0 & 21.2 & 10.5 & 5.9 \\
\hline$\Delta 6 \mathrm{MWD}, \mathrm{m}$ & $34(72)$ & $95(45)$ & $41(34)^{*}$ & $2(46)^{*+}$ & $-79(67)^{*+1}$ \\
\hline$\geq 30 \mathrm{~m}$ or more, $\%$ pts & 56.3 & 90.0 & $60.7^{*}$ & $26.7^{*+}$ & $0 *+\emptyset$ \\
\hline$\geq 60 \mathrm{~m}$ or more, $\%$ pts & 37.3 & 80.0 & $26.8^{*}$ & $6.7^{*}$ & $0 *+$ \\
\hline$\Delta \mathrm{CWRT}, \mathrm{s}$ & $163(298)$ & $398(340)$ & $143(233)^{*}$ & $32(99)^{*}$ & $-47(269)^{*+}$ \\
\hline$\geq 100$ s or more, $\%$ pts & 43.7 & 80.0 & $46.0^{*}$ & $12.5^{*+}$ & $15.0^{*}$ \\
\hline$\geq 200$ s or more, $\%$ pts & 28.2 & 70.0 & $20.0 *$ & $6.3^{*}$ & $1.0^{*}$ \\
\hline$\Delta$ COPM-P, points & $2.3(1.7)$ & $3.4(1.5)$ & $2.3(1.5)^{*}$ & $1.7(1.5)^{*}$ & $0.7(1.4)^{*+}$ \\
\hline$\geq 2$ points or more, $\%$ pts & 59.3 & 80.0 & 60.4 & $45.5^{*}$ & $26.3^{*}$ \\
\hline$\geq 4$ points or more, $\%$ pts & 17.3 & 40.0 & $13.2^{*}$ & $6.1^{*}$ & $0 *$ \\
\hline$\Delta$ COPM-S, points & $2.8(2.1)$ & $4.0(1.8)$ & $2.9(2.0)^{*}$ & $2.0(1.7)^{*}$ & $0.9(1.8)^{*+}$ \\
\hline$\geq 2$ points or more, $\%$ pts & 69.1 & 90.0 & 75.5 & $51.5^{*}$ & $22.2 *+$ \\
\hline$\geq 4$ points or more, $\%$ pts & 28.9 & 40.0 & 28.3 & 18.2 & 11.1 \\
\hline$\Delta$ HADS-A, points & $-1.9(3.8)$ & $-4.0(4.8)$ & $-2.1(2.77)$ & $-0.4(2.2)^{* \dagger}$ & $1.2(2.4)^{*+}$ \\
\hline$\geq 1.5$ points or more, $\%$ pts & 44.1 & 60.0 & 46.2 & $31.3^{*}$ & $17.6^{*}$ \\
\hline$\geq 3.0$ points or more, $\%$ pts & 35.2 & 60.0 & 38.5 & $15.6^{*}$ & $5.9 *$ \\
\hline$\Delta$ HADS-D, points & $-2.1(3.6)$ & $-3.6(4.1)$ & $-2.6(2.9)$ & $-0.4(2.6)^{*+}$ & $-0.1(3.3)^{*+}$ \\
\hline$\geq 1.5$ points or more, $\%$ pts & 56.6 & 80.0 & 61.5 & $34.4^{*}$ & $35.3^{*}$ \\
\hline$\geq 3.0$ points or more, $\%$ pts & 44.1 & 60.0 & 44.2 & $25.0^{*}$ & 35.3 \\
\hline$\Delta$ SGRQ-T, points & $-6.2(11.9)$ & $-17.0(9.5)$ & $-5.6(11.6)^{*}$ & $-1.3(8.1)^{*}$ & $3.1(8.0)^{*+}$ \\
\hline$\geq 4$ points or more, $\%$ pts & 56.3 & 100.0 & $57.1^{*}$ & $32.1^{*}$ & $22.2^{*}$ \\
\hline$\geq 8$ points or more, $\%$ pts & 43.7 & 90.0 & $44.9 *$ & $14.3^{*+}$ & $0 *+$ \\
\hline $\begin{array}{l}\text { Outcome measures with } \geq 1 x \\
\text { MCID, \% outcomes }\end{array}$ & $53.6(30.1)$ & $85.1(14.5)$ & $55.9(19.6)^{*}$ & $29.1(13.6)^{*+}$ & $14.3(12.8)^{*+1}$ \\
\hline $\begin{array}{l}\text { Outcome measures with } \geq 2 x \\
\text { MCID, \% outcomes }\end{array}$ & $34.4(28.7)$ & $68.3(17.9)$ & $29.2(16.7)^{*}$ & $11.1(11.8)^{*+}$ & $6.5(9.2)^{*+}$ \\
\hline $\begin{array}{l}\text { Negative outcomes, } \% \\
\text { outcomes }\end{array}$ & $21.4(24.0)$ & $5.9(9.4)$ & $13.3(14.7)^{*}$ & $29.7(19.6)^{*+}$ & $62.7(17.7)^{*+9}$ \\
\hline
\end{tabular}

Data are presented as means (SD), unless otherwise stated. $\Delta$, change; MRC, Medical Research Council dyspnea grade; 6MWD, 6-min walk distance; CWRT, constant work-rate test; COPM-P, Canadian Occupational Performance Measure, performance score; COPM-S, Canadian Occupational Performance Measure, satisfaction score; HADS-A, Hospital Anxiety and Depression Scale, anxiety scores; HADS-D, Hospital Anxiety and Depression Scale, depression scores; SGRQ-T, St. George's Respiratory Questionnaire, total Score; MCID, Minimal Clinically Important Difference. *, $p<0.01$ versus cluster 'Very good responder'; $\dagger, p<0.01$ versus cluster 'Good responder'; $\uparrow$, $p<0.01$ versus cluster 'Moderate responder'. 
Table S5.5 Outcomes of pulmonary rehabilitation in 148 female COPD patients with long-term oxygen therapy.

\begin{tabular}{|c|c|c|c|c|c|}
\hline Outcome & $\begin{array}{c}\text { Women with } \\
\text { LTOT }\end{array}$ & $\begin{array}{l}\text { Very good } \\
\text { responder }\end{array}$ & $\begin{array}{c}\text { Good } \\
\text { responder }\end{array}$ & $\begin{array}{l}\text { Moderate } \\
\text { responder }\end{array}$ & $\begin{array}{c}\text { Poor } \\
\text { responder }\end{array}$ \\
\hline Patients n (\%) & $148(100)$ & $33(22.3)$ & $44(29.7)$ & $42(28.4)$ & $29(19.6)$ \\
\hline$\Delta \mathrm{MRC}$, grade & $-0.5(1.1)$ & $-1.4(1.0)$ & $-0.6(0.8)^{*}$ & $-0.3(1.3) *$ & $0.1(0.8)^{*}$ \\
\hline 1 grade, $\%$ pts & 47.1 & 80.0 & 52.0 & $36.0 *$ & $17.6^{*}$ \\
\hline 2 grades, $\%$ pts & 18.4 & 55.0 & $12.0 *$ & $8.0 *$ & $0 *$ \\
\hline$\Delta 6 \mathrm{MWD}, \mathrm{m}$ & $44(63)$ & $116(63)$ & $45(23)^{*}$ & $7(51)^{*+}$ & $7(45) *+$ \\
\hline$\geq 30 \mathrm{~m}$ or more, $\%$ pts & 63.6 & 96.9 & 79.1 & $37.5^{*+}$ & $36.0 *+$ \\
\hline$\geq 60 \mathrm{~m}$ or more, $\%$ pts & $31.4+$ & 81.3 & $27.9 *$ & $10.0^{*}$ & $8.0 *$ \\
\hline$\Delta \mathrm{CWRT}, \mathrm{s}$ & $156(246)$ & 437 (305) & $167(196)^{*}$ & $82(94)^{*}$ & $-11(177)^{*+}$ \\
\hline$\geq 100$ s or more, $\%$ pts & 41.1 & 86.4 & $43.8^{*}$ & $29.4^{*}$ & $12.5^{*}$ \\
\hline$\geq 200$ s or more, $\%$ pts & 28.6 & 72.7 & $31.3^{*}$ & $11.8^{*}$ & $8.3^{*}$ \\
\hline$\Delta$ COPM-P, points & $2.9(1.7)$ & $4.0(1.6)$ & $3.2(1.3)$ & $2.8(1.6)^{*}$ & $1.5(1.5)^{*+1}$ \\
\hline$\geq 2$ points or more, $\%$ pts & 73.3 & 90.3 & 85.0 & 71.8 & $36.0 *+9$ \\
\hline$\geq 4$ points or more, $\%$ pts & 31.1 & 58.1 & 30.0 & 28.2 & $4.0^{*}$ \\
\hline$\Delta$ COPM-S, points & $3.6(2.0)$ & $4.7(2.0)$ & $4.1(1.6)$ & $3.5(1.7)^{*}$ & $1.7(1.7)^{*+1}$ \\
\hline$\geq 2$ points or more, $\%$ pts & 80.0 & 90.3 & 95.0 & 79.5 & $44.0 *+\emptyset$ \\
\hline$\geq 4$ points or more, $\%$ pts & 44.4 & 77.4 & 47.5 & $38.5^{*}$ & $8.0 *+\emptyset$ \\
\hline$\Delta$ HADS-A, points & $-1.9(3.8)$ & $-3.7(4.1)$ & $-2.4(4.2)$ & $-1.3(2.8)^{*}$ & $0.2(2.6)^{*+}$ \\
\hline$\geq-1.5$ points or more, $\%$ pts & 46.2 & 66.7 & 48.8 & 42.9 & $20.8^{*}$ \\
\hline$\geq-3.0$ points or more, $\%$ pts & 39.2 & 66.7 & 41.5 & $28.6^{*}$ & $16.7^{*}$ \\
\hline$\Delta$ HADS-D, points & $-2.1(3.9)$ & $-4.4(4.0)$ & $-2.8(3.7)$ & $-1.3(2.7)^{*}$ & $-1.0(3.0)^{*+1}$ \\
\hline$\geq-1.5$ points or more, $\%$ pts & 51.5 & 80.0 & 65.9 & $34.3^{*+}$ & $16.7^{*+}$ \\
\hline$\geq-3.0$ points or more, $\%$ pts & 40.8 & 73.3 & 48.8 & $22.9 *$ & $12.5^{*+}$ \\
\hline$\Delta \mathrm{SGRQ}-\mathrm{T}$, points & $-6.6(14.4)$ & $-22.0(12.8)$ & $-8.8(9.7)^{*}$ & $-3.4(6.1)^{*+}$ & $8.0(14.7)^{*+1}$ \\
\hline$\geq-4$ points or more, $\%$ pts & 50.9 & 95.5 & $62.5^{*}$ & $39.4^{*}$ & $4.8 *+9$ \\
\hline$\geq-8$ points or more, $\%$ pts & 41.7 & 90.9 & $50.0 *$ & $24.2^{*}$ & $4.8^{*+}$ \\
\hline $\begin{array}{l}\text { Outcome measures with } \geq 1 x \\
\text { MCID, \% outcomes }\end{array}$ & $56.4(28.2)$ & $88.5(11.6)$ & $67.5(16.6)^{*}$ & $41.4(16.9)^{* \dagger}$ & $24.7(19.6)^{*+1}$ \\
\hline $\begin{array}{l}\text { Outcome measures with } \geq 2 x \\
\text { MCID, \% outcomes }\end{array}$ & $33.6(29.4)$ & $75.6(22.7)$ & $34.3(15.7)^{*}$ & $17.3(13.4)^{*+}$ & $8.1(12.1)^{*+9}$ \\
\hline $\begin{array}{l}\text { Negative outcomes, } \% \\
\text { outcomes }\end{array}$ & $16.7(19.8)$ & $4.4(7.6)$ & $7.0(9.3)$ & $19.7(17.8)^{*+}$ & $41.3(20.6)^{*+9}$ \\
\hline
\end{tabular}

Data are presented as means (SD), unless otherwise stated. $\Delta$, change; MRC, Medical Research Council dyspnea grade; 6MWD, 6-min walk distance; CWRT, constant work-rate test; COPM-P, Canadian Occupational Performance Measure, performance score; COPM-S, Canadian Occupational Performance Measure, satisfaction score; HADS-A, Hospital Anxiety and Depression Scale, anxiety scores; HADS-D, Hospital Anxiety and Depression Scale, depression scores; SGRQ-T, St. George's Respiratory Questionnaire, total Score; MCID, Minimal Clinically Important Difference. *, $p<0.01$ versus cluster 'Very good responder'; $\dagger, p<0.01$ versus cluster 'Good responder'; $\emptyset$, $\mathrm{p}<0.01$ versus cluster 'Moderate responder'. 
Table S5.6 Baseline characteristics of 1,012 male COPD patients without long-term oxygen therapy.

\begin{tabular}{|c|c|c|c|c|}
\hline Baseline & $\begin{array}{l}\text { Very good } \\
\text { responder }\end{array}$ & $\begin{array}{c}\text { Good } \\
\text { responder }\end{array}$ & $\begin{array}{l}\text { Moderate } \\
\text { responder }\end{array}$ & $\begin{array}{c}\text { Poor } \\
\text { responder }\end{array}$ \\
\hline Patients n (\%) & $276(27.3)$ & $299(29.6)$ & $185(18.3)$ & $252(24.9)$ \\
\hline Age, years & $64.6(9.3)$ & $65.5(8.77)$ & $66.5(8.4)$ & $65.7(8.2)$ \\
\hline $\mathrm{FEV}_{1}, \mathrm{I}$ & $1.57(0.64)$ & $1.52(0.55)$ & $1.49(0.59)$ & $1.53(0.63)$ \\
\hline $\mathrm{FEV}_{1}, \%$ predicted & $50.9(19.7)$ & $49.7(16.2)$ & $48.9(17.7)$ & $50.2(19.4)$ \\
\hline KCO, \% predicted & $73.9(24.0)$ & $73.1(23.6)$ & $65.7(21.5)^{*+}$ & $69.6(24.3)$ \\
\hline $\mathrm{PaO}_{2}, \mathrm{kPa}$ & $9.63(1.35)$ & $9.61(1.27)$ & $9.65(1.30)$ & $9.63(1.26)$ \\
\hline $\mathrm{PaCO}_{2}, \mathrm{kPa}$ & $5.15(0.63)$ & $5.19(0.62)$ & $5.18(0.53)$ & $5.13(0.58)$ \\
\hline $\mathrm{SaO}_{2}, \%$ & $94.9(2.3)$ & $94.9(2.2)$ & $95.1(1.9)$ & $95.0(2.1)$ \\
\hline MRC, grade & $3.34(1.12)$ & $2.45(0.93)^{*}$ & $3.04(0.98)^{*}$ & $3.10(1.09)$ \\
\hline Exacerb. $<12 \mathrm{~m}, \mathrm{n}$ & $1.96(2.53)$ & $1.66(2.30)$ & $1.94(2.68)$ & $1.88(2.15)$ \\
\hline Admission $<12 \mathrm{~m}, \mathrm{n}$ & $0.61(1.29)$ & $0.40(0.95)$ & $0.36(0.80)$ & $0.57(1.33)$ \\
\hline CC index, points & $1.47(1.17)$ & $1.60(1.31)$ & $1.57(1.27)$ & $1.48(1.20)$ \\
\hline $\mathrm{BMI}, \mathrm{kg} / \mathrm{m}^{2}$ & $26.5(5.3)$ & $26.2(5.0)$ & $24.6(4.0)^{*+}$ & $25.6(5.1)$ \\
\hline FFMI, $\mathrm{kg} / \mathrm{m}^{2}$ & $18.1(2.4)$ & $17.8(2.2)$ & $17.2(1.8)^{*+}$ & $17.7(2.3)$ \\
\hline $6 \mathrm{MWD}, \mathrm{m}$ & $457(122)$ & $484(104)^{*}$ & $491(108) *$ & 469 (106) \\
\hline $6 \mathrm{MWD}, \%$ predicted & $67.9(16.6)$ & $72.5(13.8)^{*}$ & $72.9(15.2)^{*}$ & $70.3(15.6)$ \\
\hline PWR, watts & $82.8(35.8)$ & $85.9(35.5)$ & $81.1(32.4)$ & $81.2(32.4)$ \\
\hline PWR, \% predicted & $50.1(21.5)$ & $54.0(21.5)$ & $51.1(20.8)$ & $51.8(20.9)$ \\
\hline CWRT, s & $329(194)$ & $379(267)$ & $341(269)$ & $361(287)$ \\
\hline COPM-P, points & $4.08(1.31)$ & $4.53(1.29)^{*}$ & $4.57(1.18)^{*}$ & $4.67(1.22)^{*}$ \\
\hline COPM-S, points & $3.59(1.68)$ & $3.91(1.71)$ & $4.07(1.58)^{*}$ & $4.43(1.72)^{*+}$ \\
\hline HADS-A, points & $7.33(3.87)$ & $5.67(3.83)^{*}$ & $6.02(4.03)^{*}$ & $6.05(4.08)^{*}$ \\
\hline$\geq 8$ points, $\%$ patients & 48.0 & $27.8^{*}$ & $26.1^{*}$ & $31.4 *$ \\
\hline HADS-D, points & $7.20(3.66)$ & $5.72(3.58)^{*}$ & $6.38(3.94)$ & $5.97(3.80)^{*}$ \\
\hline$\geq 8$ points, $\%$ patients & 45.0 & $30.0 *$ & 36.5 & $31.5^{*}$ \\
\hline SGRQ-T, points & $57.6(16.8)$ & $48.4(16.5)^{*}$ & $51.2(16.3)^{*}$ & $50.0(16.4)^{*}$ \\
\hline BODE index, points & $3.3(2.1)$ & $2.9(1.8)$ & $3.1(1.8)$ & $3.1(1.9)$ \\
\hline ADO index, points & $4.4(1.8)$ & $4.2(1.6)$ & $4.4(1.6)$ & $4.3(1.6)$ \\
\hline Inpatient/outpatient, \% patients & $44 / 56$ & $27 / 73^{*}$ & $23 / 77^{*}$ & $18 / 82^{*}$ \\
\hline
\end{tabular}

Data are presented as mean (SD), unless otherwise stated. FEV1, forced expiratory volume in $1 \mathrm{~s}$; KCO, transfer factor of the lung for carbon monoxide; $\mathrm{PaO}_{2}$, arterial oxygen tension; $\mathrm{PaCO}_{2}$, arterial carbon dioxide tension; $\mathrm{SaO}_{2}$, arterial oxygen saturation; MRC, Medical Research Council dyspnea grade; CC index, Charlson Comorbidity Index; BMI, body mass index; FFMI, fat-free Mass Index; 6MWD, 6-min walk distance; PWR, peak work rate; CWRT, constant work-rate test; COPM-P, Canadian Occupational Performance Measure, performance score; COPM-S, Canadian Occupational Performance Measure, satisfaction score; HADS-A, Hospital Anxiety and Depression Scale, anxiety scores; HADS-D Hospital Anxiety and Depression Scale, depression scores; SGRQ-T, St. George's Respiratory Questionnaire, total score; BODE, body mass index, airflow obstruction, dyspnoea, exercise capacity; ADO, age, dyspnoea, airflow obstruction. * ${ }^{*}<0.01$ versus cluster 'Very good responder'; †, p<0.01 versus cluster 'Good responder'. 
Table S5.7 Baseline characteristics of 740 female COPD patients without long-term oxygen.

\begin{tabular}{|c|c|c|c|c|}
\hline Baseline & $\begin{array}{l}\text { Very good } \\
\text { responder }\end{array}$ & $\begin{array}{c}\text { Good } \\
\text { responder }\end{array}$ & $\begin{array}{l}\text { Moderate } \\
\text { responder }\end{array}$ & $\begin{array}{c}\text { Poor } \\
\text { responder }\end{array}$ \\
\hline Patients n (\%) & $169(22.8)$ & $260(35.1)$ & $182(24.6)$ & $129(17.4)$ \\
\hline Age, years & $60(8)$ & $61(9)$ & $62(9)$ & $61(10)$ \\
\hline $\mathrm{FEV}_{1}, \mathrm{I}$ & $1.2(0.5)$ & $1.2(0.4)$ & $1.2(0.4)$ & $1.2(0.5)$ \\
\hline $\mathrm{FEV}_{1}, \%$ predicted & $50.7(18.3)$ & $53.4(17.6)$ & $53.3(17.3)$ & $52.9(18.8)$ \\
\hline $\mathrm{KCO}, \%$ predicted & $64.8(21.4)$ & $63.9(21.3)$ & $60.6(19.9)$ & $61.2(18.9)$ \\
\hline $\mathrm{PaO}_{2}, \mathrm{kPa}$ & $9.6(1.4)$ & $9.6(1.3)$ & $9.8(1.4)$ & $9.7(1.3)$ \\
\hline $\mathrm{PaCO}_{2}, \mathrm{kPa}$ & $5.7(0.7)$ & $5.2(0.6)$ & $5.1(0.6)$ & $5.2(0.6)$ \\
\hline $\mathrm{SaO}_{2}, \%$ & $94.8(2.8)$ & $95.1(2.4)$ & $95.3(2.2)$ & $95.1(2.1)$ \\
\hline MRC, grade & $3.5(1.1)$ & $3.2(1.1)^{*}$ & $3.1(1.1)^{*}$ & $3.1(1.0)^{*}$ \\
\hline Exacerb. $<12 \mathrm{~m}, \mathrm{n}$ & $2.5(2.3)$ & $2.1(2.3)$ & $1.9(2.1)$ & $1.9(1.9)$ \\
\hline Admission $<12 \mathrm{~m}, \mathrm{n}$ & $0.9(1.5)$ & $0.6(1.0)$ & $0.5(1.1)$ & $0.4(0.8)$ \\
\hline CC index, points & $1.1(1.0)$ & $1.2(1.0)$ & $1.2(0.8)$ & $1.1(0.9)$ \\
\hline $\mathrm{BMI}, \mathrm{kg} / \mathrm{m}^{2}$ & $25.4(5.5)$ & $25.3(6.1)$ & $24.7(5.3)$ & $24.4(4.7)$ \\
\hline FFMI, $\mathrm{kg} / \mathrm{m}^{2}$ & $15.5(1.9)$ & $15.3(2.0)$ & $15.3(1.9)$ & $15.2(1.7)$ \\
\hline 6MWD, m & 433 (119) & 443 (99) & $464(91)^{*}$ & $474(93)^{*+}$ \\
\hline 6MWD, \% predicted & $69.9(18.1)$ & $73.9(14.5)$ & $77.4(13.5)^{*}$ & $77.7(13.8)^{*}$ \\
\hline PWR, watts & $63.7(26.5)$ & $64.8(22.8)$ & $64.1(21.8)$ & $66.6(22.2)$ \\
\hline PWR, \% predicted & $63.0(27.8)$ & $70.4(30.2)$ & $70.0(24.7)$ & $69.1(27.5)$ \\
\hline CWRT, s & $291(168)$ & $294(225)$ & $298(231)$ & $315(254)$ \\
\hline COPM-P, points & $4.1(1.2)$ & $4.1(1.3)$ & $4.3(1.3)$ & $4.6(1.4)^{*+}$ \\
\hline COPM-S, points & $3.2(1.5)$ & $3.4(1.7)$ & $3.7(1.7)$ & $3.9(1.9)^{*}$ \\
\hline HADS-A, points & $8.8(4.2)$ & $8.1(4.4)$ & $7.7(4.3)$ & $7.1(4.2)^{*}$ \\
\hline$\geq 8$ points, $\%$ patients & 58.5 & 53.8 & 47.7 & 46.7 \\
\hline HADS-D, points & $7.5(4.4)$ & $7.1(4.1)$ & $6.7(4.1)$ & $6.0(4.0)^{*}$ \\
\hline$\geq 8$ points, $\%$ patients & 46.3 & 44.9 & 39.8 & 33.9 \\
\hline SGRQ-T, points & $57.5(15.9)$ & $52.2(15.9)^{*}$ & $50.2(16.8)^{*}$ & $46.8(18.3)^{*+}$ \\
\hline BODE index, points & $3.7(2.2)$ & $3.0(2.0)^{*}$ & $2.9(1.9)^{*}$ & $2.9(1.8)^{*}$ \\
\hline ADO index, points & $4.0(1.7)$ & $3.8(1.8)$ & $3.9(1.5)$ & $3.7(1.6)$ \\
\hline Inpatients/outpatients, \% patients & $53 / 47$ & $38 / 62^{*}$ & $28 / 72^{*}$ & $20 / 80 *+$ \\
\hline
\end{tabular}

Data are presented as mean (SD), unless otherwise stated. $\mathrm{FEV}_{1}$, forced expiratory volume in $1 \mathrm{~s}$; KCO, transfer factor of the lung for carbon monoxide; $\mathrm{PaO}_{2}$, arterial oxygen tension; $\mathrm{PaCO}_{2}$, arterial carbon dioxide tension; $\mathrm{SaO}_{2}$, arterial oxygen saturation; MRC, Medical Research Council dyspnea grade; CC index, Charlson Comorbidity Index; BMI, body mass index; FFMI, fat-free Mass Index; 6MWD, 6-min walk distance; PWR, peak work rate; CWRT, constant work-rate test; COPM-P, Canadian Occupational Performance Measure, performance score; COPM-S, Canadian Occupational Performance Measure, satisfaction score; HADS-A, Hospital Anxiety and Depression Scale, anxiety scores; HADS-D Hospital Anxiety and Depression Scale, depression scores; SGRQ-T, St. George's Respiratory Questionnaire, total score; BODE, body mass index, airflow obstruction, dyspnoea, exercise capacity; ADO, age, dyspnoea, airflow obstruction. *, p<0.01 versus cluster 'Very good responder'; †, p<0.01 versus cluster 'Good responder'. 
Table S5.8 Baseline characteristics of 168 male COPD patients with long-term oxygen therapy.

\begin{tabular}{|c|c|c|c|c|}
\hline Baseline & $\begin{array}{l}\text { Very good } \\
\text { responder }\end{array}$ & $\begin{array}{c}\text { Good } \\
\text { responder }\end{array}$ & $\begin{array}{l}\text { Moderate } \\
\text { responder }\end{array}$ & $\begin{array}{c}\text { Poor } \\
\text { responder }\end{array}$ \\
\hline Patients n (\%) & $52(31.0)$ & $58(34.5)$ & $34(20.2)$ & $24(14.3)$ \\
\hline Age, years & $65(8)$ & $67(8)$ & $66(7)$ & $68(7)$ \\
\hline $\mathrm{FEV}_{1}, \mathrm{I}$ & $1.1(0.5)$ & $1.0(0.3)$ & $1.0(0.4)$ & $0.8(0.2)$ \\
\hline $\mathrm{FEV}_{1}, \%$ predicted & $35.6(16)$ & $32.9(12.1)$ & $32.0(12.0)$ & $28.1(8.6)$ \\
\hline $\mathrm{KCO}, \%$ predicted & $58.8(21.3)$ & $61.7(24.5)$ & $62.3(21.0)$ & $62.3(25.1)$ \\
\hline $\mathrm{PaO}_{2}, \mathrm{kPa}$ & 9.7 (1.9) & $10.0(1.7)$ & $9.5(1.5)$ & $9.5(1.3)$ \\
\hline $\mathrm{PaCO}_{2}, \mathrm{kPa}$ & $6.0(1.4)$ & $5.8(1.0)$ & $5.9(1.1)$ & $6.4(1.3)$ \\
\hline $\mathrm{SaO}_{2}, \%$ & $94.7(2.4)$ & $95.1(2.6)$ & $94.7(2.1)$ & $94.6(2.4)$ \\
\hline MRC, grade & $4.3(1.1)$ & $4.1(1.0)$ & $4.0(1.0)$ & $4.2(1.0)$ \\
\hline Exacerb. $<12 \mathrm{~m}, \mathrm{n}$ & $3.1(2.2)$ & $3.2(3.3)$ & $2.5(2.6)$ & $3.4(1.8)$ \\
\hline Admission $<12 \mathrm{~m}, \mathrm{n}$ & $2.5(2.6)$ & $2.2(2.2)$ & $1.6(2.0)$ & $1.8(1.8)$ \\
\hline CC index, points & $1.7(1.4)$ & $1.7(1.6)$ & $1.5(1.4)$ & $1.5(0.8)$ \\
\hline $\mathrm{BMI}, \mathrm{kg} / \mathrm{m}^{2}$ & $27.3(6.2)$ & $26.5(5.3)$ & $26.5(4.7)$ & $22.9(3.4)^{*+9}$ \\
\hline FFMI, $\mathrm{kg} / \mathrm{m}^{2}$ & $18.5(2.8)$ & $17.6(2.0)$ & $18.0(2.2)$ & $16.0(1.9)^{*+1}$ \\
\hline $6 \mathrm{MWD}, \mathrm{m}$ & $334(92)$ & $362(113)$ & $368(124)$ & $381(95)$ \\
\hline $6 \mathrm{MWD}, \%$ pred & $50.7(14.8)$ & $55.9(16.3)$ & $56.7(18.5)$ & $59.3(11.6)$ \\
\hline PWR, watts & $57.4(19.7)$ & $56.3(16.6)$ & $58.6(19.8)$ & $53.5(15.5)$ \\
\hline PWR, \% pred & $35.7(14.2)$ & $38.0(15.5)$ & 36.9 (11.9) & $36.8(14.1)$ \\
\hline CWRT, s & $237(115)$ & 245 (198) & $231(110)$ & $237(232)$ \\
\hline COPM-P, points & $3.5(1.5)$ & $3.7(1.5)$ & $4.0(1.4)$ & $3.6(1.0)$ \\
\hline COPM-S, points & $3.4(1.5)$ & $3.3(1.5)$ & $3.8(1.7)$ & $3.5(1.8)$ \\
\hline HADS-A, points & $8.5(4.9)$ & $8.1(4.8)$ & $6.7(4.5)$ & $6.3(4.4)$ \\
\hline$\geq 8$ points, $\%$ patients & 57.4 & 46.4 & 31.3 & 40.9 \\
\hline HADS-D, points & $8.6(4.1)$ & $8.0(4.5)$ & $6.9(4.1)$ & $7.3(4.5)$ \\
\hline$\geq 8$ points, $\%$ patients & 61.7 & 50.0 & 50.0 & 54.5 \\
\hline SGRQ-T, points & $69.2(12.3)$ & $64.5(15.6)$ & $57.8(16.5)^{*}$ & $60.0(12.5)^{*}$ \\
\hline BODE index, points & $5.6(1.9)$ & $5.4(1.9)$ & $5.2(1.9)$ & $5.7(1.6)$ \\
\hline ADO index, points & $5.8(1.2)$ & $6.0(1.6)$ & $5.9(1.2)$ & $6.4(1.3)$ \\
\hline Inpatients/outpatients, \% patients & $96 / 4$ & $88 / 12$ & $65 / 35^{*+}$ & $83 / 17$ \\
\hline
\end{tabular}

Data are presented as mean (SD), unless otherwise stated. $\mathrm{FEV}_{1}$, forced expiratory volume in $1 \mathrm{~s}$; $\mathrm{KCO}$, transfer factor of the lung for carbon monoxide; $\mathrm{PaO}_{2}$, arterial oxygen tension; $\mathrm{PaCO}_{2}$, arterial carbon dioxide tension; $\mathrm{SaO}_{2}$, arterial oxygen saturation; MRC, Medical Research Council dyspnea grade; CC index, Charlson Comorbidity Index; BMI, body mass index; FFMI, fat-free Mass Index; 6MWD, 6-min walk distance; PWR, peak work rate; CWRT, constant work-rate test; COPM-P, Canadian Occupational Performance Measure, performance score; COPM-S, Canadian Occupational Performance Measure, satisfaction score; HADS-A, Hospital Anxiety and Depression Scale, anxiety scores; HADS-D Hospital Anxiety and Depression Scale, depression scores; SGRQ-T, St. George's Respiratory Questionnaire, total score; BODE, body mass index, airflow obstruction, dyspnoea, exercise capacity; ADO, age, dyspnoea, airflow obstruction. *, p<0.01 versus cluster 'Very good responder'; †, $p<0.01$ versus cluster 'Good responder'; $\uparrow$, $p<0.01$ versus 'Moderate responder'. 
Table S5.9 Baseline characteristics of 148 female COPD patients with long-term oxygen therapy.

\begin{tabular}{|c|c|c|c|c|}
\hline Baseline & $\begin{array}{l}\text { Very good } \\
\text { responder }\end{array}$ & $\begin{array}{c}\text { Good } \\
\text { responder }\end{array}$ & $\begin{array}{l}\text { Moderate } \\
\text { responder }\end{array}$ & $\begin{array}{c}\text { Poor } \\
\text { responder }\end{array}$ \\
\hline Patients n (\%) & $33(22.3)$ & $44(29.7)$ & $42(28.4)$ & $29(19.6)$ \\
\hline Age, years & $63(7)$ & $65(8)$ & $65(7)$ & $65(8)$ \\
\hline $\mathrm{FEV}_{1}, \mathrm{I}$ & $0.8(0.4)$ & $0.8(0.3)$ & $0.7(0.2)$ & $0.7(0.2)$ \\
\hline $\mathrm{FEV}_{1}, \%$ predicted & $36(18)$ & $36(13)$ & $34(12)$ & $36(12)$ \\
\hline KCO, \% predicted & $53(15)$ & $54(20)$ & $50(17)$ & $53(17)$ \\
\hline $\mathrm{PaO}_{2}, \mathrm{kPa}$ & $9.0(1.2)$ & $9.5(1.3)$ & $9.5(1.4)$ & $9.6(2.3)$ \\
\hline $\mathrm{PaCO}_{2}, \mathrm{kPa}$ & $6.6(1.4)$ & $6.2(1.0)$ & $6.4(1.0)$ & $6.2(0.8)$ \\
\hline $\mathrm{SaO}_{2}, \%$ & $93.9(2.9)$ & $94.5(2.7)$ & $94.7(2.7)$ & $94.4(3.0)$ \\
\hline MRC, grade & $4.7(0.6)$ & $3.8(1.1)^{*}$ & $4.2(1.1)$ & $4.0(1.1)^{*}$ \\
\hline Exacerb. $<12 \mathrm{~m}, \mathrm{n}$ & $2.9(1.7)$ & $3.5(3.6)$ & $2.9(2.6)$ & $2.5(2.2)$ \\
\hline Admission $<12 \mathrm{~m}, \mathrm{n}$ & $2.0(1.6)$ & $1.5(1.5)$ & $1.6(1.5)$ & $1.3(1.5)$ \\
\hline CC index, points & $1.2(0.9)$ & $1.1(0.9)$ & $1.6(1.2)$ & $1.4(0.9)$ \\
\hline $\mathrm{BMI}, \mathrm{kg} / \mathrm{m}^{2}$ & $26.3(6.9)$ & $25.3(5.9)$ & $25.7(4.8)$ & $27.1(6.7)$ \\
\hline FFMI, $\mathrm{kg} / \mathrm{m}^{2}$ & $15.8(2.6)$ & $15.5(2.3)$ & $15.5(1.7)$ & $16.3(2.9)$ \\
\hline 6MWD, m & $279(92)$ & $366(104)^{*}$ & $353(97)^{*}$ & $353(109)^{*}$ \\
\hline $6 \mathrm{MWD}, \%$ pred & $50.0(15.5)$ & $67.2(13.9)^{*}$ & $64.4(14.9)^{*}$ & $61.8(18.8)$ \\
\hline PWR, watts & $41(17)$ & $49(16)$ & $47(14)$ & $46(14)$ \\
\hline PWR, \% pred & $50.2(22.4)$ & $59.1(17.9)$ & $55.4(22.8)$ & $56.7(24.8)$ \\
\hline CWRT, s & 202 (130) & 239 (95) & $178(71)+$ & $233(140)$ \\
\hline COPM-P, points & $3.1(1.4)$ & $3.8(1.3)$ & $3.6(1.3)$ & $4.3(1.6)^{*}$ \\
\hline COPM-S, points & $2.6(1.5)$ & $3.1(1.5)$ & $3.0(1.4)$ & $4.2(1.4)^{*+1}$ \\
\hline HADS-A, points & $9.8(4.5)$ & $9.0(4.4)$ & $8.5(5.3)$ & $8.3(4.6)$ \\
\hline$\geq 8$ points, $\%$ patients & 68.0 & 67.0 & 54.0 & 67.0 \\
\hline HADS-D, points & $9.0(4.8)$ & $8.0(4.4)$ & $7.7(4.1)$ & $6.9(4.9)$ \\
\hline$\geq 8$ points, $\%$ patients & 68.7 & 55.0 & 49.0 & 46.0 \\
\hline SGRQ-T, points & $68.7(10.9)$ & $62.9(14.9)$ & $61.3(12.5)$ & $56.1(14.5)^{*}$ \\
\hline BODE index, points & $6.4(2.2)$ & $4.9(2.1)^{*}$ & $5.6(1.9)$ & $5.3(2.1)$ \\
\hline ADO index, points & $6.1(1.2)$ & $5.3(1.6)$ & $5.9(1.2)$ & $5.5(1.3)$ \\
\hline Inpatients/outpatients, \% patients & $91 / 9$ & $88 / 12$ & $85 / 15$ & $89 / 11$ \\
\hline
\end{tabular}

Data are presented as mean (SD), unless otherwise stated. $\mathrm{FEV}_{1}$, forced expiratory volume in $1 \mathrm{~s}$; KCO, transfer factor of the lung for carbon monoxide; $\mathrm{PaO}_{2}$, arterial oxygen tension; $\mathrm{PaCO}_{2}$, arterial carbon dioxide tension; $\mathrm{SaO}_{2}$, arterial oxygen saturation; MRC, Medical Research Council dyspnea grade; CC index, Charlson Comorbidity Index; BMI, body mass index; FFMI, fat-free Mass Index; 6MWD, 6-min walk distance; PWR, peak work rate; CWRT, constant work-rate test; COPM-P, Canadian Occupational Performance Measure, performance score; COPM-S, Canadian Occupational Performance Measure, satisfaction score; HADS-A, Hospital Anxiety and Depression Scale, anxiety scores; HADS-D Hospital Anxiety and Depression Scale, depression scores; SGRQ-T, St. George's Respiratory Questionnaire, total score; BODE, body mass index, airflow obstruction, dyspnoea, exercise capacity; ADO, age, dyspnoea, airflow obstruction. *, p<0.01 versus cluster 'Very good responder'; †, $p<0.01$ versus cluster 'Good responder'; १, $p<0.01$ versus 'Moderate responder'. 


\section{Legend Figure S5.1 to S5.4}

Panels on next pages were generated using Viscovery software. The Viscovery program placed all patients on a specific position on the map based on their multidimensional response profile. The more subjects resemble in terms of their response to pulmonary rehabilitation the closer they are on the map. Contrarily, the more they differ the further they are away from each other. When looking at an outcome measure of pulmonary rehabilitation, patients "raise a red flag" if they had a very good response, "a green flag" when the response was good to moderate, and "a blue flag" when the response was absent. In this way the maps can be interpreted. Using the topology of the, the Viscovery program could identify four different clusters of patients with COPD with a significantly different multidimensional response profile: C1: cluster 1 'Very good responder'; C2: cluster 2 'Good responder'; C3: cluster 3 'Moderate responder'; C4: cluster 4 'Poor responder'. Figure S5.1: 1,012 male COPD patients without long-term oxygen therapy; Figure S5.2: 740 female COPD patients without long-term oxygen therapy; Figure S5.3: 168 male COPD patients with long-term oxygen therapy; and Figure S5.4: 148 female COPD patients with long-term oxygen therapy. 

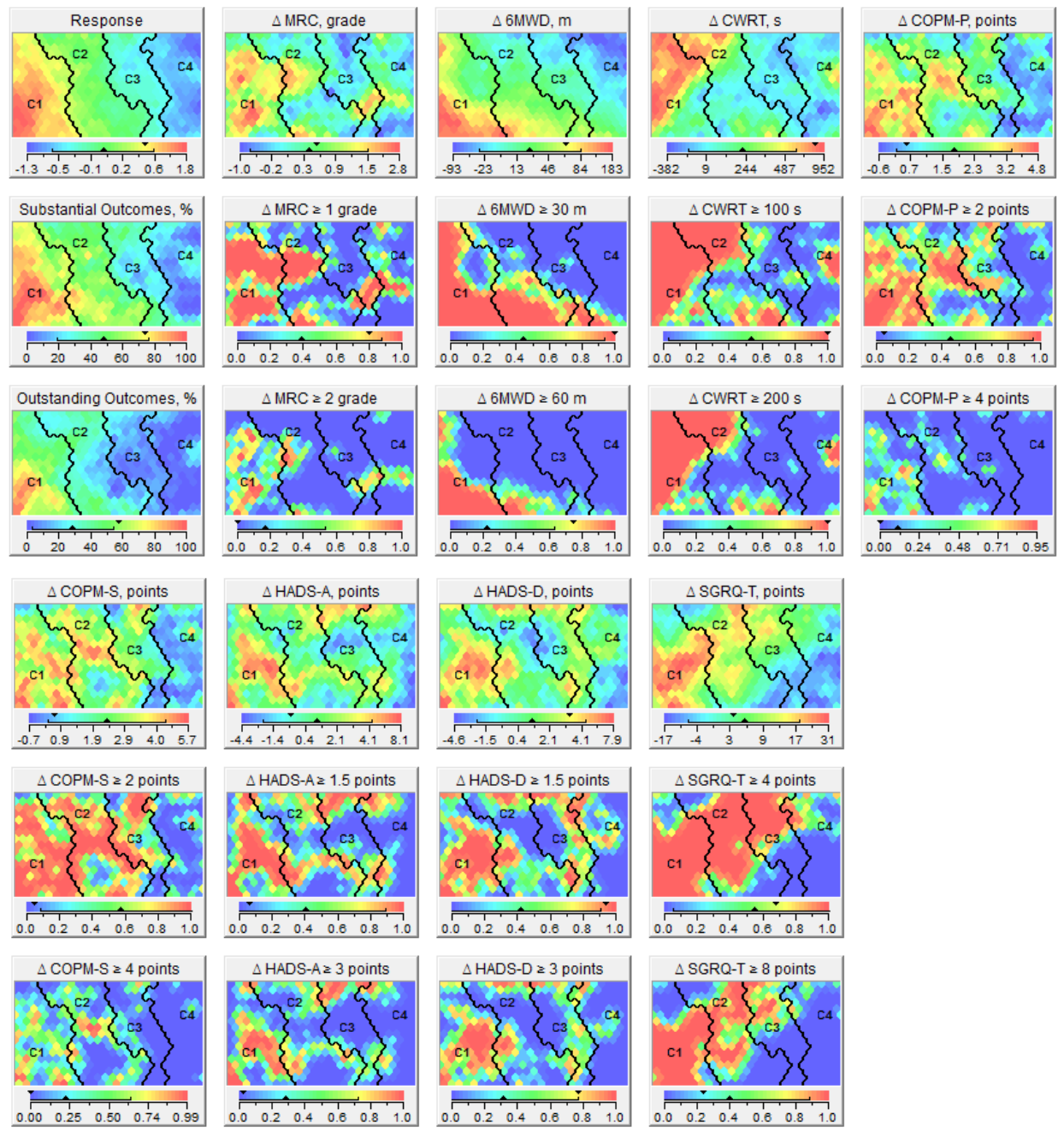

Figure S5.1 Multidimensional response clusters in 1,012 male COPD patients without long-term oxygen therapy. 


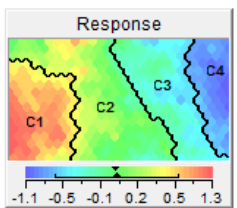

Substantial Outcomes, \%

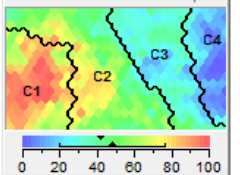

Outstanding Outcomes, \%

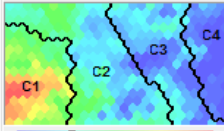

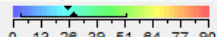

$\triangle$ COPM-S, points

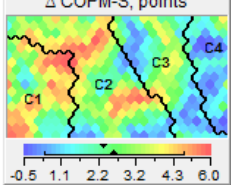

$\triangle$ COPM-S $\geq 2$ points

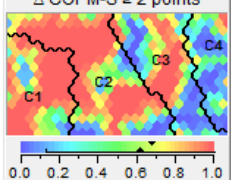

$\triangle$ COPM-S $\geq 4$ points

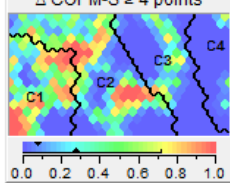

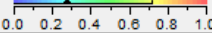
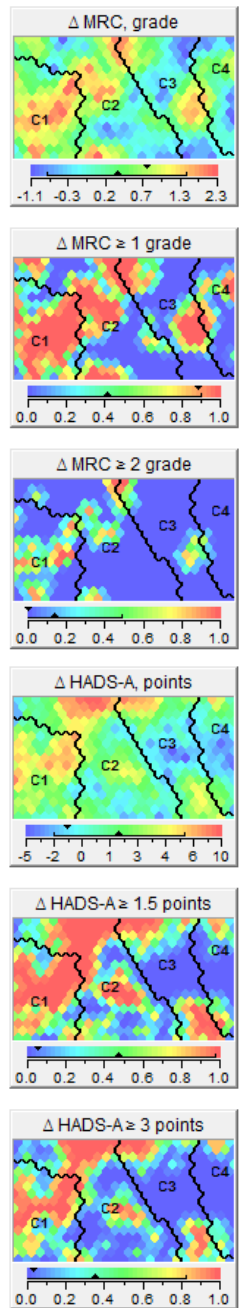
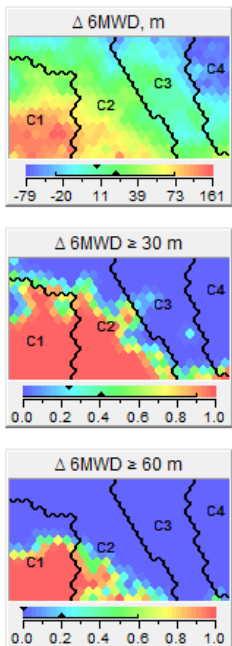

\section{$\triangle$ HADS-D, points}
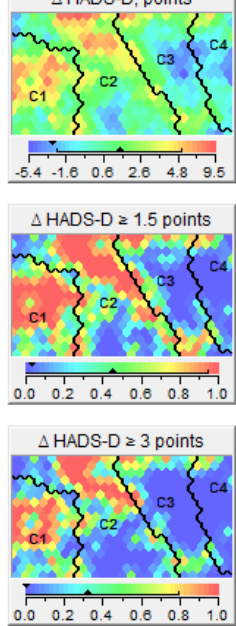
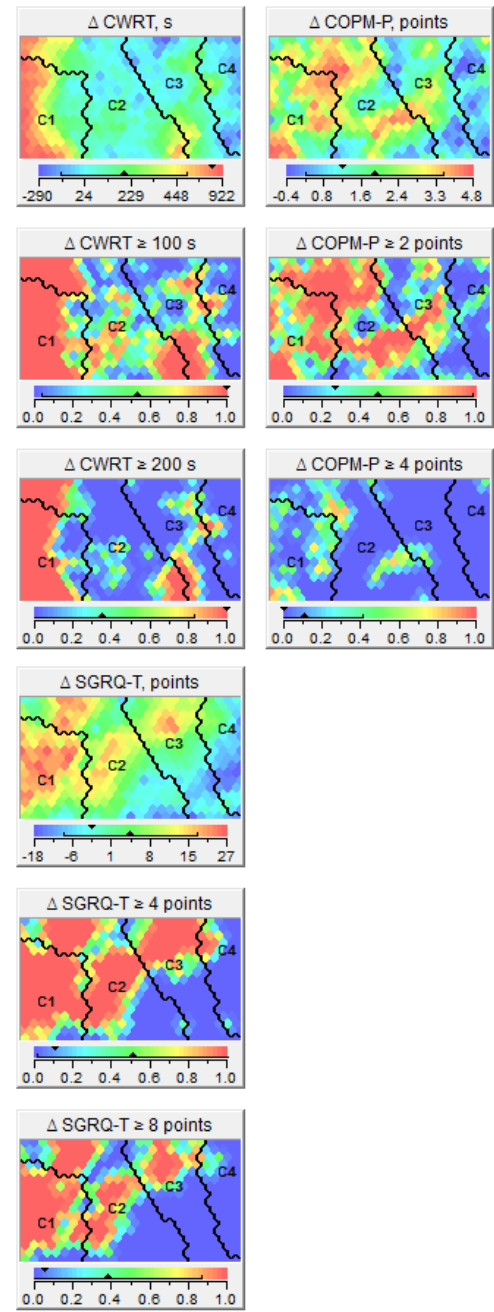

Figure S5.2 Multidimensional response clusters in 740 female COPD patients without long-term oxygen. 


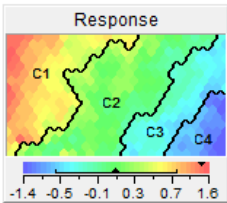

Substantial Outcomes, \%
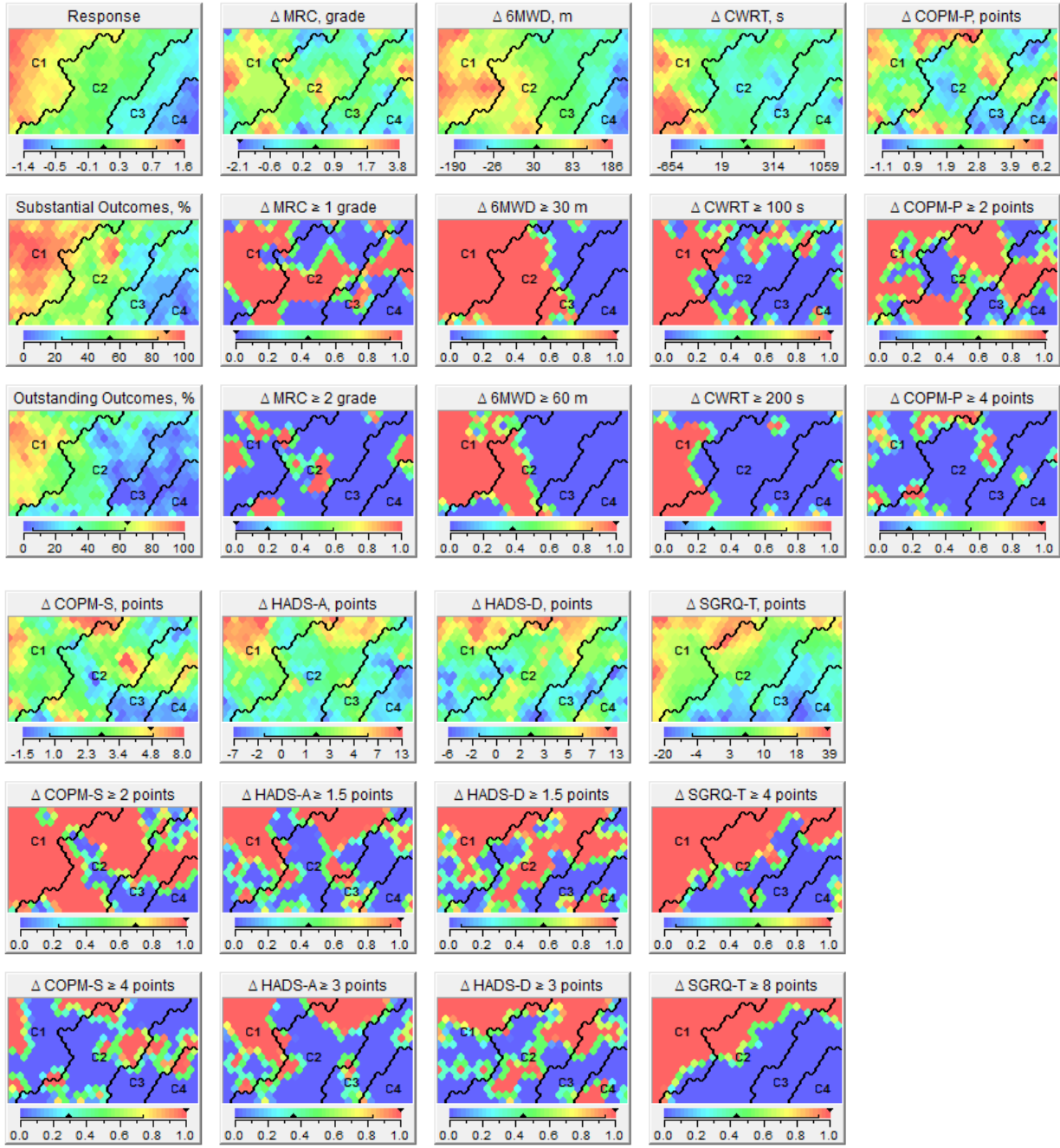
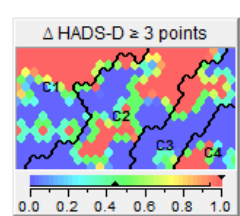

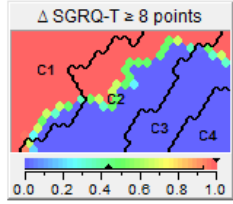

Figure S5.3 Multidimensional response clusters in 168 male COPD patients with long-term oxygen therapy. 

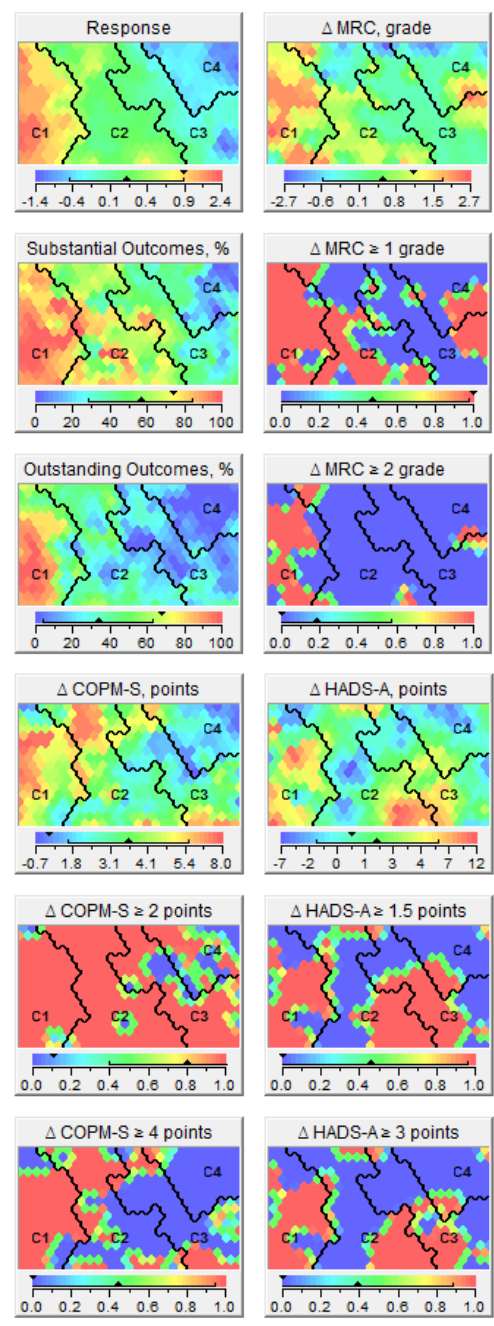
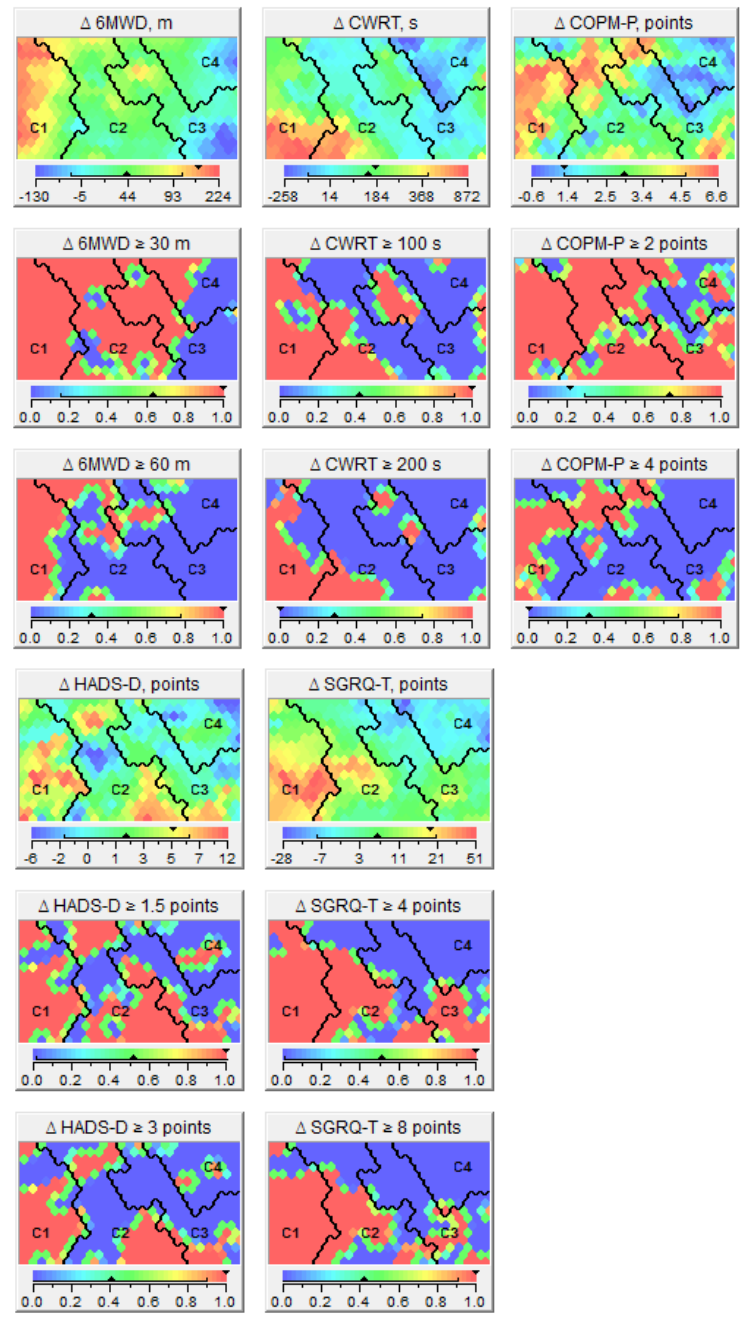

Figure S5.4 Multidimensional response clusters in 148 female COPD patients with long-term oxygen therapy. 




\section{Chapter 6}

\section{Comprehensive lung function assessment does not allow to infer response to pulmonary rehabilitation in patients with COPD}

Ingrid ML Augustin, Emiel FM Wouters, Sarah Houben-Wilke, Swetlana Gaffron, Daisy JA Janssen, Frits ME Franssen, Martijn A Spruit Journal of Clinical Medicine 2018;8(1). 


\section{Abstract}

The degree of lung function is frequently used as referral criterion for pulmonary rehabilitation. The efficacy of pulmonary rehabilitation was assessed in 518 chronic obstructive pulmonary disease (COPD) patients, after clustering based on a comprehensive pre-rehabilitation lung function assessment. Mean improvements in dyspnea, exercise performance, health status, mood status and problematic activities of daily life after pulmonary rehabilitation were mostly comparable between the seven clusters, despite significant differences in the degree of lung function. The current study demonstrates no significant relationship between the seven lung-function-based clusters and response to pulmonary rehabilitation. Therefore, baseline lung function cannot be used to identify those who will respond well to pulmonary rehabilitation, and moreover, cannot be used as a criterion for referral to pulmonary rehabilitation in patients with COPD. 


\section{Introduction}

Pulmonary rehabilitation, defined as a comprehensive non-pharmacological intervention, is generally very effective in patients with chronic obstructive pulmonary disease (COPD). ${ }^{1}$ Indeed, statistically significant and clinically relevant improvements can be obtained for dyspnea, exercise capacity and health status compared to standard care. $^{2-4}$

In daily practice and in clinical trials, the degree of airflow limitation is frequently used as an indicator for referral for pulmonary rehabilitation. ${ }^{2}$ However, not all patients with COPD with severe to very severe airflow limitation are symptomatic or limited in their daily functioning. ${ }^{5}$ Conversely, a proportion of COPD patients with mild to moderate airflow limitation may suffer from severe dyspnea and experience everyday limitations. ${ }^{3}$ The degree of airflow limitation, therefore, is a poor determinant of the physical and psychological status of a patient with COPD., It has been shown that mean improvements following exercise-based pulmonary rehabilitation are comparable after stratification for baseline airflow limitation., ${ }^{8,9}$ Moreover, there is no difference in baseline forced expiratory volume in $1 \mathrm{~s}\left(\mathrm{FEV}_{1}\right)$ between very good and poor responders to pulmonary rehabilitation. ${ }^{3}$ Thus, the degree of airflow limitation is a poor selection criterion for pulmonary rehabilitation. The same is true for the degree of static lung hyperinflation. ${ }^{10}$

Recently, the heterogeneity of respiratory impairment in patients with COPD has been illustrated by the respiratory physiome, in which patients are clustered on multiple lung function attributes. ${ }^{11}$ Whether and to what extent the respiratory physiome can be used as an indicator for referral for pulmonary rehabilitation remains currently unknown. $A$ priori, we hypothesize that the respiratory physiome clusters are unable to infer response to pulmonary rehabilitation in patients with COPD.

\section{Methods}

\section{Study design}

This is an observational, prospective, single-center study about COPD, health status and cardiovascular comorbidities in relation to the outcomes of pulmonary rehabilitation (the CHANCE study). ${ }^{12}$ This study was approved by the Medical Ethical Committee of the Maastricht University Medical Centre+ (METC 11-3-070) and is registered as "Clinical, physiological and psychosocial determinants of the COPD Assessment Test (CAT)", NTR 3416. ${ }^{13}$ 


\section{Study sample}

Patients with COPD referred by chest physicians for a comprehensive pulmonary rehabilitation program at Ciro (Horn, the Netherlands) were included. Ciro is a third line rehabilitation center in Southern Netherlands. It specializes in offering individualized and multidimensional rehabilitation programs to patients with complex respiratory diseases. Only patients with COPD were included, and all patients gave written informed consent.

\section{Measurements}

In total, 518 COPD patients (44\% women; mean $\mathrm{FEV}_{1} 48.6$ [20\% predicted]; 72\% stratified into group $D$ of the Global initiative for Chronic Obstructive Lung Disease [GOLD D]; mean body mass index [BMI] 26.2 [5.8 kg/m²]) were included. Before and after a 40-session comprehensive multidimensional pulmonary rehabilitation program, patients underwent an assessment of lung function and health status characteristics ${ }^{11,12}$ (Figure 6.1). Analysis of the respiratory physiome was based on the pre-rehabilitation comprehensive lung function testing. It included post-bronchodilator spirometry to assess forced expiratory volume in $1 \mathrm{~s}\left(\mathrm{FEV}_{1}\right)$ and forced vital capacity (FVC); bodyplethysmography to determine total lung capacity (TLC), residual volume (RV) and intra thoracic gas volume (ITGV); single-breath determination of carbon monoxide (TLCO); maximal static inspiratory (MIP) and expiratory mouth pressures (MEP); resting arterial partial pressure of oxygen $\left(\mathrm{PaO}_{2}\right)$, carbon dioxide $\left(\mathrm{PaCO}_{2}\right)$ and oxygen saturation $\left(\mathrm{SO}_{2}\right)$. Seven different clusters of lung function impairment could be identified as described in a previous paper ${ }^{11}$ (Figure 6.2). In brief, Cluster 1 had a significantly lower degree of airflow limitation, absence of static hyperinflation, and a higher diffusing capacity compared to the other clusters. Clusters 2 to 4 had similar degree of airflow limitation, but showed significant differences in static lung volumes (Cluster $3>$ Cluster $4>$ Cluster 2 , all $p<0.01$ ). Cluster 5 had a significantly lower degree of airflow limitation compared to Clusters 6 and $7(p<0.01)$. Static lung volumes were significantly different between Clusters 5 to 7 (Cluster $7>$ Cluster $6>$ Cluster 5 , all $p<0.01$ ). Diffusing capacity of the lung for carbon monoxide (DLCO) was higher in Clusters 1, 4 and 5; lower in Clusters 3, 6, and 7, $p<0.01$ and mouth pressures were higher in Clusters 1, 3, 4, and 6; lower in Clusters 2, 5 , and $7, p<0.01$. Arterial blood gas values were within normal range in Clusters $1-6 .{ }^{11}$ The efficacy of pulmonary rehabilitation ${ }^{3}$ was measured by the degree of dyspnea. Dyspnea was measured using the modified Medical Research Council (mMRC) scale, ranging from grade 0 (no troubles with breathlessness) to grade 4 (too breathless to leave the house). The COPD-specific version of the St George's Respiratory Questionnaire (SGRQ-C) was also used, ranging from 0 (optimal) to 100 points (worst). A 6-min walk test (6MWT) was used to assess exercise performance. In addition, a submaximal exercise test (CWRT) was performed at 75\% of the pre-determined peak work rate using an electrically braked cycle ergometer (Carefusion, Houten, the Netherlands). The Canadian Occupational Performance Measure (COPM) was used to identify specific problematic activities of daily life. Patients scored how well they were 
performing the problematic activities of daily life (performance score; COPM-P) and how satisfied they were with this level of performance (satisfaction score; COPM-S). Scores range between 1 ("not able to do it" or "not at all satisfied", respectively) to 10 points ("able to do it extremely well" or "extremely satisfied"). Symptoms of anxiety and depression were measured by the Hospital Anxiety and Depression Scale (HADS) with a total score ranging from 0 (optimal) to 21 (worst) points. A score of 11 or higher indicates a severe mood disturbance.

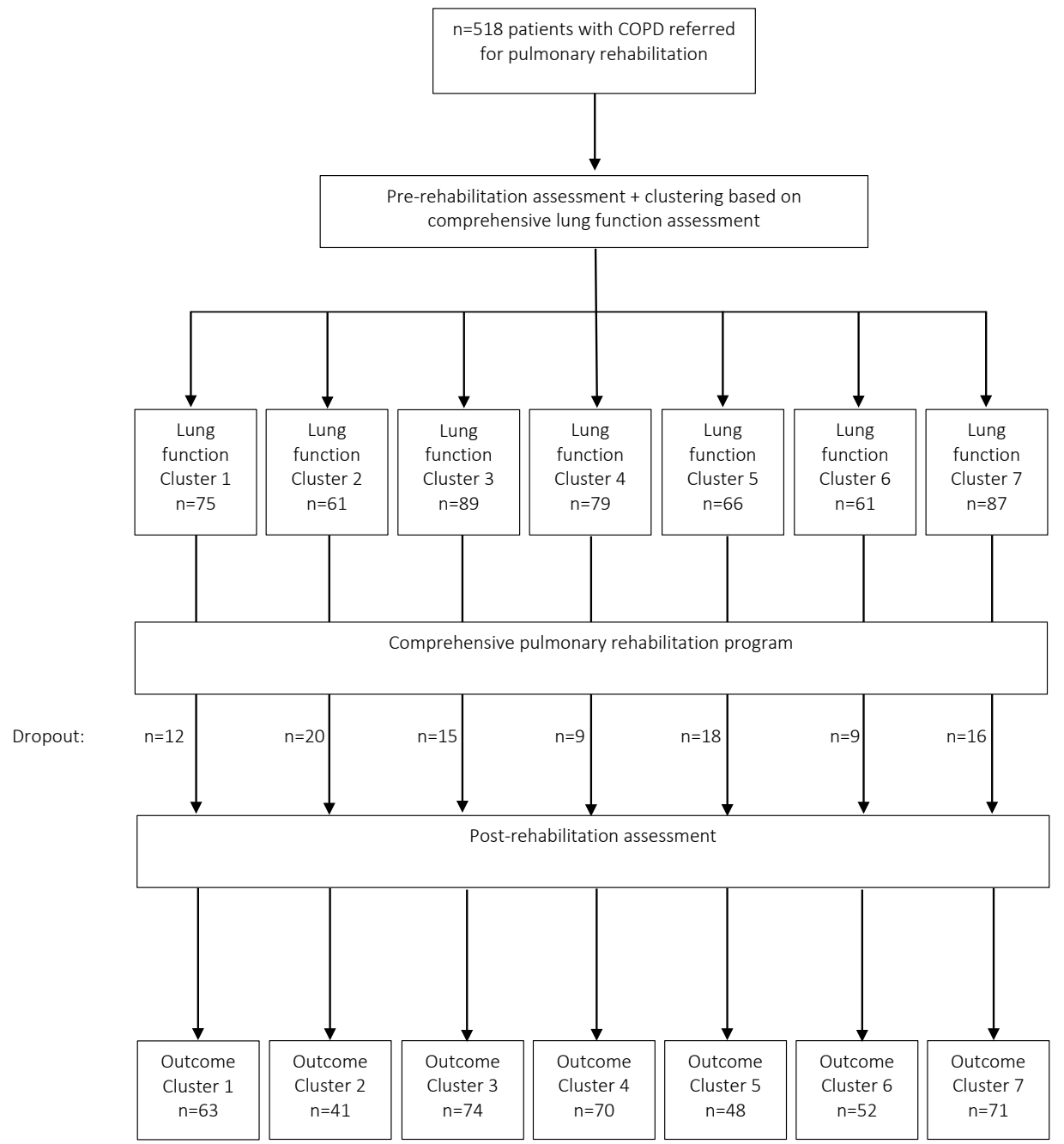

Figure 6.1 Patients before and after a 40-session comprehensive pulmonary rehabilitation program. 


\section{SOM-Ward-Clusters}

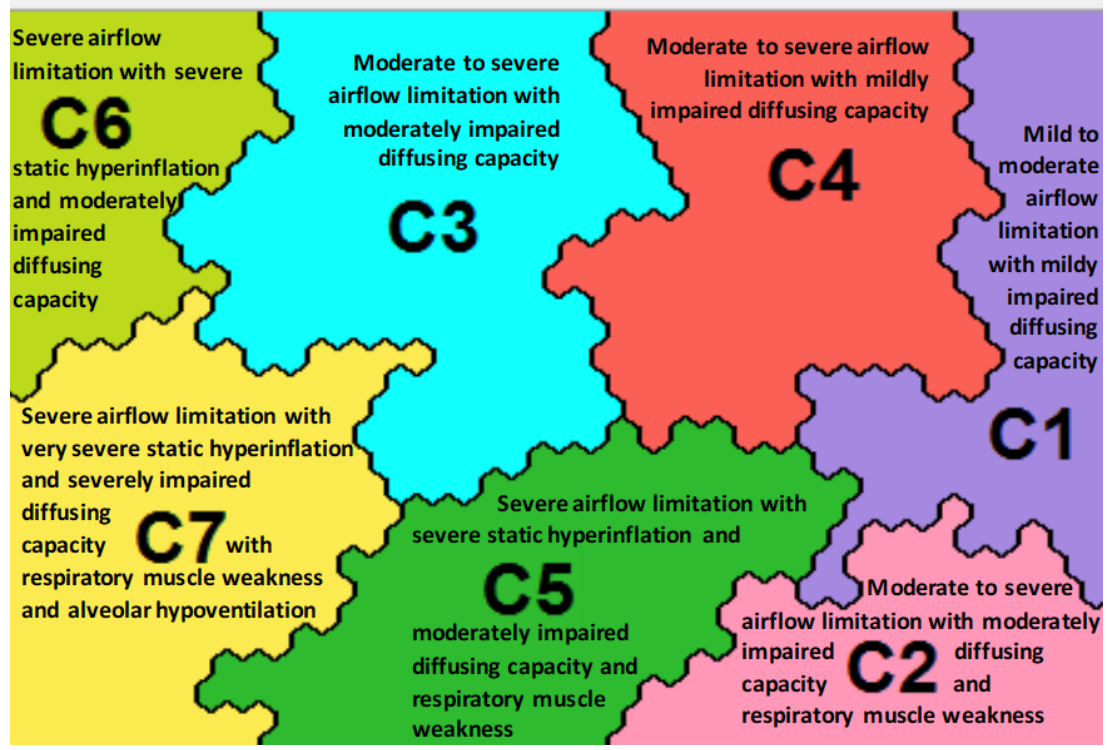

Figure 6.2 Seven different clusters of patients with COPD based on differing respiratory physiome. This figure was published in Augustin et al. ${ }^{11}$ The seven lung function clusters in chronic obstructive pulmonary disease (COPD) using Viscovery (Viscovery Software GmbH, Vienna, Austria). Viscovery program placed all subjects on a specific position on the map based on their profile of a comprehensive lung function assessment. Subjects with similar lung function are closer together on the map and vice versa. By drawing lines on the map, the Viscovery program could identify seven different clusters of patients with COPD with a significantly different respiratory physiome (95\% confidence interval).

\section{Regular intervention}

The pulmonary rehabilitation program was provided in accordance with the 2013 American Thoracic Society/European Respiratory Society Statement on pulmonary rehabilitation $^{1}$, meeting the individual needs of patients with COPD. ${ }^{14}$ The program consists of 40 sessions and can be inpatient ( 8 weeks, 5 days.week-1) or outpatient ( 8 weeks, 3 half days.week-1, followed by 8 weeks 2 half days-week-1). The program starts with a careful characterization of pulmonary and extra-pulmonary treatable traits in patients with COPD. From this, a patient-tailored program consisting of different treatment modules is composed. Each module consists of different interventions; physical exercise training, occupational therapy, nutritional counselling, psychosocial counselling, education and exacerbation management. Each module has a specific goal, which once achieved, contributes to the patients' overall goal(s) of the treatment. ${ }^{14}$ 


\section{Statistics}

All statistical analyses were performed using Viscovery Profiler 7.1 by Viscovery Software $\mathrm{GmbH}$, Vienna, Austria. Information available online. ${ }^{15}$ Self-organizing maps (SOMs, also referred to as Kohonen maps) were used to create an ordered representation of the selected attributes. The SOM method can be viewed as a non-parametric regression technique that converts multidimensional data spaces into lower dimensional abstractions. A SOM generates a non-linear representation of the data distribution and allows the user to identify homogeneous data groups visually. Patients have been ordered by their overall similarity concerning the lung function variables measured during pre-rehabilitation assessment. ${ }^{11}$ Using the SOM-Ward Cluster algorithm of Viscovery, a hybrid algorithm that applies the classical hierarchical method of Ward on top of the SOM topology, the seven lung function clusters have been generated. ${ }^{11}$ Viscovery automatically identified patient characteristics that differ significantly from the average of the whole study sample using the integrated two-sided $t$ test, with a confidence of $95 \%{ }^{11}$

Simultaneously, the efficacy of the pulmonary rehabilitation program was evaluated for each cluster based on the minimal clinically important difference (MCID). The following MCIDs were used: -1 grade on MRC dyspnea scale ${ }^{16} ;+30 \mathrm{~m}$ on 6 -minute walk distance $(6 \mathrm{MWD})^{17,18} ;+100 \mathrm{~s}$ on cycle endurance time $(\mathrm{CWRT})^{19} ;+2$ points on Canadian Occupational Performance Measure, performance (COPM-P) ${ }^{20} ;+2$ points on Canadian Occupational Performance Measure, satisfaction (COPM-S) ${ }^{20} ;-1.5$ points on Hospital Anxiety and Depression Scale, anxiety (HADS-A) ${ }^{21}$; -1.5 points on Hospital Anxiety and Depression Scale, depression (HADS-D) $)^{21}$; and -4 points on St George's Respiratory Questionnaire-Total score (SGRQ-T). ${ }^{22}$ For comparing outcomes of the clusters, a $p$-value of $\leq 0.01$ was set as the level of significance.

\section{Results}

A total of 419 of the 518 patients (80.9\%) completed the rehabilitation program. Patients in Cluster 2 showed a significantly higher dropout rate compared to the whole sample (Figure 6.3). In all clusters, clinically relevant outcomes exceeding a MCID at least once were achieved. The mean improvements in the degree of breathlessness, 6-min walk distance, performance of activities of daily life, symptoms of anxiety and depression, and mean improvement in disease specific quality of life were comparable between clusters. Significant differences were only found in Cluster 2, with lower mean improvement in satisfaction with the performance of activities of daily life, and in Cluster 7, with a lower mean improvement in cycle endurance time (Table 6.1). Figure 6.3 illustrates the changes of these different outcomes per lung function cluster. Changes following pulmonary rehabilitation could not be clustered to specific physiomics profiles. Compared to the whole sample, Cluster 7 demonstrated a lower proportion of outcomes exceeding a MCID at least once. 


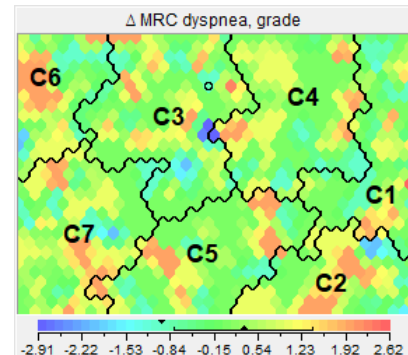

$\begin{array}{lllllllllll}-2.91 & -2.22 & -1.53 & -0.84 & -0.15 & 0.54 & 1.23 & 1.92 & 2.62\end{array}$
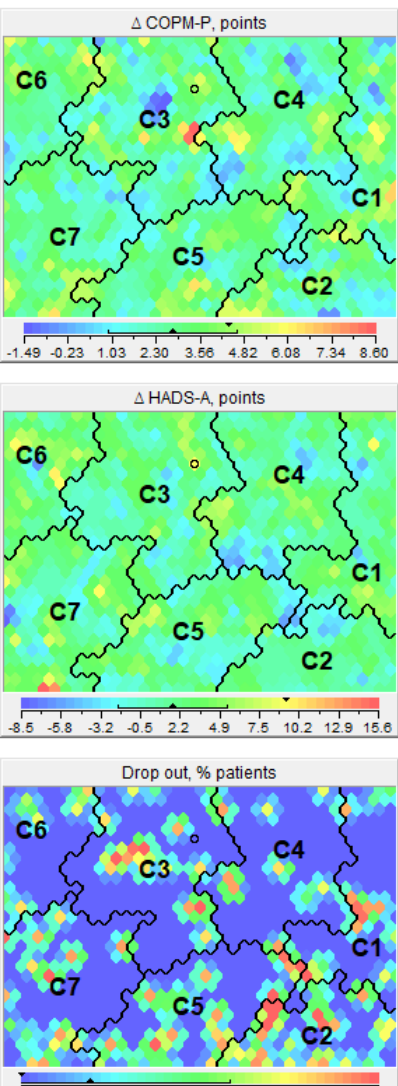

$0.000 .110220 .33 \quad 0.440 .580 .87 \quad 0.780 .89100$
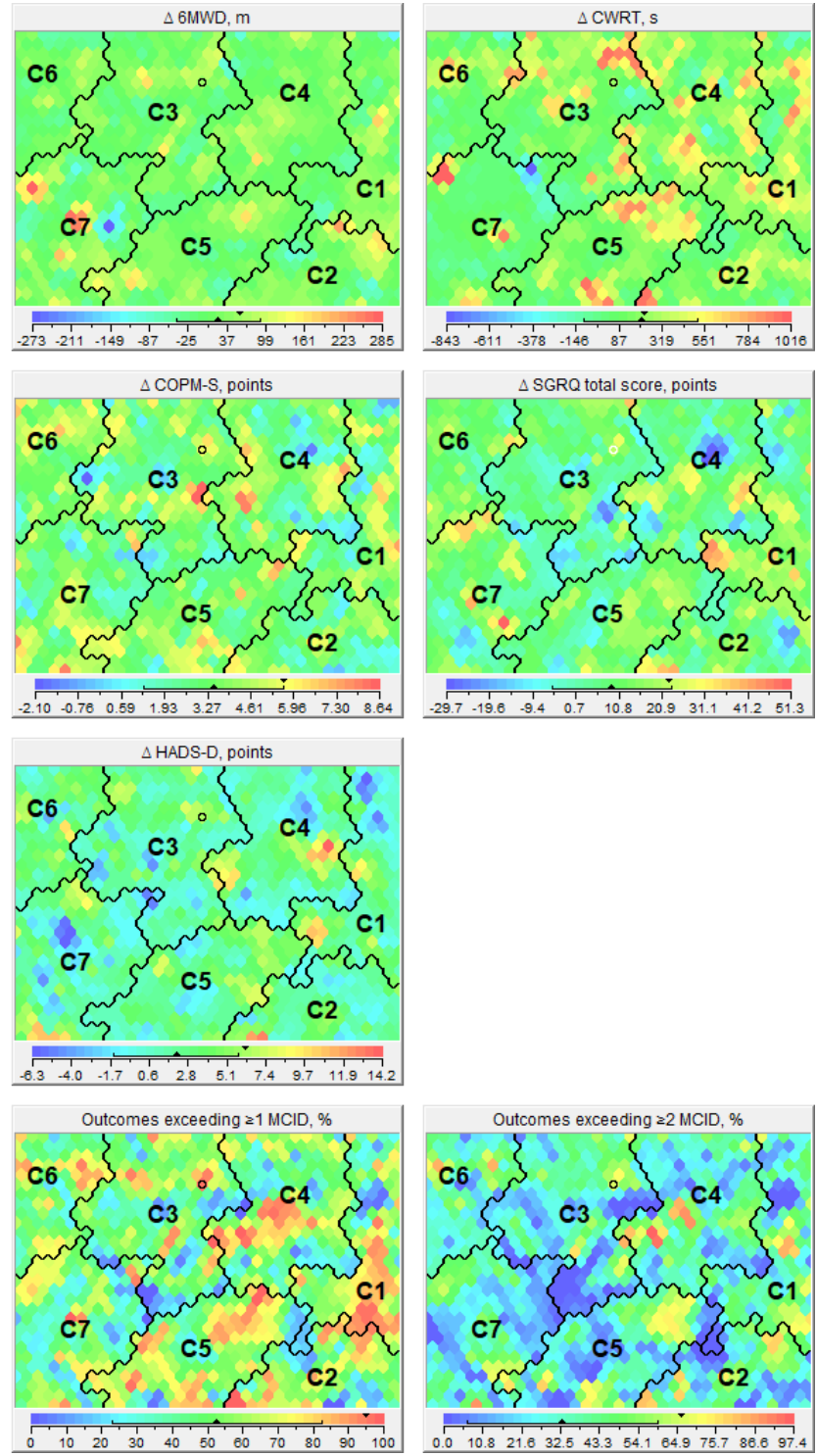

Figure 6.3 Changes following pulmonary rehabilitation. Different panels illustrating the absolute change in Medical Research Council (MRC) dyspnea grade, 6-min walk distance (6MWD), cycle endurance time (constant work-rate test; CWRT), Canadian Occupational Performance Measure, performance (COPM-P), Canadian Occupational Performance Measure, satisfaction (COPM-S), Hospital Anxiety and Depression Scale, anxiety (HADS-A), Hospital Anxiety and Depression Scale, depression (HADS-D), and St. George's Respiratory Questionnaire total score (SGRQ-T) for the seven lung function clusters. The other three panels demonstrate the proportion of patients not completing the pulmonary rehabilitation program, the proportion of clinically relevant outcomes (exceeding at least one minimal clinically important difference (MCID) and the proportion of clinically relevant outcomes (exceeding at least two MCID) for each lung function cluster. 


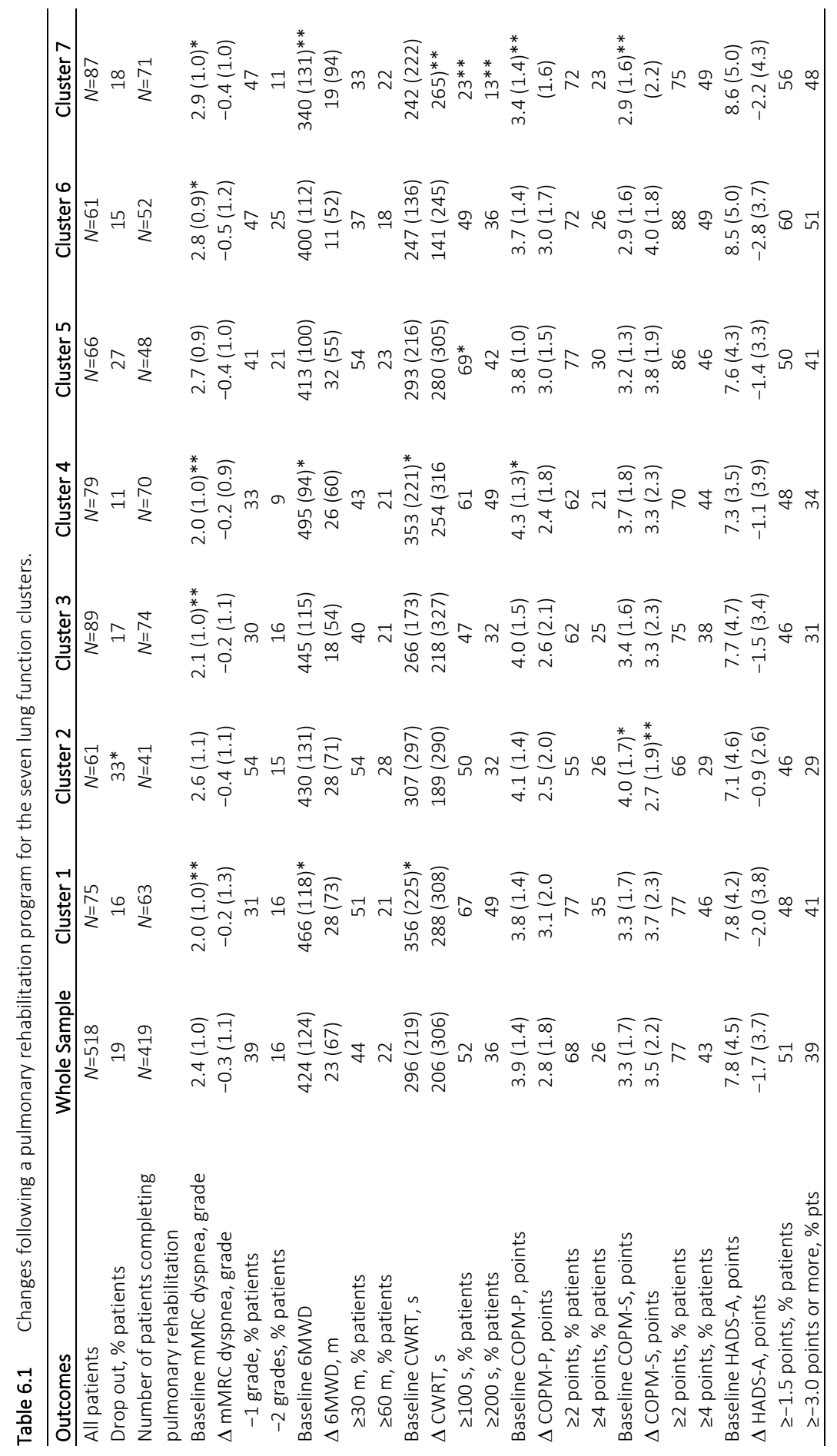




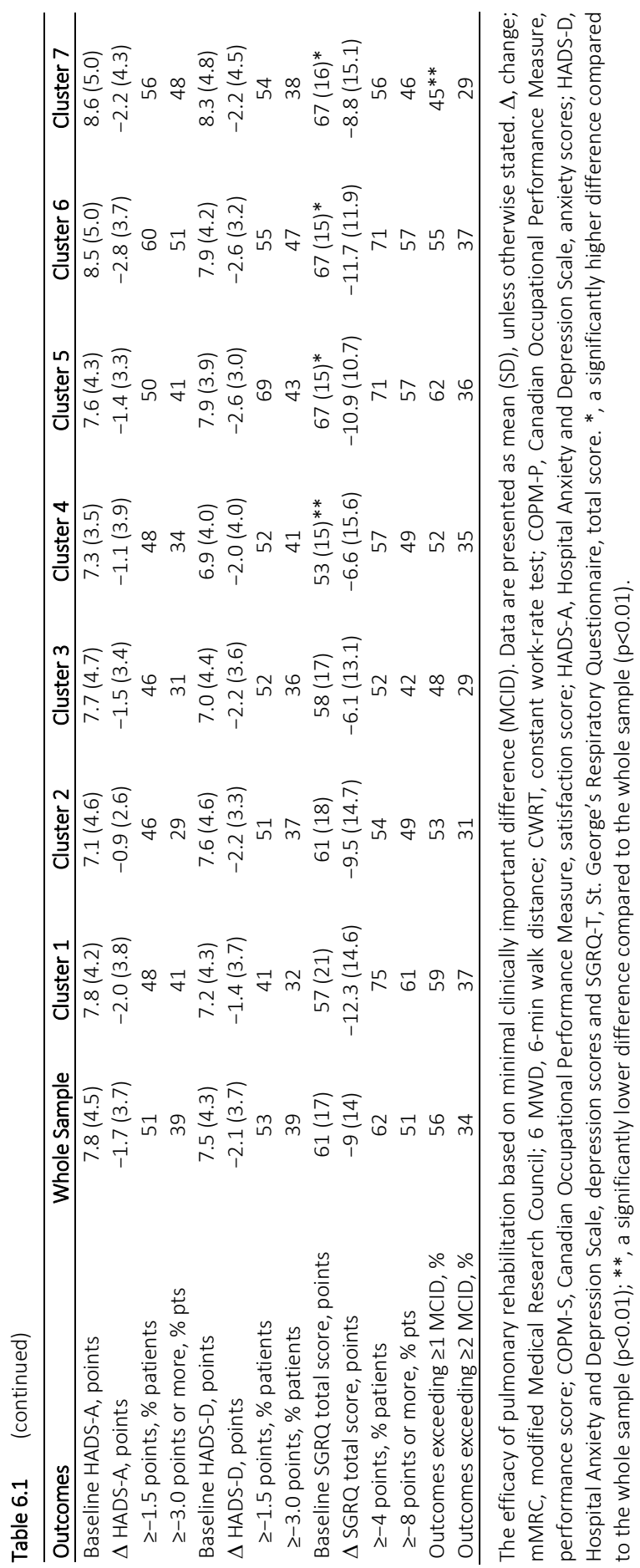




\section{Discussion}

This is the first report on the efficacy of pulmonary rehabilitation in patients with COPD after clustering for a comprehensive lung function assessment. The results demonstrate that the degree of baseline lung function poorly predicts individual improvements in breathlessness, exercise performance, problematic activities of daily living, mood status and disease-specific health status following pulmonary rehabilitation. Even in those with the most severe respiratory impairment (i.e., Clusters 6 and 7), clinically relevant improvements were achieved. Nevertheless, one-third of the patients in Cluster 2 did not complete the program. Why patients within this cluster seem more at risk for dropout is currently unknown and needs further evaluation.

Based on 65 randomized clinical trials involving 3822 patients for inclusion in the metaanalysis, McCarthy and colleagues concluded that pulmonary rehabilitation relieves dyspnea and fatigue, improves emotional function and enhances the sense of control that individuals have over their condition. Moreover, pulmonary rehabilitation is beneficial in improving health status and exercise capacity. ${ }^{2}$ Our study confirms that improvements following pulmonary rehabilitation are clinically relevant and statistically significant. ${ }^{2,3}$ According to McCarthy and colleagues, additional RCTs comparing pulmonary rehabilitation with standard COPD care are no longer warranted. ${ }^{2}$ In order to improve outcomes, identification of markers predicting outcomes in individual patients could be very interesting. At the very least, our study illustrates that even a comprehensive lung function assessment is unhelpful in achieving this goal. Alternatively, cluster analysis could be helpful to implement specific interventions such as inspiratory muscle training in those COPD patients with respiratory muscle dysfunction but without static hyperinflation. ${ }^{11}$

Since quality of life is determined by the degree of dyspnea, depression, anxiety and exercise performance ${ }^{23}$, these factors should be taken into consideration in personalizing the intervention. Furthermore, as pulmonary rehabilitation programs change their emphasis towards the ability to adapt and self-manage in the face of social, physical and emotional challenges, traditional disease-related characteristics of disease severity are no longer dominant. ${ }^{24}$ The importance of understanding the unique circumstances of the individual is now widely accepted but still neglected in pulmonary rehabilitation. The patient's health beliefs, the way illness is approached, as well as the interactions of the patient with the medical system are affected by social, psychological, cultural, behavioral and economic factors. These unique circumstances or personomics should be considered in order to understand the patient's preferences, values and goals. $^{25}$

Our study confirms that a comprehensive pulmonary rehabilitation program results in a heterogeneous and differential pattern of patient-related outcomes. This confirms our 
previous study, that a multidimensional response needs to be considered to evaluate the efficacy of pulmonary rehabilitation services. ${ }^{3}$ Furthermore, the differential response pattern, the non-linear responses as well as the absent or poor response illustrate that a "one size fits all" approach is no longer applicable in pulmonary rehabilitation. In addition, non-linear responses as well as unpredictability in response must be considered as a reflection of the intrinsic complexity of the patient themselves. ${ }^{26}$

Pulmonary rehabilitation requires multidimensional profiling of patients, not restricted to pathophysiological respiratory system involvement. Future identification of essential components of pulmonary rehabilitation should be based on a personomic perspective. ${ }^{25}$ Comprehensive intervention can no longer be based on restoration of impairments, it needs to become person-centered.

\section{Conclusion}

The current study demonstrates no relationship between the seven lung-function-based clusters and response to pulmonary rehabilitation in patients with COPD. Therefore, baseline lung function cannot be used to identify good responders to pulmonary rehabilitation, and therefore, cannot be used as a criterion for referral to pulmonary rehabilitation in patients with COPD. 


\section{References}

1. Spruit MA, Singh SJ, Garvey C, ZuWallack R, Nici L, Rochester C, Hill K, Holland AE, Lareau SC, Man WD, et al. An official American Thoracic Society/European Respiratory Society statement: Key concepts and advances in pulmonary rehabilitation. Am J Respir Crit Care Med 2013;188:e13-e64.

2. McCarthy B, Casey D, Devane D, Murphy K, Murphy E, Lacasse Y. Pulmonary rehabilitation for chronic obstructive pulmonary disease. Cochrane Database Syst Rev. 2015, volume, CD003793.

3. Spruit MA, Augustin IM, Vanfleteren LE, Janssen DJ, Gaffron S, Pennings HJ, Smeenk F, Pieters W, van den Bergh JJ, Michels AJ, et al. Differential response to pulmonary rehabilitation in COPD: Multidimensional profiling. Eur Respir J. 2015;46:1625-35.

4. Vaes AW, Delbressine JML, Mesquita R, Goertz YMJ, Janssen DJA, Nakken N, Franssen FME, Vanfleteren L, Wouters EFM, Spruit MA. The impact of pulmonary rehabilitation on activities of daily living in patients with COPD. J Appl Physiol. 2018, doi:10.1152/japplphysiol.00790.2018.

5. Annegarn J, Meijer K, Passos VL, Stute K, Wiechert J, Savelberg HH,; Schols AM, Wouters EF, Spruit MA. Problematic activities of daily life are weakly associated with clinical characteristics in COPD. J Am. Med Dir Assoc. 2012;13:284-90.

6. Agusti A, Calverley PM, Celli B, Coxson HO, Edwards LD, Lomas DA, MacNee W, Miller BE, Rennard S, Silverman EK, et al. Characterisation of COPD heterogeneity in the ECLIPSE cohort. Respir Res. 2010;11:122.

7. Spruit MA, Watkins ML, Edwards LD, Vestbo J, Calverley PM, Pinto-Plata V, Celli BR, Tal-Singer R, Wouters EF. Determinants of poor 6-min walking distance in patients with COPD: The ECLIPSE cohort. Respir Med. 2010;104:849-57.

8. Vogiatzis I, Williamson AF, Miles J, Taylor IK. Physiological response to moderate exercise workloads in a pulmonary rehabilitation program in patients with varying degrees of airflow obstruction. Chest. 1999; 116:1200-7.

9. Vogiatzis I, Terzis G, Stratakos G, Cherouveim E, Athanasopoulos D, Spetsioti S, Nasis I, Manta P, Roussos C, Zakynthinos S. Effect of pulmonary rehabilitation on peripheral muscle fiber remodeling in patients with COPD in GOLD stages II to IV. Chest. 2011;140:744-52.

10. Vanfleteren MJ, Koopman M, Spruit MA, Pennings HJ, Smeenk F, Pieters W, van den Bergh JJ, Michels AJ, Wouters EF, Groenen MT, et al. Effectiveness of Pulmonary Rehabilitation in Patients With Chronic Obstructive Pulmonary Disease With Different Degrees of Static Lung Hyperinflation. Arch Phys Med Rehabil. 2018;99:2279-86 e3.

11. Augustin IML, Spruit MA, Houben-Wilke S, Franssen FME, Vanfleteren L, Gaffron S, Janssen DJA, Wouters EFM. The respiratory physiome: Clustering based on a comprehensive lung function assessment in patients with COPD. PLOS ONE. 2018;13:e0201593.

12. Smid DE, Wilke S, Jones PW, Muris JW, Wouters EF, Franssen FM, Spruit MA. Impact of cardiovascular comorbidities on COPD Assessment Test. (CAT) and its responsiveness to pulmonary rehabilitation in patients with moderate to very severe COPD: Protocol of the Chance study. BMJ Open 2015;5:e007536.

13. Clinical, Physiological and Psychosocial Determinants of the COPD Assessment Test (CAT). Available online: http://www.trialregister.nl (accessed on 26 December 2018).

14. Augustin IML, Spruit MA, Franssen FME, Wouters EFM. Understanding Complexity of Chronic NonCommunicable Diseases: An. Integrated Approach for Personalized Management of Patients with COPD. Clin Res Pulmonol. 2015;3:1034.

15. Available online: https://www.viscovery.net (accessed on 26 December 2018)

16. de Torres JP, Pinto-Plata V, Ingenito E, Bagley P, Gray A, Berger R, Celli B. Power of outcome measurements to detect clinically significant changes in pulmonary rehabilitation of patients with COPD. Chest. 2002;121:1092-8.

17. Holland AE, Spruit MA, Troosters T, Puhan MA, Pepin V, Saey D, McCormack MC, Carlin BW, Sciurba FC, Pitta F, et al. An official European Respiratory Society/American Thoracic Society technical standard: Field walking tests in chronic respiratory disease. Eur Respir J. 2014;44:1428-46.

18. Singh SJ, Puhan MA, Andrianopoulos V, Hernandes NA, Mitchell KE, Hill CJ, Lee AL, Camillo CA, Troosters $T$, Spruit MA, et al. An official systematic review of the European Respiratory Society/American Thoracic Society: Measurement properties of field walking tests in chronic respiratory disease. Eur Respir J. 2014; 44:1447-78. 
19. Laviolette L, Bourbeau J, Bernard S, Lacasse Y, Pepin V, Breton MJ, Baltzan M, Rouleau M, Maltais F. Assessing the impact of pulmonary rehabilitation on functional status in COPD. Thorax. 2008;63:115-21.

20. Sewell L, Singh SJ. The Canadian Occupational Performance Measure: Is it a Reliable Measure in Clients with Chronic Obstructive Pulmonary Disease? Br J Occup Ther. 2001;64:305-10.

21. Puhan MA, Frey M, Buchi S, Schunemann HJ. The minimal important difference of the hospital anxiety and depression scale in patients with chronic obstructive pulmonary disease. Health Qual. Life Outcomes. 2008;6:46.

22. Jones PW. Interpreting thresholds for a clinically significant change in health status in asthma and COPD. Eur Respir J. 2002;19:398-404.

23. Tsiligianni I, Kocks J, Tzanakis N, Siafakas N, van der Molen T. Factors that influence disease-specific quality of life or health status in patients with COPD: A review and meta-analysis of Pearson correlations. Prim Care Respir J. 2011;20:257-68.

24. Huber M, Knottnerus JA, Green L, van der Horst H, Jadad AR, Kromhout D, Leonard B, Lorig K, Loureiro MI, van der Meer JW, et al. How should we define health? BMJ 2011, 343, d4163.

25. Ziegelstein RC. Personomics. JAMA Int Med. 2015;175:888-9.

26. Wilson T, Holt T, Greenhalgh T. Complexity science: Complexity and clinical care. BMJ. 2001;323:685-8. 




\section{Chapter

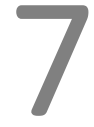

Incorporating comprehensive assessment parameters to better characterize and plan rehabilitation for persons with COPD

Ingrid ML Augustin, Martijn A Spruit, Frits ME Franssen, Swetlana Gaffron, Frits van Merode, EFM Wouters Journal of American Medical Directors Association 2020;21(12):1986-1991.e3. 


\section{Abstract}

\section{Objectives}

The current management of chronic obstructive pulmonary disease (COPD) largely ignores its heterogeneous pulmonary and extra-pulmonary manifestations in the individual patient. This study aimed to identify clusters of patients with COPD based on a thorough traits assessment.

\section{Design}

An observational, prospective, single-center study.

\section{Setting and participants}

Patients with COPD referred by chest physicians for a comprehensive pulmonary rehabilitation program to Ciro (Horn, the Netherlands) were eligible to participate. Ciro is a specialized pulmonary rehabilitation center in the southern part of the Netherlands for patients suffering from complex underlying respiratory diseases.

\section{Methods}

Clinically stable patients with COPD underwent a comprehensive assessment including pulmonary traits (airflow limitation, static hyperinflation, gas transfer, respiratory pressures and arterial blood gases), extra-pulmonary functional traits and health status (quadriceps muscle strength, physical functioning, body composition, comorbidities, symptoms perception, and social and emotional functioning). Clusters were generated using the SOM-Ward Cluster algorithm, a hybrid algorithm that applies the classical hierarchical method of Ward on top of the SOM topology.

\section{Results}

Based on the abovementioned attributes of 518 patients with mild to very severe COPD (44\% women, age $64.1 \pm 9.1$ years, forced expiratory volume in the first second $48.6 \% \pm 20.0 \%$ of predicted), 7 clusters were identified. Clusters had unique patterns differing in demographics, pulmonary, extra-pulmonary functional and behavioral traits and/or health status.

\section{Conclusion and implications}

The tremendous heterogeneity in pulmonary, extra-pulmonary functional and behavioral traits, and health status in COPD patients supports the need for an individual comprehensive assessment and a goal-directed personalized management strategy. 


\section{Introduction}

The rise in life expectancy worldwide is accompanied by an increased incidence of agerelated diseases. The lungs normally ages with a progressive decline in pulmonary function after the age of about 25 years and this aging is associated with a progressive functional impairment and reduced capacity to respond to environmental stresses and injury. ${ }^{1}$ Chronic Obstructive Pulmonary Disease (COPD) shows striking age - associated features such as cellular senescence and evidence of accelerated ageing of the lungs., ${ }^{2,3}$ In addition, premature cellular senescence and subsequent exhaustion of muscle regenerative potential seem related to the muscle abnormalities in these patients. ${ }^{4}$ Mounting evidence supports that COPD is a clinical syndrome driven by different underlying processes as accelerated ageing. ${ }^{5}$

Current management of COPD is largely based on results obtained from pharmacological trials. ${ }^{6}$ This one-size-fits-all approach largely ignores the heterogeneous clinical manifestation in the individual patient. As a consequence, a moderate-to-high disease burden still persists in a large group of COPD patients despite current pharmacological therapy. ${ }^{7}$

Identification of pulmonary and extra-pulmonary treatable traits has been proposed as a way towards a more personalized treatment strategy for these patients. ${ }^{8}$ It is assumed that recognition of this clinical complexity in patients with COPD paves the way to a more precise and more effective individualized therapy.

Such a patient-tailored targeted management program, based on a thorough patient assessment forms the cornerstone of a comprehensive pulmonary rehabilitation program. ${ }^{9,10}$ Indeed, extra-pulmonary functional traits commonly evaluated in geriatric medicine (i.e., mobility/balance testing using the Time Up and Go [TUG] test, quadriceps muscle strength, fat-free mass, osteoporosis, exercise capacity using the 6-min walk distance and problematic activities of daily living $[A D L])$, and behavioral or emotional traits (i.e., symptoms of anxiety and depression, activity-related dyspnea, care dependency and health status) are all assessed to characterize the patients beyond the lungs. ${ }^{11,12}$

Clustering of various sets of clinical variables has been used to identify COPD subtypes. ${ }^{13,14}$ In a recent study, we reported the heterogeneity in respiratory physiomics and its poor relationship with functional performance, symptoms of anxiety and depression, and health status in patients with COPD. ${ }^{15}$

As previous cluster analyses were based on limited sets of variables, this study aims to identify clusters of pulmonary and extra-pulmonary traits experienced by these disabled patients with COPD. We hypothesized that identification of specific clusters could help 
guide the organizational structure and the interdisciplinary team composition of pulmonary rehabilitation programs and to help design, implement, and ensure adherence of long-term care in these patients.

\section{Materials and methods}

\section{Study design}

The current analyses are based on the data from the Chance Study: an observational, prospective, single-center study about COPD, health status, and cardiovascular comorbidities in relation to the outcomes of pulmonary rehabilitation. ${ }^{16}$ This study was approved by the Medical Ethical Committee of the Maastricht University Medical Centre+ (METC 11-3-070) and is registered at http://www.trialregister.nl (NTR 3416).

\section{Study sample}

Patients with COPD referred by chest physicians from general and academic hospitals for a comprehensive pulmonary rehabilitation program to Ciro (Horn, the Netherlands) were eligible to participate. Ciro is a specialized pulmonary rehabilitation center in the southern part of the Netherlands for patients suffering from complex underlying respiratory diseases. All patients gave written informed consent.

\section{Measurements}

All patients underwent an integrated baseline assessment, reflecting the degree of complexity of COPD. ${ }^{16}$ Pulmonary and extra-pulmonary (functional, behavioral and health status) attributes were identified and categorized ${ }^{8,17}$ as illustrated in Table 7.1.

Pulmonary traits were determined by a comprehensive lung function assessment as described previously ${ }^{15}$ and existed of airflow limitation, static hyperinflation, gas transfer, respiratory pressures and arterial blood gases. Post-bronchodilator forced expiratory volume in the first second $\left(F E V_{1}\right)$, forced vital capacity $(F V C), F E V_{1} / F V C$, peak expiratory flow (PEF), total lung capacity (TLC), residual volume (RV), intra thoracic gas volume (ITGV), diffusing capacity of carbon monoxide (TLCO), TLCO per unit alveolar volume (KCO), maximal static inspiratory (MIP) and expiratory mouth pressures (MEP) were measured. Furthermore, resting arterial partial pressure of oxygen $\left(\mathrm{PaO}_{2}\right)$, carbon dioxide $\left(\mathrm{PaCO}_{2}\right)$ and oxygen saturation $\left(\mathrm{SaO}_{2}\right)$ were determined. Patients with long term oxygen therapy (LTOT) continued oxygen supply during the procedure. All lung function measurements were performed by certified and experienced respiratory technicians.

Measured functional extra-pulmonary traits (physical) were muscle strength, physical functioning, body composition and presence of comorbidities. Isokinetic quadriceps peak torque was measured using a Biodex (Biodex Medical Systems, Inc., New York, USA). ${ }^{18,19}$ Exercise performance was assessed by a 6 -minute walk test (6MWT) 
performed according to international guidelines. ${ }^{20}$ The longest distance covered in 2 separate tests was registered as the 6-minute walk distance (6MWD). Furthermore, a symptom-limited incremental cardiopulmonary exercise test (CPET) was performed using an electrically, braked cycle ergometer (Carefusion, Houten, the Netherlands) including the measurement of maximal oxygen uptake (Peak $\mathrm{VO}_{2}, \mathrm{ml} / \mathrm{min}$ ) and maximal work rate in Watts (peak work rate). ${ }^{21}$ Endurance exercise capacity was measured by a sub-maximal exercise test at $75 \%$ of the peak work rate (i.e., Constant work-rate test [CWRT]). ${ }^{22}$ Body mass index (BMI) was calculated by body weight (in kg) divided by squared height (in $\mathrm{m}$ ). Fat-free mass (FFM) was assessed using dual-energy X-ray absorptiometry (DEXA) scan (Lunar Prodigy scan, GE Healthcare, Madison, WI, USA) and FFM (lean mass + bone mineral content) was divided by squared height (in $\mathrm{m}$ ) to obtain the FFM-index (FFMI). ${ }^{23}$ Bone mineral density (BMD by DEXA scan) was measured at the hip, lumbar spine, and whole body. ${ }^{24}$ Self-reported comorbidities were assessed using the Charlson Comorbidity Index (CCl). ${ }^{25}$

Extra-pulmonary behavioral traits reflected by social functioning and emotional function, symptom perception and health status were assessed. The Care Dependency Scale (CDS), consisting of 15 items regarding basic and instrumental activities of daily living, was used to assess the level of care dependency. ${ }^{26}$ The Canadian Occupational Performance Measure (COPM) was used to identify specific problematic activities of daily life. ${ }^{27}$ Patients scored how well they were performing the problematic activities of daily life (performance score; COPM-P) and how satisfied they were with this level of performance (satisfaction score; COPM-S). Timed Up and Go test (TUG) test was assessed to measure functional mobility. ${ }^{28}$ Emotional functioning has been measured using the Hospital Anxiety and Depression Scale (HADS) ${ }^{29}$, which is divided into an anxiety subscale (HADS-A) and a depression subscale (HADS-D). The degree of dyspnea was measured using the modified Medical Research Council (mMRC) scale. ${ }^{30}$ The disease-specific health status was assessed using the COPD Assessment Test (CAT) ${ }^{31}$, a simple questionnaire to measure the impact of COPD on a person's life, and using the COPD-specific version of the St George's Respiratory Questionnaire (SGRQ-C) ${ }^{32}$ that consists of 3 domains scores (symptoms, activity and impact) and a total score. Furthermore, the Clinical COPD Questionnaire (CCQ), a self-administered questionnaire consisting of 3 domains specially developed to measure clinical control in patients with COPD, was used. ${ }^{33}$

In addition the assessment included demographics, medical history, the number of exacerbations and hospitalizations for COPD in the last 12 months, and the use of longterm oxygen (LTOT). Patients with COPD were classified as GOLD I to IV, and GOLD A to $D$, according the latest GOLD strategy. ${ }^{6}$ 
Table 7.1 Treatable traits.

\begin{tabular}{|c|c|}
\hline \multicolumn{2}{|l|}{ Pulmonary } \\
\hline \multirow[t]{4}{*}{ Airflow limitation } & Forced expiratory volume in 1 second $\left(\mathrm{FEV}_{1}\right)$ \\
\hline & Forced vital capacity (FVC) \\
\hline & $\mathrm{FEV}_{1} / \mathrm{FVC}$ \\
\hline & Peak expiratory flow (PEF) \\
\hline \multirow[t]{2}{*}{ Gas transfer } & Single-breath transfer factor of the lung for carbon monoxide (TLCO) \\
\hline & TLCO per unit alveolar volume (KCO) \\
\hline \multirow[t]{3}{*}{ Hyperinflation } & Total lung capacity (TLC) \\
\hline & Residual volume (RV) \\
\hline & Intra thoracic gas volume (ITGV) \\
\hline \multirow[t]{2}{*}{ Respiratory pressures } & Maxima static inspiratory mouth pressure (MIP) \\
\hline & Maximal static expiratory mouth pressure (MEP) \\
\hline \multirow[t]{3}{*}{ Arterial blood gases } & Arterial oxygen tension $\left(\mathrm{PaO}_{2}\right)$ \\
\hline & Arterial carbon dioxide tension $\left(\mathrm{PaCO}_{2}\right)$ \\
\hline & Oxygen saturation $\left(\mathrm{SaO}_{2}\right)$ \\
\hline \multicolumn{2}{|c|}{ Extra-pulmonary - functional } \\
\hline Muscle strength & Peak isokinetic quadriceps peak torque \\
\hline \multirow[t]{4}{*}{ Physical functioning } & 6-minute walk distance \\
\hline & $\begin{array}{l}\text { Constant work-rate bicycle test and symptom limited cardiopulmonary } \\
\text { exercise test }\end{array}$ \\
\hline & Timed Up and Go (TUG) \\
\hline & Use of rollator \\
\hline \multirow[t]{3}{*}{ Body composition } & Body mass \\
\hline & Fat-free mass \\
\hline & Bone mineral density \\
\hline Comorbidities & Self-reported comorbidities: Charlson Comorbidity Index (CCI) \\
\hline \multicolumn{2}{|c|}{ Extra-pulmonary - behavioral and health status } \\
\hline \multirow[t]{2}{*}{ Social functioning } & Self-reported care dependency: Care Dependency Scale (CDS) \\
\hline & $\begin{array}{l}\text { Problematic activities of daily life: Canadian Occupational Performance } \\
\text { Measure (COPM) }\end{array}$ \\
\hline Emotional functioning & Anxiety and depression (HADS-A and HADS-D) \\
\hline Symptom perception & Dyspnea score: modified Medical Research Council (mMRC) \\
\hline \multirow[t]{3}{*}{ Health status } & COPD Assessment Test (CAT) \\
\hline & St George's Respiratory Questionnaire (SGRQ) \\
\hline & Clinical COPD Questionnaire (CCQ) \\
\hline
\end{tabular}

\section{Statistics}

Self-organizing maps (SOMs, also referred to as Kohonen maps) were used to create an ordered representation of the selected attributes. The SOM method can be viewed as a non-parametric regression technique that simplifies complexity by converting multidimensional data spaces into lower dimensional abstractions. A SOM generates a non-linear representation of the data distribution and allows the user to identify homogeneous data groups visually to reveal meaningful relationships. All statistical analyses were performed using Viscovery SOMine 7.1 by Viscovery Software GmbH (http://www.viscovery.net, Vienna, Austria).

Patients have been ordered by their overall similarity concerning the attributes in Table 7.1, which are the following in descending order of priority: $\mathrm{FEV}_{1} / \mathrm{FVC}$ in \%; FEV $1, F V C$, 
PEF, ITGV, RV,TLC TLCO, KCO, MIP and MEP (all in \% predicted); mMRC dyspnea grade; CAT, total score; SGRQ, total score; $C C Q$, total score; CDS, item daily activities in points and CDS, total points; COPM-P in points; 6MWD in $\mathrm{m}$; Peak work rate in Watts; Quadriceps peak torque in \% predicted; TUG test in $\mathrm{s}$; FEV 1 in L; Airway resistance effective measured during bodyplethysmography in KPa*sec/L; COPD GOLD I-IV; HADS-D in points; Users of rollator in \%; HADS-A in points; CAT score section impact on activities; CDS, item mobility; 6MWD in \% predicted; LTOT users in \%; CDS, item eating and drinking in points; CDS, item getting dressed and undressed in points; Quadriceps muscle endurance (Total Work in Joules); SGRQ score, section activity in points; SGRQ score, for section impact in points; COPD related hospital admissions last 12 months; SGRQ score, section symptom in points; arterial blood gases $\left(\mathrm{PaCO}_{2}, \mathrm{CHCO}_{3}, \mathrm{PaO}_{2}, \mathrm{SaO}_{2}\right)$ and $\mathrm{CCQ}$ scores for functional state; and to a small extent absolute measures of FVC; PEF; ITGV; RV; TLC; TLCO; KCO; TLC by helium dilution technique; Inspiratory vital capacity (IC); breath holding time (TA); MIP; and MEP.

Based on the created SOM model, clusters have been generated using the SOM-Ward Cluster algorithm of Viscovery, a hybrid algorithm that applies the classical hierarchical method of Ward on top of the SOM topology. Summary variables on clinical characteristics for the total sample and for each cluster are presented in all tables as mean \pm standard deviation for quantitative variables, and percentage for discrete variables. Viscovery automatically identified for each cluster all patient characteristics that differ significantly from the average of the whole study sample using the integrated two-sided t-test with a confidence of $99 \%$.

\section{Results}

\section{Patient characteristics}

A total of 518 patients was analyzed (Table 7.2). The majority of patients had COPD GOLD D (72\%). Patients had a substantial smoking history, and a high disease burden and medical resource use reflected by the mean number of exacerbations as well as hospitalizations in the last year (2.2 and 0.9, respectively). A quarter of them used LTOT. On average, patients had an impaired physical fitness, one or more comorbidities and experienced a high impact on activities in daily life.

\section{Patient profiles of the 7 clusters}

Seven clusters were identified (Figure 7.1). Demographics and clinical characteristics are summarized in Table 7.3. Cluster 3 (the multi-morbid cluster) included the oldest patient group; and the proportion of women was lowest in cluster 1 (the overall best functioning cluster). Exacerbations in the last 12 months and COPD related hospital admissions were 
significantly lower in clusters 1 and 4 (the low burden cluster) and higher in cluster 6 (the overall worst-functioning cluster). The proportion of patients using LTOT was significant lower in clusters 1, 2 (the ADL most limited cluster) and 4, whereas it was higher in clusters 3 and 6 . The number of medications and different kind of medications were most prominent in cluster 6 .

Clusters 1, 2 and 3 included patients with a significantly lower degree of airflow limitation than clusters 6 and 7 (the physically dysfunctioning cluster). Static hyperinflation was particularly present in clusters 4, 5 (the emotionally dysfunctioning cluster), 6 and 7. Clusters 3 to 7 demonstrated an impaired diffusion capacity: respiratory inspiratory muscle functioning was lower in clusters 3 and 6 with preservation of respiratory muscle strength in cluster 4 and 5 despite presence of static hyperinflation. $\mathrm{PaO}_{2}$ levels were well preserved in all patients, whereas only cluster 6 demonstrated patients with alveolar hypoventilation.

Patients with the highest values for quadriceps muscle function, 6-minute walk distance, maximal exercise test and constant work rate test were classified in cluster 1 , whereas patients with the lowest quadriceps muscle function and very poor physical performance were found in cluster 6 . Clusters 2, 4 and 5 had an intermediate impairment in physical performance, whereas cluster 3 had a manifested skeletal muscle weakness, as reflected in severe impairment in different exercise measurements and highest Timed Up and Go test. Remarkably, clusters 2 and 3 included patients with a high number of comorbidities. Compared with all the other clusters, patients in cluster 7 had the lowest body mass index and fat-free mass index, with poor physical performance and quadriceps muscle function.

Care dependency was significantly high in cluster 6 and low in cluster 4 . Patients in clusters 2 and 6 experienced the highest limitations in daily activities. Mood problems are particularly present in clusters 2, 5 and 6, with the highest scores for anxiety and depression in cluster 6 . Dyspnea was significantly higher in clusters 3 and 6 and lower in clusters 1 and 4 . Health status was significantly worse in clusters 2, 5, and 6, and better in clusters 1 and 4.

Figure 7.2 illustrates the distribution of individual traits across the 7 clusters, reflecting the tremendous heterogeneity of individual traits in our COPD sample. 
Table 7.2 Demographics, clinical characteristics, pulmonary, extra-pulmonary, behavioral and health status of the whole sample.

\begin{tabular}{|c|c|}
\hline & $\begin{array}{c}\text { Whole sample } \\
n=518\end{array}$ \\
\hline \multicolumn{2}{|l|}{ Demographics and clinical characteristics } \\
\hline Women, \% & 44 \\
\hline Age, years & $64.1(9.1)$ \\
\hline Smoking pack years & $42.4(23.6)$ \\
\hline Exacerbations $<1$ year, $\mathrm{n}$ & $2.2(1.8)$ \\
\hline Hospitalizations $<1$ year, $\mathrm{n}$ & $0.9(1.3)$ \\
\hline Patients with long-term oxygen therapy (LTOT) use, \% & 24.1 \\
\hline Number of medications, $\mathrm{n}$ & $7.2(3.6)$ \\
\hline Number of different kind of medications, $n$ & $6.8(3.2)$ \\
\hline Patients with GOLD I / II /III / IV , \% & $7 / 36 / 37 / 20$ \\
\hline Patients with GOLD A / B / C / D , \% & $3 / 20 / 5 / 72$ \\
\hline \multicolumn{2}{|l|}{ Pulmonary traits } \\
\hline Forced expiratory volume in the first second $\left(\mathrm{FEV}_{1}\right), \%$ predicted & $48.6(20)$ \\
\hline $\mathrm{FEV}_{1} / \mathrm{FVC}, \%$ & $37.5(12.2)$ \\
\hline Intra thoracic gas volume (ITGV), \% of predicted & $148.6(35.9)$ \\
\hline Total lung capacity (TLC), \% of predicted & $117.1(17.5)$ \\
\hline Transfer factor of the lung for carbon monoxide (TLCO), \% of predicted & $49.3(17.2)$ \\
\hline Maximal inspiratory pressure (MIP), \% of predicted & $78.5(23.3)$ \\
\hline Maximal expiratory pressure (MEP), \% of predicted & $63.2(20.4)$ \\
\hline Partial arterial oxygen pressure $\left(\mathrm{PaO}_{2}\right), \mathrm{kPa}$ & $5.3(0.9)$ \\
\hline Partial arterial carbon dioxide $\left(\mathrm{PaCO}_{2}\right), \mathrm{kPa}$ & $9.5(1.5)$ \\
\hline Oxygen saturation $\left(\mathrm{SaO}_{2}\right), \%$ & $93.9(3.2)$ \\
\hline \multicolumn{2}{|l|}{ Extra-pulmonary traits - physical } \\
\hline Quadriceps peak torque, $\%$ of predicted & $66.2(18.9)$ \\
\hline Six-minute walk distance (6MWD), meters & $424(124.4)$ \\
\hline Peak work rate, $\%$ predicted & $55.5(27.4)$ \\
\hline Peak aerobic capacity $\left(\mathrm{VO}_{2}\right), \%$ of predicted & $66.2(30.4)$ \\
\hline Constant work-rate test (CWRT), seconds & $295.5(218.7)$ \\
\hline Timed Up and Go (TUG) test, seconds & $10.6(3.4)$ \\
\hline Use of rollator, \% & 29 \\
\hline Body mass index (BMI), kg/m² & $26.2(5.8)$ \\
\hline Fat-free mass index (FFMI), $\mathrm{kg} / \mathrm{m}^{2}$ & $17.2(2.6)$ \\
\hline Charlson's Comorbidity Index (CCl), points & $1.6(1.0)$ \\
\hline \multicolumn{2}{|l|}{ Extra-pulmonary traits - behavioral and health status } \\
\hline Care Dependency Scale (CDS), points & $69(7)$ \\
\hline Canadian Occupational Performance Measure (COPM) performance, points & $3.9(1.4)$ \\
\hline COPM satisfaction, points & $3.3(1.7)$ \\
\hline Hospital Anxiety and Depression Scale (HADS) anxiety, points & $7.8(4.5)$ \\
\hline HADS depression, points & $7.5(4.3)$ \\
\hline Modified Medical Research Council (mMRC) dyspnea grade & $2.4(1.0)$ \\
\hline COPD Assessment Test (CAT), total score, points & $21.5(6.6)$ \\
\hline St. George's Respiratory Questionnaire (SGRQ), total score, points & $61.1(17.4)$ \\
\hline COPD Clinical Questionnaire (CCQ), total score, points & $2.6(1.0)$ \\
\hline
\end{tabular}




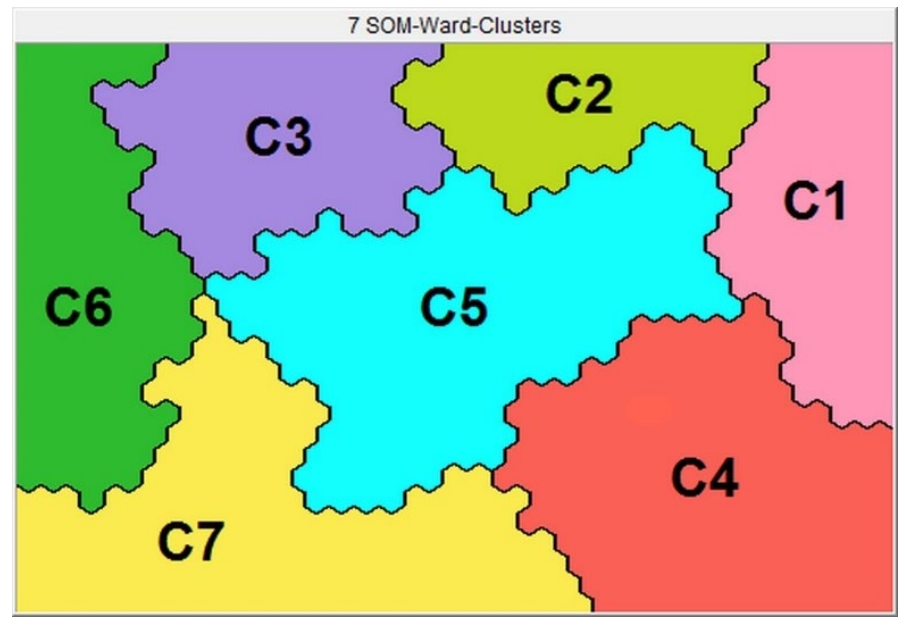

Figure 7.1 Seven different clusters. The Viscovery SOMine program placed all patients with COPD on a specific position of the map based on their baseline characteristics. Subjects located close to each other on the map resemble in terms of their baseline characteristics. Based on the SOM model created, seven SOM-Ward clusters with significantly different profiles have been generated: $\mathrm{C} 1$, 'the overall best functioning' cluster; $\mathrm{C2}$, 'the ADL-limited' cluster; $\mathrm{C} 3$, 'the multimorbid' cluster; C4, 'the low burden' cluster; C5, 'the emotionally dysfunctioning' cluster; C6, 'the overall worst functioning' cluster and C7, 'the physically dysfunctioning' cluster. 


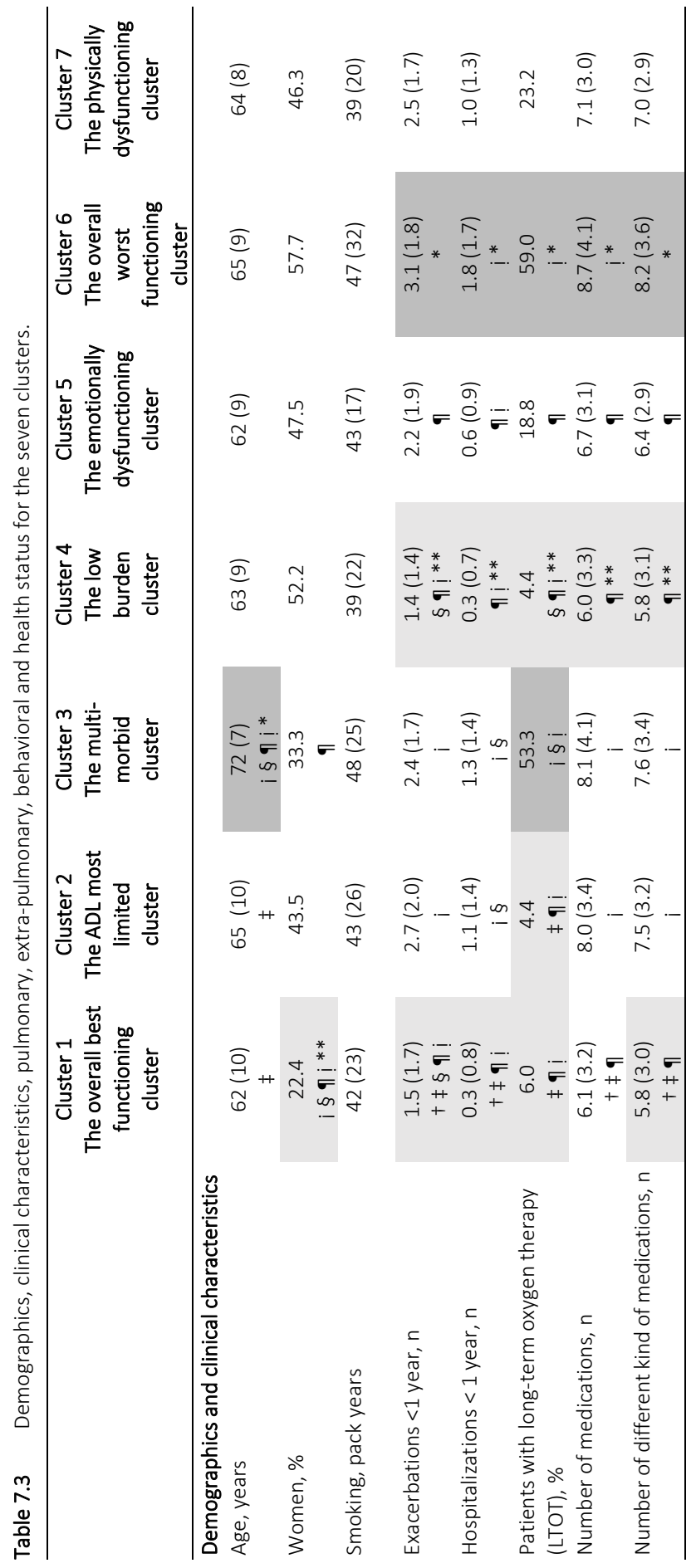




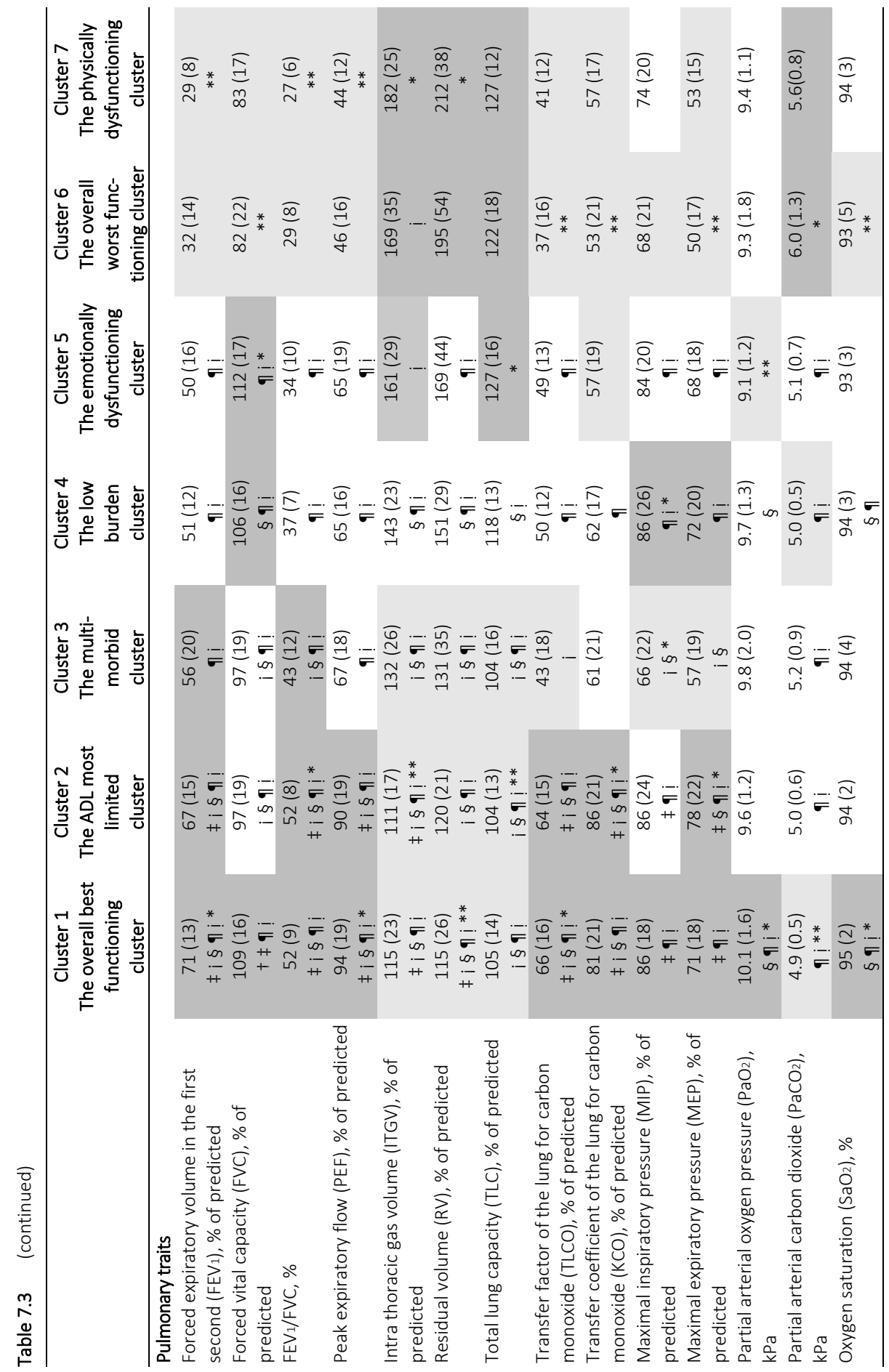




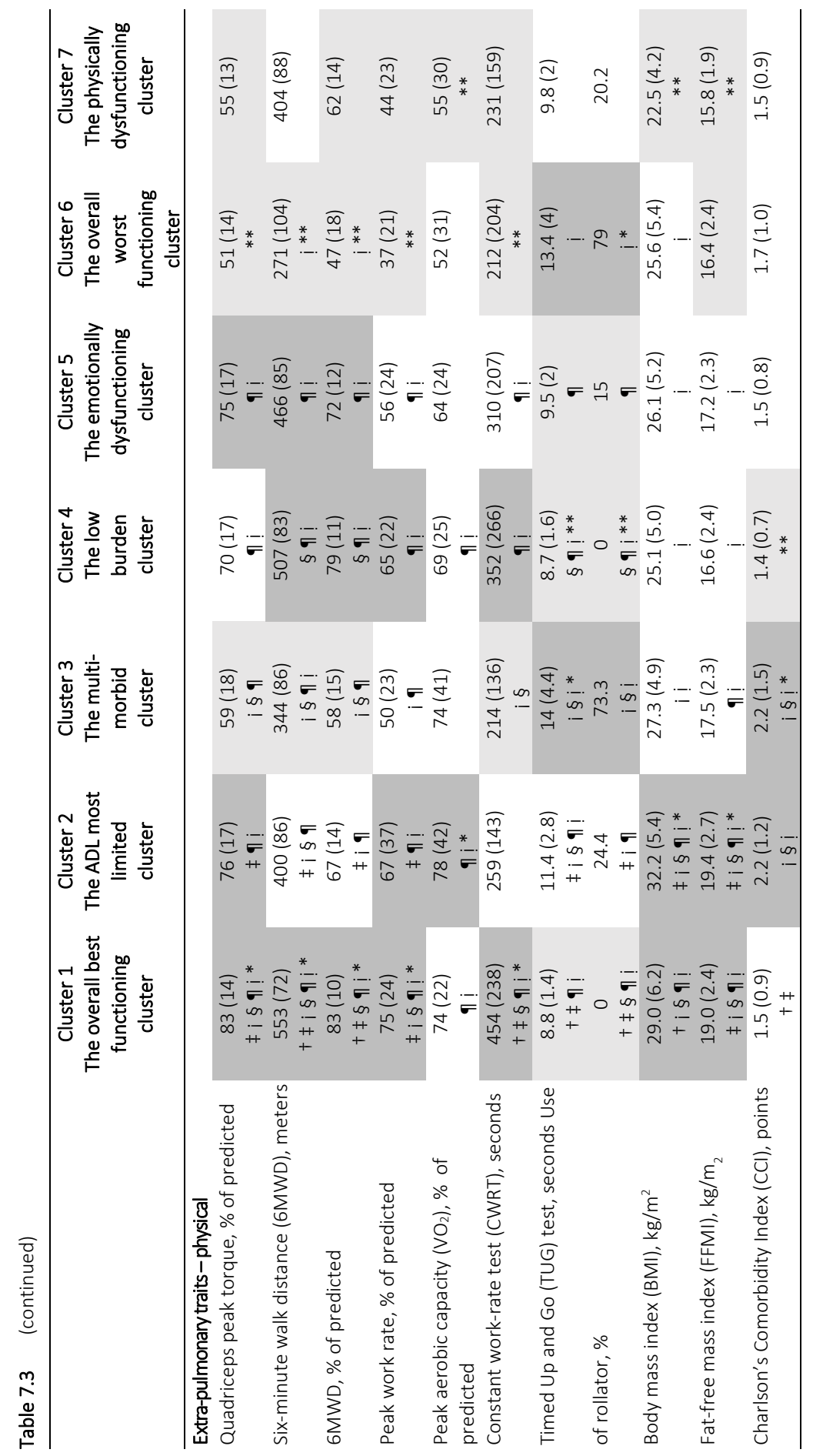




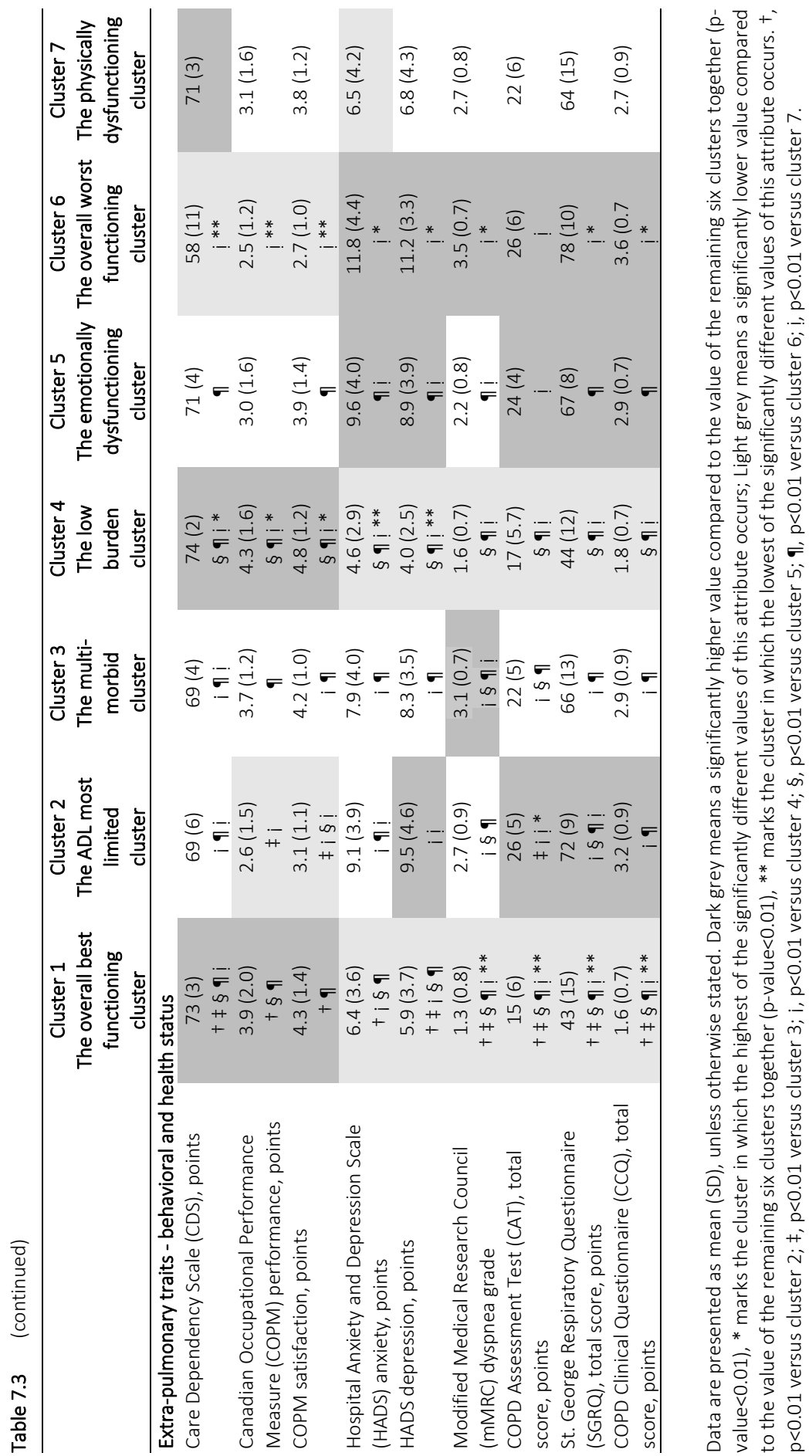




\section{Discussion}

In a sample of patients with moderate to very severe COPD referred for pulmonary rehabilitation, clustering based on a thorough assessment of a broad set of pulmonary and extra-pulmonary (functional, behavioral) traits and health status, resulted in 7 distinct clusters. However, heterogeneity within clusters and overlap regarding individual traits between clusters remain large. Our data suggest that a broad assessment of treatable traits might be useful in individual patients in order to offer personalized management.

Our data clearly illustrate that current recommendations to assess and grade COPD on spirometry, dyspnea and/or health status and exacerbations and/or hospitalizations in the last 12 months largely underestimate the number and heterogeneity of traits involved in the functional and emotional disability as experienced by these frail chronic patients. ${ }^{6}$ To move the field of chronic airway diseases toward precision medicine, a strategy based on the presence and identification of treatable traits was suggested. ${ }^{8}$ The treatable traits approach builds on the concept that by recognizing the clinical complexity of airway diseases such as COPD, more precise and more effective therapies can be offered. ${ }^{8}$ At least, it is assumed that such a treatable traits approach can personalize treatment based on identification of disease characteristics in each individual. ${ }^{34}$ This theoretical framework is never analyzed in a real-life setting of very disabled patients with COPD; neither does it provide evidence on how to tackle the identified traits in different domains. ${ }^{8}$ As early as 1981, pulmonary rehabilitation had been introduced as an art of medical practice wherein an individually tailored, multidisciplinary program was formulated through accurate diagnosis, therapy, emotional support and education to stabilize or reverse both physiopathologic and psychopathologic manifestations of pulmonary diseases. ${ }^{35}$ Nowadays, pulmonary rehabilitation is defined as a comprehensive intervention based on a "thorough" patient assessment followed by patient-tailored therapies designed to improve the physical and psychological condition of patients with chronic respiratory disease and to promote the long-term adherence to health-enhancing behaviors. ${ }^{9}$ The "thorough" assessment as described offers the possibility to check in practice the clinical relevance and practicability of the treatable trait concept.

Previously, we reported the heterogeneity and overlap in identifiable respiratory physiomic clusters and the limited predictability of clustering based on comprehensive lung function assessment for functional performance, health status and outcome of pulmonary rehabilitation. ${ }^{15,36}$ The present study, including extra-pulmonary and behavioral attributes, extends this observation. Despite a comparable respiratory and functional impairment, patients can still vary widely in behavioral traits, resulting in a worse health status (clusters 6 and 7) and even in case of preservation of lung function, significant extra-pulmonary trait involvement can be present (clusters 3 and 6). Referral 

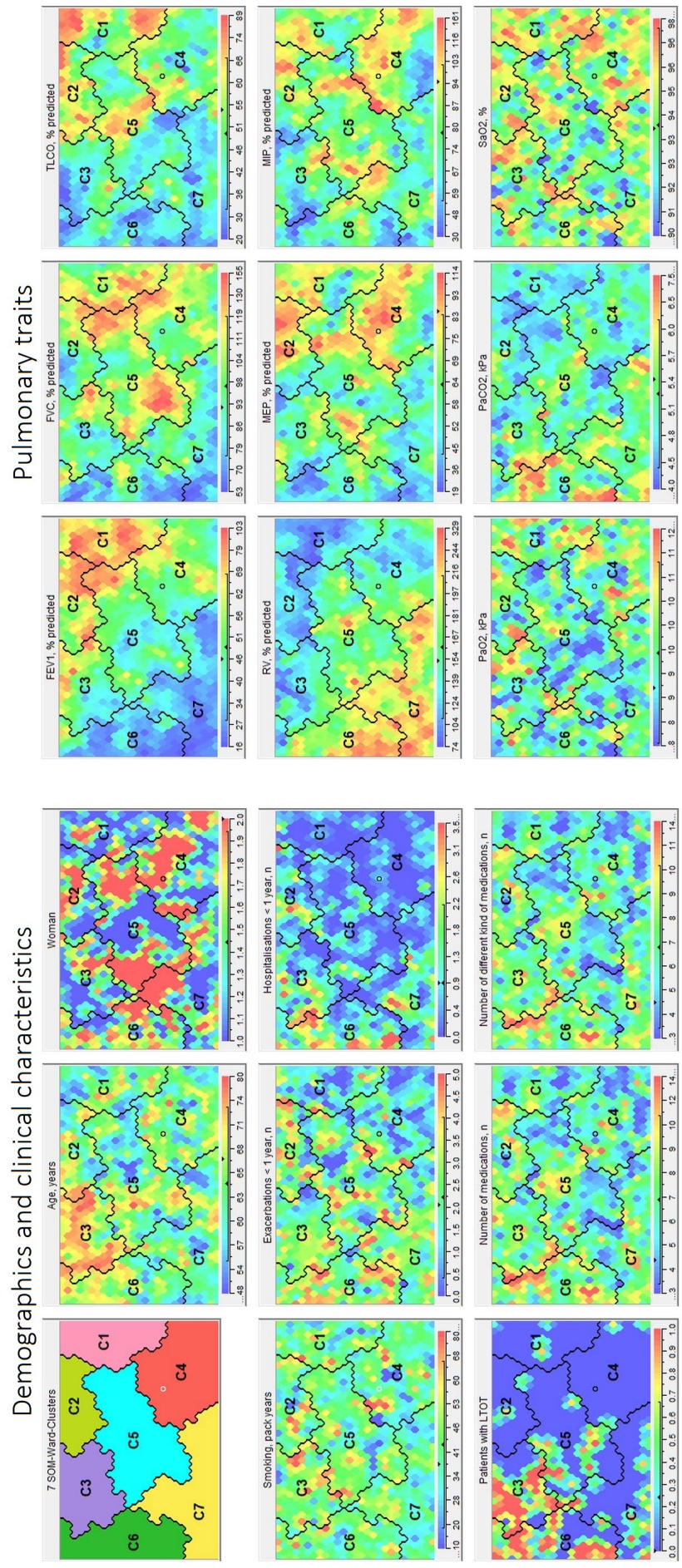


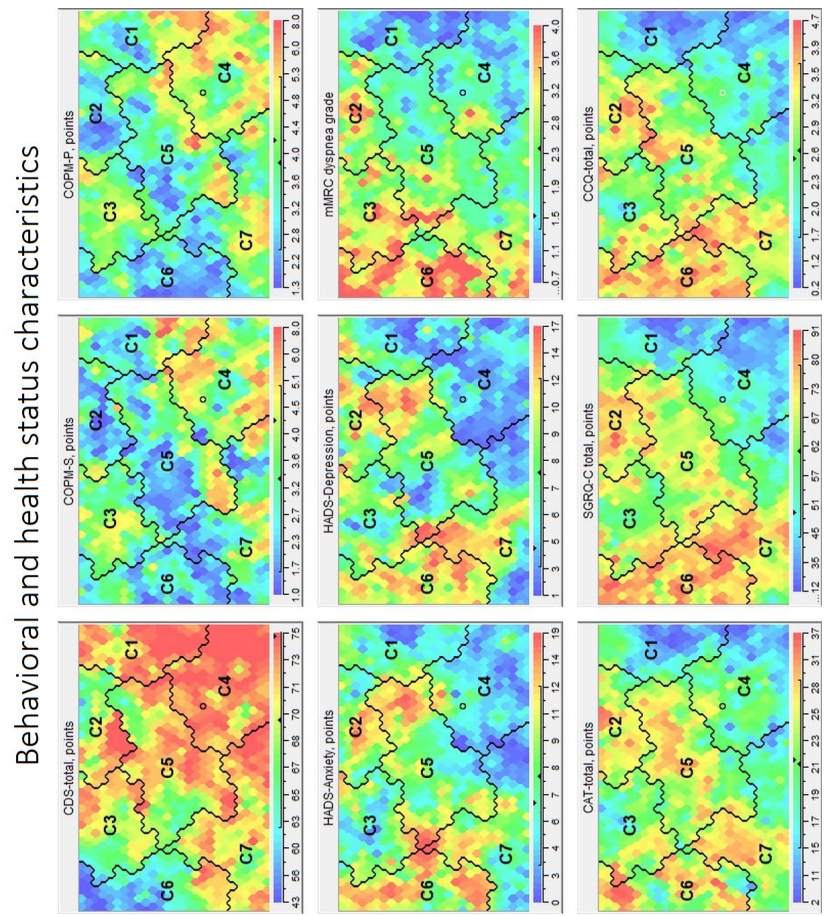

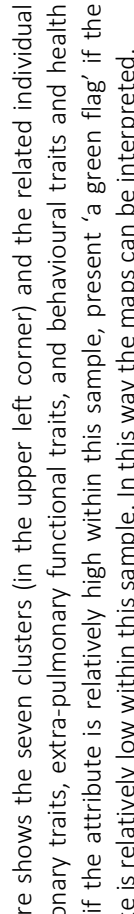

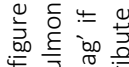
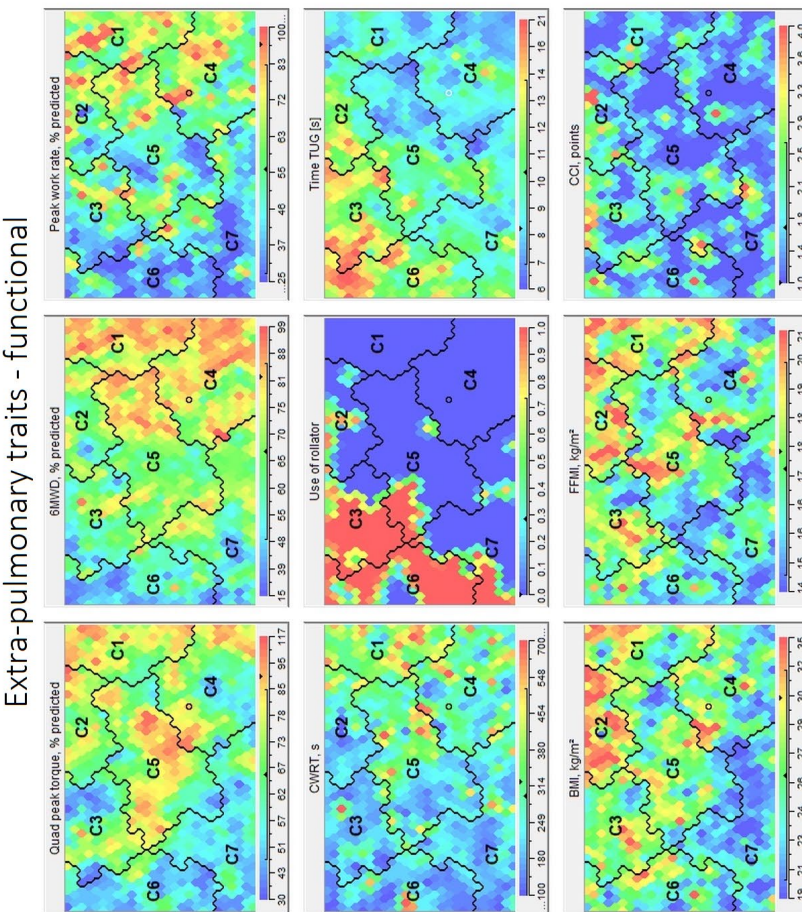

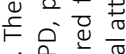

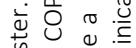

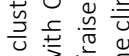

넌

咅离告

으 용 ज

$\lambda \subseteq$ ज它

돈

约

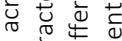

눙

늏 它

可

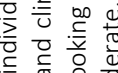

4 뜨을

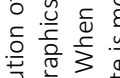

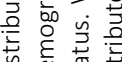

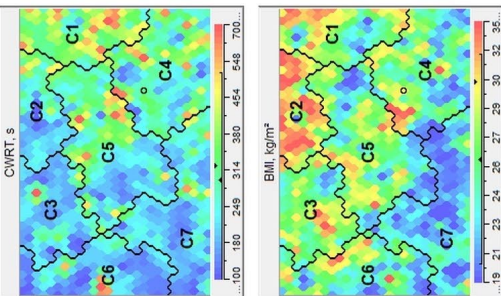

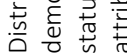

กั 
of patients by chest physicians as in this study does not result in any streamlining of patient cohorts. This is best illustrated by the contrasts in traits between clusters 1 and 6 in our data. We assume that medical management of even disabled patients still relies on symptomatic pharmacologic therapy and the overwhelming focus of assumed risk reduction by prevention of exacerbations. ${ }^{6}$ Intriguingly, all clusters with high anxiety and depression scores (clusters 2, 3, 5, and 6) have a manifested lower health status, confirming the impact of psychologic functioning on health outcomes in COPD. ${ }^{37,38}$ Previous cluster analyses also underscores not only the impact of behavior on experienced health status but also the specificity of these behavioral characteristics for COPD patients. ${ }^{12,39}$

Our data illustrate that a cluster-based management approach is not a feasible option for these patients and that thorough assessment still has to be considered as the start of an individualized intervention. However, pulmonary rehabilitation is more than just problem-oriented care. Previous studies have reported that choosing a limited set of outcomes as key performance indicators ignores the clinical complexity of these rehabilitating patients and that a multidimensional response outcome needs to be considered to study the outcomes of these personalized interventions. The same studies showed the non-linear and differential response after such interventions varying from very good responders to poor responders. ${ }^{11,36}$ Otherwise, the cluster analysis seems very helpful to identify specific targets or topics to improve patient-related outcomes as part of pulmonary rehabilitation. As stated before, our cluster analysis underscores the impact of mood problems in certain COPD clusters. Untreated and under-recognized depression and anxiety symptoms in patients with COPD have effects on physical functioning and on social interaction, increasing fatigue and leading to higher health care utilization. ${ }^{40,41}$ More focused intervention strategies to overcome these mood problems will contribute to better outcomes of such intervention programs. ${ }^{38,42,43}$ At present, psychologists are only involved in one quarter of rehabilitation programs. ${ }^{44}$ Striking is the female predominance in those clusters that have a high disease burden and very severe problems regarding all identified traits. These data can contribute to the consideration of gender-specific issues at least as part of pulmonary rehabilitation. The influence of gender on the expression of COPD is still poorly evaluated. Previous data have reported that women, irrespective of the degree of airflow limitation, had more exacerbations, expressed more dyspnea and had worse scores in all domains of health status suggesting that women may be differentially affected by the disease. Furthermore, women manifested varying exercise capacity and nutritional status, with lower comorbidity scores. ${ }^{45}$ Our data illustrate not only the need to evaluate gender differences in COPD but also to consider gender-specific treatment strategies as well as gender-specific outcomes in personalized treatment strategies. ${ }^{46}$ Finally, our cluster analysis demonstrated that personalized treatment must include the management of the disease beyond the respiratory system. ${ }^{47}$ 
The current study has some limitations. First, the study was conducted in patients with COPD referred by chest physicians for pulmonary rehabilitation. Therefore, our findings are only applicable to this subgroup of highly symptomatic patients and cannot be generalized to the COPD population at large. Second, our study does not include an ageand gender-matched control population, which would have allowed us to better understand the specificity of certain extra-pulmonary and behavioral traits in particular. Third, our findings need to be reproduced in other pulmonary rehabilitation cohorts. Fourth, although the characterization of the patients was very extensive, several traits such as imaging and sleep-related breathing disorders were not included. Fifth, cluster analysis is conducted on cross-sectional data at the start of the rehabilitation program: reproducibility and variability over time is not evaluated. Sixth, the current cluster analysis aims to identify manageable targets in COPD and not underlying pathogenetic pathways. Finally, potential differences in long-term outcomes between clusters were not analyzed. Otherwise, some strengths of our study can also be put forward. First, all included patients underwent a thorough single-center assessment conducted by trained technicians according to state-of-the-art methodology. Second, the density of impairment in the different traits is very high, illustrating that the population is representative for the more complex COPD population.

\section{Conclusion and implications}

Based on respiratory, functional and emotional treatable traits, patients with COPD were divided in 7 clusters: the overall best functioning cluster; the ADL-limited cluster; the multi-morbid cluster; the low burden cluster; the emotionally dysfunctioning cluster; the overall worst-functioning cluster and the physically dysfunctioning cluster. Our data clearly illustrate the tremendous heterogeneity in manifested pulmonary, extrapulmonary functional and behavioral traits in patients with COPD, even persisting after management of the disease by chest physicians. These findings support the need for a thorough assessment to identify an integrated, personalized management in patients suffering from complex conditions as COPD. 


\section{References}

1. Kohansal $\mathrm{R}$, et al. The natural history of chronic airflow obstruction revisited: an analysis of the Framingham offspring cohort. Am J Respir Crit Care Med. 2009;180(1):3-10.

2. Rutten EP, et al. Various Mechanistic Pathways Representing the Aging Process Are Altered in COPD. Chest. 2016;149(1):53-61.

3. Mercado, N, Ito K, Barnes PJ. Accelerated ageing of the lung in COPD: new concepts. Thorax. 2015;70(5): 482-9.

4. Lakhdar R, et al. Role of accelerated aging in limb muscle wasting of patients with COPD. Int J Chron Obstruct Pulmon Dis. 2018;13:1987-98.

5. Rennard SI, Vestbo J. The many "small COPDs": COPD should be an orphan disease. Chest. 2008;134(3): 623-7.

6. GOLD, Global Strategy for the Diagnosis, Management, and Prevention of Chronic Obstructive Pulmonary Disease (2019 report) https://goldcopd.org/wp-content/uploads/2019/12/GOLD-2020-FINALver1.2-03Dec19_WMV.pdf, 2020.

7. Smid DE, et al. Burden of COPD in patients treated in different care settings in the Netherlands. Respir Med. 2016;118:76-83.

8. Agusti A, et al. Treatable traits: toward precision medicine of chronic airway diseases. Eur Respir J. 2016; 47(2):410-9.

9. Spruit MA, et al. An official American Thoracic Society/European Respiratory Society statement: key concepts and advances in pulmonary rehabilitation. Am J Respir Crit Care Med. 2013;188(8):e13-64.

10. Spruit MA, Wouters EFM. Organizational aspects of pulmonary rehabilitation in chronic respiratory diseases. Respirology. 2019.

11. Spruit MA, et al. Differential response to pulmonary rehabilitation in COPD: multidimensional profiling. Eur Respir J. 2015;46(6):1625-35.

12. Vanfleteren LE, et al. Clusters of comorbidities based on validated objective measurements and systemic inflammation in patients with chronic obstructive pulmonary disease. Am J Respir Crit Care Med. 2013; 187(7):728-35.

13. Cho $\mathrm{MH}$, et al. Cluster analysis in severe emphysema subjects using phenotype and genotype data: an exploratory investigation. Respir Res. 2010;11:30.

14. Burgel PR, Paillasseur JL, Roche N. Identification of clinical phenotypes using cluster analyses in COPD patients with multiple comorbidities. Biomed Res Int. 2014;2014:420134.

15. Augustin IML, et al. The respiratory physiome: Clustering based on a comprehensive lung function assessment in patients with COPD. PLoS One. 2018;13(9):e0201593.

16. Smid DE, et al. Impact of cardiovascular comorbidities on COPD Assessment Test (CAT) and its responsiveness to pulmonary rehabilitation in patients with moderate to very severe COPD: protocol of the Chance study. BMJ Open. 2015;5(7):e007536.

17. Fingleton J, Hardy J, Beasley R., Treatable traits of chronic airways disease. Curr Opin Pulm Med. 2018; 24(1):24-31.

18. Borges $O$. Isometric and isokinetic knee extension and flexion torque in men and women aged 20-70. Scand J Rehabil Med. 1989;21(1):45-53.

19. Ribeiro $F$, et al. Test-retest reliability of lower limb isokinetic endurance in COPD: A comparison of angular velocities. Int J Chron Obstruct Pulmon Dis. 2015;10:1163-72.

20. Holland AE, et al. An official European Respiratory Society/American Thoracic Society technical standard: field walking tests in chronic respiratory disease. Eur Respir J. 2014;44(6):1428-46.

21. American Thoracic S and P. American College of Chest. ATS/ACCP Statement on cardiopulmonary exercise testing. Am J Respir Crit Care Med. 2003;167(2):211-77.

22. van 't Hul A, Gosselink R, Kwakkel G. Constant-load cycle endurance performance: test-retest reliability and validity in patients with COPD. J Cardiopulm Rehabil. 2003;23(2):143-50.

23. Schols AM, et al. Nutritional assessment and therapy in COPD: a European Respiratory Society statement. Eur Respir J 2014;44(6):1504-20.

24. Graat-Verboom L, et al. Whole-Body versus Local DXA-Scan for the Diagnosis of Osteoporosis in COPD Patients. J Osteoporos. 2010;2010:640878. 
25. Charlson ME, et al. A new method of classifying prognostic comorbidity in longitudinal studies: development and validation. J Chronic Dis. 1987;40(5):373-83.

26. Dijkstra A, et al. Further psychometric testing of the Dutch Care Dependency Scale on two different patient groups. Int J Nurs Pract. 2002;8(6):305-14.

27. Annegarn J, et al. Problematic activities of daily life are weakly associated with clinical characteristics in COPD. J Am Med Dir Assoc. 2012;13(3):284-90.

28. Mesquita R, et al. Within-day test-retest reliability of the Timed Up \& Go test in patients with advanced chronic organ failure. Arch Phys Med Rehabil. 2013;94(11):2131-8.

29. Zigmond AS, Snaith RP. The hospital anxiety and depression scale. Acta Psychiatr Scand. 1983;67(6): 361-70.

30. Mahler DA, Wells K. Evaluation of clinical methods for rating dyspnea. Chest. 1988;93(3):580-6.

31. Jones PW, et al. Development and first validation of the COPD Assessment Test. Eur Respir J. 2009;34(3): 648-54.

32. Jones PW, Quirk FH, Baveystock CM. The St George's Respiratory Questionnaire. Respir Med. 1991;85 Suppl B:25-31; discussion 33-7.

33. van der Molen $\mathrm{T}$, et al. Development, validity and responsiveness of the Clinical COPD Questionnaire. Health Qual Life Outcomes. 2003;1:13.

34. McDonald VM, et al. Treatable Traits: a new paradigm for 21(st) century management of chronic airway diseases. Eur Respir J. 2019.

35. Hodgkin JE, et al. American Thoracic Society. Medical Section of the American Lung Association. Pulmonary rehabilitation. Am Rev Respir Dis. 1981;124(5): 663-6.

36. Augustin IML, et al. Comprehensive Lung Function Assessment Does not Allow to Infer Response to Pulmonary Rehabilitation in Patients with COPD. J Clin Med. 2018;8(1).

37. Eisner MD, et al. Influence of anxiety on health outcomes in COPD. Thorax. 2010;65(3):229-34.

38. Yohannes AM, Alexopoulos GS. Depression and anxiety in patients with COPD. Eur Respir Rev. 2014; 23(133):345-9.

39. Triest FJJ, et al. Disease-Specific Comorbidity Clusters in COPD and Accelerated Aging. J Clin Med. 2019; 8(4).

40. Doyle $\mathrm{T}$, et al. Association of anxiety and depression with pulmonary-specific symptoms in chronic obstructive pulmonary disease. Int J Psychiatry Med. 2013;45(2):189-202.

41. Mannino DM, Braman S. The epidemiology and economics of chronic obstructive pulmonary disease. Proc Am Thorac Soc. 2007;4(7):502-6.

42. Pumar MI, et al. Anxiety and depression-Important psychological comorbidities of COPD. J Thorac Dis. 2014;6(11):1615-31.

43. Tselebis A, et al. Strategies to improve anxiety and depression in patients with COPD: a mental health perspective. Neuropsychiatr Dis Treat. 2016;12:297-328.

44. Spruit MA, et al. Differences in content and organisational aspects of pulmonary rehabilitation programmes. Eur Respir J. 2014;43(5):1326-37.

45. de Torres JP, et al. Gender and COPD in patients attending a pulmonary clinic. Chest. 2005;128(4):2012-6.

46. Aryal S, Diaz-Guzman E, Mannino DM. COPD and gender differences: an update. Transl Res. 2013; 162(4):208-18

47. Vanfleteren L, et al. Management of chronic obstructive pulmonary disease beyond the lungs. Lancet Respir Med. 2016;4(11):911-24. 



\section{Chapter 8}

\section{Multidimensional outcome assessment of pulmonary rehabilitation in traits-based clusters of COPD patients}

Ingrid ML Augustin, Frits ME Franssen, Sarah Houben-Wilke, Daisy JA Janssen, Swetlana Gaffron, Herman-Jan Pennings, Frank Smeenk, Willem Pieters, Amber Hoogerwerf, Arent-Jan Michels, Frits van Merode, Emiel FM Wouters,

Martijn A Spruit on behalf of the Ciro Pulmonary Rehabilitation Network

Submitted. 


\section{Abstract}

\section{Background}

Clusters of COPD patients have been reported in order to individualize the treatment program. Neither co-morbidity clusters, nor integrated respiratory physiomics clusters contributed to a better prediction of outcomes. Based on a thoroughly assessed set of pulmonary and extrapulmonary traits at the start of a pulmonary rehabilitation (PR) program, we recently described seven clusters of COPD patients. The aims of this study are to confirm multidimensional differential response and to assess the potential of pulmonary and extra-pulmonary traits-based clusters to predict this multidimensional response to PR in COPD patients.

\section{Methods}

Outcomes of a 40-session PR program for COPD patients, referred by a chest physician, were evaluated based on the minimal clinically important difference (MCID) for 6-minute walk distance (6MWD), cycle endurance time, Canadian Occupational Performance Measure performance and satisfaction scores, Hospital Anxiety and Depression Scale anxiety and depression scores, MRC dyspnea grade and St George's Respiratory Questionnaire. The aforementioned response indicators were used to calculate the overall multidimensional response and patients were grouped in very good, good, moderate and poor responders. In the same way, responses to PR were compared based on seven previously identified pulmonary and extra-pulmonary traits-based clusters.

\section{Results}

Of the whole sample, drop out was $19 \%$ and 419 patients (55.4 \% males, age: $64.3 \pm 8.8, \mathrm{FEV}_{1} \%$ of predicted: $48.9 \pm 20$ ) completed the PR program. Very good responders had significantly worse baseline characteristics with a higher burden of disease, a higher proportion of rollator-users, higher body mass index (BMI), more limitations of activities in daily life, emotional dysfunction, higher symptoms of dyspnea and worse quality of life. Of the seven pre-identified clusters, 'the overall best functioning cluster' and 'the low disease burden cluster' both including the best GMWD, the lowest dyspnea score and the overall best health status, demonstrated attenuated outcomes, while in 'the cluster of disabled patients', $76 \%$ of the patients improved health status with at least 2 times MCID. This 'cluster of disabled patients' as well as 'the multi-morbid cluster', 'the emotionally dysfunctioning cluster', 'the overall worst-functioning cluster' and 'the physically dysfunctioning cluster' all demonstrated improvements in performance and satisfaction for occupational activities (more than $65 \%$ of patients improved with $>1 \mathrm{MCID}$ ), emotional functioning (more than $50 \%$ of patients improved with > $1 \mathrm{MCID}$ ) and overall health status (more than $58 \%$ ).

\section{Conclusion}

The current study confirms the differential response to PR based on multidimensional response profiling. Cluster analysis of baseline traits illustrates that non-linear, clinically important differences can be achieved in the most functionally and emotionally impaired clusters and that 'the overall best functional cluster' as well as 'the low disease burden cluster' had an attenuated outcome. 


\section{Introduction}

Pulmonary rehabilitation (PR) as an integrated, personalized intervention to improve the physical and psychological condition of patients with COPD, is based on a thorough assessment in order to identify treatable traits. ${ }^{1}$ This concept of identification of pulmonary and extra-pulmonary treatable traits has been proposed to apply personalized medicine to each individual and to improve outcomes by recognition of the individual needs. ${ }^{2}$ In practice however, PR programs mostly consist of limited components based on a minimum set of identifiable traits. ${ }^{3}$ Furthermore, current evidence for PR is based on changes in exercise performance and health status while the combination of interventions reflected in a set of multidimensional outcomes, is poorly addressed. ${ }^{4}$ A previous study demonstrated that responses in regular outcomes are differential between patients and distinct multidimensional response profiles could be identified. ${ }^{4}$ However, identification of the right patient for the right program as well as prediction of outcomes remains difficult. ${ }^{4}$ Furthermore, different types of exercise-based care require an optimal profiling of patients with COPD. Recently, an expert-opinion model for referral to exercise-based care has been proposed based on disease instability, burden of disease, physical capacity and activity, irrespective of the widely applied degree of airflow limitation. ${ }^{5}$

Clusters of COPD patients have been reported in order to individualize the treatment programs. ${ }^{6,7}$ Neither comorbidity clusters, nor integrated respiratory physiomics clusters contributed to a better prediction of outcomes and to development of cluster based intervention strategies. ${ }^{8,9}$ Based on a thoroughly assessed set of pulmonary and extrapulmonary traits at the start of a PR program, we recently described seven clusters of COPD patients. ${ }^{10}$

The aims of this study are to confirm the differential response to PR based on a previously reported set of response indicators in COPD patients and to assess the potential of previously identified traits-based clusters in order to predict these differential responses for future design of multidimensional and patient-centered interventions.

\section{Materials and methods}

\section{Study design}

The current analysis is based on the data from the Chance Study: an observational, prospective, single-center study about COPD, health status and cardiovascular comorbidities in relation to the outcomes of PR. ${ }^{11}$ This study was approved by the Medical Ethical Committee of the Maastricht University Medical Centre+ (METC 
11-3-070) and is registered at http://www.trialregister.nl (NTR 3416). All patients gave written informed consent. The baseline results have been described previously. ${ }^{10}$

\section{Study sample}

COPD patients referred by chest physicians for a comprehensive PR program at Ciro (Horn, the Netherlands) were eligible to participate (See Supplemental material S8.1). Ciro is a specialized PR center in the southern part of the Netherlands, for patients suffering from complex underlying respiratory diseases. ${ }^{12}$

\section{Interdisciplinary PR program}

Ciro provides a state-of-the-art interdisciplinary PR program ${ }^{13}$ for patients with COPD consisting of 40 sessions. An integrated 2.5-day pre-rehabilitation assessment, assessing physical, emotional and social traits, formed the basis for an individualized PR program. ${ }^{14}$ PR can be inpatient ( 8 weeks, 5 days/week) or outpatient ( 8 weeks, 3 half days/week, followed by 8 weeks 2 half days/week). Patients were allocated for an outpatient or inpatient setting based on an interdisciplinary evaluation after assessment. In general, only care-dependent patients requiring extensive medical supervision were allocated to an inpatient PR program. The outpatient PR program took place under supervision of Ciro in 6 hospitals in the South East of the Netherlands. At the start and during the program, treatment goals were discussed in partnership with each patient. Interventions included physical exercise training, occupational therapy, nutritional counseling, psychosocial counseling, education and exacerbation management. Physical exercise training consists of strengthening exercises, treadmill walking and stationary cycling. Training intensity was monitored and scheduled at moderate-to-high intensity. Moreover, the training intensity increased during the rehabilitation period, based on dyspnea and fatigue symptom scores. All patients underwent general physical exercise for lower and upper extremities, and daily supervised 30-min outdoor walks. Patients, who were too dyspneic to perform endurance/interval/resistance training, received lower-limb high-frequency neuromuscular electrical stimulation. ${ }^{15}$ Each individualized program was followed by an outcome measurement by trained technicians, who were not involved in the exercise training program. Reasons for drop-outs were not systematically scored but were largely related to interfering exacerbations, requiring hospitalization.

\section{Measurements}

As described previously ${ }^{7,10}$, the pre-rehabilitation assessment includes the identification of pulmonary and extra-pulmonary (functional, behavioral and health status) attributes. For a detailed description see Supplemental material (S8.2).

Changes in the degree of dyspnea were measured using the modified Medical Research Council (mMRC) scale (from grade $0=$ no troubles with breathlessness to grade $4=$ too 
breathless to leave the house). ${ }^{16}$ The COPD-specific version of the St George's Respiratory Questionnaire (SGRQ-C), ranging from 0 (optimal) to 100 points (worst) evaluated changes in health status. ${ }^{17}$ Exercise performance was measured by a 6 -min walk test (six-minute walk distance, 6MWD, change in meters, $\mathrm{m})^{18}$ and a constant workrate test (CWRT, change in cycle time expressed in seconds, s) ${ }^{19}$, performed on a stationary bicycle at $75 \%$ of the pre-determined peak work rate (Carefusion, Houten, the Netherlands). The Canadian Occupational Performance Measure (COPM) was used to identify specific problematic activities of daily life. Patients scored how well they were performing the problematic activities of daily life (performance score; COPM-P) and how satisfied they were with this level of performance (satisfaction score; COPM-S) ${ }^{20}$ Scores range between 1 ("not able to do it" or "not at all satisfied", respectively) to 10 points ("able to do it extremely well" or "extremely satisfied"). Symptoms of anxiety and depression were measured by the Hospital Anxiety and Depression Scale (HADS) with a total score ranging from 0 (optimal) to 21 (worst) points. A score of 11 points or higher indicates a severe mood disturbance. ${ }^{21}$ All outcomes were compared with baseline assessment data.

\section{Statistics}

All statistical analyses were performed using Viscovery SOMine 7.3 build 7427 by Viscovery Software GmbH (www.viscovery.net; Vienna, Austria). Self-organizing maps (SOMs, also referred to as Kohonen maps) were used to create an ordered representation of selected attributes. The SOM method can be viewed as a nonparametric regression technique that simplifies complexity by converting multidimensional data spaces into lower dimensional abstractions. A SOM generates a non-linear representation of the data distribution and allows the user to identify homogeneous data groups visually to reveal meaningful relationships. Using the topology of the created SOM model, clusters have been generated by applying the SOMWard Cluster algorithm of Viscovery, a hybrid algorithm that employs the classical hierarchical method of Ward on top of the SOM topology. When creating a SOM, no replacement of missing values is necessary, since only existing values are used to find the best matching position for each patient.

Based on the overall similarity concerning the pre-rehabilitation assessment, seven clusters could be identified (see Figures 8.1 and 8.2). A detailed description of these clusters was previously reported. ${ }^{10}$ The seven clusters were described as: Cluster 1 , 'the overall best functioning cluster'; Cluster 2, 'the ADL most limited cluster'; Cluster 3, 'the multi-morbid cluster'; Cluster 4, 'the low burden cluster'; Cluster 5, 'the emotionally dysfunctioning cluster'; Cluster 6, 'the overall worst functioning' and Cluster 7, 'the physically dysfunctioning cluster'.

The efficacy of the PR program of the whole sample and after traits-based clustering was evaluated based on the minimal clinically important difference $(M C I D)^{4}$ for the following 
eight response indicators: 6MWD (+ $30 \mathrm{~m})$; CWRT (+ $100 \mathrm{~s})$; COPM-P (+ 2 points); COPM$\mathrm{S}$ (+ 2 points); HADS-A (- 1,5 points); HADS-D (- 1,5 points); MRC dyspnea (-1 grade); and SGRQ-Total (- 4 points). The aforementioned response indicators were used to calculate the overall multidimensional response ${ }^{4}$ in which indicators were weighted as follows: 6MWD: 28\%; cycle endurance time CWRT: 20\%; COPM-P: 6.5\%; COPM-S 6.5\%; HADS-A: $8 \%$; HADS-D: $8 \%$; MRC dyspnea: $8 \%$; and SGRQ-Total: $15 \%$, summing up to $100 \%$. The weights are chosen to be the same as used in Spruit et al. ${ }^{4}$, which were based on estimations of importance of each indicator (i.e. indicators that are widely used to evaluate the effectiveness of PR were given more weight) as well as on the percentage of missing values of the respective indicator. The higher the percentage of missing values was, the lesser weight was given.

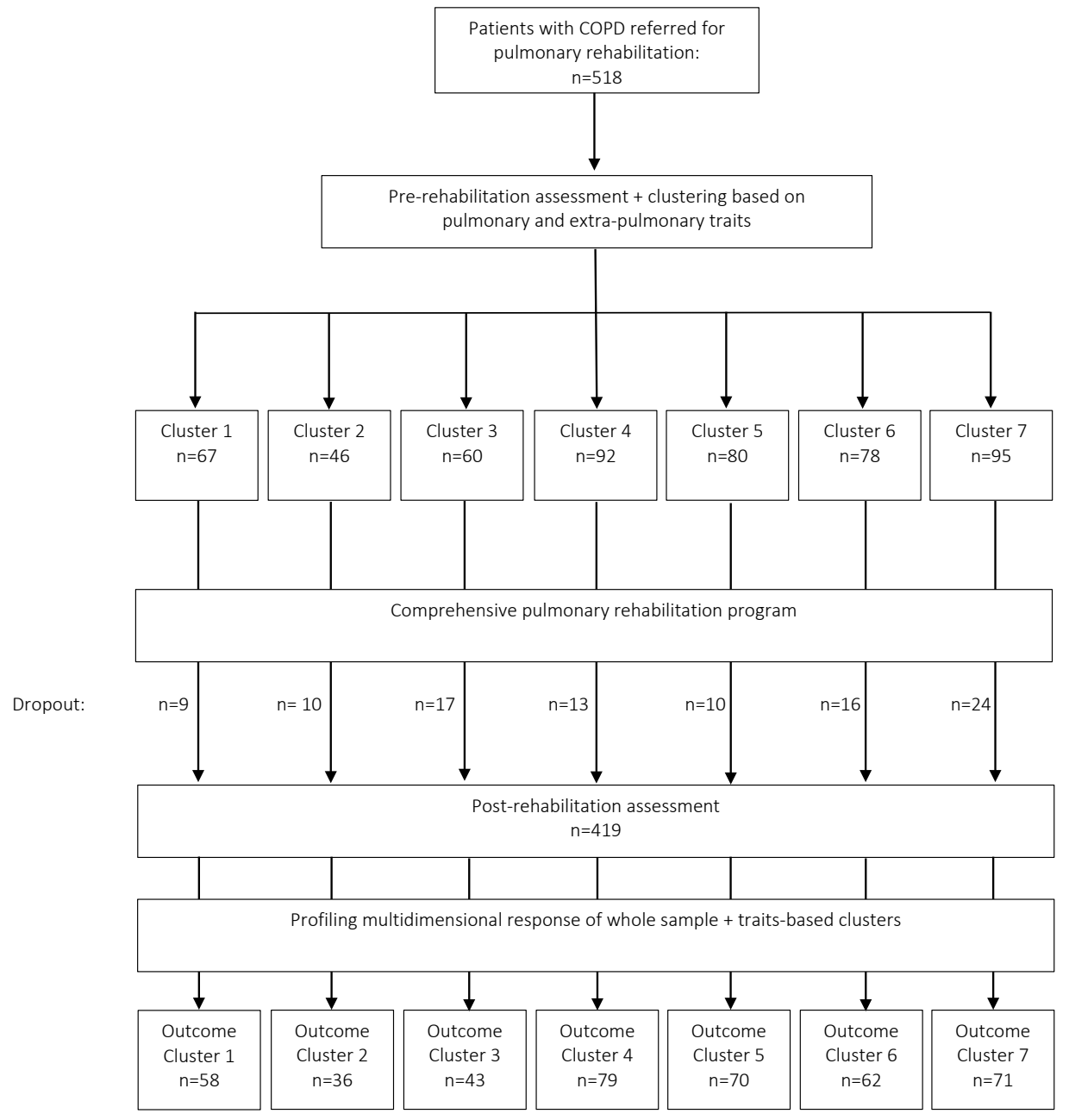

Figure 8.1 Patients before and after comprehensive PR program. 


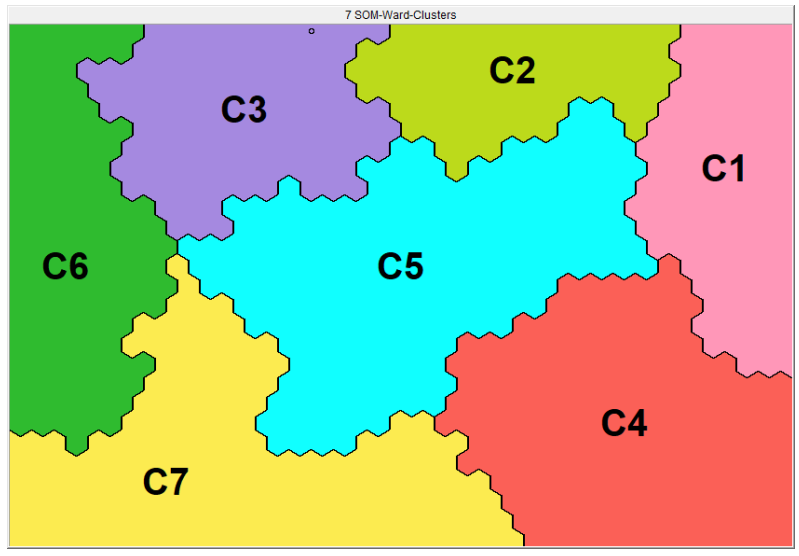

Figure 8.2 Seven different clusters. The Viscovery SOMine program placed all patients with COPD on a specific position of the map based on their baseline characteristics. Subjects located close to each other on the map resemble in terms of their baseline characteristics. Based on the SOM model created, seven SOM-Ward clusters with significantly different profiles have been generated: $\mathrm{C} 1$, 'the overall best functioning cluster'; $\mathrm{C} 2$, 'the ADL-limited cluster'; $\mathrm{C} 3$, 'the multimorbid cluster'; C4, 'the low burden cluster'; C5, 'the emotionally dysfunctioning cluster'; C6, 'the overall worst functioning cluster' and C7, 'the physically dysfunctioning cluster'.

Based on this multidimensional response profiling different groups were generated with substantially different response profiles (very good responders, good responders, moderate responders, and poor responders). Baseline characteristics between these response groups were compared using the integrated two-sided T-test with a confidence level of $99 \%$.

In the same way, responses to PR were compared based on the pre-identified seven clusters based on baseline pulmonary and extra-pulmonary traits.

\section{Results}

\section{Patient characteristics of the whole sample}

518 COPD patients were included (see Table 8.1). They represent COPD patients with mild to very severe airflow limitation, a substantial smoking history, one or more comorbidities, an impaired diffusion capacity and elevated static lung volumes. On average, patients experienced a high impact of the disease on activities in daily life, an impaired health status, had an impaired physical performance and deconditioned quadriceps muscles. $24.1 \%$ of the patients used long term oxygen therapy (LTOT). The mean number of exacerbations as well as hospitalizations in the last year was on average 
2.2 and 0.9 . Accordingly the majority of the patients were therefore classified in GOLD B (26.2\%) and D (54.8\%).

Of the whole sample, drop out was $19 \%$ and 419 patients completed the PR program (see Figure 8.1 and Table 8.1). Of the patients not-completing PR, inspiratory mouth pressure, $6 \mathrm{MWD}$, Timed Up and Go test, anxiety and quality of life were significantly worse compared to the patients completing PR. However, all the other characteristics were comparable between completers and non-completers.

Table 8.1 Demographics, clinical characteristics, pulmonary, extra-pulmonary, behavioral and health status of the whole sample, patients completing and not completing PR.

\begin{tabular}{|c|c|c|c|}
\hline & Whole sample & $\begin{array}{c}\text { Patients completing } \\
\text { pulmonary } \\
\text { rehabilitation }\end{array}$ & $\begin{array}{c}\text { Patients not } \\
\text { completing pulmonary } \\
\text { rehabilitation }\end{array}$ \\
\hline Patients, n (\%) & $518(100)$ & $419(81)$ & $99(19)$ \\
\hline \multicolumn{4}{|l|}{ Demographics and clinical characteristics } \\
\hline Women, \% & 44.4 & 44.6 & 43.4 \\
\hline Age, years & $64.1(9.1)$ & $64.3(8.8)$ & $63.2(10.3)$ \\
\hline Smoking pack years & $42.4(23.6)$ & $42(23.6)$ & $44.1(23.5)$ \\
\hline Exacerbations $<1$ year, $\mathrm{n}$ & $2.2(1.8)$ & $2.2(1.8)$ & $2.3(1.7)$ \\
\hline Hospitalizations $<1$ year, $\mathrm{n}$ & $0.9(1.3)$ & $0.8(1.2)$ & $1.1(1.4)$ \\
\hline Patients with LTOT use, \% & 24.1 & 24.8 & 21.2 \\
\hline Number of respiratory medications, $\mathrm{n}$ & $7.2(3.6)$ & $7.1(3.5)$ & $7.2(3.7)$ \\
\hline $\begin{array}{l}\text { Number of different kind of } \\
\text { medications, } \mathrm{n}\end{array}$ & $6.8(3.2)$ & $6.8(3.2)$ & $6.8(3.4)$ \\
\hline Patients with GOLD I / II /III / IV , \% & 7.3/35.7/36.9/20.1 & $7.6 / 36.3 / 35.3 / 20.8$ & $6.1 / 33.3 / 43.4 / 17.2$ \\
\hline Patients with GOLD A / B / C / D , \% & $10.3 / 26.2 / 8.2 / 54.8$ & 10.6/28.1/8.6/52.0 & $9.1 / 18.2 / 6.1 / 66.3$ \\
\hline \multicolumn{4}{|l|}{ Pulmonary traits } \\
\hline $\mathrm{FEV}_{1}, \%$ predicted & $48.6(20)$ & $48.9(20)$ & $47.3(20.1)$ \\
\hline FVC, \% predicted & $97.5(21.5)$ & $98.5(20.8)$ & $92.9(23.7)$ \\
\hline $\mathrm{FEV}_{1} / \mathrm{FVC}, \%$ & $37.5(12.2)$ & $37.3(12.1)$ & 38.4 (12.9) \\
\hline PEF, \% of predicted & $64.4(24)$ & $64.6(23.7)$ & $63.6(25.2)$ \\
\hline ITGV, \% predicted & 148.7 (35.9) & $148.6(36.3)$ & $148.8(34)$ \\
\hline RV, \% predicted & $161.1(50.7)$ & $160.6(50.9)$ & $163.1(49.7)$ \\
\hline TLC, \% predicted & $117.1(17.5)$ & $117.5(17.3)$ & $115.4(18.2)$ \\
\hline TLCO, \% predicted & $49.3(17.2)$ & $50(17.5)$ & $46.7(15.8)$ \\
\hline $\mathrm{KCO}, \%$ predicted & 64 (21.9) & $64.2(21.9)$ & $62.9(22.1)$ \\
\hline MIP, \% predicted & $78.5(23.3)$ & $79.9(23.6)$ & $72.8(21)^{\#}$ \\
\hline MEP, \% predicted & $63.2(20.4)$ & $64.1(20.6)$ & $59.1(19.2)$ \\
\hline $\mathrm{PaCO}_{2}, \mathrm{kPa}$ & $5.3(0.9)$ & $5.3(0.9)$ & $5.5(0.9)$ \\
\hline $\mathrm{PaO}_{2}, \mathrm{kPa}$ & $9.5(1.5)$ & $9.6(1.5)$ & $9.5(1.6)$ \\
\hline $\mathrm{SaO}_{2}, \%$ & $93.9(3.2)$ & $93.9(3.1)$ & $93.6(3.7)$ \\
\hline \multicolumn{4}{|l|}{ Extra-pulmonary traits - physical } \\
\hline Quadriceps peak torque, \% predicted & $66.2(18.9)$ & $66.7(18.9)$ & $63.9(19)$ \\
\hline Six-MWD, m & $424(124.4)$ & $431.1(123.7)$ & $393(123.3)^{\#}$ \\
\hline Six-MWD, \% predicted & $67.1(18)$ & $68.5(18)$ & $61(16.6)^{\#}$ \\
\hline Peak work rate, \% predicted & $55.5(27.4)$ & $55.9(26.8)$ & $53.9(30)$ \\
\hline Peak $\mathrm{VO}_{2}, \%$ predicted & $66.2(30.4)$ & $66.6(30)$ & $64.2(32.1)$ \\
\hline CWRT, s & $295.5(218.7)$ & $305.1(225)$ & $251.5(181.5)$ \\
\hline TUG, s & $10.5(3.4)$ & $10.3(2.8)$ & $11.6(5)^{\#}$ \\
\hline
\end{tabular}


Table $8.1 \quad$ (continued)

\begin{tabular}{lccc}
\hline & Whole sample & $\begin{array}{c}\text { Patients completing } \\
\text { pulmonary } \\
\text { rehabilitation }\end{array}$ & $\begin{array}{c}\text { Patients not } \\
\text { completing pulmonary } \\
\text { rehabilitation }\end{array}$ \\
\hline Use of rollator, \% & 28.5 & 27.3 & 33.3 \\
BMI, kg/m & $26.2(5.8)$ & $26.2(5.7)$ & $26.2(6.3)$ \\
FFMI, kg/m ${ }^{2}$ & $17.2(2.6)$ & $17.2(2.6)$ & $17.2(2.7)$ \\
CCI, points & $1.6(1)$ & $1.6(0.9)$ & $1.8(1.3)$ \\
\hline Extra-pulmonary traits - behavioral and health status & & $68.4(7.9)$ \\
CDS, points & $69.4(7.3)$ & $69.7(7.2)$ & $3.7(1.3)$ \\
COPM-P, points & $3.9(1.4)$ & $3.9(1.4)$ & $3(1.6)$ \\
COPM-S, points & $3.3(1.7)$ & $3.4(1.7)$ & $9(4.9)^{\#}$ \\
HADS-A, points & $7.8(4.5)$ & $7.5(4.4)$ & $8(4.9)$ \\
HADS-D, points & $7.5(4.3)$ & $7.4(4.2)$ & $2.7(1)$ \\
mMRC dyspnea grade & $2.4(1)$ & $2.4(1)$ & $21.7(6.9)$ \\
CAT, total score, points & $21.5(6.6)$ & $21.5(6.6)$ & $65.4(18.1)^{\#}$ \\
SGRQ, total score, points & $61.1(17.4)$ & $60.1(17.1)$ & $2.8(1.1)$ \\
CCQ, total score, points & $2.6(1)$ & $2.6(1)$ & \\
\hline
\end{tabular}

Data are presented as mean (SD) or as a percentage of the whole sample. BMI, body mass index; CAT, COPD Assessment Test; $\mathrm{CCI}$, Charlson Comorbidity Index; CCQ, Clinical COPD Questionnaire; CDS, Care Dependency Scale; COPM-P, Canadian Occupational Performance Measure-performance with; COPM-S, Canadian

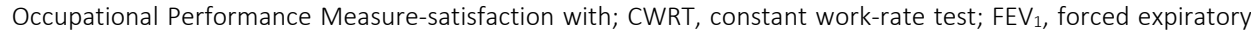
volume in $1 \mathrm{~s}$; FFMI, fat-free mass index; FVC, forced vital capacity; GOLD I, II, III, IV, Global Initiative for Chronic Obstructive Lung Disease classification I (mild = FEV $1 \geq 80 \%$ predicted), II (moderate $=50 \% \leq \mathrm{FEV}_{1}<80 \%$ predicted), III (severe $=30 \% \leq \mathrm{FEV}_{1}<50 \%$ ), IV (very severe $=\mathrm{FEV}^{1}<30 \%$ predicted); GOLD A, B, C, D, Global Initiative for Chronic Obstructive Lung Disease classification A (mMRC 0-1, CAT < 10 and 0 or 1 exacerbation not leading to hospital admission), $B$ ( $M M R C \geq 2, C A T \geq 10$ and 0 or 1 exacerbation not leading to hospital admission), C (mMRC 0-1, CAT $<10$ and $\geq 2$ or $\geq 1$ exacerbation leading to hospital admission), $\mathrm{D}$ ( $\mathrm{mMRC} \geq 2$, CAT $\geq 10$ and $\geq 2$ or $\geq 1$ exacerbation leading to hospital admission); HADS-A, Hospital Anxiety and Depression scale, anxiety scores; HADS-D, Hospital Anxiety and Depression Scale, depression scores; ITGV, intra thoracic gas volume; KCO, the single-breath transfer factor of the lung for carbon monoxide (TLCO) per unit alveolar volume; LTOT, long-term oxygen therapy; MEP, maximal static expiratory mouth pressure; MIP, maximal static inspiratory mouth pressure; $\mathrm{mMRC}$, modified Medical Research Council; $\mathrm{PaCO}_{2}$, arterial partial pressure of carbon dioxide; $\mathrm{PaO}_{2}$, arterial partial pressure of oxygen; $\mathrm{PEF}$, peak expiratory flow in $1 \mathrm{~s}$; RV, residual volume; $\mathrm{SaO}_{2}$, arterial oxygen saturation; SGRQ, St. George's Respiratory Questionnaire; Six-MWD, 6-minute walk distance; TLC, total lung capacity; TUG, Timed Up and Go test; $V_{2}$ : oxygen uptake. \#, $p<0.01$ versus patients completing pulmonary rehabilitation

\section{Multidimensional response profiling of patients completing PR}

Table 8.2 summarizes the improvements following PR for the total group and after stratification for response. On average, improvements were found for 6MWD: $23 \pm 67$ m; CWRT: $206 \pm 306$ s; COPM-P: $2.8 \pm 1.8$ points; COPM-S: $3.5 \pm 2.2$ points; HADS-A: $1.7 \pm 3.7$ points; HADS-D: $2.1 \pm 3.7$ points; MRC: $0.3 \pm 1.1$ and SGRQ total score: $9 \pm 14$ points. A clinically important gain was achieved in $56 \pm 28 \%$ of all outcomes. As expected, the very good responders group included the highest proportion of clinically relevant improvements: $82 \pm 15 \%$ of outcomes exceeding more than 1 MCID and $63 \pm 18 \%$ outcomes exceeding more than 2 MCID. Good responders showed $65 \pm 16 \%$ outcomes exceeding more than $1 \mathrm{MCID}$ and $37 \pm 18 \%$ outcomes exceeding more than 2 MCID. While clinically relevant improvements were significantly lower in moderate and 
poor responders, as an example, 50\% of the patients still improved more than $1 \mathrm{MCID}$ for COPM-P and $60 \%$ for COPM-S in the group of moderate responders.

Table 8.2 Responses to pulmonary rehabilitation of all patients completing pulmonary rehabilitation.

\begin{tabular}{|c|c|c|c|c|c|}
\hline & $\begin{array}{c}\text { Patients } \\
\text { completing } \\
\text { PR }\end{array}$ & $\begin{array}{l}\text { Very good } \\
\text { responder }\end{array}$ & $\begin{array}{c}\text { Good } \\
\text { responder }\end{array}$ & $\begin{array}{l}\text { Moderate } \\
\text { responder }\end{array}$ & $\begin{array}{c}\text { Poor } \\
\text { responder }\end{array}$ \\
\hline $\begin{array}{l}\text { Patients, } \mathrm{n} \text { (\% patients } \\
\text { completing PR) }\end{array}$ & $419(100)$ & $108(26)$ & $146(35)$ & $123(29)$ & $42(10)$ \\
\hline$\Delta 6 \mathrm{MWD}, \mathrm{m}$ & $22.9(67)$ & $86.7(66.2)$ & $26.7(31.9) \#$ & $-9(46)^{\# 9}$ & $-65.9(52.7)^{\# 9+}$ \\
\hline$\geq 30 \mathrm{~m}, \%$ patients & 43.7 & 87.5 & $46.5^{\#}$ & $16 \#$ & $0^{\# q+}$ \\
\hline$\geq 60 \mathrm{~m}, \%$ patients & 21.8 & 62.5 & $14.8^{\#}$ & $1.7^{\# \oplus}$ & $0^{\#}$ \\
\hline$\Delta C W R T, s$ & $206.4(306)$ & $442.8(325)$ & $237.7(267)^{\#}$ & $61.9(171.3)^{\# q}$ & $-96.1(197.8)^{\#-+}$ \\
\hline$\geq 100 \mathrm{~s}, \%$ patients & 51.9 & 82.5 & $60.6^{\#}$ & $31.6^{\#-9}$ & $0^{\# q+}$ \\
\hline$\geq 200 \mathrm{~s}, \%$ patients & 36.2 & 75.3 & $37.9^{\#}$ & $12.3^{\# \text { ๆ }}$ & $0^{\# \uparrow}$ \\
\hline$\Delta$ COPM-P, points & $2.8(1.8)$ & $4(1.7)$ & $3.2(1.6)^{\#}$ & $1.8(1.3)^{\# \oplus 9}$ & $0.7(1.3)^{\#-9+}$ \\
\hline$\geq 2$ points, $\%$ patients & 68.3 & 86.5 & 80.6 & $50.5^{\#, 9}$ & $22.9^{\# 9+}$ \\
\hline$\geq 4$ points, $\%$ patients & 26.2 & 54.8 & $27.6^{\#}$ & $5.5^{\text {\#ף }}$ & $0^{\# \oplus}$ \\
\hline$\Delta$ COPM-S, points & $3.5(2.2)$ & $4.7(1.9)$ & $4(1.8)^{\#}$ & $2.5(1.9)^{\# \uparrow}$ & $0.8(1.5)^{\# \text { q++ }}$ \\
\hline$\geq 2$ points, $\%$ patients & 76.6 & 93.3 & 90.3 & $59.6^{\# \oplus 9}$ & $26.5^{\# \cdot+}$ \\
\hline$\geq 4$ points, $\%$ patients & 43.3 & 63.5 & 51.5 & $26.6^{\# \oplus 9}$ & $2.9^{\# \oplus++}$ \\
\hline$\Delta$ HADS-A, points & $1.7(3.7)$ & $3.7(3.7)$ & $2.2(3.2)^{\#}$ & 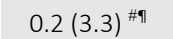 & $-0.9(2.9)$ \\
\hline$\geq 1.5$ points, $\%$ patients & 50.5 & 71.9 & $54.9^{\#}$ & $36.5^{\# \bullet}$ & $19.4^{\# \text { \# }}$ \\
\hline$\geq 3.0$ points or more, $\%$ pts & 39.3 & 62.5 & $39.9^{\#}$ & $27.1^{\#}$ & $11.1^{\# \text { ๓ }}$ \\
\hline$\Delta$ HADS-D, points & $2.1(3.7)$ & $3.9(3.5)$ & $3(3.5)$ & $0.5(3.1)^{\# \uparrow}$ & $-0.7(3)^{\# \uparrow}$ \\
\hline$\geq 1.5$ points, $\%$ patients & 53 & 69.8 & 62.4 & $37^{\# \text { \# }}$ & $19 \#$ \\
\hline$\geq 3.0$ points, $\%$ patients & 38.7 & 57.3 & 47.4 & $23.4^{\# \oplus 9}$ & $2.8^{\# 9++}$ \\
\hline$\Delta \mathrm{mMRC}$ dyspnea, grade & $0.3(1.1)$ & $1(1.1)$ & $0.3(1)^{\#}$ & $-0.1(1)^{\#}$ & $-0.2(0.8)^{\#}$ \\
\hline$\geq 1$ grade, $\%$ patients & 38.9 & 67.6 & $38.7^{\#}$ & $23^{\#}$ & $13.8^{\#}$ \\
\hline$\geq 2$ grades, $\%$ patients & 15.6 & 33.8 & $14^{\#}$ & $6.9^{\#}$ & $0^{\#}$ \\
\hline$\triangle S G R Q$ total score, points & $9.1(14)$ & $19.2(11.7)$ & $12.2(12.7)^{\#}$ & $1.7(10.5)^{\# \oplus 9}$ & $-4.9(9.3)^{\#-+}$ \\
\hline$\geq 4$ points, $\%$ patients & 61.6 & 89.7 & $76.3^{\#}$ & $36 \#$ & $15.4^{\# \text { ॥ }}$ \\
\hline$\geq 8$ points or more, $\%$ pts & 50.9 & 83.5 & $63.7^{\#}$ & $24.6^{\# \oplus 9}$ & $2.6^{\# \uparrow++}$ \\
\hline Outcomes exceeding $\geq 1 \mathrm{MCID}, \%$ & $55.8(27.8)$ & $82(15.5)$ & $65(16.1)^{\#}$ & $36.4(18.7)^{\# ף}$ & $13.4(15.1)^{\# \cdot++}$ \\
\hline Outcomes exceeding $\geq 2 \mathrm{MCID}, \%$ & $34.2(25.9)$ & $62.7(18.1)$ & $37.5(18.1)^{\#}$ & $16.2(14.1)^{\# \uparrow}$ & $2.3(6.1)^{\# q_{+}}$ \\
\hline
\end{tabular}

See legend Table 8.1 for explanation of abbreviations. Data are presented as mean (SD), unless otherwise stated. $\Delta$, improvement (a minus sign means a deterioration); a lower score for HADS, mMRC dyspnea and SGRQ is an improvement. Outcomes exceeding $\geq x \mathrm{MCID}, \%$ : Percentage of outcomes which exceed the pre-defined minimal clinically important difference (MCID) at least $x$ times. ${ }^{\#}, p<0.01$ versus very good responder cluster; ${ }^{\natural}, p<0,01$ versus good responder cluster; ${ }^{+}, p<0.01$ versus moderate responder cluster. When the value is significantly higher versus all the other clusters, the table-cell is colored dark grey; if it is significantly lower, it is colored light grey.

\section{Baseline characteristics after stratification for multidimensional response clusters}

Table 8.3 summarizes the baseline characteristics of the very good responders, good, moderate and poor responders. Compared to the other groups, very good responders had significantly worse characteristics with a higher burden of disease, a higher proportion of rollator-users, higher BMI, higher limitations of activities in daily life, emotional dysfunction, higher symptoms of dyspnea and worse quality of life, while moderate responders demonstrated less hospitalizations, less limitations of activities in daily life, lower symptoms of dyspnea and a higher quality of life. Very good responders 
showed significantly better pulmonary traits compared to the other groups. The proportion of patients following an inpatient program was significantly higher in the very good responders compared to good, moderate and poor responders.

Table 8.3 Demographics, clinical characteristics, pulmonary, extra-pulmonary, behavioral and health status of very good, good, moderate and poor responders.

\begin{tabular}{|c|c|c|c|c|}
\hline & $\begin{array}{l}\text { Very good } \\
\text { responder }\end{array}$ & $\begin{array}{c}\text { Good } \\
\text { responder }\end{array}$ & $\begin{array}{l}\text { Moderate } \\
\text { responder }\end{array}$ & $\begin{array}{c}\text { Poor } \\
\text { responder }\end{array}$ \\
\hline $\begin{array}{l}\text { Patients, } \mathrm{n} \text { (\% patients } \\
\text { completing PR) }\end{array}$ & $108(26)$ & $146(35)$ & $123(29)$ & $42(10)$ \\
\hline \multicolumn{5}{|c|}{ Demographics and clinical characteristics } \\
\hline Women, \% & 43.5 & 46.6 & 43.1 & 45.2 \\
\hline Age, years & $63.6(8.8)$ & $63.8(9.2)$ & $64.8(8.3)$ & $66.7(8.9)$ \\
\hline Smoking pack years & $42.7(21.1)$ & $40.2(18.9)$ & $44.4(30.4)$ & $39.4(21.1)$ \\
\hline Exacerbations $<1$ year, $\mathrm{n}$ & $2.9(1.9)$ & $2.1(1.9)^{\#}$ & $1.9(1.6)^{\#}$ & $1.9(1.6)^{\#}$ \\
\hline Hospitalizations $<1$ year, $\mathrm{n}$ & $1(1.2)$ & $0.9(1.4)$ & $0.6(1)^{\#}$ & $1(1.3)$ \\
\hline Patients with LTOT use, \% & 27.8 & 25.3 & 23.6 & 19.1 \\
\hline $\begin{array}{l}\text { Number of respiratory } \\
\text { medications, } \mathrm{n}\end{array}$ & $8(3.6)$ & $7.1(3.8)$ & $6.6(3.2)$ \# & $6.5(3)$ \\
\hline $\begin{array}{l}\text { Number of different kind of } \\
\text { medications, } n\end{array}$ & $7.7(3.4)$ & $6.7(3.3)$ & $6.4(3)^{\#}$ & $6.3(2.9)$ \\
\hline Patients with GOLD I/II/III/IV, \% & 9.3/39.8/33.3/17.6 & $6.9 / 35.6 / 32.9 / 24.7$ & $7.3 / 32.5 / 39 / 21.1$ & $7.1 / 40.5 / 38.1 / 14.3$ \\
\hline Patients with GOLD A/B/C/D, \% & $4.6 / 21.3 / 9.3 / 64.8$ & $10.4 / 35.4 / 6.2 / 46.9^{\#}$ & ${ }^{\#} 16^{\#} / 25 / 10 / 48{ }^{\#}$ & $9.5 / 28.6 / 12.2 / 48.8$ \\
\hline \multicolumn{5}{|l|}{ Pulmonary traits } \\
\hline $\mathrm{FEV}_{1}, \%$ predicted & $50.6(19.9)$ & $47.6(20.2)$ & $48.2(19.4)$ & $50.7(21.4)$ \\
\hline FVC, $\%$ predicted & $94.8(21.2)$ & $99.6(21.5)$ & $100.3(19.3)$ & $99.3(21.2)$ \\
\hline $\mathrm{FEV}_{1} / \mathrm{FVC}, \%$ & $40.3(12.8)$ & $35.8(11.5)^{\#}$ & $36.1(11.5)^{\#}$ & $38.2(12.7)$ \\
\hline PEF, \% of predicted & $69.6(24.9)$ & $63.7(22.8)$ & $61.4(22.5)^{\#}$ & $64(26)$ \\
\hline ITGV, \% predicted & $139.6(37.8)$ & $151(32.8)$ & $152.2(35.6)$ & $150.7(44)$ \\
\hline $\mathrm{RV}, \%$ predicted & $152.1(52.9)$ & $165.5(45.3)$ & $161.7(52)$ & $160.4(60.8)$ \\
\hline TLC, \% predicted & $113.5(18.4)$ & $119.3(16.2)^{\#}$ & $118.6(16.2)$ & $117.8(20.6)$ \\
\hline TLCO, \% predicted & $54.7(17.2)$ & $49(18)$ & $47.3(16)^{\#}$ & $49.8(19)$ \\
\hline $\mathrm{KCO}, \%$ predicted & $72.2(24.3)$ & $61.3(19.7)^{\#}$ & $61.4(20.3)^{\#}$ & $64(23.7)$ \\
\hline MIP, \% predicted & $81.5(20.5)$ & $79.4(25)$ & $81.2(23.5)$ & $73.3(26.1)$ \\
\hline MEP, \% predicted & $64.9(20.8)$ & $65.6(21.5)$ & $62.9(19)$ & $61(21.5)$ \\
\hline $\mathrm{PaCO}_{2}, \mathrm{kPa}$ & $5.4(1)$ & $5.3(0.9)$ & $5.2(0.8)$ & $5.2(0.8)$ \\
\hline $\mathrm{PaO}_{2}, \mathrm{kPa}$ & $9.6(1.7)$ & $9.6(1.4)$ & $9.5(1.4)$ & $9.4(1.5)$ \\
\hline $\mathrm{SaO}_{2}, \%$ & $93.8(3.7)$ & $94.1(2.6)$ & $94(2.9)$ & $93.6(3.3)$ \\
\hline \multicolumn{5}{|l|}{ Extra-pulmonary traits - physical } \\
\hline $\begin{array}{l}\text { Quadriceps peak torque, \% } \\
\text { predicted }\end{array}$ & $69.4(18.9)$ & $65.3(17.6)$ & $67(18.8)$ & $64.4(23)$ \\
\hline $6 M W D, m$ & $404.8(140.2)$ & $432(121.1)$ & $455.9(104.8)^{\#}$ & $422.9(128.5)$ \\
\hline $6 \mathrm{MWD}, \%$ predicted & $66.3(19.8)$ & $68.2(18.1)$ & $70.9(15.4)$ & $67.7(19.5)$ \\
\hline Peak work rate, \% predicted & $55.7(27.1)$ & $54.9(27.6)$ & $57.5(27)$ & $54.8(22.6)$ \\
\hline Peak $\mathrm{VO}_{2}, \%$ predicted & $70.2(30.7)$ & $65.2(30.9)$ & $65.1(28.1)$ & $67.5(31.1)$ \\
\hline CWRT, s & $318.4(222.6)$ & $286.8(216.4)$ & $304.8(215.5)$ & $335.8(283.7)$ \\
\hline TUG, s & $10.8(3.1)$ & $10.4(3)$ & $9.7(2.2)^{\#}$ & $10.4(2.9)$ \\
\hline Use of rollator, $\%$ & 37.4 & 28.1 & $19.7^{\#}$ & 21.4 \\
\hline $\mathrm{BMI}, \mathrm{kg} / \mathrm{m}^{2}$ & $28.7(6.3)$ & $26.3(5.5)^{\#}$ & $24.2(4.8) \#$ & $25.7(4.8)^{\#}$ \\
\hline $\mathrm{FFMI}, \mathrm{kg} / \mathrm{m}^{2}$ & $18.2(2.9)$ & $16.9(2.4)^{\#}$ & $16.7(2.3)^{\#}$ & $16.8(2.5)^{\#}$ \\
\hline $\mathrm{CCl}$, points & $1.7(1)$ & $1.5(0.8)$ & $1.6(0.9)$ & $1.9(1.2)$ \\
\hline
\end{tabular}


Table 8.3 (continued)

\begin{tabular}{|c|c|c|c|c|}
\hline \multicolumn{5}{|c|}{ Extra-pulmonary traits - behavioral and health status } \\
\hline CDS, points & $69(7.6)$ & $69.3(6.8)$ & $70.8(5.9)$ & $69.3(10.3)$ \\
\hline COPM-P, points & $3.5(1.2)$ & $3.8(1.4)$ & $4.2(1.3)^{\#}$ & $4.5(1.6)^{\#}$ \\
\hline COPM-S, points & $2.9(1.6)$ & $3.2(1.6)$ & $3.8(1.7)^{\# \oplus 9}$ & $4.1(1.8)^{\# \oplus \pi}$ \\
\hline HADS-A, points & $8.7(4.4)$ & $7.9(4.3)$ & $6.4(4.2)^{\# \oplus}$ & $6.8(4.1)$ \\
\hline HADS-D, points & $8.7(4.1)$ & $8(4.1)$ & $6.1(4)^{\# \uparrow}$ & $6.2(3.6)^{\#}$ \\
\hline mMRC dyspnea grade & $2.6(1.1)$ & $2.4(1)$ & $2.1(1)^{\# \uparrow}$ & $2.3(1.1)$ \\
\hline CAT, total score, points & $23.7(6.3)$ & $22.6(5.9)$ & $19(6.3)^{\# \bullet}$ & $19.5(7.4)^{\# \oplus}$ \\
\hline SGRQ, total score, points & $67.5(15)$ & $61(16.1)^{\#}$ & $53.8(16.6) \#$ & $56.9(19.6)^{\#}$ \\
\hline$C C Q$, total score, points & $2.9(1)$ & $2.8(1)$ & $2.2(0.8)$ & $2.4(1.1)$ \\
\hline Inpatient \% & 76 & $60^{\#}$ & $41^{\# \oplus}$ & $38^{\#}$ \\
\hline
\end{tabular}

See legend Table 8.1 for explanation of abbreviations. ", $p<0.01$ versus very good responder cluster; ${ }^{\uparrow}, p<0,01$ versus good responder cluster; ${ }^{+}, \mathrm{p}<0.01$ versus moderate responder cluster. When the value is significantly higher versus all the other clusters, the table-cell is colored dark grey; if it is significantly lower, it is colored light grey.

\section{Responses to PR after traits-based clustering}

The responses to PR for the seven traits-based clusters are summarized in Table 8.4.

In Cluster 1, 'the overall best functioning cluster' at baseline, drop-out was $13 \%$ with 58 patients completing the PR program. The proportion of patients following an inpatient program was $18 \%$. Except for constant work rate test, which improved significantly after treatment, response indicators in cluster 1 were comparable to the average of the response indicators of the whole sample. Breathlessness even worsened significantly after PR in this cluster.

Despite the higher impairment in $A D L$, higher depression scores and worse quality of life in cluster 2, this 'ADL limited cluster' (drop out 22\%, 36 patients of which 70\% inpatients) manifested a significantly higher proportion of very good responders compared to the other clusters. In addition, the percentage of patients having outcomes exceeding $\geq 2$ MCID was similar to cluster 6 and significantly higher than the other clusters. A significant better response to PR was found for 6 MWD, performance of problematic activities of daily life, symptoms of dyspnea and health status.

Cluster 3, 'the more multi-morbid cluster' (drop out 28\%, 43 patients, 75\% inpatients) with significantly lower values for exercise performance and higher dyspnea scores at baseline showed a response pattern comparable to the whole sample with exception for dyspnea which improved significantly better in this cluster.

Cluster 4 (drop out 14\%, 79 patients, 20\% inpatients), identified as the low burden cluster' had similar baseline characteristics as cluster 1 , but demonstrated the lowest proportion of very good responders, the highest proportion of poor responders, lowest percentage of outcomes exceeding $\geq 1 \mathrm{MCID}$ and lowest percentage of outcomes exceeding $\geq 2$ MCID. Only improvement for CWRT was similar to the entire group. All other indicators responded worse compared to the other clusters. 
Cluster 5, 'the emotionally dysfunctioning cluster' (drop out 13\%, 70 patients, 66\% inpatients) demonstrated at baseline a significantly better 6MWD, but these patients had higher scores for anxiety and depression and worse health status compared to all patients. Although the majority of the response indicators in cluster 5 were comparable to the whole group, PR particularly resulted in an improvement of the burden of depression.

Cluster 6 (drop out 21\%, 62 patients, 92\% inpatients) was identified as the worst functioning cluster'. However, PR resulted in a higher proportion of outcomes with a clinical important difference as well as a higher percentage of very good responders. In particular, a better response was found for 6 MWD, performance of problematic activities of daily life, symptoms of depression and symptoms of dyspnea.

Cluster 7, 'the physically dysfunctioning cluster', (drop out 25\%, 71 patients, 70\% inpatients), showed smallest improvement in physical functioning parameters after PR while other response indicators were comparable to the mean of the whole sample.

The overall response as well as the outcomes, expressed in absolute terms as well as in changes of MCIDs, is illustrated in Figure 8.3. Figure 8.3 clearly illustrates the differential response after PR in COPD, but also that poor responders form only a minority when multidimensional response profiling is conducted and that the different response profiles are distributed over all clusters. The individual components contributing to the multidimensional profile are depicted in Figure 8.3.b. To note is the distribution of the outcomes in performance and satisfaction in performing activities of daily life and the improvement in psychological burden as well as the improvements in experienced health status over the identified clusters at the end of the PR program. 


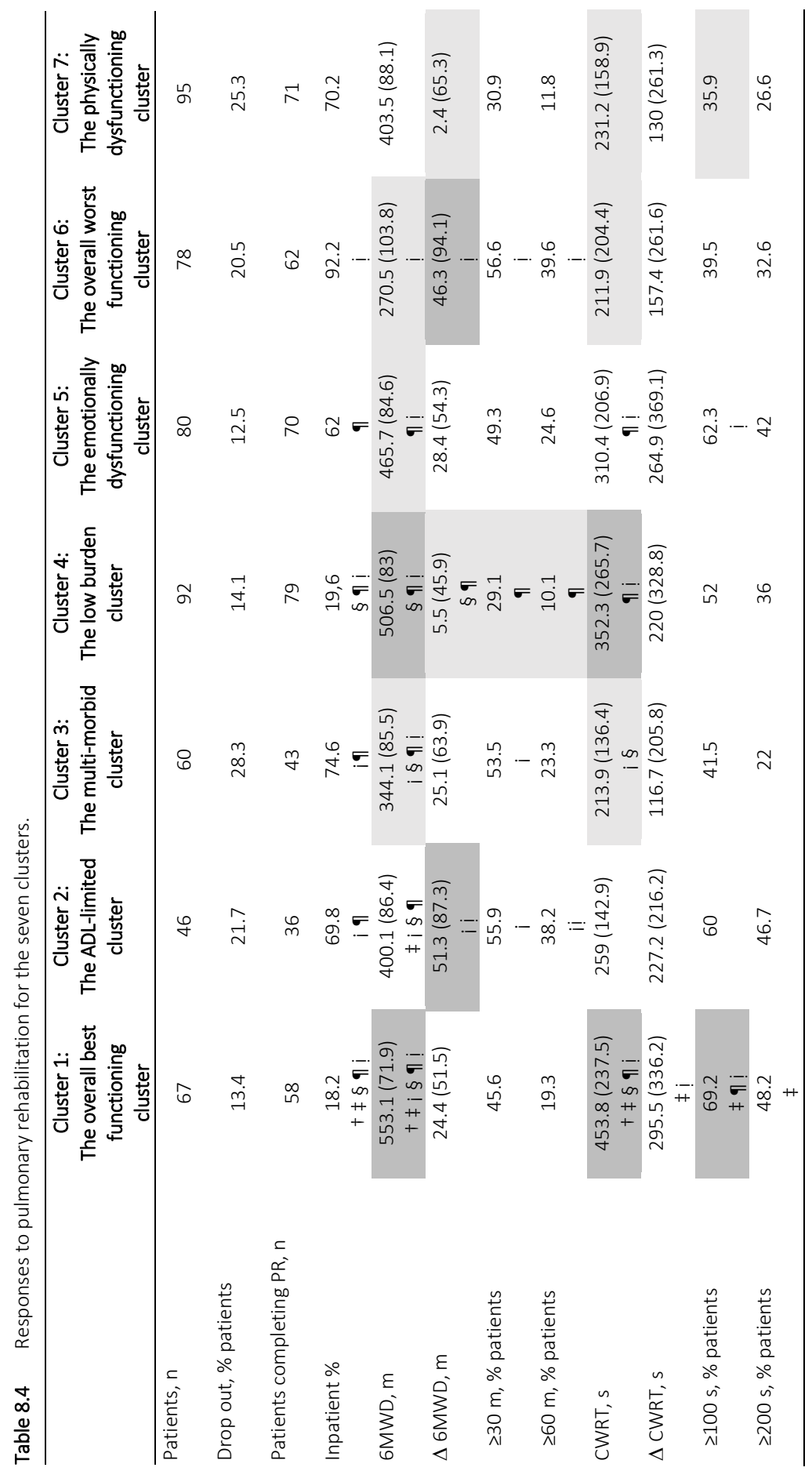




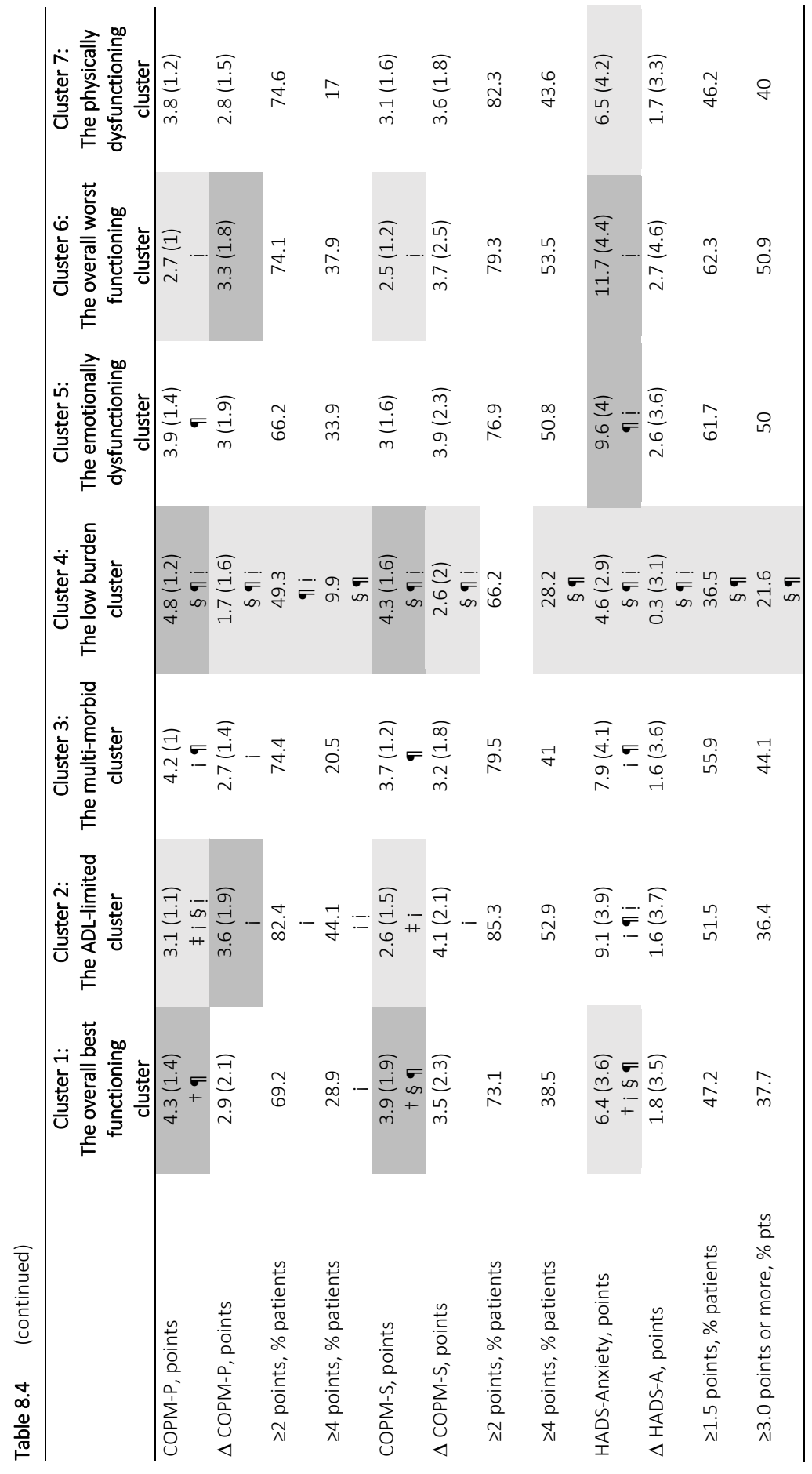




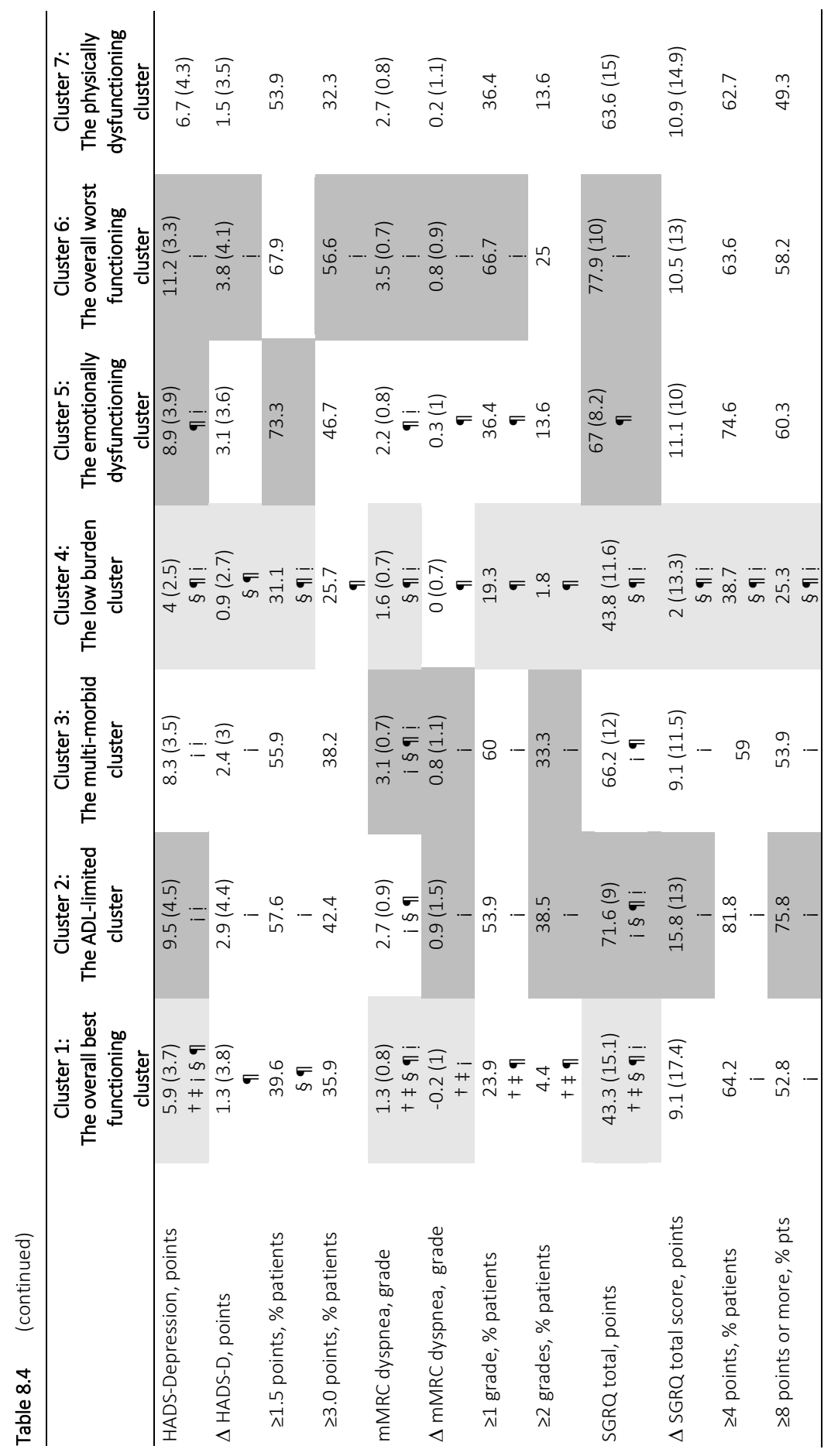




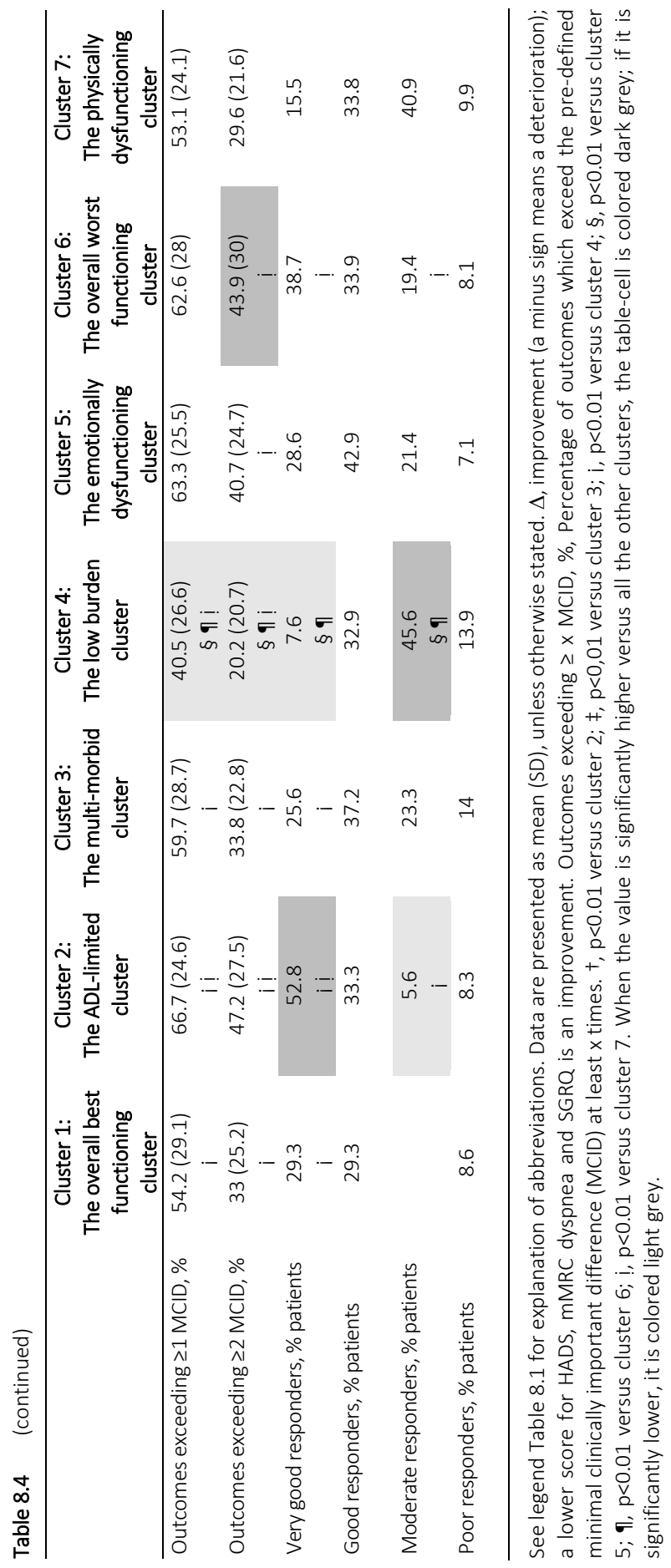


Seven different clusters

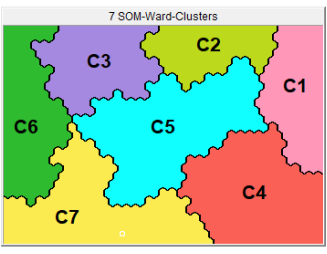

Multidimensional response outcome
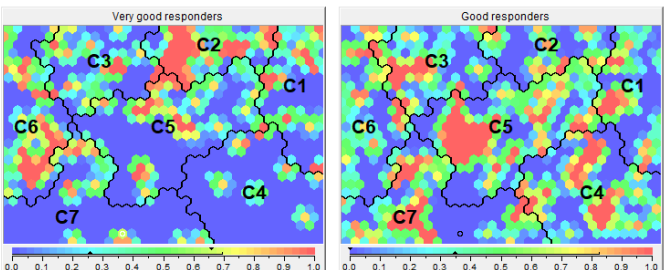

a. Overall response to treatment for the eight response indicators
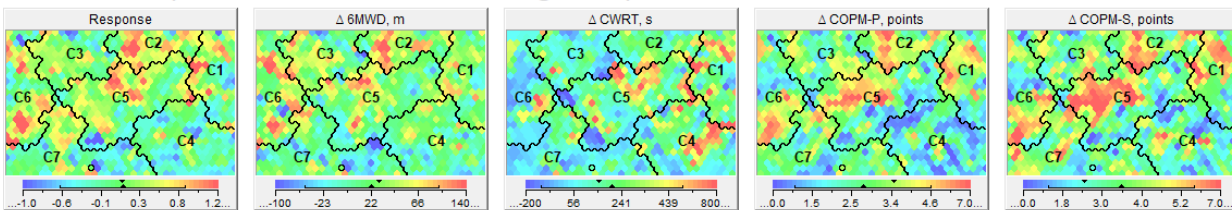

b. Outcomes exceeding at least $1 \mathrm{x}$ minimally clinical important difference
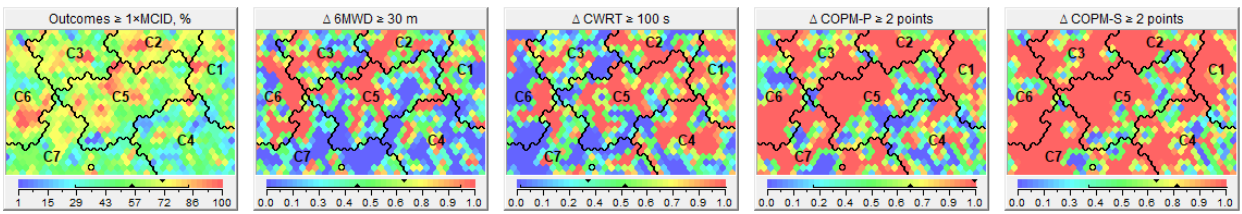

c. Outcomes exceeding at least $2 \mathrm{x}$ minimally clinical important difference
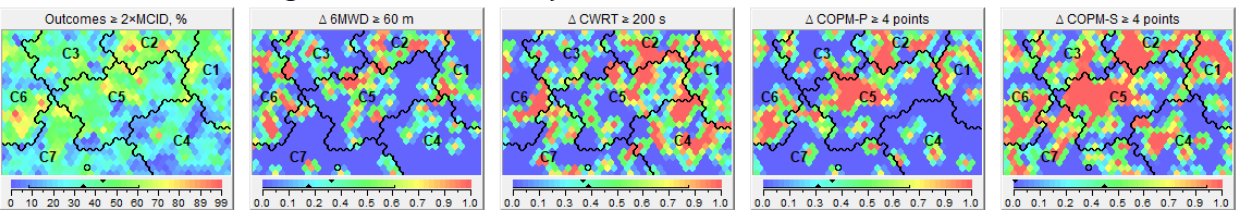

Figure 8.3 Outcomes after pulmonary rehabilitation for the seven traits-based clusters. Panels generated using Viscovery (Viscovery Software $\mathrm{GmbH}$, Vienna, Austria). The seven different traits-based clusters are demonstrated in the upper left corner. The four panels next to it, illustrate (in red) the proportion of very good responders, good responders, moderate responders and poor responders for the different clusters. When looking at the first panel of a) patients "raise a red flag" if they had a very good response, "a green flag" when the response was good to moderate, and "a blue flag" when the response was poor. In this way maps can be interpreted. Refer to the color scale below each attribute picture to match colors with attribute values. All other attribute pictures in a) are the absolute change 

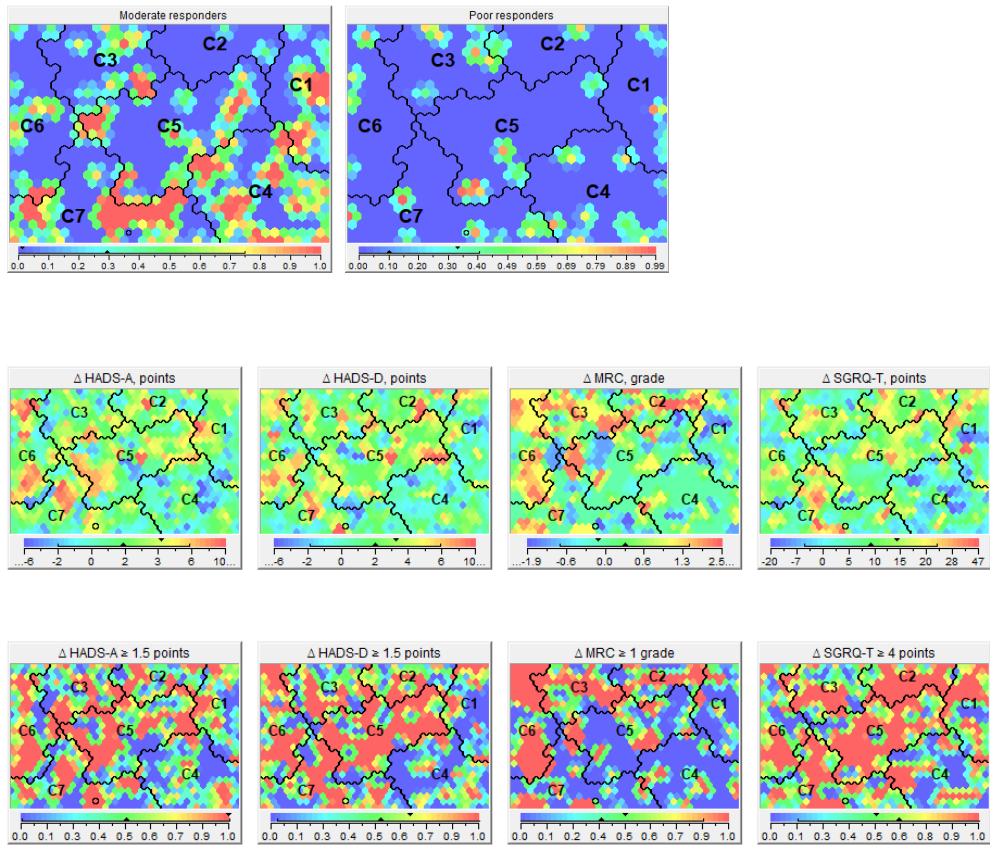

$\triangle$ HADS-A 23 points
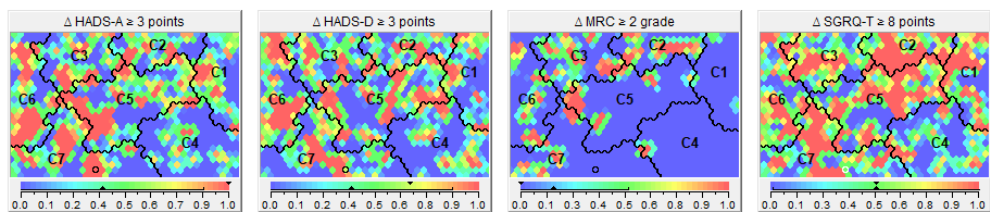

in 6-min walk distance (6MWD), cycle endurance time (constant work-rate test; CWRT), Canadian Occupational Performance Measure, performance (COPM-P), Canadian Occupational Performance Measure, satisfaction (COPM-S), Hospital Anxiety and Depression Scale, anxiety (HADS-A), Hospital Anxiety and Depression Scale, depression (HADS-D), Medical Research Council (MRC) dyspnea grade and St. George's Respiratory Questionnaire total score (SGRQ-T). b) Left panel shows the proportion of clinically relevant outcomes (exceeding at least $1 \times$ minimally clinical important difference (MCID)). All other panels are the proportion of patients per outcome showing a clinically relevant improvement (exceeding at least $1 \times \mathrm{MCID}$ ). c) First panel shows the proportion of clinically relevant outcomes (exceeding at least $2 \times \mathrm{MCID}$ ). All other panels are the proportion of patients per outcome showing a clinically relevant improvement (exceeding at least $2 \times \mathrm{MCID})$. 


\section{Discussion}

The present study confirms the tremendous heterogeneity in pulmonary and extrapulmonary trait impairments in COPD patients referred for PR. The current results corroborate that the response to PR is differential in patients with COPD, which justifies the use of a multidimensional outcome to assess the efficacy of comprehensive PR programs. Furthermore, our study confirms that the differential response to such PR program can be clustered based on multidimensional performance metrics, including evaluation of the functional and emotional daily life disease burden, in identification of groups of patients with very good, good, moderate or poor response. Impaired physical capacity and high disease burden despite better lung function identify the very good responding patients. Clustering based on identified traits may help to identify PR candidates: particularly those patients with low disease burden or overall best functioning are less prone to benefit from PR.

Starting from the first authoritative statement on PR, an accurate diagnosis of the physiopathological and psychopathological manifestations of pulmonary diseases has been put forward. ${ }^{23}$ In the latest definition, PR has been described as a comprehensive, individualized intervention based on a thorough patient assessment. ${ }^{13}$ More recently, in order to realize a personalized or precision medicine approach, the concept of treatable traits has been introduced. $2,24,25$ To cope with these traits, the organization of PR as a flexible, individualized and integrated intervention, based on partnering different skills has been described. ${ }^{14}$ To reflect the complexity and heterogeneity of COPD, we recently described that baseline traits could be grouped into 7 discernible clusters. ${ }^{10}$ Current practice for PR referral is in sharp contrast with this personalized approach and largely ignores this complexity and heterogeneity in impairments. Indeed, the degree of airflow limitation is still predominantly used as criterium to select patients for $\mathrm{PR}^{26,27}$ despite the overwhelming evidence that airflow limitation is a very poor predictor of exercise capacity, physical activity or burden of the disease. ${ }^{28}$ Even clustering of integrated respiratory physiomic characteristics does not allow adequate prediction of $P R$ outcomes. ${ }^{9}$ In the most recent Global Initiative for Obstructive Lung Diseases (GOLD) strategy document, PR is just encouraged for those patients with high symptom burden and at risk for exacerbations. ${ }^{22}$ GOLD recommends a formal rehabilitation program that takes into account the individual characteristics of a COPD patient, however, these characteristics are not further specified.

Our study confirms previous findings that based on a carefully selected set of key performance measures and validated values for MCID, a differential response to PR can be demonstrated varying from very good responders to poor responders. ${ }^{4}$ Previous studies reported that individual patients respond differentially on various types of outcomes. ${ }^{29,30}$ In the group of disabled COPD patients of the current study more than $50 \%$ improved health status with at least 2 times the MCID while most evidence-based 
pharmacological interventions showed marginally clinical relevant differences. ${ }^{31}$ Very good responders demonstrated the highest disease burden, manifested by lower exercise performance, worse scores on activities in daily living, anxiety, depression and quality of life although they had better pulmonary traits.

Our data even suggest the role of the current intervention in the reduction of anxiety and depression. ${ }^{32}$ Previous data reported that coping styles and/or changes in coping styles are related to changes in anxiety and depression after PR and that good responders particularly decreased their passive coping style. ${ }^{33}$ At least our data illustrate that in the majority of the patients PR improves the physical, emotional and social functioning of the patient. ${ }^{34}$ This study confirms previous findings showing that sex, age, the degree of airflow limitation or even ambulatory oxygen therapy cannot be used to predict outcomes of PR. ${ }^{4}$ Although not systematically explored, it seems that patients in the cluster with a higher percentage of drop-out, largely related to interfering exacerbations, were even more impaired in physical capacity and disease burden than the other clusters.

Although in- and outpatient programs are matched in terms of the composition and scheduled interventions, the number of very good and good responders is remarkably higher after inpatient rehabilitation. Inpatient rehabilitation was based on the interdisciplinary evaluation of care dependency and needs for extensive medical supervision. We previously reported the need for more holistic, personalized approaches to optimize the patient's quality of life integrating the patient's whole environment and the team of health care professionals in co-creating value care. ${ }^{35}$ It is now well recognized that patient's personalities, health beliefs, social support networks, financial resources and other unique life circumstances have important effects how an individual patient respond to treatment. ${ }^{35}$ These context- and program-based influences have been largely neglected in previous selection criteria for PR settings in COPD patients. ${ }^{36}$ Further studies are needed to explore this co-creating value in health caring of inpatient rehabilitation. ${ }^{37}$

Identification of patients or clusters of patients that do or do not respond to PR will be an important step to improve the cost-effectiveness of PR. Based on a thorough assessment of a broad trait panel and applying a multidimensional set of key performance measures, this study aimed to evaluate the outcome predictability of previously identified clusters of COPD. ${ }^{10}$ At least 2 clusters with attenuated response to PR could be identified, the so-called overall best functioning cluster and the cluster with overall low disease burden. Remarkable are the effects of PR in the overall worst functioning and the physically dysfunctioning cluster, clusters of disabled patients: 58 and $49 \%$ of these patients improved health status with at least 2 times the minimally clinically defined difference. Despite these cluster differences, our data underscores the 
need for a personalized trait profile and checking individual management goals at the start of a PR program.

Furthermore, while PR is generally advocated as a standard of care to improve shortness of breath, health status and exercise tolerance ${ }^{38}$, our data suggest a shift towards performance and satisfaction of daily life activities as well as towards attenuation of the emotional and overall burden of the disease. ${ }^{4,26}$ Indeed, current guidelines focus on symptom and risk reduction as PR outcomes in line with outcomes of pharmacological interventions, while by definition PR has broader aims. ${ }^{13}$ Therefore, it will become important to realize that for COPD patients as well as for chronic patients in general a more dynamic vision on outcomes will be needed considering health as a state of wellbeing characterized by the physical, mental and social potential. ${ }^{39,40}$ PR as comprehensive management must address the needs of the patients by evidence-based and efficient interventions in combination with health caring in response with the feelings that matter for the patient. ${ }^{37}$ Besides physical training, PR aims to create continuous healing relationships, customized according the patients' needs and values and in partnership with the patient. ${ }^{41}$ In this way PR has the potential to improve resilience in the different health domains as well as the individual's well-being. ${ }^{39,40}$ This approach conducted in specialized PR programs is completely different from exercisebased care programs, generally described also as PR interventions.

The present results are observational and definitive conclusions need to be based on further prospective data. At least, the current results aim to contribute to repositioning of PR as a comprehensive, personalized intervention tackling the multiple physical, emotional and/or social treatable traits of the referred patient and to stimulate the discussions on real patient-related outcomes. Considering this complexity, the organization of PR is more than scheduling trait based interventions, but needs to tackle this heterogenous burden of the disease. ${ }^{34}$ The strength of this study is the in-depth assessment of a wide set of traits, the supervision of the whole rehabilitation program as well as the broad outcome evaluation by independent technicians. One of the weaknesses of the study is of course that the data are derived from a single center for PR, hampering the generalization of our findings, and that no follow-up data are available. Another potential limitation of this study could be that the PR program is conducted according the 2013 ATS/ERS recommendations on PR. ${ }^{13}$ At that time, the scope of PR was more directed on symptom relief and on exercise intolerance in particular rather than on the emotional and social domains. Furthermore, the current multidimensional response profiling is based on a set of eight expert-opinion outcome measures, with allocating the greatest importance to physical performance. More concise patient-derived and -related outcomes need to be developed in the future to better describe the dynamic changes of health transition in these patients. 
To conclude, the current study confirms the differential response to PR based on multidimensional response profiling and provides detailed insights in trait complexity and performance metrics in patients referred for PR. Cluster analysis of baseline traits illustrates that clinically important differences can be achieved in the most functionally frail and emotionally impaired clusters and that the overall best functional as well as the low burden cluster manifested an attenuated outcome. The time has come to start up the discussion how PR will be re-organized not to reduce impairments or symptoms but to improve the functional, emotional and social domains of health in patients with COPD and other chronic respiratory conditions. 


\section{References}

1. Wouters EFM, et al. Personalised pulmonary rehabilitation in COPD. Eur Respir Rev. 2018;27(147).

2. Agusti A, et al. Treatable traits: toward precision medicine of chronic airway diseases. Eur Respir J. 2016; 47(2):410-9.

3. Spruit MA, et al. Differences in content and organisational aspects of pulmonary rehabilitation programmes. Eur Respir J. 2014;43(5):1326-37.

4. Spruit MA, et al. Differential response to pulmonary rehabilitation in COPD: multidimensional profiling. Eur Respir J. 2015;46(6):1625-35.

5. Spruit MA, et al. Profiling of Patients with COPD for Adequate Referral to Exercise-Based Care: The Dutch Model. Sports Med. 2020;50(8):1421-9.

6. Vanfleteren LE, et al. Clusters of comorbidities based on validated objective measurements and systemic inflammation in patients with chronic obstructive pulmonary disease. Am J Respir Crit Care Med. 2013; 187(7):728-35.

7. Augustin IML, et al. The respiratory physiome: Clustering based on a comprehensive lung function assessment in patients with COPD. PLoS One. 2018;13(9):e0201593.

8. Mesquita R, et al. Objectively identified comorbidities in COPD: impact on pulmonary rehabilitation outcomes. Eur Respir J. 2015;46(2):545-8.

9. Augustin IML, et al. Comprehensive Lung Function Assessment Does not Allow to Infer Response to Pulmonary Rehabilitation in Patients with COPD. J Clin Med. 2018;8(1).

10. Augustin IML, et al. Incorporating Comprehensive Assessment Parameters to Better Characterize and Plan Rehabilitation for Persons with Chronic Obstructive Pulmonary Disease. J Am Med Dir Assoc. 2020;

11. Smid DE, et al. Impact of cardiovascular comorbidities on COPD Assessment Test (CAT) and its responsiveness to pulmonary rehabilitation in patients with moderate to very severe COPD: protocol of the Chance study. BMJ Open. 2015;5(7):e007536.

12. Spruit MA, et al. Integration of pulmonary rehabilitation in COPD. Lancet. 2008;371(9606):12-3.

13. Spruit MA, et al. An official American Thoracic Society/European Respiratory Society statement: key concepts and advances in pulmonary rehabilitation. Am J Respir Crit Care Med. 2013;188(8):e13-64.

14. Augustin I, Wouters E. Process of pulmonary rehabilitation and program organization. J Card Pulm Rehabil. 2017;1:109.

15. Sillen MJ, et al. Efficacy of lower-limb muscle training modalities in severely dyspnoeic individuals with COPD and quadriceps muscle weakness: results from the DICES trial. Thorax. 2014;69(6):525-31.

16. Mahler DA, Wells CK. Evaluation of clinical methods for rating dyspnea. Chest. 1988;93(3):580-6.

17. Jones PW, Quirk FH, Baveystock CM. The St George's Respiratory Questionnaire. Respir Med. 1991;85 Suppl B:25-31; discussion 33-7.

18. Holland AE, et al. An official European Respiratory Society/American Thoracic Society technical standard: field walking tests in chronic respiratory disease. Eur Respir J. 2014;44(6):1428-46.

19. van 't Hul AJ, Gosselink R, Kwakkel G. Constant-load cycle endurance performance: test-retest reliability and validity in patients with COPD. J Cardiopulm Rehabil. 2003;23:143-50.

20. Annegarn J, et al. Problematic activities of daily life are weakly associated with clinical characteristics in COPD. J Am Med Dir Assoc. 2012;13(3):284-90.

21. Snaith RP. The Hospital Anxiety And Depression Scale. Health Qual Life Outcomes. 2003;1:29.

22. GOLD. Global Strategy for Prevention, Diagnosis and management of COPD. 2021.

23. Hodgkin JE, et al. American Thoracic Society. Medical Section of the American Lung Association. Pulmonary rehabilitation. Am Rev Respir Dis. 1981;124(5):663-6.

24. McDonald VM, et al. Treatable traits: a new paradigm for 21st century management of chronic airway diseases: Treatable Traits Down Under International Workshop report. Eur Respir J. 2019;53(5).

25. Fingleton J, Hardy J, Beasley R. Treatable traits of chronic airways disease. Curr Opin Pulm Med. 2018; 24(1):24-31.

26. McCarthy B, et al. Pulmonary rehabilitation for chronic obstructive pulmonary disease. Cochrane Database Syst Rev. 2015(2):CD003793.

27. Qaseem A, et al. Diagnosis and management of stable chronic obstructive pulmonary disease: a clinical practice guideline update from the American College of Physicians, American College of Chest Physicians, American Thoracic Society, and European Respiratory Society. Ann Intern Med. 2011;155(3):179-91. 
28. Curtis JR, Deyo RA, Hudson LD. Pulmonary rehabilitation in chronic respiratory insufficiency. 7. Healthrelated quality of life among patients with chronic obstructive pulmonary disease. Thorax. 1994;49(2): 162-70.

29. de Torres JP, et al. Power of outcome measurements to detect clinically significant changes in pulmonary rehabilitation of patients with COPD. Chest. 2002;121(4):1092-8.

30. Crisafulli $E$, et al. Role of comorbidities in a cohort of patients with COPD undergoing pulmonary rehabilitation. Thorax. 2008;63(6):487-92.

31. Cope $\mathrm{S}$, et al. Comparative efficacy of long-acting bronchodilators for COPD: a network meta-analysis. Respir Res. 2013;14:100.

32. Farah WH, et al. Non-pharmacological treatment of depression: a systematic review and evidence map. Evid Based Med. 2016;21(6):214-21.

33. Stoilkova-Hartmann A, et al. Differences in change in coping styles between good responders, moderate responders and non-responders to pulmonary rehabilitation. Respir Med. 2015;109(12):1540-5.

34. Wouters EF, Augustin IM. COPD health-care delivery: a holistic and dynamic approach is needed. Lancet Respir Med. 2016;4(6):e30-1.

35. Houben-Wilke S, et al. COPD stands for complex obstructive pulmonary disease. Eur Respir Rev. 2018; 27(148).

36. Donner CF, Muir JF. Selection criteria and programmes for pulmonary rehabilitation in COPD patients. Rehabilitation and Chronic Care Scientific Group of the European Respiratory Society. Eur Respir J 1997; 10(3):744-57.

37. Chochinov HM. Health care, health caring, and the culture of medicine. Curr Oncol. 2014;21(5):e668-9.

38. Ries AL, et al. Pulmonary Rehabilitation: Joint ACCP/AACVPR Evidence-Based Clinical Practice Guidelines. Chest. 2007;131(5 Suppl):4S-42S.

39. Huber M, et al. How should we define health? BMJ. 2011;343:d4163.

40. Bircher J. Towards a dynamic definition of health and disease. Med Health Care Philos. 2005;8(3):335-41.

41. Institute of Medicine, Crossing the Quality Chasm: A New Health System for the 21st Century. 2001: Washington, DC: The National Academies Press. 


\section{Supplemental material S8.1: Eligibility criteria for Chance study}

Eligibility criteria are described in Smid, D.E., et al., Impact of cardiovascular comorbidities on COPD Assessment Test (CAT) and its responsiveness to pulmonary rehabilitation in patients with moderate to very severe COPD: protocol of the Chance study. BMJ Open, 2015. 5(7): p. e007536. ${ }^{1}$

Patients were recruited by a specialized pulmonary rehabilitation centre. The inclusion of subjects started in April 2012. The inclusion of the subjects from the tertiary care setting has been completed mid-2014. These were patients with COPD referred for clinical assessment and pulmonary rehabilitation to Ciro, Horn, The Netherlands.

Patients were eligible if they fulfilled the following criteria:

1. Age 40-85 years.

2. A diagnosis of COPD according to GOLD guidelines.

3. Referral for assessment and pulmonary rehabilitation in Ciro by a chest physician.

Exclusion criteria for the patients with COPD:

1. A history of asthma, lung cancer, sarcoidosis, tuberculosis, lung fibrosis, cystic fibrosis or any other significant respiratory disease.

2. A moderate or severe exacerbation or pneumonia requiring systemic corticosteroids, antibiotics or hospitalisation during the last 4 weeks.

3. Having undergone lung surgery (e.g. lung volume reduction, lung transplantation).

4. Any clinically relevant disease which in the opinion of the investigator may influence the results of the study.

5. Malignancy within the last 5 years.

6. For primary care patients: treatment by respiratory physician in secondary or tertiary care.

For secondary care patients: treatment in tertiary care setting in the previous 5 years.

1. Smid, D.E., et al., Impact of cardiovascular comorbidities on COPD Assessment Test (CAT) and its responsiveness to pulmonary rehabilitation in patients with moderate to very severe COPD: protocol of the Chance study. BMJ Open. 2015;5(7):e007536. 


\section{Supplemental material S8.2: Measurements and statistics of the previously identified clusters}

Clusters are described in detail in Augustin, I.M.L., et al., Incorporating Comprehensive Assessment Parameters to Better Characterize and Plan Rehabilitation for Persons with Chronic Obstructive Pulmonary Disease. J Am Med Dir Assoc, 2020. ${ }^{1}$

\section{Measurements}

As described previously ${ }^{1,2}$, the pre-rehabilitation assessment includes the identification of pulmonary and extra-pulmonary (functional, behavioral and health status) attributes.

Pulmonary traits were determined by a comprehensive lung function assessment and existed of airflow limitation, static hyperinflation, gas transfer, respiratory pressures and arterial blood gases. Post-bronchodilator spirometry was performed to assess forced expiratory volume in 1 second $\left(F V_{1}\right)$ and forced vital capacity (FVC). Spirometry was measured with Masterlab ${ }^{\circledR}$ (Jaeger, Würzburg, Germany) following ATS/ERS guidelines. ${ }^{3}$ Values are expressed as percentage of predicted according the Global Lung Function Initiative. ${ }^{4}$ Total lung capacity (TLC), residual volume (RV) and intra thoracic gas volume (ITGV) were determined through body-plethysmography (Masterlab ${ }^{\circledR}$ Jaeger, Würzburg, Germany) following the quality control guidelines. ${ }^{5}$ Values are expressed as a percentage of the European Coal and Steel Community predicted values. ${ }^{6}$ Transfer factor of the lung for carbon monoxide (TLCO) was measured following the standard of the single-breath determination of carbon monoxide ${ }^{7}$ and expressed in the reference values of Cotes and colleagues. $^{8}$ Additionally, TLCO per unit alveolar volume (KCO) was calculated. Maximal static inspiratory (MIP) and expiratory mouth pressures (MEP) were assessed according to ATS/ERS guidelines ${ }^{9}$ and expressed in the reference values according to Black and Hyatt. ${ }^{10}$ Resting arterial partial pressure of oxygen $\left(\mathrm{PaO}_{2}\right)$, carbon dioxide $\left(\mathrm{PaCO}_{2}\right)$ and oxygen saturation were measured (GEM4000, Instrumentation Laboratory, Peachtree City, USA). Patients with long term oxygen therapy (LTOT) continued oxygen supply during the procedure. All lung function measurements were performed by certified and experienced respiratory technicians.

Measured functional extra-pulmonary traits (physical) were muscle strength, physical functioning, body composition and presence of comorbidities. Isokinetic quadriceps peak torque was measured using a Biodex (Biodex Medical Systems, Inc., New York, USA). ${ }^{11,12}$ Exercise performance was assessed by a 6 -minute walk test (6MWT) performed according to international guidelines. ${ }^{13}$ The longest distance covered in two separate tests was registered as the 6-minute walk distance (6MWD). Furthermore, a symptom-limited incremental cardiopulmonary exercise test (CPET) was performed using an electrically, braked cycle ergometer (Carefusion, Houten, the Netherlands) including the measurement of maximal oxygen uptake $\left(\right.$ Peak $\left.\mathrm{VO}_{2}, \mathrm{ml} / \mathrm{min}\right)$ and maximal 
work rate in Watts (Peak work rate). ${ }^{14}$ Endurance exercise capacity was measured by a sub-maximal exercise test at $75 \%$ of the peak work rate (CWRT). ${ }^{15}$ Body mass index (BMI) was calculated by body weight in $\mathrm{kg} /$ (height in $\mathrm{m})^{2}$. Fat-free mass (FFM) was assessed using dual-energy X-ray absorptiometry (DEXA) scan (Lunar Prodigy scan, GE Healthcare, Madison, WI, USA) and FFM (=lean mass + bone mineral content) was divided by squared height (in $\mathrm{m}$ ) to obtain the FFM-index (FFMI). ${ }^{16}$ Bone mineral density (BMD by DEXA scan) was measured at the hip, lumbar spine, and whole body. ${ }^{17}$ Selfreported comorbidities were assessed using the Charlson Comorbidity Index (CCI). ${ }^{18}$

Extra-pulmonary behavioral traits reflected by social functioning and emotional function, symptom perception and health status were assessed. The Care Dependency Scale (CDS), consisting of 15 items regarding basic and instrumental activities of daily living, was used to assess the level of care dependency. ${ }^{19}$ The Canadian Occupational Performance Measure (COPM) was used to identify specific problematic activities of daily life. ${ }^{20}$ Patients scored how well they were performing the problematic activities of daily life (performance score; COPM-P) and how satisfied they were with this level of performance (satisfaction score; COPM-S). Timed Up and Go (TUG) test was assessed to measure functional mobility. ${ }^{21}$ Emotional functioning has been measured using the Hospital Anxiety and Depression Scale (HADS) ${ }^{22}$, which is divided into an anxiety subscale (HADS-A) and a depression subscale (HADS-D). The degree of dyspnea was measured using the modified Medical Research Council (mMRC) scale. ${ }^{23}$ The disease-specific health status was assessed using the COPD Assessment Test (CAT) ${ }^{24}$, a simple questionnaire to measure the impact of COPD on a person's life, and using the COPD-specific version of the St George's Respiratory Questionnaire (SGRQ-C) ${ }^{25}$ that consists of three domains scores (symptoms, activity and impact) and a total score. Furthermore, the Clinical COPD Questionnaire (CCQ), a self-administered questionnaire consisting of three domains specially developed to measure clinical control in patients with COPD, was used. ${ }^{26}$

In addition the assessment included demographics, medical history, the number of exacerbations and hospitalizations for COPD in the last twelve months, and the use of long-term oxygen (LTOT). Patients with COPD were classified as Global Initiative for Chronic Obstructive Lung Disease (GOLD) I (mild $=\mathrm{FEV}_{1} \geq 80 \%$ predicted), II (moderate $=$ $50 \% \leq \mathrm{FEV}_{1}<80 \%$ predicted), III (severe $=30 \% \leq \mathrm{FEV}_{1}<50 \%$ ), IV (very severe $=\mathrm{FEV}_{1}<30 \%$ predicted) and GOLD A (mMRC 0-1, CAT < 10 and 0 or 1 exacerbation not leading to hospital admission), $B$ ( $m M R C \geq 2, C A T \geq 10$ and 0 or 1 exacerbation not leading to hospital admission), C (mMRC 0-1, CAT $<10$ and $\geq 2$ or $\geq 1$ exacerbation leading to hospital admission), $\mathrm{D}$ ( $\mathrm{mMRC} \geq 2, \mathrm{CAT} \geq 10$ and $\geq 2$ or $\geq 1$ exacerbation leading to hospital admission). ${ }^{27}$

\section{Statistics}

Self-organizing maps (SOMs, also referred to as Kohonen maps) were used to create an ordered representation of the selected attributes. The SOM method can be viewed as a 
non-parametric regression technique that simplifies complexity by converting multidimensional data spaces into lower dimensional abstractions. A SOM generates a non-linear representation of the data distribution and allows the user to identify homogeneous data groups visually to reveal meaningful relationships. All statistical analyses were performed using Viscovery SOMine 7.1 by Viscovery Software GmbH (http://www.viscovery.net, Vienna, Austria).

Patients were ordered by their overall similarity concerning the attributes of the prerehabilitation assessment, which are the following in descending order of priority: $\mathrm{FEV}_{1} / \mathrm{FVC}$ in \%; FEV $1, F V C$, PEF, ITGV, RV,TLC TLCO, KCO, MIP and MEP (all in \% predicted); mMRC dyspnea grade; CAT, total score; SGRQ, total score; CCQ, total score; CDS, item daily activities in points and CDS, total points; COPM-P in points; 6MWD in m; Peak work rate in Watts; Quadriceps peak torque in \% predicted; TUG test in S; FEV in L; $_{\text {; }}$ Airway resistance effective measured during bodyplethysmography in $\mathrm{KPa}$ *sec/L; COPD GOLD I-IV; HADS-D in points; Users of rollator in \%; HADS-A in points; CAT score section impact on activities; CDS, item mobility; 6MWD in \% predicted; LTOT users in \%; CDS, item eating and drinking in points; CDS, item getting dressed and undressed in points; Quadriceps muscle endurance (Total Work in Joules); SGRQ score, section activity in points; SGRQ score, for section impact in points; COPD related hospital admissions last 12 months; SGRQ score, section symptom in points; arterial blood gases $\left(\mathrm{PaCO}_{2}, \mathrm{CHCO}_{3}\right.$, $\mathrm{PaO}_{2}, \mathrm{SaO}_{2}$ ) and $\mathrm{CCQ}$ scores for functional state; and to a small extent absolute measures of FVC; PEF; ITGV; RV; TLC; TLCO; KCO; TLC by helium dilution technique; inspiratory vital capacity (IC); breath holding time (TA); MIP; and MEP.

Based on the created SOM model, clusters were generated using the SOM-Ward Cluster algorithm of Viscovery, a hybrid algorithm that applies the classical hierarchical method of Ward on top of the SOM topology.

\section{Supplemental references}

1. Augustin IML, et al. Incorporating Comprehensive Assessment Parameters to Better Characterize and Plan Rehabilitation for Persons with Chronic Obstructive Pulmonary Disease. J Am Med Dir Assoc. 2020.

2. Augustin IML, et al. The respiratory physiome: Clustering based on a comprehensive lung function assessment in patients with COPD. PLoS One. 2018;13(9):e0201593.

3. Miller MR, et al. Standardisation of spirometry. Eur Respir J. 2005;26(2):319-38.

4. Quanjer PH, et al. Multi-ethnic reference values for spirometry for the 3-95-yr age range: the global lung function 2012 equations. Eur Respir J. 2012;40(6):1324-43.

5. Coates AL, et al. Measurement of lung volumes by plethysmography. Eur Respir J. 1997;10(6):1415-27.

6. Quanjer PH, et al. Lung volumes and forced ventilatory flows. Eur Respir J. 1993;6 Suppl 16:5-40.

7. Macintyre $\mathrm{N}$, et al. Standardisation of the single-breath determination of carbon monoxide uptake in the lung. Eur Respir J. 2005;26(4):720-35.

8. Cotes JE, et al. Standardization of the measurement of transfer factor (diffusing capacity). Eur Respir J. 1993;6 Suppl 16:41-52. 
9. American Thoracic Society/European Respiratory S, ATS/ERS Statement on respiratory muscle testing. Am J Respir Crit Care Med. 2002;166(4):518-624.

10. Black LF, Hyatt RE. Maximal respiratory pressures: normal values and relationship to age and sex. Am Rev Respir Dis. 1969;99(5):696-702.

11. Borges O. Isometric and isokinetic knee extension and flexion torque in men and women aged 20-70. Scand J Rehabil Med. 1989;21(1):45-53.

12. Ribeiro F, et al. Test-retest reliability of lower limb isokinetic endurance in COPD: A comparison of angular velocities. Int J Chron Obstruct Pulmon Dis. 2015;10:1163-72.

13. Holland $A E$, et al. An official European Respiratory Society/American Thoracic Society technical standard: field walking tests in chronic respiratory disease. Eur Respir J. 2014;44(6):1428-46.

14. American Thoracic S, P. American College of Chest, ATS/ACCP Statement on cardiopulmonary exercise testing. Am J Respir Crit Care Med. 2003;167(2):211-77.

15. van 't Hul A, Gosselink R, Kwakkel G. Constant-load cycle endurance performance: test-retest reliability and validity in patients with COPD. J Cardiopulm Rehabil. 2003;23(2):143-50.

16. Schols AM, et al. Nutritional assessment and therapy in COPD: a European Respiratory Society statement. Eur Respir J. 2014;44(6):1504-20.

17. Graat-Verboom L, et al. Whole-Body versus Local DXA-Scan for the Diagnosis of Osteoporosis in COPD Patients. J Osteoporos. 2010;2010:640878.

18. Charlson ME, et al. A new method of classifying prognostic comorbidity in longitudinal studies: development and validation. J Chronic Dis. 1987;40(5):373-83.

19. Dijkstra A, et al. Further psychometric testing of the Dutch Care Dependency Scale on two different patient groups. Int J Nurs Pract. 2002;8(6):305-14.

20. Annegarn J, et al. Problematic activities of daily life are weakly associated with clinical characteristics in COPD. J Am Med Dir Assoc. 2012;13(3):284-90.

21. Mesquita R, et al. Within-day test-retest reliability of the Timed Up \& Go test in patients with advanced chronic organ failure. Arch Phys Med Rehabil. 2013;94(11):2131-8.

22. Zigmond AS, Snaith RP. The hospital anxiety and depression scale. Acta Psychiatr Scand. 1983;67(6):361-70.

23. Mahler DA, Wells CK. Evaluation of clinical methods for rating dyspnea. Chest. 1988;93(3):580-6.

24. Jones PW, et al. Development and first validation of the COPD Assessment Test. Eur Respir J. 2009;34(3): 648-54.

25. Jones PW, Quirk FH, Baveystock CM. The St George's Respiratory Questionnaire. Respir Med. 1991;85 Suppl B:25-31; discussion 33-7.

26. van der Molen $\mathrm{T}$, et al. Development, validity and responsiveness of the Clinical COPD Questionnaire. Health Qual Life Outcomes. 2003;1:13.

27. GOLD. Global Strategy for Prevention, Diagnosis and management of COPD. 2021. 




\section{Chapter 9}

Summary and general discussion 



\section{Summary and general discussion}

Nowadays, COPD (chronic obstructive pulmonary disease) is considered a complex syndrome with multiple pulmonary and extra-pulmonary features. ${ }^{1,2}$ Despite this growing understanding of the disease's complexity, many factors that affect the health status and burden of disease in the individual patient remain undiagnosed and undertreated. ${ }^{3}$ Historically, pulmonary rehabilitation addresses the physiopathological and psychopathological problems that remain after optimal pharmacological treatment in an individualized program based on a thorough assessment (see Chapters 1 and 3). ${ }^{4}$ Absence of consensus on the components and domains of this assessment significantly contributes to the currently existing heterogeneity in pulmonary rehabilitation programs (see Chapter 1 ).

The heterogeneity of COPD with respect to clinical presentation, physiology, imaging, response to therapy, decline in lung function and survival is widely recognized in COPD literature. ${ }^{1}$ The identification and grouping of key elements of the COPD syndrome into clinically meaningful and useful subgroups that can guide therapy more effectively has been introduced as a potential solution to tackle this heterogeneity. Unfortunately, in COPD research, operational definitions are applied to define these possible subgroups or so-called phenotypes. While a phenotype by definition reflects the observable structural and functional characteristics of an organism determined by its genotype and modulated by its environment ${ }^{5}$, in the operational COPD approach, phenotypes should enable the classification of COPD patients into distinct subgroups that provide prognostic information, allowing better determination of appropriate therapy and altering clinically meaningful outcomes. ${ }^{1}$ This clinical perspective on so-called phenotypes can be illustrated by the introduction of the frequent exacerbator ${ }^{6}$ or the upper lobe emphysema phenotypes. ${ }^{7}$ Furthermore, meaningful outcomes are limited to symptoms, exacerbations, rate of disease progression or death. ${ }^{1}$ Pulmonary and extra-pulmonary components of COPD are even defined as disease traits and randomly addressing these traits as precision medicine. ${ }^{8}$

The main objective of this thesis was to identify, based on these traits, clusters in patients with COPD referred for pulmonary rehabilitation as well as to assess differential outcomes in these pre-rehabilitation traits-based clusters in order to structure treatment interventions. This chapter aims to position our results in perspective.

\section{Towards a control panel for COPD?}

In an attempt to describe expectations about the future of medicine and health care and driven by the intrinsic motivation to correct abnormal functioning of the body or bodily compartments, concepts such as "precision medicine", "stratified medicine", "systems medicine", "P4 medicine" and "personalized medicine" have been proposed within 
plural contexts. ${ }^{9}$ While all terms refer to the grouping of patients based on risk of disease or response to therapy using diagnostic tests or techniques ${ }^{10}$, these concepts are used interchangeably and frequently not very strictly. This also seems to be the case in COPD literature. Highlighting the limitations of stratified medicine (phenotype-based), a COPD control panel which included at least the severity, activity and impact domains of the COPD disease was put forward to make progress in personalized medicine. ${ }^{11,12}$ Each of these domains must present information on different aspects of the disease with potential prognostic value and/or with specific therapeutic requirements. Just as pilots use one to fly planes safely, such a control panel must allow the visualization of the status of the relevant domains of the disease for doctors caring for patients with COPD to make appropriate therapeutic decisions. Patients are expected to behave like a plane, and personalized was seen as the integration of information coming from these various sources. ${ }^{12}$ In this approach, the pilot neglects to check the reliability of the control panel to enable him or her to safely fly the plane, to assess the stimulus-response variability of the different gauges on the dashboard and to check the destination with the passengers. Later on, the respiratory community embraced a "label-free" precision medicine strategy for the management of patients with airway disease that was based on the identification of "treatable traits" in each patient. 8,13 This strategy is introduced as a new paradigm for the management of complex airway disease, applying personalized medicine to each individual to improve outcomes. ${ }^{13}$

In fact, this individualized approach has already formed the cornerstone of current pulmonary rehabilitation practice for many years (see Chapter 3). ${ }^{4}$ Indeed, pulmonary rehabilitation starts with a multidimensional assessment consisting of the identification of pulmonary treatable traits, extra-pulmonary functional/behavioral treatable traits and health status in order to individualize the treatment to the needs of the patient with complex COPD (see Chapter 3). ${ }^{4,14}$ In this thesis, we clearly illustrate that neither clustering based on comprehensive lung function measurements (see Chapter 4$)^{15}$ nor clustering based on pulmonary or extra-pulmonary traits and health status (see Chapter $7)^{16}$ has led to clearly discernible subgroups of patients. Our findings in Chapter $4^{15}$ clearly illustrate that a simple classification of COPD based on spirometry alone really underestimates the heterogeneity in respiratory impairment at referral. Based on postbronchodilator spirometry, diffusing capacity of the lung for carbon monoxide, whole body plethysmography, mouth pressures, and arterial blood gases, seven lung function clusters could be distinguished: 1 ) patients with mild to moderate airflow limitation with mildly impaired diffusing capacity, 2) moderate to severe airflow limitation with moderately impaired diffusing capacity and respiratory muscle weakness, 3) moderate to severe airflow limitation with moderately impaired diffusing capacity, 4) moderate to severe airflow limitation with mildly impaired diffusing capacity, 5) severe airflow limitation with severe static hyperinflation and moderately impaired diffusing capacity and respiratory muscle weakness, 6) severe airflow limitation with severe static hyperinflation and moderately impaired diffusing capacity and 7) severe airflow 
limitation with severe static hyperinflation and severely impaired diffusing capacity with respiratory muscle weakness and alveolar hypoventilation (Chapter 4). ${ }^{15}$ The tremendous overlap between these clusters limits predictability when it comes to the individual patient. Although comprehensive lung function has to be part of an initial assessment to understand the pathophysiological respiratory impairments, Chapter $4^{15}$ also demonstrates that even profound assessment and clustering of measurable lung variables poorly predict functional performance and health status in these patients. Chapter $7^{16}$ summarizes the results of clustering based on respiratory, functional and emotional traits. Seven patient profiles are discerned: 1) 'the overall best-functioning' cluster; 2) 'the ADL-limited' cluster: 3) 'the multi-morbid' cluster, 4) 'the low-burden' cluster, 5) 'the emotionally dysfunctioning' cluster, 6) 'the overall worst-functioning' cluster and 7) 'the physically dysfunctioning' cluster. Again, the conclusion is drawn that these clusters could not replace a goal-directed, personalized management approach (see Chapter 7). ${ }^{16}$

Our data clearly illustrate that even the most complex "control panel" as described in Chapter $7^{16}$ does not contribute to the identification of actionable subgroups of COPD patients referred for PR. It is important to realize that such temporary assessment of traits does not necessarily reflect the level of homeostasis experienced by the patient. ${ }^{17}$ The data described in Chapter $7^{16}$ again underscore that current recommendations to assess and grade COPD on spirometry, dyspnea, and/or health status and exacerbations and/or hospitalizations in the last 12 months $^{2}$ largely underestimate the possible needs in these frail, chronic patients.

\section{Towards individual goals and values?}

As described in Chapter 1, the first ACCP (American College of Chest Physicians) and ATS (American Thoracic Society) statement on pulmonary rehabilitation formulated that the program has to attempt to return the individual patient to the highest possible capacity permitted by the pulmonary handicap and overall life situation ${ }^{18}$ or restore the individual to the fullest medical, mental, emotional, social and vocational potential of which the patient is capable. ${ }^{19}$ Others confirmed that pulmonary rehabilitation must be individually tailored and designed to optimize physical and social performance and autonomy. ${ }^{20}$ From this perspective, outcomes of pulmonary rehabilitation should be defined to changes in the somatic, physical, psychological and social condition that reflect favorable or adverse effects on the patient's well-being. In contrast and likely driven by skepticism on pulmonary rehabilitation, the European Respiratory Society (ERS) restricted outcomes to clinically and physiologically relevant outcome measures. ${ }^{21}$ Later on, improvement in the physical and psychological condition of individuals with a chronic respiratory disease was advocated..$^{22}$ 
In practice, outcomes of pulmonary rehabilitation are largely restricted to reduction of symptoms, improvements in walk distance and health status. ${ }^{23}$ According to the latest GOLD guidelines, patients with high symptom burden and risk of exacerbations are indicated for pulmonary rehabilitation. ${ }^{2}$ Given the profile of a patient with COPD referred for pulmonary rehabilitation, outcome measures need to go beyond the standard health-related quality of life or/and exercise capacity. ${ }^{24}$ Outcomes that really matter to patients with complex needs should take into account the illness of the patient: how a person feels to be ill or experiences a loss of his/her health (illness). ${ }^{25,26}$ Furthermore, the ability to adapt and self-manage in the face of the social, physical and emotional challenges of their life must be considered as outcomes for pulmonary rehabilitation. ${ }^{27,28}$

The impact of such a multidimensional outcome measure existing of eight outcome measurements is summarized in Chapter $5^{29}$ : response to pulmonary rehabilitation was not restricted to functional performance (6-minute walk distance, cycle endurance time or symptoms of dyspnea and health status); anxiety and depression as well as performance and satisfaction with the performance of problematic activities in daily life were also measured. Moreover, patients could be clustered into four groups with distinct multidimensional response profiles: very good, good, moderate and poor responders (see Chapter 5 ). ${ }^{29}$ It is important to note that in contrast to current evidence based thinking, in the very-good responding cluster, the minimal clinically relevant improvement was achieved in $85 \%$ of the outcomes at least once and in $67 \%$ of the outcomes at least twice, indicating that these patients truly benefit from pulmonary rehabilitation. Furthermore, even in the poor-responding group, clinically relevant improvements could be identified in $11 \%$ of the multidimensional outcome panel. In particular, patients in this group still have a significantly better satisfaction score for the problematic activities of daily life, reflecting a better resilience potential after pulmonary rehabilitation. Comparing baseline characteristics in Chapter $5^{29}$, these clusters could not be differentiated based on physiological parameters only: patients in the very-good responders group even seem to have the highest disease burden. Chapter $6^{30}$ confirms the limitations of lung function testing only to predict pulmonary rehabilitation outcomes: no relationship is found between the seven identified lung-function-based clusters and response to pulmonary rehabilitation. Furthermore, predictability of assessment is not improved by including more attributes (see Chapter 8): this study confirms that the very good responders demonstrate the highest disease burden, as manifested by low exercise performance and worse scores on activities in daily living, anxiety, depression and quality of life despite better pulmonary traits. The study in Chapter 8 also confirms the findings of Chapter $5^{29}$ that the number of very-good and good responders is remarkably higher after an inpatient pulmonary rehabilitation program. These findings emphasize not only the need for the integration of psychosocial factors and behavior change in the care of people with chronic respiratory diseases ${ }^{29,31}$, 
but also the challenge of identifying the right patient for the right outcome at the right time. $^{32}$

In the last decade, there has been increased attention on value-based health care (i.e., focusing on maximizing the value of care for patients). Value is defined as the health outcomes achieved divided by costs spent. ${ }^{33}$ In his landmark paper, Porter stated that value should always be defined around the customer and that creation of value should determine the rewards for all actors in the health care system. Outcomes, the numerator in the value equation, must be inherently condition-specific and multidimensional. ${ }^{33}$ Measuring, reporting and comparing outcomes are therefore crucial steps. Our data on outcomes clearly illustrate the need for a multidimensional outcome evaluation in pulmonary rehabilitation. Strikingly, an intervention strategy that claims individualization based on the assessment of individual needs still embraces treatment effects, largely derived from pharmacological clinical trials. Lack of a multidimensional outcome or reliance on a limited set of average effects largely impairs estimation of the real value of pulmonary rehabilitation, and such lack of performance metrics also hampers benchmarking of different programs or pulmonary rehabilitation settings. Such a lack of clarity concerning goals is dangerous and self-defeating, making the intervention prone to false savings under the "cost savings" umbrella. 


\section{References}

1. Han MK, et al. Chronic obstructive pulmonary disease phenotypes: the future of COPD. Am J Respir Crit Care Med. 2010;182(5):598-604.

2. Global Initiative for Chronic Obstructive Lung Disease (GOLD). Global Strategy for Prevention, Diagnosis and management of COPD. 2021.

3. Lange $P$, et al. Diagnosis, assessment, and phenotyping of COPD: beyond FEV(1). Int J Chron Obstruct Pulmon Dis. 2016;11 Spec Iss:3-12.

4. Augustin IML, et al. Understanding Complexity of Chronic Non-Communicable Diseases: An Integrated Approach for Personalized Management of Patients with COPD. Clin Res Pulmonol. 3(2), 2015.

5. Freimer N, Sabatti C. The human phenome project. Nat Genet. 2003;34(1):15-21.

6. Le Rouzic O, et al. Defining the "Frequent Exacerbator" Phenotype in COPD: A Hypothesis-Free Approach. Chest. 2018;153(5):1106-15.

7. Park J, et al. Subtyping COPD by Using Visual and Quantitative CT Imaging Features. Chest. 2020;157(1): 47-60.

8. Agusti A, et al. Treatable traits: toward precision medicine of chronic airway diseases. Eur Respir J. 2016; 47(2):410-9.

9. Erikainen S, Chan S. Contested futures: envisioning "Personalized," "Stratified," and "Precision" medicine. New Genet Soc. 2019;38(3):308-30.

10. FORUM Academy of Medical Science. Stratified, Personalised or P4 Medicine: a New Direction for Placing the Patient at the Centre of Healthcare and Health Education. https://acmedsci.ac.uk/filedownload/38266-56e6d483e1d21.pdf. 2015 [cited 2020 November].

11. Agusti A. Phenotypes and disease characterization in chronic obstructive pulmonary disease. Toward the extinction of phenotypes? Ann Am Thorac Soc. 2013;10 Suppl:S125-30.

12. Agusti A, MacNee W. The COPD control panel: towards personalised medicine in COPD. Thorax. 2013; 68(7):687-90.

13. McDonald VM, et al. Treatable traits: a new paradigm for 21st century management of chronic airway diseases: Treatable Traits Down Under International Workshop report. Eur Respir J. 2019;53(5).

14. Spruit MA, et al. Integration of pulmonary rehabilitation in COPD. Lancet. 2008;371(9606):12-3.

15. Augustin $\mathrm{IML}$, et al. The respiratory physiome: Clustering based on a comprehensive lung function assessment in patients with COPD. PLoS One. 2018;13(9):e0201593.

16. Augustin IML, et al. Incorporating Comprehensive Assessment Parameters to Better Characterize and Plan Rehabilitation for Persons with Chronic Obstructive Pulmonary Disease. J Am Med Dir Assoc. 2020.

17. Ashby RW. An introduction to Cybernetics. 1956: London: Methuen.

18. Hodgkin JE, et al. American Thoracic Society. Medical Section of the American Lung Association. Pulmonary rehabilitation. Am Rev Respir Dis. 1981;124(5):663-6.

19. British Thoracic Society (BTS). BTS guidelines for the management of chronic obstructive pulmonary disease. The COPD Guidelines Group of the Standards of Care Committee of the BTS. Thorax. 1997;52 Suppl 5:S1-28.

20. American Association for Respiratory Care (AARC). AARC Clinical Practice Guideline Pulmonary Rehabilitation. https://www.aarc.org/wp-content/uploads/2014/08/05.02.617.pdf. 2002 [cited 2021 ].

21. Donner CF, Muir JF. Selection criteria and programmes for pulmonary rehabilitation in COPD patients. Rehabilitation and Chronic Care Scientific Group of the European Respiratory Society. Eur Respir J. 1997; 10(3):744-57.

22. Spruit MA, et al. An official American Thoracic Society/European Respiratory Society statement: key concepts and advances in pulmonary rehabilitation. Am J Respir Crit Care Med. 2013;188(8):e13-64.

23. Spruit MA, et al. Differences in content and organisational aspects of pulmonary rehabilitation programmes. Eur Respir J. 2014;43(5):1326-37.

24. Houben-Wilke $S$, et al. COPD stands for complex obstructive pulmonary disease. Eur Respir Rev. 2018; 27(148).

25. Solomon M, Simon JR, Kincaid H. The Routledge Companion to Philosophy of Medicine, chapter 2 "Disease, illness, and sickness". 2017, New York: Taylor \& Francis.

26. Seidlein AH, Salloch S. Illness and disease: an empirical-ethical viewpoint. BMC Med Ethics. 2019;20(1):5.

27. Huber M, et al. How should we define health? BMJ, 2011;343:d4163. 
28. Huber M, et al. Towards a 'patient-centred' operationalisation of the new dynamic concept of health: a mixed methods study. BMJ Open. 2016;6(1):e010091.

29. Spruit MA, et al. Differential response to pulmonary rehabilitation in COPD: multidimensional profiling. Eur Respir J. 2015;46(6):1625-35.

30. Augustin IML, et al. Comprehensive Lung Function Assessment Does not Allow to Infer Response to Pulmonary Rehabilitation in Patients with COPD. J Clin Med 2018;8(1).

31. von Leupoldt A, et al. ERS Scientific Working Group 09.04, "Psychologists and behavioural scientists": the next step towards multidisciplinary respiratory care. Eur Respir J. 2020;56(3).

32. Scholz N. Personalised medicine: The right treatment for the right person at the right time. 2015.

33. Porter ME. What is value in health care? N Engl J Med. 2010;363(26):2477-81. 



\section{Chapter 10}

Future perspectives

"Part of publication "The patient with a complex chronic respiratory disease: a specialist of his own life?" Sarah Houben-Wilke, Ingrid ML Augustin, Birgit BREF Wouters, Rosita AH Stevens, Daisy JA Janssen, Martijn A Spruit, Lowie EGW Vanfleteren, Frits ME Franssen, Emiel FM Wouters Expert Review of Respiratory Medicine. 2017;11(12):919-924. 



\section{"The patient as specialist of his own life or director of his own life?}

All definitions on pulmonary rehabilitation formulate that the primary focus must be the patient and their caregivers. Although considered crucial, most pulmonary rehabilitation programs still lack clear involvement of the patient and carer in supporting them to make effective choices about their care. ${ }^{1}$ Searching through the worldwide web, patient has been defined as "a person who is receiving medical care, or who is cared for by a particular doctor" 2 or "a person or thing that undergoes some action" ${ }^{3}$, demonstrating a more static and dependent role of the patient in the health care system. Indeed, synonyms for "patient" like "case, subject or sufferer" 4 suggest a passive health care seeker. On the other hand, patients are expected to pick up the role of experts, taking responsibility for the day-to-day decisions about their health, and to work with health care providers as collaborators and partners to produce the best possible health given the resources at hand. Expert patients, especially those with chronic disorders, have been said to be not only consumers of health care but also "producers of health". ${ }^{5}$ This must be in particular the case for those participating to pulmonary rehabilitation. The term "expert patient" has already been introduced relating to an initiative to help dealing with chronic illness. ${ }^{6}$ Such expert patient programs "developing the confidence and motivation of patients to use their own skills and knowledge to take effective control over life with a chronic illness" ${ }^{\prime 7}$ have been shown to be effective in decreasing symptoms, improving health behaviors, self-efficacy, and satisfaction with the health care system as well as reducing health care utilization. ${ }^{8}$ The role of the patient extends beyond coping with the disease: patients need to change and adapt their lifestyles and lives underlining the need for a holistic approach. For instance, breathlessness not only impacts the daily life of the patients but also the daily lives of their social environment, for example, family caregivers. ${ }^{9}$ Furthermore the responsibility of patients as specialists of their own lives is to express what's important in their lives and thereby, in collaboration with health care providers, to specify relevant treatment goals helping them to live the lives they want to.

In order to achieve and maintain the maximal level of independence and functioning of the patient within the community, self-management is widely considered as a cornerstone. Self-management refers to "the ability of the individual, in conjunction with family, community, and health care professionals, to manage symptoms, treatments, lifestyle changes, and psychosocial, cultural, and spiritual consequences of health conditions". ${ }^{10}$ Self-management might be facilitated by interactive counselling approaches actively involving patients in their disease management. ${ }^{11}$ Self-management interventions have been shown to be associated with improved health status, reduced hospital admissions, and less symptoms of dyspnea. ${ }^{12}$ 
In order to act as a specialist of his own life, the patient is expected to play its role in shared decision-making, an important component of patient-centered care. Shared decision-making has been defined as "an approach where clinicians and patients share the best available evidence when faced with the task of making decisions, and where patients are supported to consider options, to achieve informed preferences". ${ }^{13}$ Thus, shared decision-making does not mean that the patient has to make the final decision; it does mean that the health care professional needs information from the patient about his beliefs and expectations about certain treatment options as well as outcomes, and explains advantages as well as disadvantages helping the patient to be able to specify what is important for him. Since it is hardly possible to predict patients' treatment preferences ${ }^{14}$ and since a disparity exists between health care professionals' judgments and patients' preferences (which may further reflect ineffective communication), incorporating the patient into treatment decisions is clearly needed. ${ }^{15}$ Patients reporting good communication with their doctor are more satisfied with their care, and are more likely to follow advice and adhere to treatment. Furthermore, good doctor - patient communication has been shown to be associated with regulating patients' emotions, facilitating comprehension of medical information as well as better identification of patients' needs and expectations. ${ }^{16}$ Thus, we need to release the traditional idea of "the doctor tells me what to do"; the patients' role changes from being "users and choosers" to becoming "makers and shapers" of health care services. ${ }^{17}$

In order to play this role of makers or shapers, patients must have excellent communication skills. Therefore, patients - as specialists of their own lives - are encouraged to be conscious and to express their feelings, perceptions, and symptoms and to be involved in the process of active information seeking. They should further be able to discuss what's important in life, to specify their desirable way of living, their sense of life and their meaning of quality of life which should be fundamental for defining successful, individualized treatment goals meeting patients' expectations. Patients must also be able to identify exacerbations based on visible as well as invisible symptoms and able to apply self-management strategies to manage their disease facilitating early awareness and treatment of exacerbations. ${ }^{18}$

Is this a realistic scenario? Can we expect all these skills from our patients? The answer is probably negative under the current circumstances. This is in particular the case for the high-needs patients as referred for pulmonary rehabilitation. First at all, cognitive impairment $^{19}$ and poor health literacy ${ }^{20,21}$ are prevalent in these patients with COPD which have further been shown to be associated with worse clinical outcomes, health status, illness beliefs that predicted decreased adherence. ${ }^{20,21}$ Moreover, various coping styles exist in patients with COPD $^{22}$ demonstrating different competences and capabilities. Only a minority of COPD patients entering a pulmonary rehabilitation program uses a high or very high level of active confronting coping style. ${ }^{23}$ Intriguingly, comprehensive pulmonary rehabilitation results in change in coping style of these COPD 
patients and coping styles are related to improvements in exercise tolerance, anxiety and depression. ${ }^{22}$ Poor coping is further suggested by maladaptive behaviors such as non-adherence to lifestyle recommendations, missing medical appointments, not taking prescribed medication or avoiding laboratory testing. ${ }^{24}$ But also health care providers are not the best communicators, and care and treatments are highly fragmented: health care providers are still working in silos and are frequently not willing to support holistic care needs. ${ }^{1}$ In the todays' paternalistic health care system, health care providers are still the medical decision makers. ${ }^{25}$ As a consequence, decision-making mainly concentrates on disease-specific outcomes following practice guidelines for specific conditions. ${ }^{26}$ Multiple subspecialty consultants, each focused only on their chosen organ system, tend to create incoherent and burdensome treatment plans aimed at various outcomes that may or may not be at all important to the patient. ${ }^{27}$ As an example, treating a patient with comorbidities for each disease independently often results in polypharmacy along with the subsequent adverse and cross-reactions. Besides the use of multiple respiratory medications in symptomatic patients with mild to very severe COPD referred for pulmonary rehabilitation ${ }^{28}$, complex drug regimens arise in those COPD patients with multiple comorbidities. ${ }^{29}$ Indeed, multiple adherence to guidelines for individual chronic diseases might lead to potential cumulative side-effects, interactions, and difficulties with compliance and in such situations, it might be challenging for clinicians to optimize treatment in individuals. ${ }^{29}$ Therefore, patient and health care professionals are not equivalent partners since patients are forced into a more dependent role. ${ }^{30}$ Studies also demonstrate the importance of addressing health literacy in targeted treatment strategies. ${ }^{20,21}$ For instance, patients identified difficult medical language as barriers for effective communication ${ }^{31,32}$, encouraging health care professionals to pay attention to their diction and the exchange of information. It is important to provide time for the patient to think about information or news. ${ }^{31}$ In addition, family support, peer support, visual teaching material, and a trusting empathic patient - provider relationship might improve communication of medical information. ${ }^{32}$ Patients experiencing barriers or lack of knowledge must be further stimulated to explicitly specify that they have any further questions. They must be encouraged to be prepared for a medical consult by eventually using supportive tools (e.g. information material suggesting relevant questions, etc.). Finally, it is important to clarify that no one expects medical but personal expertise from the patient: experiences and know-how from the particular patient about the patient's own life, values and preferences. ${ }^{31}$ Future pulmonary rehabilitation, putting the patient in the core of the program, have to start by clear clarification of the role of the patient.

\section{From traits to needs}

The current trait dominant strategy relates all measurable changes to the disease condition itself. This approach largely relies on the 1948 definition of health defined as a 
state of complete physical, mental and social well-being. ${ }^{33}$ Furthermore, the interaction of different traits is completely overlooked. The human being is able to maintain an internal equilibrium in the face of external perturbations as disease conditions. ${ }^{34}$ This mechanism is called homeostasis and the patient can maintain homeostasis across a wide range of environmental changes. Therefore, from a patient perspective, it would become more appropriate to identify disturbances in homeostasis or needs experienced by the patient itself. Such approach also fits with the current definition of health, emphasizing the ability to adapt and self-manage in the face of social, physical and emotional challenges. ${ }^{35}$ From the beginning of pulmonary rehabilitation, such approach is formulated in the first authoritative statement and reformulated in all definitions of pulmonary rehabilitation. ${ }^{36}$

Such high needs approach is not restricted to COPD alone. Recently, an actionable patient taxonomy is developed to understand and manage these high-need patients. ${ }^{37}$ Six segments are assigned ${ }^{38,39}$ and the National Academy of Medicine recently published a document to explore opportunities for improving outcomes, values and health for these cohorts. ${ }^{40}$ In this taxonomy (see Figure 10.1), besides COPD, following eight conditions are defined as "complex": acute myocardial infarction/ischemic heart disease, chronic kidney disease, congestive heart failure, dementia, psychiatric disease, specified heart arrhythmias, stroke and diabetes. ${ }^{38}$ High needs is largely the driver of high direct medical costs. Considering the assessment characteristics described in Chapter $7^{41}$, all patients referred for pulmonary rehabilitation in our current health care setting fulfil these criteria of high needs, high costs patients. Clearly, needs in all these patients as well as in our cohort extend largely beyond care for physical or medical ailments to behavioral and social aspects. Addressing clinical needs only will partly improve outcomes in these patients: the document underscores the importance to address the functional, behavioral and social needs. ${ }^{40}$ This approach fits not only with the current health concept but also with the concept of personomics. ${ }^{35,42-44}$ Understanding of the relevant psychological, social, cultural, behavioral and economic factors not only determine how a disease reveals itself but also the way how an individual with a disease respond to treatment. 


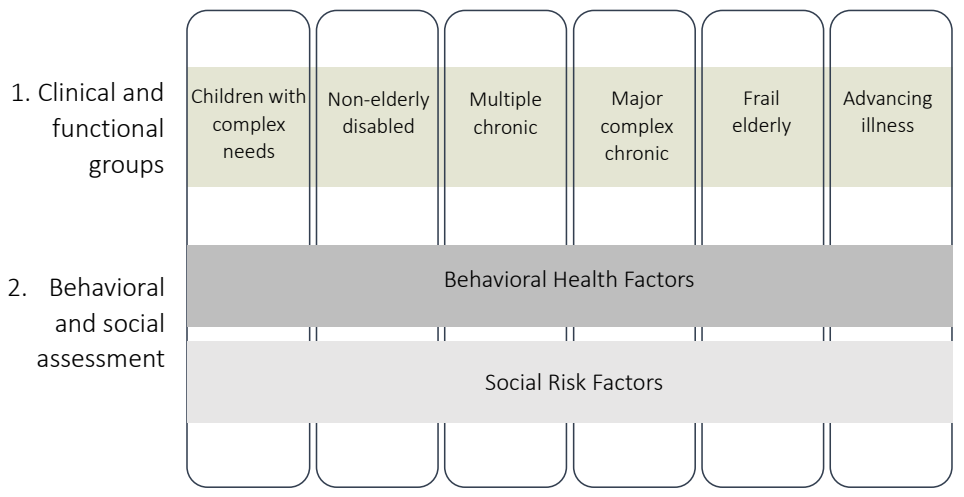

Figure 10.1 A conceptual model of a starter taxonomy for high-need patients. This Figure is reused with permission. ${ }^{40}$

This is particular true for patients with COPD and their informal caregivers, confronted daily with multiple limitations due to COPD. ${ }^{45}$ Indeed, patient's home environment plays an important role in the process of disease management emphasizing the need to include it as a relevant part for treatment strategies. ${ }^{45}$ Kosteli et al. examined the factors that both encourage and limit participation in physical activity for individuals with COPD and found that the main barriers were not only health related (fatigue, mobility problems, breathing issues caused by the weather), but also psychological (embarrassment, fear, frustration/disappointment), attitudinal (feeling in control of their condition, physical activity perception, older age perception), and motivational. ${ }^{46}$ In order to enable response to the individual patient's needs, it is for example important to understand patient-specific social cognitive influences on physical activity participation. ${ }^{46}$ Another study demonstrated that structural social support (e.g. living status, having a partner, having a caregiver) is associated with higher levels of physical activity and greater participation in pulmonary rehabilitation in patients with COPD, while higher functional social support (e.g. emotional and informational, tangible, affectionate, positive social interaction) was associated with, for instance, lower odds of smoking. ${ }^{47}$ Severely impaired quality of life, irrespective of lung function, is considered as a reason to explore positive benefits of psychological and behavioral support for distressed COPD patients. ${ }^{48}$ In order to really improve health outcomes, interventions should not be investigated isolated from the environment, but should focus on the interaction between a person and the environment. A person's goals, hopes, fears, barriers to care, psychological state, and financial and family resources always must be considered in designing effective treatments. ${ }^{43}$ As behavioral change is incorporated in the latest definition of pulmonary rehabilitation ${ }^{49}$, it will be important to analyze and focus on key determinants of health behaviors, to develop more effective interventions to change health behaviors and to link behavior changes to the overall goals of patient value based care. ${ }^{50}$ 


\section{Revisiting management programs to address patient's needs}

The management of patients with COPD is challenging: besides multidimensional patient assessment, alignment between patient goals and functional needs, patient and care partner engagement, communication is a crucial care attribute. ${ }^{40} \mathrm{~A}$ patient-centered approach fosters interactions in which clinicians and patients engage in two-way sharing of information; explore patients' values and preferences; help patients and their families make clinical decisions; facilitate access to appropriate care; and enable patients to follow through with often difficult behavioral changes needed to maintain or improve health. ${ }^{51}$

Health care professionals come to know and respect patients' values, beliefs, hopes, and ways of dealing with adversity, in addition to patients' clinical symptoms and concerns. Clinicians should know the patient's family circumstances and cultural norms well enough to help him or her with decisions about care, adherence to treatment regimens, and self-management to promote healing and prevent illness. ${ }^{51}$ In this context, clinicians can work to provide personalized care that is attuned to the needs of a particular patient. From that perspective, the World Health Organization (WHO) advocates partnership in care. ${ }^{52,53}$ The WHO defines partnership as a collaborative relationship between two or more parties based on a trust, equality and mutual understanding for the achievement of a specific goal. Such partnership involves risks as well as benefits, making shared accountability critical. Conditions for such partnership are absolute interdependence, mutual accountability, collaborative relationship, equal participation in decision making and shared power. ${ }^{52,53}$ An important aspect in this approach is emotional intelligence, i.e. the capacity to understand and manage personal thoughts and feelings, as well as to positively influence interpersonal communication and social well-being, which has been shown to be associated with self-management abilities and health status. ${ }^{54}$

This premise of different levels and dimensions of a clinical problem implies not only the collaboration of different medical disciplines but also a holistic approach of patients beyond the fragmented vision of each specialty. Transdisciplinarity represents the next level in this holistic approach. In the past, multidisciplinarity or interdisciplinarity were frequently integrated in pulmonary rehabilitation definitions. The more general term "multiple-disciplinary" is used when the nature of involvement of multiple disciplines is unknown or unspecified. Interdisciplinarity analyses, synthetizes and harmonizes links between disciplines into a coordinated and coherent whole. Transdisciplinarity transcends the traditional boundaries and upgrades the standards of health care far beyond the unique vision of each discipline. ${ }^{55}$ Transdisciplinarity aims a different way of thinking, and a different way of organizing knowledge. ${ }^{56}$ Morin described this organizing of knowledge as "thinking about thinking": the question is not just what we know, but how we know, and how we organize our knowledge. ${ }^{56}$ In clinical practice, persons joining 
transdisciplinary teams must learn about the other disciplines at least at a level where they can understand the concepts and principles and how it all fits in the work. Assembling a transdisciplinary team, each member will speak the "language" derived through his or her training. ${ }^{57}$

As transdisciplinary organizations are complex adaptive systems bringing together actors from varied backgrounds, promoting interdependence among them, and form dynamic collectives with common goals ${ }^{58}$, traditional leadership and management approaches are no longer sufficient to deal with the organizational and contextual complexity. Complexity leadership theory perceives leadership as an interplay between administrative, enabling and adaptive leadership. ${ }^{59}$ The first, administrative leadership, resembles the traditional, bureaucratic, and hierarchical type of leadership. The second, enabling leadership, operates between administrative and adaptive leadership adopting behaviors for enhancing interactive and adaptive dynamics and monitoring the organization to better understand the different forces influencing the emerging adaptive dynamics. The third, adaptive leadership, is a complex dynamic rather than a role assigned to a person. It is an interactive type of leadership that underlies emergent change activities. ${ }^{59}$

In Chapter 3 pulmonary rehabilitation was described as a holistic approach by considering patients as complex adaptive systems. ${ }^{60}$ At that time a modular program structure integrating the identification of traits as included in this thesis was proposed allowing clustering and evaluating pulmonary rehabilitation by multidimensional response. The redesigning of the structure was based on sociotechnical principles ${ }^{61}$ establishing a relationship between design and quality of the organization, quality of labour relations and quality of working life. Although sociotechnical principles are intended to support the design of sociotechnical systems that meet open systems principles and an integrated approach ${ }^{62}$, the heterogeneity and variability within and between the traits-based clusters found in the previous chapters (Chapter 4, 5, 6, 7 and $8)^{41,63-65}$ provides new perspectives. First, from the perspective of the patients with highneeds and as also earlier described ${ }^{66}$, in the ability to adapt and self-manage, future research needs to identify the optimal set of measures to assess the stimuli related to social, physical and psychological functioning in patients with COPD. Ashby, in 1956, already explained the challenge of an adaptive system, i.e. a system survives to the extent that the range of responses it is able to marshal successfully matches the ranges of situations confronted it. ${ }^{34}$ Figure 10.2 demonstrates the Ashby's law in three regimes: the ordered, complex and chaotic regime with the diagonal indicating the set of points at which variety of a system's response matches that of incoming stimuli in an adaptive way - it facilitates survival whether or not it does so with an efficient use of resources a system must possess complexity equal to that of its environment in order to function effectively. ${ }^{67}$ As this model positions complexity and organization-environment relations, a system must possess complexity equal to that of its environment in order to function 
effectively ${ }^{67}$, a complex adaptive system as a collection of individual agents with freedom to act in ways that are not always predictable, and whose actions are interconnected, is also seen in a biochemical system or on human being expressed as instincts, constructs, and mental models. ${ }^{68}$ Applying this model for patients with multidimensional problems (on the left side of Figure 10.2), it would become important to identify those stimuli in the functional, emotional and social domains corroborating to disproportionate responses. High-needs patients are patients demonstrating disproportionate reactions in the chaotic domain according to Ashby's model, i.e. homeostatic imbalance. The pulmonary rehabilitation program must focus on a behavioral intervention in order to bring the patient back to an equilibrium. This means that our patients needs first to interpret the stimuli impinging upon it in order to generate more adaptive responses depending on its cognitive limits and its capacity to process the variety of stimuli. The optimal outcome will be when the patient will develop a routeing response: in this case, all incoming stimuli will be treated either as familiar regularities or as noise not needing any new response. From an organizational perspective, the current traits based approach, ignoring this stimulus-response relationship will ultimately end in an chaotic regime depleting budget resources.
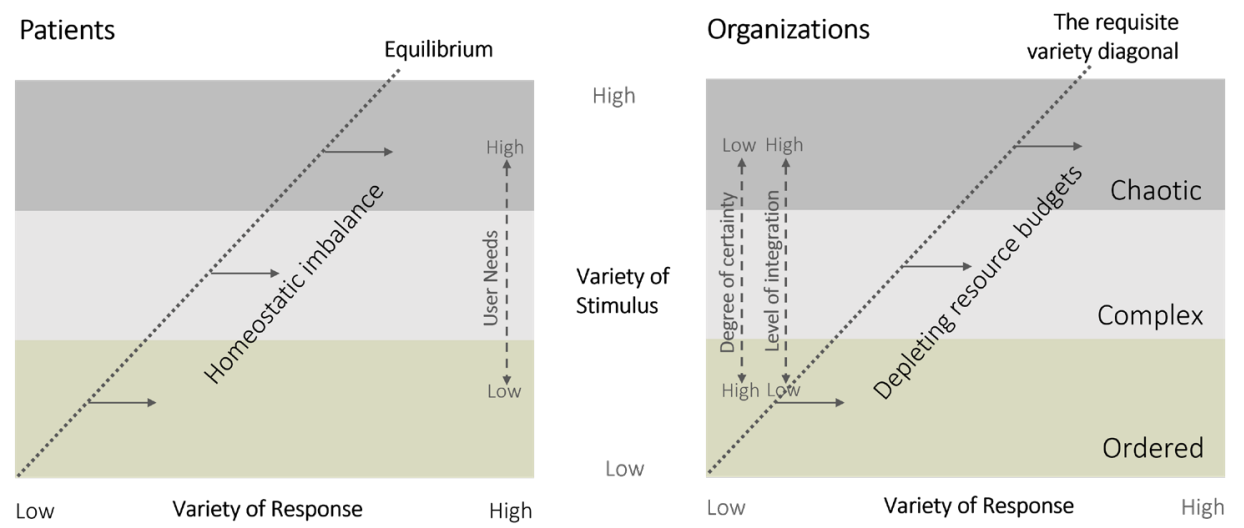

Figure 10.2 Ashby's law in three regimes.

Since unpredictability and uncertainty is high in the chaotic regime ${ }^{69}$, a transdisciplinary approach as outlined above with a high level of integration will be appropriate. ${ }^{70}$ As described in Chapter 3, complex interventions should be centralized and organized as an integrated and coordinated practice unit. ${ }^{71,72}$ In this structure, all attributes as pointed out above should be integrated in the so-called high-value health care organizations that are featured by separation of heterogenous patients into clinically meaningful subgroups, allocating various tasks of care to different members of a clinical team (including the patient as member of the team), collecting more detailed measurements than those required (regarding quality and efficiency) and ongoing seeking of new 
insights and better outcomes for their patients (creating knowledge and innovation) (see also chapter 3).60,72 Discussions about the best care for high-complex patients will continue, illustrating that the design of a program depends on policy of its country. Whatever the care-model chosen, both patient-centeredness and personalized medicine are required in order to deliver high value care for patients with complex high needs and rely importantly on the competencies of the team.

\section{From last resort to an integrated outreach program}

Effective, value based care models for high-need patients as those referred for pulmonary rehabilitation also requires supporting and rewarding the seamless integration of these medical, behavior and social services as well as support for delivery of these services in the home and community setting: outreach of care is still a neglected aspect in the effective management of these patients. ${ }^{40}$ Indeed, the current health care systems for patients with high-needs are highly fragmented during the journey of patients with chronic conditions. Already two decades ago, a process based definition describes integrated care as a coherent set of methods and models on the funding, administrative, organizational, service delivery and clinical levels designed to create connectivity, alignment and collaboration between the cure and care sectors to enhance quality of care and quality of life, consumer satisfaction and system efficiency for patients with complex problems cutting across multiple services, providers and settings. ${ }^{73}$ What will become important is a patient's definition of integrated care: "I can plan my care with people who work together to understand me and my carer, allow me control, and bring together services to achieve the outcomes important to me". ${ }^{74}$ Indeed, we must build a network to support the patient and the carer and not a labyrinth to get them lost. Otherwise, we will do these high-needs and very vulnerable patients more harm than good. As described above, a high level of user need requires a high level of integration including a high level of different service domains for patients with high needs. ${ }^{70}$ In a Dutch report, an $A B C D$ model including prevention $(A)$, community care (B), low to complex care and (C) and high-complex care (D), has been introduced with high-complex care described as a complex intervention with a low degree of predictability of the required quantitative and qualitative input leading to an ongoing adjusting of treatment based on further diagnostics and observation. ${ }^{75}$ In line with the National Academy of Medicine ${ }^{37}$, the focus of high-complex care is on patients with high-complex problems (with one or more chronic disease(s) and/or problems with activities in daily life and/or psychological and/or social problems) and is performed in a specialized environment by a dedicated team existing of multiple disciplines. ${ }^{75}$ The European framework for Action on Integrated Health Services Delivery correctly stated strategizing with people at the center of the framework: identifying health needs, tackling determinants, empowering populations and engaging patients. ${ }^{76}$ 
Both personalized medicine and patient-centered care are important in creating high value for these complex patients with high needs. Personalized medicine as the concept of better understanding the complexity of the disease based on stratifying patients, might aspire more control over the optimization of medical treatment results, more precision, increasing predictability of disease, and thus control over the outcomes. A patient-centered care model builds on the person's agency and empowerment, and may thus involve increased uncertainty of the outcome and less control for professionals. ${ }^{77}$ Building on the person's agency and empowerment, Sullivan described agency as the capacity for action; specifically, the capacity to do or be something in accord with one's values $^{78}$, Actually, the capacity of the patient to be a specialist of his own life. ${ }^{79}$ This ultimate overall goal goes beyond the individual components of patient-centered care, i.e. enhancing self-management, empowering autonomy and participation, shareddecision making and enhancing health behavior ${ }^{79}$ : behavior remains the scientifically favored term to describe the acting and doing of patients. ${ }^{78}$ Healthy patients are not just those who emit healthy behaviors, but patients who are agents in their own lives. ${ }^{78}$ Engelhardt et al. argued that there is not one but many possible accounts of patient autonomy. ${ }^{80}$ It cannot simply be a content-free means of choosing the good life: autonomy implies "strikingly different understanding of appropriate behavior" in multiple controversial areas. ${ }^{80}$ The patient's moral agency or decisional autonomy does not stand apart from his illness. ${ }^{78}$ Patients with complex chronic diseases with high needs have impaired autonomy in the broadest sense and are less able to formulate and pursue life plans. Their ability to initiate and complete meaningful actions of all sorts is impaired. ${ }^{78}$ Shared decision making is an important component of patient-centered care and can only take place within an ongoing partnership between clinician and patient, both respecting the other as a person, not as part of an isolated encounter. ${ }^{81}$ Respect for the patient as a person goes beyond respect for their choice. ${ }^{81}$ Knowing the patient as a person and providing an autonomy-supportive context for care are crucial. ${ }^{81}$

In this context, pulmonary rehabilitation creates an environment to offer the patient a platform to reset the level of homeostasis to adapt and manage the external complexity. Therefore, pulmonary rehabilitation as an individualized, comprehensive intervention will form a crucial step towards better health for COPD patients in particular and to high needs patients in general. 


\section{References}

1. Meyer I. Achieving Effective Integrated E-Care Beyond the Silos. 2014.

2. What is patient. http://www.igi-global.com/dictionary/patient/22039. [cited 2021 ].

3. Patient. http://www.dictionary.com/browse/patient. [cited 2021].

4. Synomyms for "patient". http://www.thesaurus.com/browse/patient?s=t. [cited 2021 ].

5. Lorig K. Partnerships between expert patients and physicians. Lancet. 2002;359(9309):814-5.

6. Health Do. Saving lives: our healthier nation. The Stationery Office, London, 1999.

7. Health Do. The expert patient: a new approach to chronic disease management in the 21st century. Department of Health, London, 2001.

8. Lorig KR, et al. The Expert Patients Programme online, a 1-year study of an Internet-based selfmanagement programme for people with long-term conditions. Chronic IIIn. 2008;4(4):247-56.

9. Janssen DJ, Wouters EF, Spruit MA. Psychosocial consequences of living with breathlessness due to advanced disease. Curr Opin Support Palliat Care. 2015;9(3):232-7.

10. Richard AA, Shea K. Delineation of self-care and associated concepts. J Nurs Scholarsh. 2011;43(3): 255-64.

11. Singh SJ, et al. Learn from the past and create the future: the 2013 ATS/ERS statement on pulmonary rehabilitation. Eur Respir J. 2013;42(5):1169-74.

12. Zwerink M, et al. Self management for patients with chronic obstructive pulmonary disease. Cochrane Database Syst Rev. 2014;(3):CD002990.

13. Elwyn G, et al. Implementing shared decision making in the NHS. BMJ. 2010;341:c5146.

14. Janssen DJA, et al. Predicting changes in preferences for life-sustaining treatment among patients with advanced chronic organ failure. Chest. 2012;141(5):1251-9.

15. Muhlbacher AC, Juhnke C. Patient preferences versus physicians' judgement: does it make a difference in healthcare decision making? Appl Health Econ Health Policy. 2013;11(3):163-80.

16. Ha JF, Longnecker N. Doctor-patient communication: a review. Ochsner J. 2010;10(1):38-43.

17. Janamian $T$, Crossland L, Wells $L$. On the road to value co-creation in health care: the role of consumers in defining the destination, planning the journey and sharing the drive. Med J Aust. 2016;204(7 Suppl): S12-4.

18. Williams $V$, et al. Patients' experience of identifying and managing exacerbations in COPD: a qualitative study. NPJ Prim Care Respir Med. 2014;24:14062.

19. Cleutjens FA, et al. Domain-specific cognitive impairment in patients with COPD and control subjects. Int J Chron Obstruct Pulmon Dis. 2017;12:1-11.

20. Puente-Maestu L, et al. Health literacy and health outcomes in chronic obstructive pulmonary disease. Respir Med. 2016;115:78-82.

21. Kale MS, et al. The Association of Health Literacy with Illness and Medication Beliefs among Patients with Chronic Obstructive Pulmonary Disease. PLoS One. 2015;10(4):e0123937.

22. Stoilkova A, et al. Coping styles in patients with COPD before and after pulmonary rehabilitation. Respir Med. 2013;107(6):825-33.

23. Stoilkova A, et al. The relationship between coping styles and clinical outcomes in patients with COPD entering pulmonary rehabilitation. COPD. 2013;10(3):316-23.

24. Grady KL, et al. Factors associated with stress and coping at 5 and 10 years after heart transplantation. J Heart Lung Transplant. 2013;32(4):437-46.

25. Rodriguez-Osorio CA, Dominguez-Cherit G. Medical decision making: paternalism versus patientcentered (autonomous) care. Curr Opin Crit Care. 2008;14(6):708-13.

26. Tinetti ME, Naik AD, Dodson JA. Moving From Disease-Centered to Patient Goals-Directed Care for Patients With Multiple Chronic Conditions: Patient Value-Based Care. JAMA Cardiol. 2016;1(1):9-10.

27. May $C R$, et al. Rethinking the patient: using Burden of Treatment Theory to understand the changing dynamics of illness. BMC Health Serv Res. 2014;14:281.

28. Franssen FM, Spruit MA, Wouters EF. Determinants of polypharmacy and compliance with GOLD guidelines in patients with chronic obstructive pulmonary disease. Int J Chron Obstruct Pulmon Dis. 2011;6:493-501.

29. Vanfleteren L, et al. Management of chronic obstructive pulmonary disease beyond the lungs. Lancet Respir Med. 2016;4(11):911-24. 
30. Specialisten FM. Visiedocument "Medisch Specialist 2025". 2017.

31. Pieterse A, Brand P, Basoski N. Alles wat u meten over gedeeld besluitvorming. Medisch Contact. 2017; 23:12:34-36.

32. Sadeghi S, Brooks D, Goldstein RS. Patients' and providers' perceptions of the impact of health literacy on communication in pulmonary rehabilitation. Chron Respir Dis. 2013;10(2):65-76.

33. World Health Organization (WHO). https://www.who.int/publications/m/item/constitution-of-the-worldhealth-organization. 2006.

34. Ashby RW. An introduction to Cybernetics. 1956: London: Methuen.

35. Huber M, et al. How should we define health? BMJ. 2011;343:d4163.

36. Hodgkin JE, et al. American Thoracic Society. Medical Section of the American Lung Association. Pulmonary rehabilitation. Am Rev Respir Dis. 1981;124(5):663-6.

37. National Academy of Medicine. Effective care for high-need patients: opportunities for improving outcomes, value, and health. https://nam.edu/HighNeeds/highNeedPatients.html. 2017.

38. Joynt KE, et al. Segmenting high-cost Medicare patients into potentially actionable cohorts. Healthc (Amst). 2017;5(1-2):62-7.

39. Zhang $\mathrm{Y}$, et al. Developing an actionable patient taxonomy to understand and characterize high-cost Medicare patients. Healthc (Amst). 2020;8(1):100406.

40. Long $P$, et al. Effective Care for High-Need Patients: Opportunities for Improving Outcomes, Value, and Health. Washington DC: National Academy of Medicine. 2017( ).

41. Augustin IML, et al. Incorporating Comprehensive Assessment Parameters to Better Characterize and Plan Rehabilitation for Persons with Chronic Obstructive Pulmonary Disease. J Am Med Dir Assoc. 2020.

42. Huber $\mathrm{M}$, et al. Towards a 'patient-centred' operationalisation of the new dynamic concept of health: a mixed methods study. BMJ Open. 2016;6(1):e010091.

43. Ziegelstein RC. Personomics. JAMA Intern Med. 2015;175(6):888-9.

44. Ziegelstein RC. Personomics: The Missing Link in the Evolution from Precision Medicine to Personalized Medicine. J Pers Med. 2017;7(4).

45. Nakken N, et al. Informal caregivers of patients with COPD: Home Sweet Home? Eur Respir Rev. 2015; 24(137):498-504.

46. Kosteli MC, et al. Barriers and enablers of physical activity engagement for patients with COPD in primary care. Int J Chron Obstruct Pulmon Dis. 2017;12:1019-31.

47. Chen Z, et al. Association between Social Support and Self-Care Behaviors in Adults with Chronic Obstructive Pulmonary Disease. Ann Am Thorac Soc. 2017;14(9):1419-27.

48. Brien SB, Lewith GT, Thomas M. Patient coping strategies in COPD across disease severity and quality of life: a qualitative study. NPJ Prim Care Respir Med. 2016;26:16051.

49. Spruit MA, et al. An official American Thoracic Society/European Respiratory Society statement: key concepts and advances in pulmonary rehabilitation. Am J Respir Crit Care Med. 2013;188(8):e13-64.

50. Conner M, Norman P. Health behaviour: Current issues and challenges. Psychol Health 2017;32(8): 895-906.

51. Epstein RM, et al. Why the nation needs a policy push on patient-centered health care. Health Aff (Millwood). 2010;29(8):1489-95.

52. World Health Organization (WHO). Patient safety. APPS definition of partnership. https://www.who.int/ patientsafety/implementation/apps/definition/en/. [cited 2021].

53. World Health Organization (WHO). Patient safety. APPS definition of partnership. https://www.who.int/ patientsafety/implementation/apps/resources/defining_partnerships-apps.pdf?ua=1. 2009 [cited 2021 ].

54. Benzo RP, et al. Emotional Intelligence: A Novel Outcome Associated with Wellbeing and SelfManagement in Chronic Obstructive Pulmonary Disease. Ann Am Thorac Soc. 2016;13(1):10-6.

55. Alexinschi O. The Need for Transdisciplinarity in Psychiatry and Addiction Medicine. Biomed J Sci \& Tech Res. 2020;25(4).

56. Morin E. On Complexity. 2008, United States of America: Hampton Press, Inc.

57. Di Mattina JW, Pronovost PJ, Holzmueller CG. Transdisciplinary Teams Spur Innovation for Patient Safety and Quality Improvement. Qual Manag Health Care. 2017;26(2):124-5.

58. Lotrecchiano GR. Complexity leadership in transdisciplinary (TD) learning environments: A knowledge feedback loop. International Journal of Transdisciplinary Research. 2010;5:29-6\3.

59. Uhl-Bien M. Complexity Leadership Theory: Shifting leadership from the industrial age to the knowledge era. Leadership Quarterly. 2007. 
60. Augustin IML, et al. Understanding Complexity of Chronic Non-Communicable Diseases: An Integrated Approach for Personalized Management of Patients with COPD. Clin Res Pulmonol. 3(2), 2015.

61. Amelsvoort PJLM van. The design of work and organisation. 2000: Vlijmen : STGroep Vlijmen. 116 p.

62. Clegg, C.W., Sociotechnical principles for system design. Appl Ergon. 2000;31(5):463-77.

63. Augustin $\mathrm{IML}$, et al. The respiratory physiome: Clustering based on a comprehensive lung function assessment in patients with COPD. PLoS One. 2018;13(9):e0201593.

64. Spruit MA, et al. Differential response to pulmonary rehabilitation in COPD: multidimensional profiling. Eur Respir J. 2015;46(6):1625-35.

65. Augustin IML, et al. Comprehensive Lung Function Assessment Does not Allow to Infer Response to Pulmonary Rehabilitation in Patients with COPD. J Clin Med. 2018;8(1).

66. Spruit MA, et al. A new perspective on COPD exacerbations: monitoring impact by measuring physical, psychological and social resilience. Eur Respir J. 2016;47(4):1024-7.

67. Boisot M, McKelvey B. Complexity and Management, Chapter16: Complexity and OrganizationEnvironment Relations: Revisiting Ashby's Law of Requisite Variety, ed. P. Allen, S. Maguire, and B. McKelvey. 2011, Los Angeles London New Delhi Singapore Washington DC: Sage.

68. Plsek PE, Greenhalgh T. Complexity science: The challenge of complexity in health care. BMJ. 2001; 323(7313):625-8.

69. Wilson T, Holt T, Greenhalgh T. Complexity science: complexity and clinical care. BMJ. 2001;323(7314): 685-8.

70. Leutz WN. Five laws for integrating medical and social services: lessons from the United States and the United Kingdom. Milbank Q. 1999;77(1):77-110, iv-v.

71. Porter M, Teisberg E. Redefining health care: value-based competition on resutts. 2006: Boston: Harvard Business School Press.

72. Bohmer RM. The four habits of high-value health care organizations. N Engl J Med. 2011;365(22):2045-7.

73. Kodner DL, Spreeuwenberg C. Integrated care: meaning, logic, applications, and implications--a discussion paper. Int J Integr Care. 2002;2:e12.

74. Lewis RQL, et al. Where next for integrated care organisations in the English NHS? https://www.nuffieldtrust.org.uk/files/2017-01/where-next-integrated-care-english-nhs-web-final.pdf. The Nuffield Trust. 2010, London.

75. Kaljouw M, Van Vliet K. Naar nieuwe zorg en zorgberoepen: de contouren. https://www.zorginstituutnederland.nl/publicaties/adviezen/2015/04/10/naar-nieuwe-zorg-enzorgberoepen-de-contouren, 2015.

76. World Health Organization (WHO). The European Framework for Action on Integrated Health Services Delivery: an overview (2016) https://www.euro.who.int/en/health-topics/Health-systems/healthservices-delivery/publications/2016/the-european-framework-for-action-on-integrated-health-servicesdelivery-an-overview-2016. 2016 [cited 2021].

77. El-Alti L, Sandman L, Munthe C. Person Centered Care and Personalized Medicine: Irreconcilable Opposites or Potential Companions? Health Care Anal. 2019;27(1):45-59.

78. Sullivan MD. The Patient as Agent of Health and Health Care. 2017, United States of America by Oxford University Press: Oxford University Press.

79. Houben-Wilke $S$, et al. The patient with a complex chronic respiratory disease: a specialist of his own life? Expert Rev Respir Med. 2017;11(12):919-24.

80. Engelhardt HT Jr. The many faces of autonomy. Health Care Anal. 2001;9(3):283-97.

81. Tonelli MR, Sullivan MD. Person-centred shared decision making. J Eval Clin Pract. 2019;25(6):1057-62. 

Samenvatting 


\section{Samenvatting}

COPD (chronisch obstructieve longziekte) wordt beschouwd als een complex syndroom met meerdere pulmonale en extra-pulmonale kenmerken. Ondanks groeiend inzicht in de complexiteit van de ziekte, blijven vele factoren die de gezondheidsstatus en ziektelast van de individuele patiënt beïnvloeden, slecht gediagnosticeerd en onbehandeld. Van oudsher is longrevalidatie een geïndividualiseerd behandelprogramma dat zich, na optimale farmacologische behandeling, richt op de aanhoudende fysiopathologische en psychopathologische problemen (hoofdstukken 1 en 3). Dit geïndividualiseerd behandelprogramma is gebaseerd op een uitgebreid en integraal assessment van ziektekenmerken. Echter, het ontbreken van consensus over de verschillende componenten en domeinen van dit assessment levert een belangrijke bijdrage aan de heterogeniteit van de huidige longrevalidatie programma's (hoofdstuk 1).

De heterogeniteit van COPD met betrekking tot klinische presentatie, fysiologie, beeldvorming, respons op therapie, achteruitgang in longfunctie en overleving wordt algemeen erkend in de COPD-literatuur. Om deze heterogeniteit aan te pakken, werd als potentiële oplossing het identificeren en groeperen van kerneigenschappen van het COPD syndroom in klinisch zinvolle en bruikbare subgroepen ten behoeve van gerichte therapie geïntroduceerd. Helaas worden in COPD-onderzoek operationele definities toegepast om deze mogelijke subgroepen of zogenaamde fenotypes te omschrijven. Terwijl een fenotype per definitie de waarneembare structurele en functionele eigenschappen van een organisme weergeeft, bepaald door zijn genotype en gemoduleerd door zijn omgeving, zouden fenotypes in de operationele COPD benadering de indeling van COPD patiënten in verschillende subgroepen mogelijk moeten maken om prognostische informatie te verschaffen, beter de juiste therapie te bepalen en klinisch relevante uitkomsten te beïnvloeden. Dit klinisch perspectief op zogenaamde fenotypes kan geïllustreerd worden aan de hand van de introductie van fenotypes zoals 'patiënten met frequente exacerbaties' of 'patiënten met bovenkwabemfyseem'. Verder worden betekenisvolle uitkomsten beperkt tot symptomen, exacerbaties, mate van ziekteprogressie of overlijden. Pulmonale en extra-pulmonale componenten van COPD worden zelfs gedefinieerd als ziektekenmerken en het willekeurig aanpakken van deze kenmerken als precisiegeneeskunde.

Het hoofddoel van dit proefschrift was om, op basis van pulmonale en extra-pulmonale kenmerken die tijdens een beginassessment werden bepaald bij patiënten met COPD verwezen voor longrevalidatie, clusters te identificeren en in deze clusters de differentiële uitkomsten te beoordelen om alzo de behandelinterventies beter te structureren. Deze samenvatting beoogt onze resultaten in perspectief te plaatsen. 


\section{Naar een controlepaneel voor COPD?}

In een poging de toekomst van de geneeskunde en de gezondheidszorg te beschrijven en intrinsiek gemotiveerd om abnormaal functioneren van het lichaam of lichaamscompartimenten te corrigeren, zijn binnen meervoudige contexten concepten voorgesteld als "precisiegeneeskunde", "gestratificeerde geneeskunde", "systeemgeneeskunde", "P4 geneeskunde" en "gepersonaliseerde geneeskunde". Hoewel alle termen verwijzen naar het groeperen van patiënten op basis van ziekterisico of respons op therapie met behulp van diagnostische tests of technieken, worden deze begrippen vaak door elkaar gebruikt. Dit is ook terug te vinden in de COPD-literatuur. Om de beperkingen van gestratificeerde geneeskunde (gebaseerd op het fenotype) te benadrukken en om vooruitgang te boeken in de gepersonaliseerde geneeskunde werd een COPD-controlepaneel voorgesteld met de domeinen "ernst", "activiteit" en "impact" van de ziekte COPD. Elk van deze domeinen moet informatie geven over de verschillende aspecten van de ziekte met betrekking tot prognostische waarde en/of met specifieke therapeutische eisen. Zoals piloten een controlepaneel gebruiken om veilig met een vliegtuig te vliegen, zo moet een COPD-controlepaneel het mogelijk maken de status van de relevante domeinen van de ziekte te visualiseren zodat artsen, die patiënten met COPD behandelen, de juiste therapeutische beslissingen kunnen nemen. Van patiënten wordt verwacht dat zij zich gedragen als een vliegtuig en gepersonaliseerd wordt gezien als het integreren van informatie afkomstig van deze verschillende bronnen. Echter, in deze benadering verzuimt de piloot de betrouwbaarheid van het bedieningspaneel te controleren om veilig te kunnen vliegen, de stimulus-respons variabiliteit van de verschillende meters op het dashboard te beoordelen en de bestemming van de passagiers te checken. Later omarmde de "respiratoire community" een "label-free" precisiegeneeskunde strategie voor de behandeling van patiënten met luchtwegaandoeningen, een strategie die gebaseerd is op de identificatie van "behandelbare kenmerken" bij elke patiënt. Deze strategie is geïntroduceerd als een nieuw paradigma voor het behandelen van complexe luchtwegaandoeningen, waarbij gepersonaliseerde geneeskunde inhoudt dat bij elk individu een verbetering van uitkomsten wordt bereikt.

In feite vormt deze geïndividualiseerde benadering al vele jaren de hoeksteen van de huidige praktijk van longrevalidatie (hoofdstuk 3). Longrevalidatie start namelijk met een multidimensioneel assessment bestaande uit de identificatie van pulmonale behandelbare kenmerken, extra-pulmonale functionele/gedragsmatige behandelbare kenmerken en gezondheidsstatus om op basis hiervan de behandeling te individualiseren naar de behoeften van de patiënt met complex COPD (hoofdstuk 3). In dit proefschrift laten wij duidelijk zien dat noch clustering op basis van uitgebreide longfunctie metingen (hoofdstuk 4), noch clustering op basis van pulmonale of extrapulmonale kenmerken en gezondheidsstatus (hoofdstuk 7) tot duidelijk te 
onderscheiden subgroepen van patiënten heeft geleid. Onze bevindingen in hoofdstuk 4 laten zien dat eenvoudige classificatie van COPD op basis van spirometrie de heterogeniteit van respiratoire stoornissen bij de doorverwezen patiënten onderschat. Op basis van spirometrie na inhalatie van een bronchodilator, diffusiecapaciteit van de long voor koolmonoxide, bodyplethysmografie, monddrukken, en arteriële bloedgassen, was het mogelijk om zeven longfunctieclusters te onderscheiden: patiënten met 1) een milde tot matige luchtwegobstructie en met een lichte diffusiestoornis, 2) matige tot ernstige luchtwegobstructie met een matige diffusiestoornis en ademhalingsspierzwakte, 3) matige tot ernstige luchtwegobstructie met een matige diffusiestoornis, 4) matige tot ernstige luchtwegobstructie met een lichte diffusiestoornis, 5) ernstige luchtwegobstructie met ernstige statische hyperinflatie en matige diffusiestoornis en ademhalingsspierzwakte, 6) ernstige luchtwegobstructie met ernstige statische hyperinflatie en matige diffusie-stoornis en 7) ernstige luchtwegobstructie met ernstige statische hyperinflatie en ernstige diffusiestoornis met ademhalingsspierzwakte en alveolaire hypoventilatie (hoofdstuk 4). De enorme overlap tussen deze clusters beperkt de voorspelbaarheid als het gaat om de individuele patiënt. Hoewel een uitgebreide longfunctie deel moet uitmaken van een beginassessment om de pathofysiologische ademhalingsstoornissen te begrijpen, toont hoofdstuk 4 ook aan dat zelfs een uitgebreid assessment en clustering van meetbare longfunctievariabelen de functionele prestaties en gezondheidsstatus bij deze patiënten slecht voorspellen. Hoofdstuk 7 geeft een samenvatting van de resultaten van clustering op basis van respiratoire, functionele en emotionele kenmerken. Zeven patiënten-profielen worden onderscheiden: 1) 'het algemeen best functionerende' cluster, 2) het 'ADL (activiteiten in het dagelijks leven) beperkte' cluster, 3) 'het multimorbide' cluster, 4) het cluster met 'een lagere ziektelast', 5 ) het 'emotioneel disfunctionerende' cluster, 6) het 'algemeen slechtst functionerende' cluster en 7) het 'fysiek disfunctionerende' cluster. Opnieuw wordt geconcludeerd dat deze clusters een doel- en persoonsgerichte aanpak niet kunnen vervangen (hoofdstuk 7).

Onze gegevens illustreren duidelijk dat zelfs het meest complexe "controlepaneel" zoals beschreven in hoofdstuk 7 niet bijdraagt aan de identificatie van bruikbare subgroepen van COPD patiënten die verwezen worden voor longrevalidatie. De gegevens beschreven in hoofdstuk 7 onderstrepen nogmaals dat de huidige aanbevelingen met betrekking tot het beoordelen van en het graderen van de ziekte COPD op basis van spirometrie, dyspneu, en/of gezondheidsstatus en exacerbaties en/of ziekenhuisopnames in de laatste 12 maanden de mogelijke behoeften bij deze fragiele, chronische patiënten ruim onderschatten. 


\section{Naar individuele doelen en waarden?}

Zoals beschreven in hoofdstuk 1, formuleerde de ACCP (American College of Chest Physicians) en ATS (American Thoracic Society) voor het eerst het begrip longrevalidatie als een programma dat moet trachten de individuele patiënt terug te brengen naar de hoogst mogelijke capaciteit die de longaandoening en de algehele levenssituatie toelaten oftewel het individu te herstellen naar het hoogst voor de patiënt bereikbare medische, mentale, emotionele, sociale en beroepsmatige potentieel. Anderen bevestigden dat longrevalidatie individueel op maat moet worden gemaakt en ontworpen is om het fysiek en sociaal functioneren en autonomie te optimaliseren. Vanuit dit perspectief moeten uitkomsten van longrevalidatie worden gedefinieerd als veranderingen in de somatische, fysieke, psychologische en sociale conditie die gunstige of ongunstige effecten op het welzijn van de patiënt weerspiegelen. Echter en waarschijnlijk gedreven door scepticisme over longrevalidatie, beperkte de ERS (European Respiratory Society) de uitkomsten tot klinisch en fysiologisch relevante uitkomstmaten. Later werd gepleit voor verbetering van uitkomsten met betrekking tot de fysieke en psychische conditie van personen met een chronische ademhalingsziekte.

In de praktijk zijn de uitkomsten van longrevalidatie grotendeels beperkt tot vermindering van symptomen, verbetering van de loopafstand en de gezondheidsstatus. Volgens de laatste GOLD (Global Initiative for Chronic Obstructive Lung Disease) richtlijnen zijn patiënten met een hoge symptoomlast en risico op exacerbaties geïndiceerd voor longrevalidatie. Echter, gegeven het profiel van een patiënt met COPD die wordt doorverwezen voor longrevalidatie, moeten uitkomstmaten verder gaan dan het meten van een standaard gezondheidsgerelateerde kwaliteit van leven en/of inspanningscapaciteit. Uitkomsten die er echt toe doen voor patiënten met complexe meervoudige problemen houden rekening met het ziek zijn van de patiënt, hoe het voor een persoon voelt om ziek te zijn of hoe iemand het verlies van zijn/haar gezondheid (ziekte) ervaart. Daarnaast moeten uitkomsten als het vermogen tot aanpassing en zelfmanagement ten aanzien van de sociale, fysieke en emotionele uitdagingen van hun leven worden overwogen.

Het effect van een multidimensionele uitkomstmaat, bestaande uit acht uitkomstmaten, is samengevat in hoofdstuk 5: uitkomsten van longrevalidatie bleven niet beperkt tot functionele prestaties ( 6 minuten loopafstand, fietsduurtijd of symptomen van dyspneu en gezondheidsstatus), ook angst en depressie, alsmede het uitvoeren en de tevredenheid over het uitvoeren van de problematische activiteiten in het dagelijks leven werden gemeten. Patiënten werden geclusterd in vier groepen met verschillende multidimensionele responsprofielen: zeer goede, goede, matige en slechte responders (hoofdstuk 5). Een belangrijke vaststelling is dat, in tegenstelling tot het huidige "evidence-based" denken, patiënten beslist baat hebben bij longrevalidatie: in het 
cluster met de zeer goede responders werd in $85 \%$ van de uitkomsten eenmaal en in 67 $\%$ van de uitkomsten tweemaal een minimaal klinisch relevante verbetering bereikt. Zelfs in het cluster met de slechte responders wordt in $11 \%$ van de multidimensionele uitkomsten klinisch relevante verbeteringen geïdentificeerd. In deze groep worden in het bijzonder patiënten gezien met een significant hogere tevredenheid voor de problematische activiteiten in het dagelijks leven, hetgeen duidt op een vergroting van het veerkrachtpotentieel na longrevalidatie. De vergelijking van de initiële patiëntenkenmerken tussen de vier verschillende responsprofielen in hoofdstuk 5 laat zien dat op basis van alleen fysiologische parameters geen onderscheid gemaakt kan worden tussen de vier clusters: patiënten in de groep met de zeer goede responders lijken zelfs de hoogste ziektelast te hebben. Hoofdstuk 6 bevestigt de beperkingen van longfunctietesten om uitkomsten van longrevalidatie te voorspellen: er wordt geen relatie gevonden tussen de zeven geïdentificeerde op longfunctie gebaseerde clusters en respons op longrevalidatie. De voorspelbaarheid verbetert ook niet op basis van een multidimensioneel assessment (hoofdstuk 8): deze studie bevestigt dat in de groep van de zeer goede responders de hoogste ziektelast wordt gevonden. Dit blijkt uit lage inspanningsprestaties en slechtere scores voor activiteiten in het dagelijks leven, angst, depressie en kwaliteit van leven, ondanks een betere longfunctie. De studie in hoofdstuk 8 bevestigt ook de bevindingen van hoofdstuk 5, namelijk dat het aantal zeer goede en goede responders opmerkelijk hoger is na een klinisch longrevalidatieprogramma. Deze bevindingen benadrukken niet alleen de noodzaak van integratie van psychosociale factoren en gedragsverandering in de behandeling voor mensen met chronische ademhalingsziekten, maar ook de uitdaging met betrekking tot het identificeren van de juiste patiënt voor de juiste uitkomst op het juiste moment.

In het laatste decennium is er meer aandacht gekomen voor "value-based health care" (d.w.z. gericht op het maximaliseren van de waarde van de zorg voor patiënten). Waarde wordt gedefinieerd als de bereikte gezondheidsresultaten gedeeld door de bestede kosten. In zijn baanbrekende publicatie stelde Michael Porter dat waarde altijd rond de klant moet worden gedefinieerd en dat het creëren van waarde de vergoeding voor alle actoren in het gezondheidszorgsysteem zou moeten bepalen. De uitkomsten in de teller moeten inherent aandoeningsspecifiek en multidimensioneel zijn. Het meten, rapporteren en vergelijken van uitkomsten zijn daarom cruciale stappen. Onze data met betrekking tot uitkomsten illustreren duidelijk de noodzaak van een multidimensionele uitkomstenevaluatie bij longrevalidatie. Opvallend is dat een interventiestrategie die zich beroept op individualisering op basis van de beoordeling van "individual needs" nog steeds behandeleffecten omarmt die grotendeels zijn afgeleid van farmacologische klinische trials. Het ontbreken van een multidimensionele uitkomstmaat of het vertrouwen op een beperkte set van gemiddelde behandeleffecten belemmert de inschatting van de 
werkelijke waarde van longrevalidatie. Een dergelijk gebrek aan prestatiemaatstaven belemmert ook het benchmarken van verschillende programma's of longrevalidatiesettings. Onduidelijkheid in relatie tot doelstellingen zullen een bedreiging vormen en het programma ondermijnen met als gevolg pseudobesparingen die onder de noemer 'kostenbesparingen' vallen. 


Impact section 



\section{Impact section}

This section describes the scientific and social impact of the conclusions of this PhDthesis. Moreover, target groups and the way that these target groups can be involved and insights of this thesis can be used are discussed.

\section{Main aim and conclusions of this thesis}

Understanding the complexity of chronic diseases, such as chronic obstructive pulmonary disease (COPD), and the adaptation of the health care system to implement new management strategies addressing the patients' needs, form still major challenges. In this PhD thesis, pulmonary rehabilitation is described as an integrated, comprehensive approach for patients with COPD and considered as an example of personalized, individualized care to patients with high needs and high disease burden. The patient tailored program, as described in this PhD thesis, forms the fundaments for standardization of pulmonary rehabilitation between the Dutch Lung Centers: each program starts with an integrated assessment in order to identify existing needs and to individualize treatment and ends with a systematic outcome evaluation. At Ciro, a specialized center for integrated rehabilitation, these data of the initial assessment and outcome were gathered structurally and anonymously to possibly identify drivers of disease burden and to support personalized health care decision-making. Clustering and visualization techniques, by self-organizing maps, were used to create an ordered representation of selected attributes: patients have been ordered by their overall similarity concerning their clinically relevant attributes. In five chapters of this thesis, the focus was on identification of traits-based clusters in order to reduce variation of interventions, maximize outcomes and as a consequence improve cost-effectiveness of pulmonary rehabilitation and to find and optimize clinical pathways in a pulmonary rehabilitation setting. The main conclusion was that neither clustering based on comprehensive lung function measurements nor clustering based on respiratory, functional and emotional traits and health status has led to recognizable clinical pathways.

The first clustering, that was based on comprehensive lung function measurements, clearly demonstrated that a simple classification of COPD based on spirometry alone underestimates the heterogeneity in respiratory impairment. Although comprehensive lung function has to be part of an initial assessment to understand the pathophysiological respiratory impairments, clustering of measurable lung variables poorly predict functional performance and health status in these patients. When lung function attributes were extended with functional traits, emotional traits and health status, seven patient profiles could be discerned, but again, this clustering did not capture the individual burden of the patients referred for pulmonary rehabilitation and 
does not contribute to identification of actionable subgroups of COPD patients referred for pulmonary rehabilitation.

In this PhD thesis a multidimensional outcome measure existing of eight outcome measures was used to evaluate response to pulmonary rehabilitation. We demonstrated that improvements were found not only for functional performance, but also for anxiety and depression as well performance and satisfaction with this performance of problematic activities in daily life. Based on this multidimensional outcome measure four groups with distinct multidimensional response profiles could be found: very good, good, moderate and poor responders. Using the eight outcome measures in order to evaluate response to pulmonary rehabilitation for the seven lung function clusters, it was concluded that baseline lung function cannot be used to identify good responders to pulmonary rehabilitation, and therefore, cannot be used as the only criterion for referral to pulmonary rehabilitation in patients with COPD. Predictability of assessment is not improved by including more attributes. However, by applying a multidimensional outcome index, this study demonstrates that a high prerehabilitation disease burden, manifested by low exercise performance, worse scores on activities in daily living, anxiety, depression and quality of life despite better pulmonary traits does not exclude good response after intervention. The study also confirms that the number of very good and good responders is remarkably higher after an inpatient pulmonary rehabilitation program in comparison with outpatient programs. The findings in this thesis emphasize not only the need of integrating psychosocial factors and behavior change in the care of people with chronic respiratory diseases, but also the challenge to identify the right patient for the right treatment with the right outcome at the right time.

\section{Relevance}

Chronic respiratory diseases with a high prevalence, economical and societal burden of COPD, are among the most common non-communicable diseases. A small proportion of these patients with COPD experiences multidimensional complex problems as was demonstrated in this PhD thesis. These high-need patients, facing the fragmented health care system and frequently using the emergency department for their health care needs, are also identified as high-cost patients. Integrated or coordinated care, a worldwide trend in health care reforms and new organizational arrangements, may be seen as a response to the fragmented delivery of health services. Importantly, a clear distinction should be made between care for patients with high-multidimensionalneeds and usual care. Moreover, needs in these patients as well as in our cohort extend largely beyond care for physical or medical ailments to behavior and social aspects. This PhD thesis provides valuable insights in order to optimize health care services for patients with high-needs.

First, the results in this $\mathrm{PhD}$ thesis underscore the limitation of current recommendations to assess and grade COPD on spirometry, dyspnea, and/or health 
status and exacerbations and/or hospitalizations in the last 12 months. Indeed, such a limited assessment largely underestimate the number and heterogeneity of traits involved in the functional and emotional disability as experienced by these frail chronic patients. Attention should be drawn to this group of patients with comorbidities, limitations of activities in daily living, emotional and behavioral problems as they have to deal with a lack of coordination within the health care system. In order to recognize timely the group of patients with high-needs and treating them appropriately, and ideally reduce the cost of care, additional work will be needed to develop an taxonomy which fits with the heterogeneity of COPD. Our study hopes to especially creates awareness for the frail COPD patients.

Second, our data on outcomes clearly shows the relevance of a multidimensional outcome evaluation in pulmonary rehabilitation. Moreover, pulmonary rehabilitation delivered as an integrated and comprehensive intervention demonstrated very good response to treatment in these patients with multidimensional needs as was demonstrated in the previous chapters. In order to improve outcomes that really matter to patients with complex chronic diseases, chronic care models must be responsive to the identified needs. Current outcome measures as functional performance testing or health status measurement does not capture the comprehensive impact of a pulmonary rehabilitation program.

Third, considering the patient profiles and characteristics studied in this PhD thesis, personalized medicine as the concept of better understanding the complexity of COPD based on stratifying patients, might aspire more control over the optimization of medical treatment results, more precision, increasing predictability of disease, and thus control over the outcomes: a patient-centered care model that builds on the person's agency and empowerment remains important. A personalized management program has to put the patient in the center and clearly clarify the role of the patient. Patients should be able to discuss what's important in life, to specify their desirable way of living, their sense of life and their meaning of quality of life which should be fundamental for defining successful, individualized treatment goals meeting patients' expectations.

\section{Target groups and using new insights}

\section{Patients with high-needs}

The patient him- or herself who is not able to self-manage his/her chronic condition, who daily experience his or her unmet needs, will benefit from an integrated and comprehensive treatment program that is tailored to his or her individual needs. Based on such a program, this PhD thesis illustrated clinically relevant outcomes even in patients with the highest burden of the disease at baseline. 
Since patients with high-needs have more than one chronic condition, different patient advocacy organizations should work together in order to build awareness for this specific group.

\section{Health care professionals}

Since the growing reliance on evidence based medicine concepts in daily practice, health care professionals and organizations have been confronted with the difficulty to follow the current principles of value-based health care particularly for those patients referred for their high needs. Needs in all these patients as well as in our cohort extend largely beyond care for physical or medical ailments to behavioral and social aspects. Moreover, as already described above, health care professionals have to deal with the increased uncertainty of the outcome. In clinical practice, a patient-centered approach is needed fostering interactions in which clinicians and patients engage in two-way sharing of information; explore patients' values and preferences; help patients and their families make clinical decisions; and enable patients to follow through with often difficult behavioral changes needed to maintain or improve health.

\section{Health care organizations}

This premise of different levels and dimensions of a clinical problem implies not only the collaboration of different medical disciplines but also a holistic approach of patients beyond the fragmented vision of each specialty. Traditional leadership and management approaches are no longer sufficient to deal with the organizational and contextual complexity associated with delivering care for these high-needs patients. Indeed, health care organizations should transform to transdisciplinary organizations bringing together actors from varied backgrounds, promoting interdependence among them, and form dynamic collectives with common goals. Major emphasis should be made on inclusion of patients into decision-making to personalize care plans instead of delivering a standard treatment for every individual meeting certain criteria. Leadership in such organizations presumes enhancing interactive and adaptive dynamics and monitoring the organization to better understand the different forces influencing the emerging adaptive dynamics.

\section{Health care authorities and health insurers}

As one of the most expensive and challenging populations for the current health care system will remain underserved when care models deal only with physical ailments. Care models to be effective must also address the social and behavioral factors in play for a given patient. Improving care for the high-need patients finally aiming to reduce cost of delivering care, policy action should focus on addressing the existing constraints and complexities preventing the integration of medical, behavioral, and social services. Major emphasis should be made on inclusion of patients into decision-making to personalize care plans instead of delivering a standard treatment for every individual meeting certain criteria. 


\section{Research groups}

Although clustering in this thesis shed new light on the impairments in the different health domains in patients with COPD, personalized medicine based on identifiable phenotypes remains a challenge in the real life health care setting. An effort should be made on exploring the potential of data collected in daily clinical practice as a source of up-to-date minimally biased population-specific knowledge and on implementing this information into clinical practice in a more agile manner than only relying on randomized controlled trials. Furthermore, research in these patients cannot be restricted to one discipline, but has to be apply a transdisciplinary and holistic approach.

\section{Activities}

Pulmonary rehabilitation offers a model for a holistic approach of patients with COPD in particular and chronic illness conditions in general.

Besides the publication of different chapters, the results of this PhD thesis are helpful in ongoing discussions with health care authorities and health insurers. Additionally, given the limitations of the current clustering, it offers opportunities and insights to optimize the current organization of pulmonary rehabilitation by reconsidering measurements of the initial assessment, and by optimizing the transdisciplinary and learning organization and leadership. 

List of publications 



\section{List of publications}

Augustin IML, Spruit MA, Franssen FME, Gaffron S, van Merode F, Wouters EFM. Incorporating Comprehensive Assessment Parameters to Better Characterize and Plan Rehabilitation for Persons with Chronic Obstructive Pulmonary Disease. J Am Med Dir Assoc. 2020 Dec;21(12):1986-1991.e3.

Spruit MA, Van't Hul A, Vreeken HL, Beekman E, Post MHT, Meerhoff GA, Van der Valk AL, Zagers C, Sillen MJH, Vooijs M, Custers J, Muris J, Langer D, Donkers J, Bregman M, Tissink L, Bergkamp E, Wempe J, Houben-Wilke S, Augustin IML, Bij de Vaate E, Franssen FFM, Van Ranst D, Van der Vaart H, Antons J, Van Doormaal M, Koolen EH, Van der Wees P, Van Snippenburg R, Janssen DJA, Simons S. Profiling of Patients with COPD for Adequate Referral to Exercise-Based Care: The Dutch Model. Sports Med. 2020 Aug;50(8):1421-1429.

Augustin IML, Wouters EFM, Houben-Wilke S, Gaffron S, Janssen DJA, Franssen FME, Spruit MA. Comprehensive Lung Function Assessment Does not Allow to Infer Response to Pulmonary Rehabilitation in Patients with COPD. J Clin Med. 2018 Dec 27;8(1):27.

Stoilkova-Hartmann A, Franssen FME, Augustin IML, Wouters EFM, Barnard KD. COPD patient education and support - Achieving patient-centredness. Patient Educ Couns. 2018 Nov;101(11):2031-2036.

Augustin IML, Spruit MA, Houben-Wilke S, Franssen FME, Vanfleteren LEGW, Gaffron S, Janssen DJA, Wouters EFM. The respiratory physiome: Clustering based on a comprehensive lung function assessment in patients with COPD. PLoS One. 2018 Sep 12;13(9):e0201593.

Houben-Wilke S, Augustin IM, Vercoulen JH, van Ranst D, Bij de Vaate E, Wempe JB, Spruit MA, Wouters EFM, Franssen FME. COPD stands for complex obstructive pulmonary disease. Eur Respir Rev. 2018 Jun 6;27(148):180027.

Wouters EFM, Wouters BBREF, Augustin IML, Houben-Wilke S, Vanfleteren LEGW, Franssen FME. Personalised pulmonary rehabilitation in COPD. Eur Respir Rev. 2018 Mar 28;27(147):170125. 
Houben-Wilke S, Augustin IM, Wouters BB, Stevens RA, Janssen DJ, Spruit MA, Vanfleteren LE, Franssen FM, Wouters EF. The patient with a complex chronic respiratory disease: a specialist of his own life? Expert Rev Respir Med. 2017 Dec;11(12):919-924.

Wouters EF, Wouters BB, Augustin IM, Franssen FM. Personalized medicine and chronic obstructive pulmonary disease. Curr Opin Pulm Med. 2017 May;23(3):241-246.

Augustin IML and Wouters EFM. Process of Pulmonary Rehabilitation and Program Organization Mini Review: J Card Pulm Rehabil 2017, 1: 109.

Janssen DJ, Wilke S, Smid DE, Franssen FM, Augustin IM, Wouters EF, Spruit MA. Relationship between pulmonary rehabilitation and care dependency in COPD. Thorax. 2016 Nov;71(11):1054-1056.

Wouters EF, Augustin IM. COPD health-care delivery: a holistic and dynamic approach is needed. Lancet Respir Med. 2016 Jun;4(6):e30-1.

Spruit MA, Augustin IM, Vanfleteren LE, Janssen DJ, Gaffron S, Pennings HJ, Smeenk F, Pieters W, van den Bergh JJ, Michels AJ, Groenen MT, Rutten EP, Wouters EF, Franssen FM; CIRO+ Rehabilitation Network. Differential response to pulmonary rehabilitation in COPD: multidimensional profiling. Eur Respir J. 2015 Dec;46(6):1625-35.

Augustin IML, Spruit MA, Franssen FME, Wouters EFM. Understanding Complexity of Chronic Non-Communicable Diseases: An Integrated Approach for Personalized Management of Patients with COPD. Clin Res Pulmonol. 2015; 3(2): 1034.

Meis JJ, Bosma CB, Spruit MA, Franssen FM, Janssen DJ, Teixeira PJ, Augustin IM, Wouters EF, de Vries NK, Schols AM, Kremers SP. A qualitative assessment of COPD patients' experiences of pulmonary rehabilitation and guidance by healthcare professionals. Respir Med. 2014 Mar;108(3):500-10.

Wouters EF, Augustin IM. Process of pulmonary rehabilitation and program organization. Eur J Phys Rehabil Med. 2011 Sep;47(3):475-82.

Wouters EF, Vanderhoven IM. Pulmonary rehabilitation and integrated care. Semin Respir Crit Care Med. 2009 Dec;30(6):713-20.

Emiel FM Wouters \& Ingrid ML Vanderhoven-Augustin. Importance of integrated care for the chronic obstructive pulmonary disease patient. Therapy. 2009. 6: 831-835. 
Spruit MA, Vanderhoven-Augustin I, Janssen PP, Wouters EF. Integration of pulmonary rehabilitation in COPD. Lancet. 2008 Jan 5;371(9606):12-3.

Creutzberg EC, Wouters EF, Vanderhoven-Augustin IM, Dentener MA, Schols AM. Disturbances in leptin metabolism are related to energy imbalance during acute exacerbations of chronic obstructive pulmonary disease. Am J Respir Crit Care Med. 2000 Oct;162(4 Pt 1):1239-45.

Wesseling GJ, Vanderhoven-Augustin IM, Wouters EF. Forced oscillation technique and spirometry in cold air provocation tests. Thorax. 1993 Mar;48(3):254-9. 

Dankwoord 



\section{Dankwoord}

Dat de organisatie Ciro, eerst als onderwerp van mijn masterthesis, mij naar deze mijlpaal zou brengen, had ik nooit durven dromen. Dit moment, waaraan vele jaren vooraf gingen, voelt nu toch wel ongewoon. Het is door de steun van velen dat ik de eindbestemming heb gehaald. Met alle hindernissen onderweg leek de reis eindeloos te duren, maar ik heb veel geleerd en blik met dankbaarheid terug.

Allereerst wil ik mijn promotors Prof. dr. Emiel Wouters, Prof. dr. Frits van Merode en Prof. dr. Martijn Spruit bedanken.

Beste Miel, nog voor het afronden van mijn masterthesis, in 2005, vroeg je of een promotietraject iets voor mij zou zijn. Voor mij op dat moment ondenkbaar, maar onze samenwerking als raad van bestuur binnen de context van een kenniscentrum als Ciro zorgde ook voor een innerlijke onrust: de brug leggen tussen wetenschap en de primaire zorg hield in dat ik meer inzicht wilde verwerven. Ook al wist ik dat dit traject in combinatie met mijn rol als bestuurder en mijn gezin bepaald geen sinecure zou zijn, gestaag werkte ik aan deze volgende stap. Jouw onvermoeibare kritische en vooruitziende blik kon ik zeer waarderen, doch jouw 'rode pen' kon me ook moedeloos maken. Herhalen, herzien, andere invalshoeken kiezen, vallen en opstaan, ik heb de zoektocht als leidraad ervaren. Ik was zeker niet de gemakkelijkste promovendus en weet dat ik erg eigenwijs kon en kan zijn, maar desondanks wist je me mee te nemen naar waardevolle andere en nieuwe perspectieven. Dank voor jouw enorme geduld en voor het vertrouwen dat ik mocht ervaren.

Beste Frits, begin januari 2018 hadden wij een eerste overleg met als onderwerp "promoveren en Value Based Health Care". Met belangstelling nam je de eerste publicaties door: je wilde graag een extra dimensie aan het onderzoek toevoegen. Balancerend tussen inhoud en organisatie, bracht je evenwicht in het onderzoek. Het blijft immers een uitdaging om deze twee werelden bij elkaar te brengen. Jouw andere blik op de clusteranalyses, maar vooral ook de toevoeging van organisatorische aspecten in het laatste hoofdstuk, het waren de laatste loodjes, maar het bracht uiteindelijk zeer interessante inzichten en heeft mijn nieuwsgierigheid voor de toekomst zeker aangewakkerd. Dank voor jouw begeleiding en het vertrouwen in dit traject.

Beste Martijn, 1 september 2005 begonnen we gezamenlijk in Horn. Jij met het voortzetten van jouw passie voor onderzoek en ik aan de slag met de herinrichting van processen. We bleven elkaar tegenkomen en toegegeven, jouw enthousiasme voor data, voor het onderzoek, de nieuwe inzichten, het werkte aanstekelijk. Aanvankelijk stelde jij vragen bij mijn ambitie en uiteraard heb ook ik vaak gedacht "Waar ben ik aan begonnen?". Het toch aandurven mij te begeleiden, ik kon het zeer waarderen. Ik heb veel geleerd van jouw expertise, je maakte me vooral wegwijs op het gebied van de 
methodologie en de statistische analyses. En intussen blijven we elkaar tegenkomen als collegae-bestuurders en worden discussies over inhoud en organisatie voortgezet. Dank dat je mee richting hebt gegeven aan dit traject.

Aan de aandeelhouders, Maastricht Universitair Medisch Centrum+ (MUMC+) en Stichting Proteion, en de raad van commissarissen van $\mathrm{CIRO}+\mathrm{BV}$, dank voor jullie steun en interesse gedurende dit traject.

Beste Frits (Prof. dr. Franssen), als collega-bestuurder loop ik nu vaker even de hoek om, ook omdat ik dan toch weer een inhoudelijke vraag heb. Dank dat je me als medisch specialist weer even verder helpt in het begrijpen. En zoals afgesproken, eerst mijn promotie en dan jouw inaugurale rede.

Daisy, het mag nu zwart op wit: prof. dr. D. Janssen. Jij die 'zorg op maat' en 'samen beslissen' hoog in het vaandel heeft. Het is mogelijk een vanzelfsprekendheid, maar jij slaagt erin jouw kennis in de praktijk te brengen en gaat zichtbaar voor de individualisering van zorg. Dank voor jouw inzichten en ondersteuning. En uiteraard dank aan de gehele medische raad voor het vertrouwen in mij en de toekomst van Ciro.

Aan alle co-auteurs, het Ciro-netwerk, dank voor jullie feedback en bijdrage aan dit manuscript. Sarah, dank voor het werk wat voorafging aan deze thesis. Jij hebt samen met het Longfonds en GlaxoSmithKline de Chance Studie mogelijk gemaakt.

Swetlana, thank you for your help with all kind of statistical questions at the most inconvenient moments. I would also like to thank your colleague Simon for the individual course and dr. Gerhard Kranner, thank you for the warm welcome in Vienna.

Miriam, als ik dan nog vragen had over statistiek of twijfelde aan getallen, jouw bereidwilligheid om te helpen kent geen grenzen. Het bewaken van data, het bewaken van privacy, er is niemand die deze rol beter kan vervullen.

De cliëntenraad als vertegenwoordiger van alle patiënten ben ik zeer erkentelijk. Jullie geven mij interessante inzichten en zorgen voor verrassende invalshoeken. Dank voor jullie grote betrokkenheid.

Rosita, regelmatig sparren we over kwaliteit van zorg, over het standaardiseren van processen en de valkuilen: ook voor jou zeker geen 'One size fits all', maar vooral oog blijven houden voor de inhoud en de onvoorspelbaarheid. Gedurende dit promotietraject mocht ik een enorme steun van jou ervaren. Dank voor alles.

Maurice, al zijn jouw dienstjaren bij Ciro relatief kort en ook al heb jij een financiële achtergrond, ik waardeer jouw reflectie op inhoud. Meer nog, ook voor jou is de inhoud leidend. 
Ellen, ik realiseer me dat het niet evident is iemand als mij te ondersteunen. Toch wist jij steeds de rust te bewaren wanneer mijn agenda weer te chaotisch dreigde te worden. Dank voor jouw hulp en het zorgen van tegenwicht.

Gerrie, al heb jij binnen Ciro een andere rol, onze samenwerking is zeker niet minder. Dank voor jouw blijvende belangstelling tijdens dit traject en jouw bekommernissen.

Paula, Jozé, Gwen, Maurice, Jérôme, Marco, Sarah, Stephanie, Daisy, Nicole C, Manu, Sjef, Sandra en Nicole S, onze inhoudelijke discussies worden steeds boeiender. Dank voor jullie engagement en voor de openheid, het inzicht geven in de praktijk.

Eveline, Yoni, Monique, Glenn, Marjanka, Joost, Koen, Lilian, Annemiek, ik waardeer jullie support en belangstelling, dank voor het ontzorgen.

De ondernemingsraad, dank voor jullie zorgvuldigheid en voortvarendheid in het belang van de medewerker èn het belang van de patiënt. Uiteraard dank aan alle medewerkers van Ciro. Mogelijk was niet bij iedereen bekend dat ik op de achtergrond werkte aan dit traject, toch mocht ik een indirecte motivatie ervaren. Jullie bevlogenheid, ik beschouw het als een voorrecht om samen met jullie te leren en 'patiënt als specialist van zijn eigen leven' in de praktijk te verwezenlijken.

De leden van de beoordelingscommissie, Prof. dr. Schols als voorzitter, Prof. dr. van den Beuken-van Everdingen, Prof. dr. Ruwaard, Prof. dr. Jacobs (Tilburg University) en Prof. dr. Kenn (University of Marburg - Germany) wil ik bedanken voor hun grondige beoordeling en goedkeuring van dit manuscript. Thank you, Prof. dr. Kenn for your thorough assessment and approval of this dissertation.

Mijn ouders, (schoon)familie en vrienden, wat ik allemaal achter de coulissen deed was niet altijd te begrijpen en het begrip 'tijd' leek gaandeweg een steeds engere betekenis te krijgen. Dank voor al dat wachten.

Patricia, ons wekelijks rondje joggen in het bos is absoluut belangrijk voor ons fysiek functioneren. Zeker niet minder zijn onze gesprekjes onderweg. Mogelijk onderschat je het, maar je bent een enorme steun voor me. Dank!

Rita, vertoevend in een context waar dit traject vooral op de achtergrond bleef, wil ik je bedanken voor vele waardevolle momenten. Je deed me beseffen dat er ook nog een andere wereld bestaat. 
(Tante) Jé, een speciaal woord van dank wil ik aan jou richten. Jouw aandacht, jouw zorg, jouw steun, vooral in moeilijke tijden, is zo waardevol. Steeds wist je me met jouw wijze woorden, jouw levenservaring weer dat extra duwtje in de rug te geven.

Lieve Johan, lieve broer, hoezeer heeft onze context onze wegen richting gegeven en hoe trots konden we op elkaar zijn. Dit moment had ik zo graag met jou gedeeld. Johan, de wijze waarop jij samen met jouw gezin, Karen, Zeno en Matisse grenzen verlegde, dit is niet in woorden uit te drukken. Prognoses, je behoorde allerminst tot het gemiddelde. Wat hebben wij hierdoor nog vele mooie momenten samen mogen beleven. Karen, ik wil je voor heel veel bedanken. Zeno en Matisse, het is een herhaling van woorden, maar als zus van jullie papa zal ik zijn trots blijvend uitdragen. Jullie reactie op de uitkomsten van de beoordelingscommissie: wat deed dat goed!

Koen en Jorrit, jullie als paranimfen, wat ben ik trots op jullie. Ik kijk vol bewondering naar de weg die jullie afleggen. Ik weet niet zo goed hoe jullie te bedanken, een moeder die onconventionele paden bewandelde: als ik twijfelde of ik wel het juiste deed, dan wisten jullie me steeds weer te overtuigen. Wat heb ik me steeds gesteund gevoeld door jullie!

Kathleen, dank dat jij er bent! Jij en Koen samen maken de reis erg bijzonder. Eind 2020 wordt jullie zoon en onze kleinzoon geboren. Groots is zijn impact!

Jorrit, jij weet ons steeds weer opnieuw te boeien door ons mee te nemen in jouw wereld van creativiteit. Dank voor de ontmoeting met Stefan Serneels en het maken van de cover!

Pascal, ik weet dat jij niet van dankwoorden houdt, maar sta me toch toe. Elkaar de ruimte geven, al valt te twijfelen over een evenwichtige verdeling, luidt ons levensmotto. Jij bent er steeds geweest voor onze zonen en zorgde dat ik 'mijn handen vrij' had voor mijn werk. Je wist me, zeker door jouw passie voor kunst, steeds weer opnieuw te doen geloven in dit traject. Wat had ik graag jouw werk op de cover, maar we hebben samen een andere keuze gemaakt. Driedimensionaliteit laat zich immers moeilijk in twee dimensies weergeven. Hiervoor is wisselen van perspectief nodig.

Lieve Louis, jouw vrolijkheid en energie, jij hebt ons terug de tijd gegeven. 


Curriculum Vitae 



\section{Curriculum Vitae}

Ingrid Augustin werd geboren op 7 juli 1963 in Heerlen. Na het afronden van het Hoger Secundair Onderwijs, onderverdeling Handel, aan het Sint-Jozefinstituut te Hoeselt (België) in 1981, begon ze in het toenmalig ziekenhuis Sint Annadal in Maastricht met de driejarige opleiding tot longfunctielaborant. Ze combineerde werken en leren en behaalde dit diploma in 1984. Haar carrière als leidinggevende startte in 1991. Na verscheidene jaren werkzaam te zijn geweest als hoofd van de afdeling longfunctie, voltooide ze in 2002 de tweejarige Opleiding tot Kader in de Gezondheidszorg aan de Hogeschool Zuyd Heerlen. In hetzelfde jaar aanvaardde ze de functie als manager van de afdeling longfunctie, de verpleegafdeling en polikliniek longziekten van het Academisch Ziekenhuis Maastricht. In 2003 startte ze met de masteropleiding Beleid en Beheer van de Gezondheidszorg bij de Faculteit Gezondheidswetenschappen aan de Universiteit Maastricht. In 2005 vervolgde ze haar carrière als algemeen manager, een jaar later als directeur, van Ciro (kennis- en behandelcentrum voor patiënten met een complex chronische longaandoening, chronisch hartfalen en/of slaapgerelateerde ademhalingsstoornissen), toen nog onderdeel van Stichting Proteion. Haar doctoraaldiploma behaalde ze in 2006. Het onderwerp van haar doctoraalscriptie "Besturen in een zorgketen, een ontwerpgericht onderzoek naar het vergroten van bestuurbaarheid van een organisatie in een zorgketen" vormde de basis voor het herinrichten van de processen in Ciro. De horizontale organisatiestructuur heeft geleid tot het structureel verzamelen van gegevens zodat het verkrijgen van nieuwe inzichten en de evaluatie van de uitkomsten van de geleverde zorg mogelijk werden gemaakt. Tegelijkertijd werd een grotere wendbaarheid van de organisatie en een hogere efficiency bewerkstelligd. Bij de oprichting van CIRO+ BV door de aandeelhouders Maastricht Universitair Medisch Centrum+ (MUMC+) en Stichting Proteion in 2010, werd ze benoemd tot lid raad van bestuur. Haar interesse voor inhoud en haar overtuiging dat het inrichten van zorg begint bij het begrijpen van de patiënt, vormde de aanleiding om onder supervisie van Prof. dr. Wouters, Prof. dr. van Merode en Prof. dr. Spruit haar ambities een vervolg te geven middels een PhD traject. Ingrid Augustin is momenteel voorzitter van de raad van bestuur van $\mathrm{CIRO}+\mathrm{BV}$. 